Supporting Information

\title{
Cross-Dimerization of 2,5-Dihydrofuran with Conjugated Dienes Catalyzed by (Chiral Diene)ruthenium(0) Complexes and Origins of the Enantioselectivity
}

Masafumi Hirano, ${ }^{* 1}$ (B) Shuhei Machida, ${ }^{1}$ Ryota Abe, ${ }^{1}$ Takuya Mishina, ${ }^{1}$ Nobuyuki Komine, ${ }^{1}$

(D) Hsyueh-Liang $\mathrm{Wu}^{2}$

${ }^{1}$ Department of Applied Chemistry, Graduate School of Engineering, Tokyo University of Agriculture and Technology, 2-24-16 Nakacho, Koganei, Tokyo 184-8588, Japan

${ }^{2}$ Department of Chemistry, National Taiwan Normal University, No.88, Section 4, Tingzhou Road, Taipei, 11677, Taiwan

Contact email information: hrc@cc.tuat.ac.jp (MH) 


\section{Contents:}

\section{Spectroscopic Charts}

(1S,4S)-1,7,7-Trimethyl-2,5-bis(2-methylphenyl)bicylo[2.2.1]hepta-2,5-diene [(1S,4S)-(2-Tol)2-bhd*] S5 (1S,4S)-1,7,7-Trimethyl-2,5-bis(3-methylphenyl)bicylo[2.2.1]hepta-2,5-diene [(1S,4S)-(3-Tol) $)_{2}$-bhd*] S6 (1S,4R,8R)-5-Methoxy-1,5-dimethyl-2-(1-naphtyl)bicyclo[2.2.2]octa-2,5-diene [(1S,4R,8R)-(1-Nap)-bod*]

$\left[\mathrm{Ru}(\mathrm{acac})_{2}\left\{\eta^{4}-(1 S, 4 S)-\mathrm{Ph}_{2}-\mathrm{bhd}^{*}\right\}\right](\mathbf{1 a})$

$\left[\mathrm{Ru}(\mathrm{acac})_{2}\left\{\eta^{4}-(1 \mathrm{~S}, 4 S)-(1-\mathrm{Nap})_{2}-\mathrm{bhd}^{*}\right\}\right](\mathbf{1} \mathbf{b})$

$\left[\mathrm{Ru}(\mathrm{acac})_{2}\left\{\eta^{4}-(1 \mathrm{~S}, 4 \mathrm{~S})-(2-\mathrm{Nap})_{2}-\mathrm{bhd}^{*}\right\}\right](\mathbf{1 c}) \quad \mathrm{S} 14$

$\left[\mathrm{Ru}(\mathrm{acac})_{2}\left\{\eta^{4}-(1 \mathrm{~S}, 4 S)-\left(2-\mathrm{Tol}_{2}\right)_{2}-\mathrm{bhd}^{*}\right\}\right](\mathbf{1 d}) \quad$ S16

$\left[\mathrm{Ru}(\mathrm{acac})_{2}\left\{\eta^{4}-(1 \mathrm{~S}, 4 \mathrm{~S})-(3-\mathrm{Tol})_{2}-\mathrm{bhd}^{*}\right](\mathbf{1 e}) \quad \mathrm{S} 18\right.$

$\left[\mathrm{Ru}(\mathrm{acac})_{2}\left\{\eta^{4}-(1 S, 4 S)-\mathrm{Ph}_{2}-\mathrm{bod}^{*}\right\}\right](\mathbf{1 f}) \quad \mathrm{S} 19$

$\left[\mathrm{Ru}(\mathrm{acac})_{2}\left\{\eta^{4}-(1 \mathrm{~S}, 4 R, 8 R)-(1-\mathrm{Nap})-\mathrm{bod}^{*}\right\}\right](\mathbf{1 h}) \quad \mathrm{S} 21$

$\left[\mathrm{Ru}\left(\eta^{6}\right.\right.$-naphthalene) $\left.\left\{\eta^{4}-(1 \mathrm{~S}, 4 S)-\mathrm{Ph}_{2}-\mathrm{bhd}^{*}\right\}\right](\mathbf{2 a}) \quad$ S22

$\left[\mathrm{Ru}\left(\eta^{6}\right.\right.$-naphthalene) $\left\{\eta^{4}-(1 \mathrm{~S}, 4 S)-(3-\mathrm{Tol})_{2}\right.$-bhd $\left.\left.{ }^{*}\right\}\right](\mathbf{2 e}) \quad$ S23

$\left[\mathrm{Ru}\left(\eta^{6}\right.\right.$-naphthalene) $\left\{\eta^{4}-(1 \mathrm{~S}, 4 S)-\mathrm{Ph}_{2}\right.$-bod $\left.\left.{ }^{*}\right\}\right](\mathbf{2 f}) \quad \mathrm{S} 25$

$\left[\mathrm{Ru}\left(\eta^{6}\right.\right.$-naphthalene) $\left\{\eta^{4}-(1 \mathrm{~S}, 4 R, 8 R)-(1-\mathrm{Nap})-\right.$ bod $\left.\left.^{*}\right\}\right](\mathbf{2 h}) \quad \mathrm{S} 26$

3-(2,3-dimethylbut-2-en-1-yl)-2,3-dihydrofuran (6aa) $\quad$ S27

(Z)-3-(pen-2-en-1-yl)-2,3-dihydrofuran (6ab) and (Z)-3-(pen-2-en-1-yl)-2,3-dihydrofuran (9ab) S28 3-(3-methylbut-2-en-1-yl)-2,3-dihydrofuran (6ac) and 3-(2-methylbut-2-en-1-yl)-2,3-dihydrofuran (9ac)

S28

3-(3,7-dimethylocta-2,6-diene-1-yl)-2,3-dihydrofuran (6ad) and 3-(2-ethylidene-6-methylhept-5-en-1-

yl)-2,3-dihydrofuran (9ad) S30

3-(2,3-dimethylpent-2-en-1-yl)-2,3-dihydrofuran (6ae) and 3-(3,4-dimethylpent-3-en-2-yl)-2,3-

dihydrofuran (9ae)

S32

(E)-3-(3-phenylbut-2-en-1-yl)-2,3-dihydrofuran (6af) and (E)-3-(2-phenylbut-2-en-1-yl)-2,3-dihydrofuran (9af)

(E)-3-(3,4-diphenylbut-2-en-1-yl)-2,3-dihydrofuran (6ag) S35

(Z)-3-(3-methyl-4-phenylbut-2-en-1-yl)-2,3-dihydrofuran (6ah) and (Z)-3-(2-methyl-1-phenylbut-2-en-1yl)-2,3-dihydrofuran (9ah) S37

(Z)-3-(2-methyl-4-phenylbut-2-en-1-yl)-2,3-dihydrofuran (6ai) S38

(E)-3-(3-2-naphthylbut-2-en-1-yl)-2,3-dihydrofuran (6aj) and (E)-3-(2-2-naphthylbut-2-en-1-yl)-2,3dihydrofuran (9aj) 
3-(2,3-dimethylbut-2,3-en-1-yl)-2,3-dihydrothiophene 1,1-dioxide (6ba) S40

3-(2,3-dimethylbut-2,3-en-1-yl)-1-tosyl-2,3-dihydro-1H-pyrrole (6ca) S42

4-(2.3-dimethylbut-2-en-1-yl)-1-propylpyrrolidin-2-ol (6da) S43

4-(2,3-dimethylbu-2-en-1-yl)dihydrofuran-2(3H)-one (7aa) S44

4-(3-methylbut-2-en-1-yl)dihydrofuran-2(3H)-one (7ac) and 4-(2-methylbut-2-en-1-yl)dihydrofuran-

2(3H)-one (7ac') S46

4-(3-phenylbut-2-en-1-yl)dihydrofuran-2(3H)-one (7af) S47

4-(2-phenylbut-2-en-1-yl)dihydrofuran-2(3H)-one (7af') S50

4-(3-2-naphthylbut-2-en-1-yl)dihydrofuran-2(3H)-one (7aj) S52

4-(2-2-naphthylbut-2-en-1-yl)dihydrofuran-2(3H)-one (7aj') S54

ent-4-(2-oxopropyl)dihydrofuran-2(3H)-one (ent-8aa) S55

4-(2-oxopropyl)dihydrofuran-2(3H)-one (ent-8aa) and 2-(5-oxotetrahydrofuran-3-yl)acetaldehyde (ent-

8ac') S56

$\left[\mathrm{Ru}\left(\right.\right.$ cisoid- $\eta^{4}-2,3$-dimethylbutdiene) $\left.\left\{\eta^{4}-(1 S, 4 S)-\mathrm{Ph} 2-\mathrm{bhd} \mathbf{d}^{*}\right\}(\mathrm{NCMe})\right](\mathbf{1 0 c a}) \quad$ S56

[Ru\{ $\eta^{6}$-3-(2,3-dimethylbut-2,3-en-1-yl)-1-tosyl-2,3-dihydro-1H-pyrrole $\}\left\{\eta^{4}-(1 S, 4 S)-\mathrm{Ph}_{2}\right.$-bhd*\}] (10ca) $\mathrm{S} 57$

\section{Chromatograms}

CSP-GC Chromatograms of ent-6aa $\quad$ S58

CSP-GC Chromatogram of rac-6ab and rac-9ab $\quad$ S70

CSP-GC Chromatogram of ent-6ab and ent-9ab $\quad$ S71

CSP-GC Chromatogram of rac-6ad and rac-9ad $\quad$ S74

CSP-GC Chromatogram of ent-6ad and ent-9ad $\quad$ S76

CSP-GC Chromatogram of ent-6ae and ent-9ae S80

CSP-HPLC Chromatogram of rac-6af and rac-9af $\quad$ S81

CSP-HPLC Chromatogram of ent-6af and ent-9af $\quad$ S82

CSP-HPLC Chromatogram of rac-6ag \$83

CSP-HPLC Chromatogram of ent-6ag $\quad$ S84

CSP-GC Chromatogram of rac-7aa $\quad$ S85

CSP-GC Chromatogram of ent-7aa $\quad$ S86

CSP-GC Chromatogram of rac-7ac' $\quad$ S87

CSP-GC Chromatogram of ent-7ac' S88

CSP-GC Chromatogram of rac-7ad and rac-7ad' $\quad$ S89

CSP-GC Chromatogram of ent-7ad and ent-7ad' $\quad$ S90

CSP-HPLC Chromatogram of rac-7aj and rac-7aj' $\quad$ S93

CSP-HPLC Chromatogram of ent-7aj and ent-7aj' $\quad$ S94

S- 3 


\section{DFT Calculations}

\section{Complex 2aa}

S95

Path (S)-6aa giving (S)-6aa

S95

Path (R)-6aa giving (R)-6aa

S99

Path (R)-9af giving (R)-9af

S102

Path (S)-9af giving (S)-9af S105

Path (S)-6af giving (S)-6af S108

Path (R)-6af giving (R)-6af

S111

S- 4 


\section{Spectroscopic Charts}

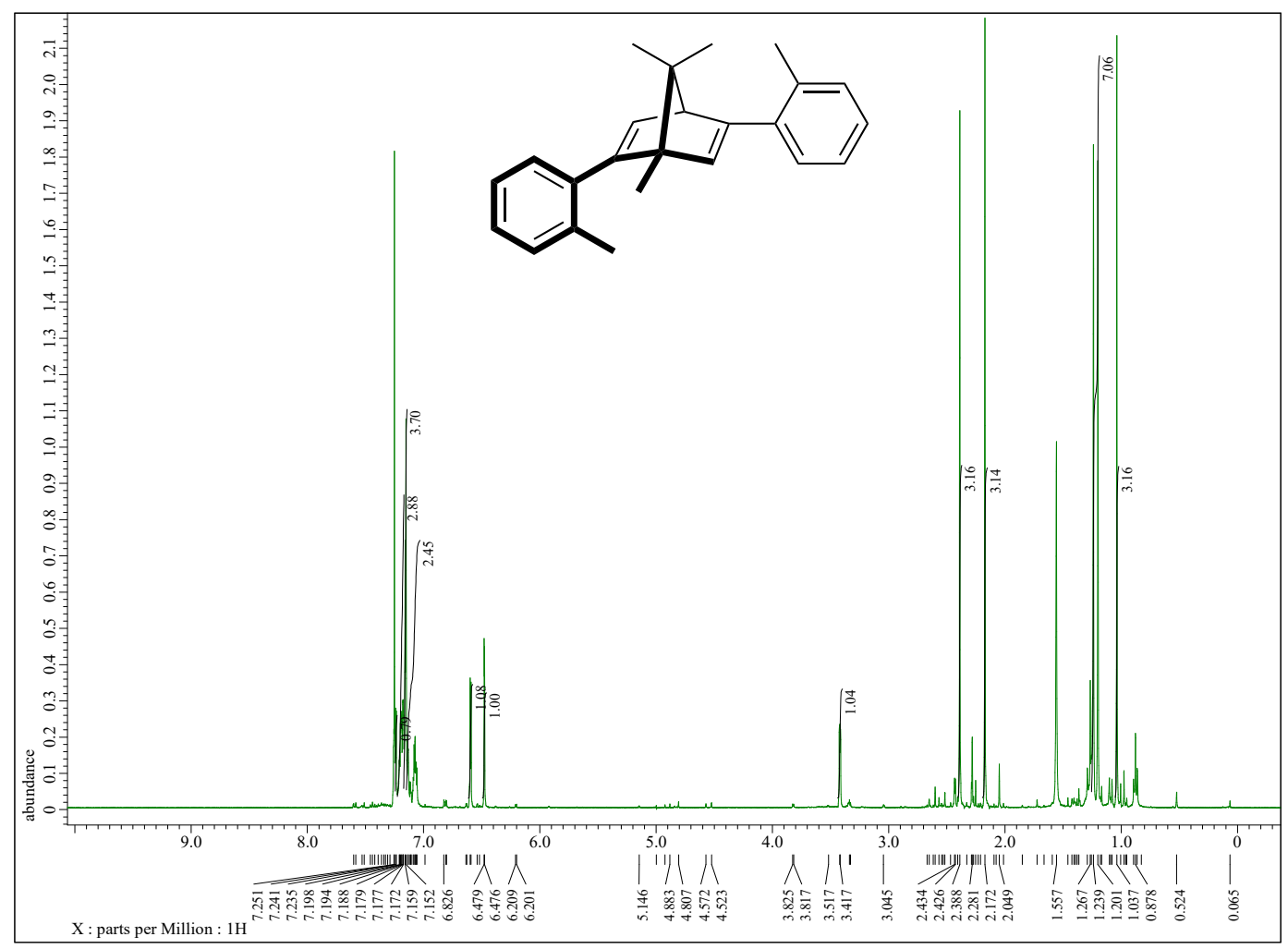

Figure S1-1. ' $\mathrm{H}$ NMR Spectrum of (1S,4S)-(2-Tol) ${ }_{2}$-bhd* $\left(400 \mathrm{MHz}, \mathrm{CDCl}_{3}\right.$, r.t.).

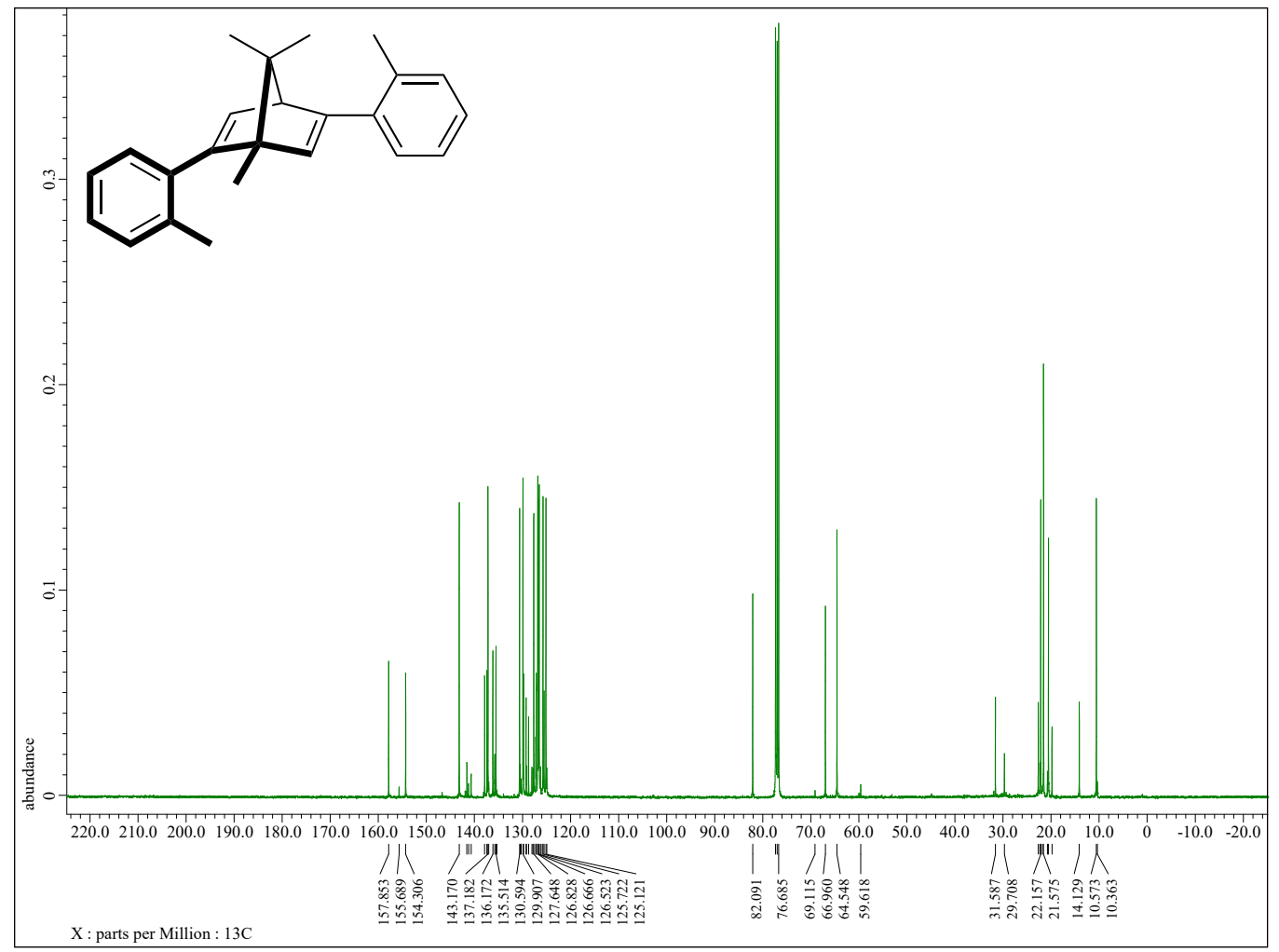

Figure S1-2. ${ }^{13} \mathrm{C}\left\{{ }^{1} \mathrm{H}\right\}$ NMR Spectrum of $(1 S, 4 S)-\left(2-\mathrm{Tol}_{2}\right)_{2}$-bhd ${ }^{*}\left(100 \mathrm{MHz}, \mathrm{CDCl}{ }_{3}\right.$, r.t.). 


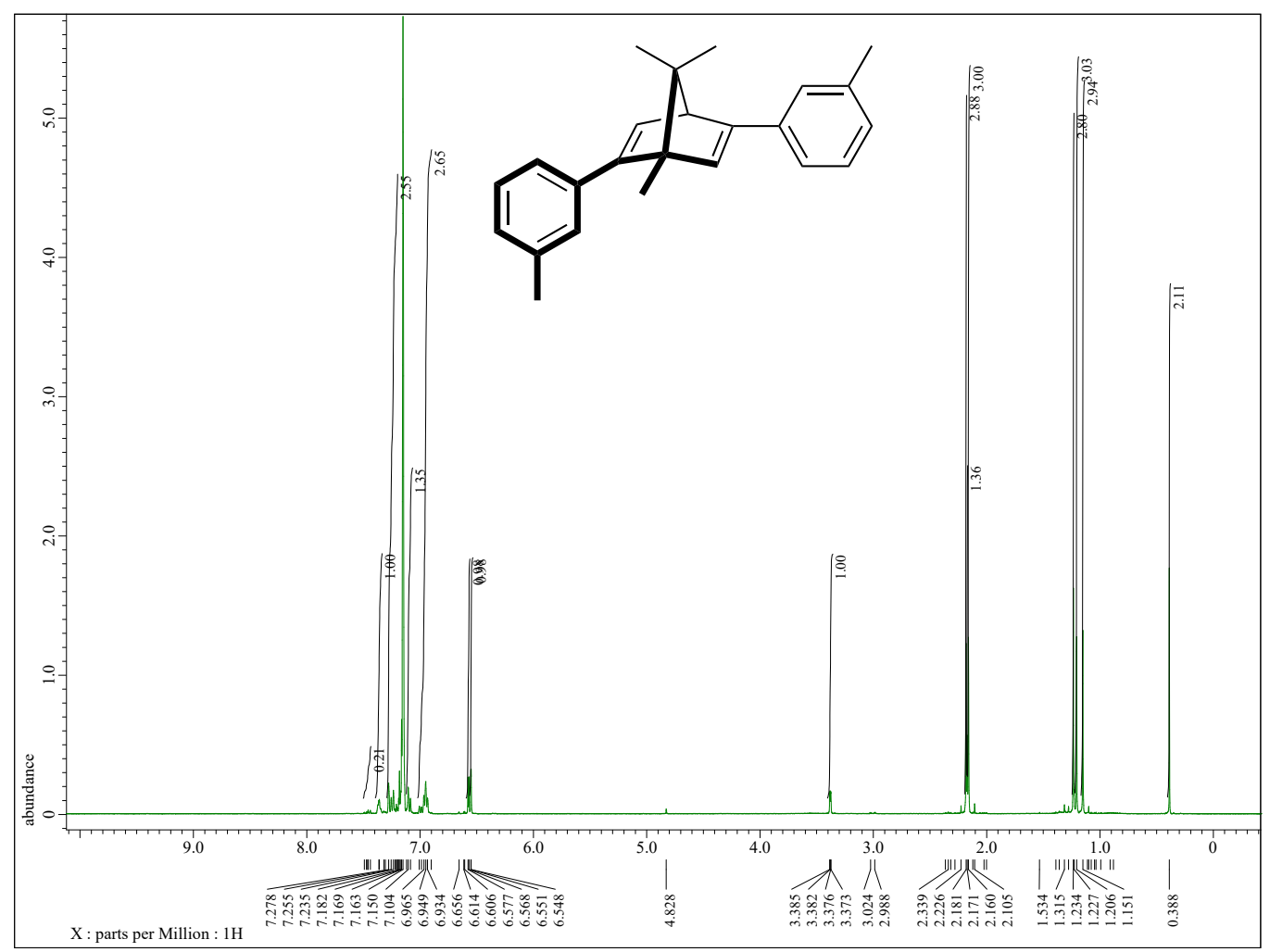

Figure S2-1. ${ }^{1} \mathrm{H}$ NMR of Spectrum $(1 \mathrm{~S}, 4 \mathrm{~S})-(3-\mathrm{Tol})_{2}$-bhd* $\left(400 \mathrm{MHz}, \mathrm{CDCl}_{3}\right.$, r.t.).

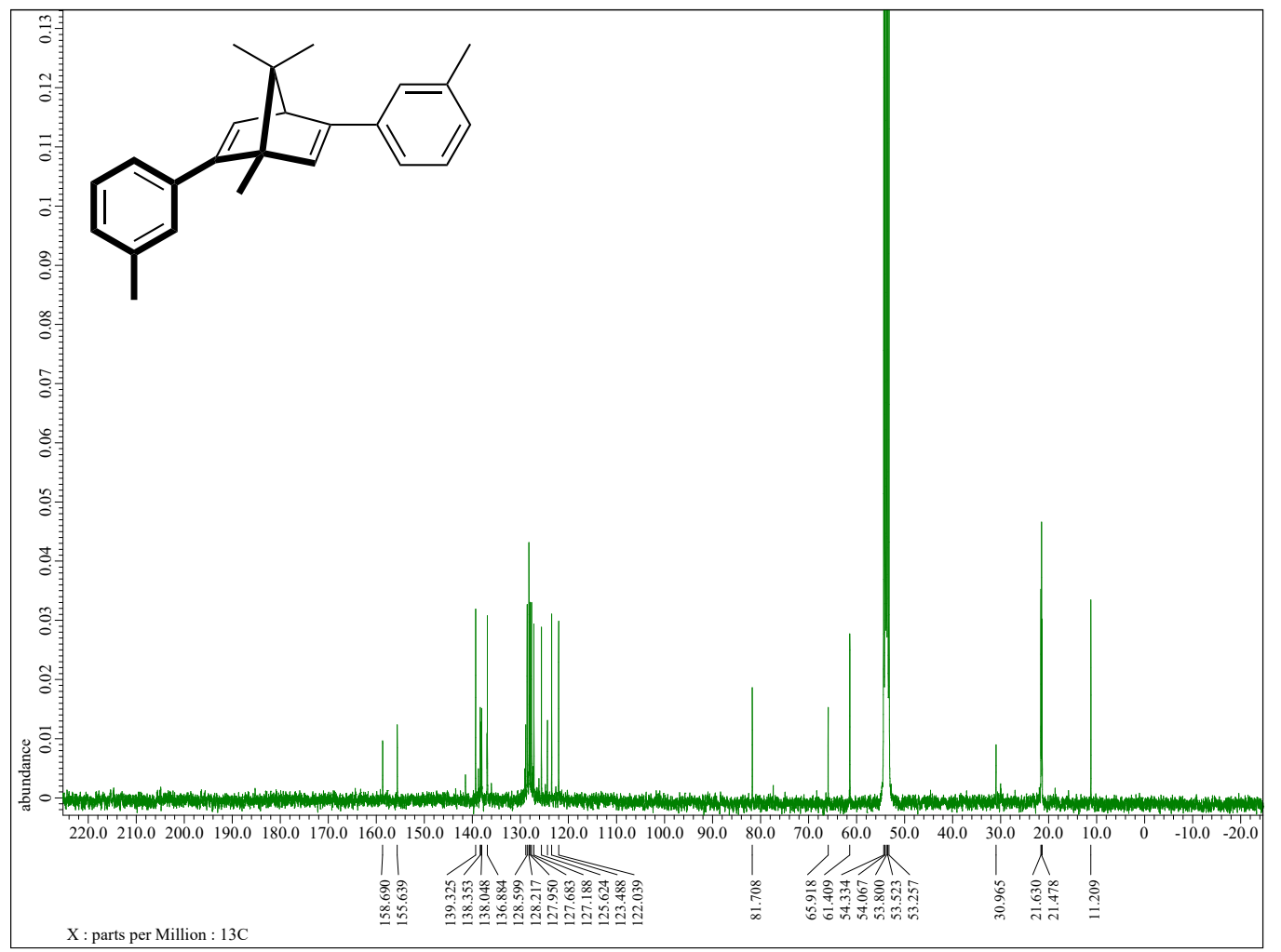

Figure S2-2. ${ }^{13} \mathrm{C}\left\{{ }^{1} \mathrm{H}\right\}$ NMR Spectrum of $(1 \mathrm{~S}, 4 \mathrm{SS})-(3-\mathrm{Tol})_{2}$-bhd ${ }^{*}\left(100 \mathrm{MHz}, \mathrm{CD}_{2} \mathrm{Cl}_{2}\right.$, r.t. $)$ 


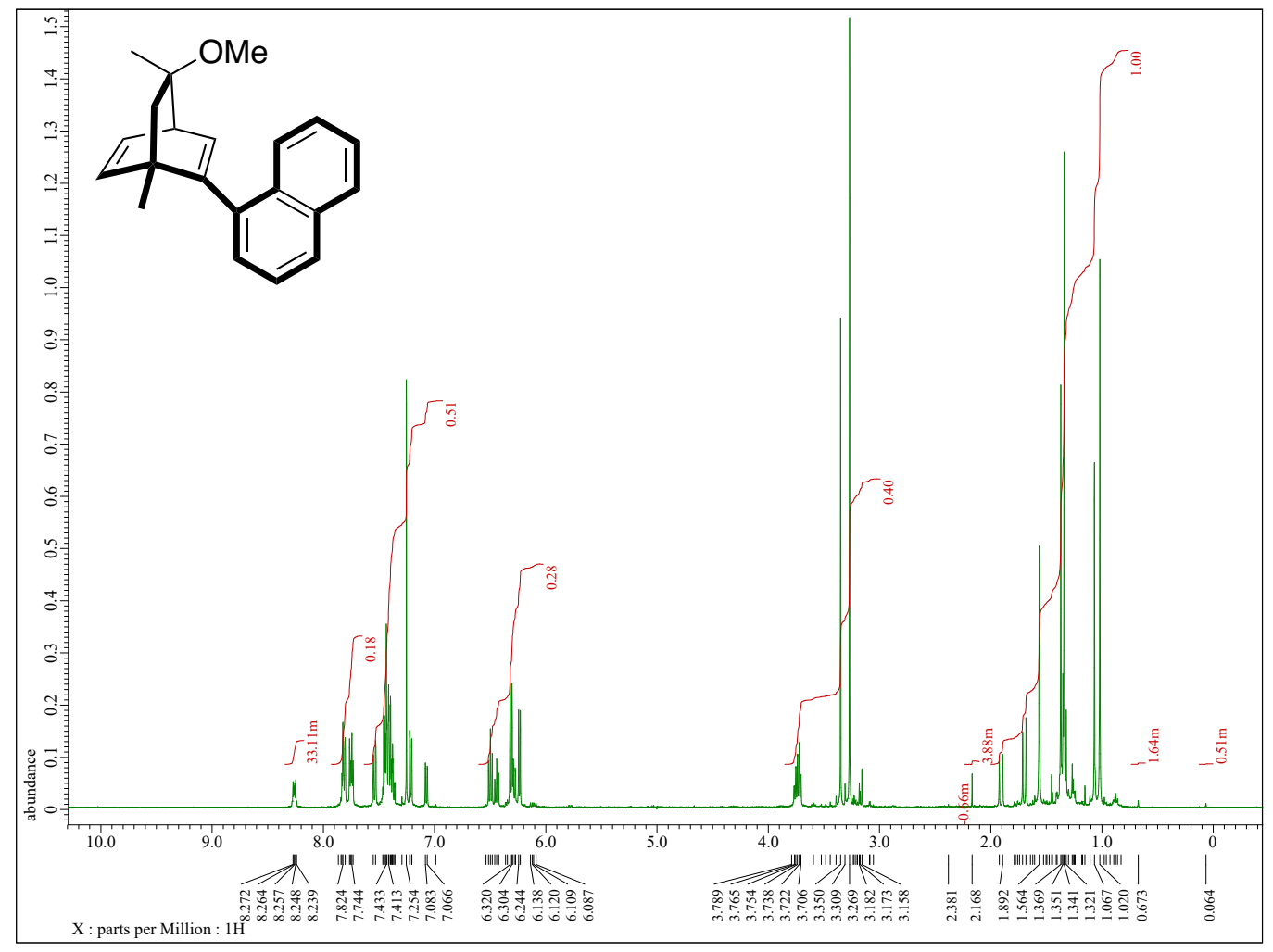

Figure S3-1. ' $\mathrm{H}$ NMR Spectrum of (1S,4R,8R)-(1-Nap)-bod* (400 MHz, $\mathrm{CDCl}_{3}$, r.t.).

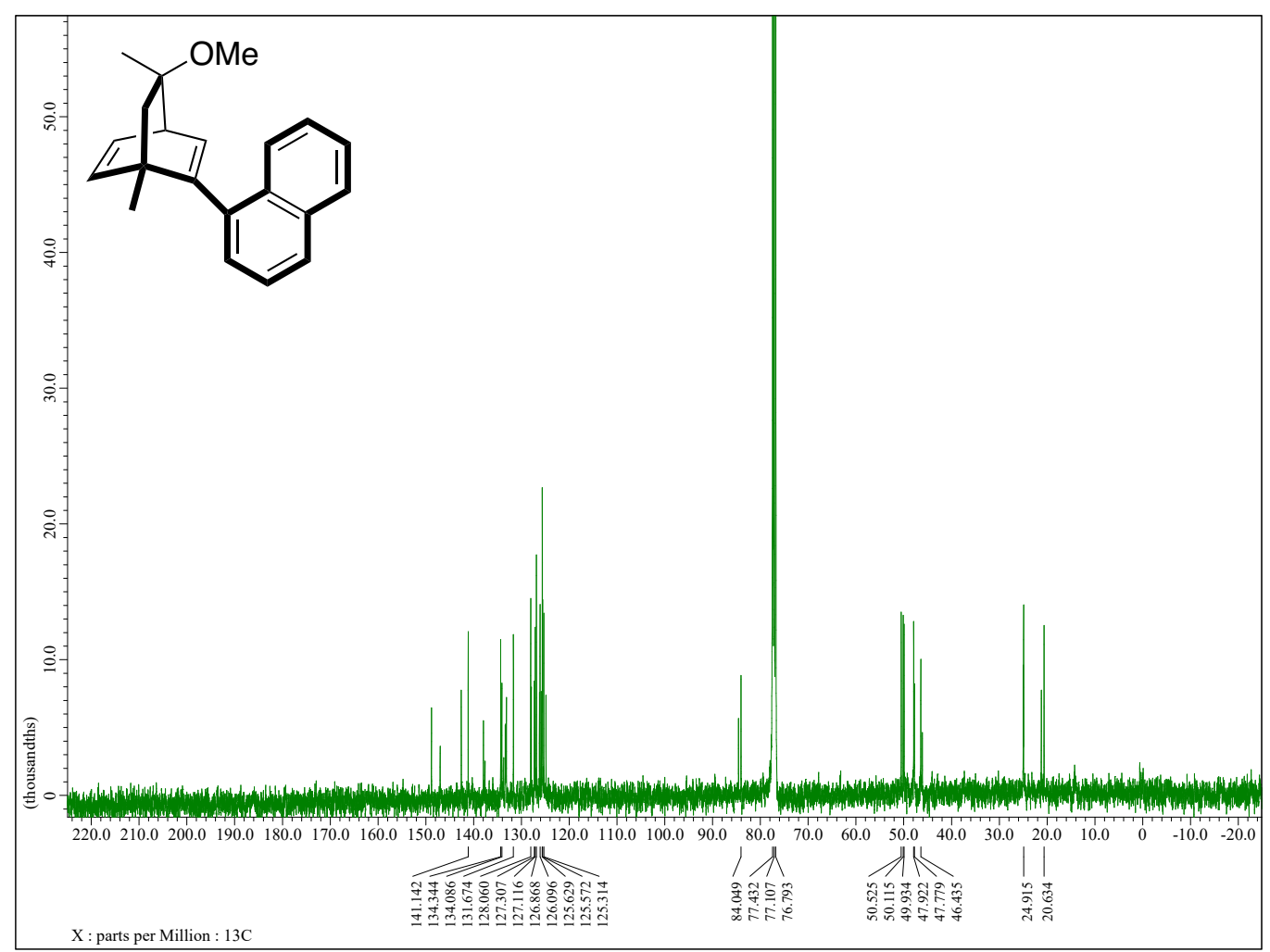

Figure S3-2. ${ }^{13} \mathrm{C}\{1 \mathrm{H}\}$ NMR Spectrum of $(1 \mathrm{~S}, 4 R, 8 R)$-(1-Nap)-bod* $\left(100 \mathrm{MHz}, \mathrm{CDCl}_{3}\right.$, r.t.). 


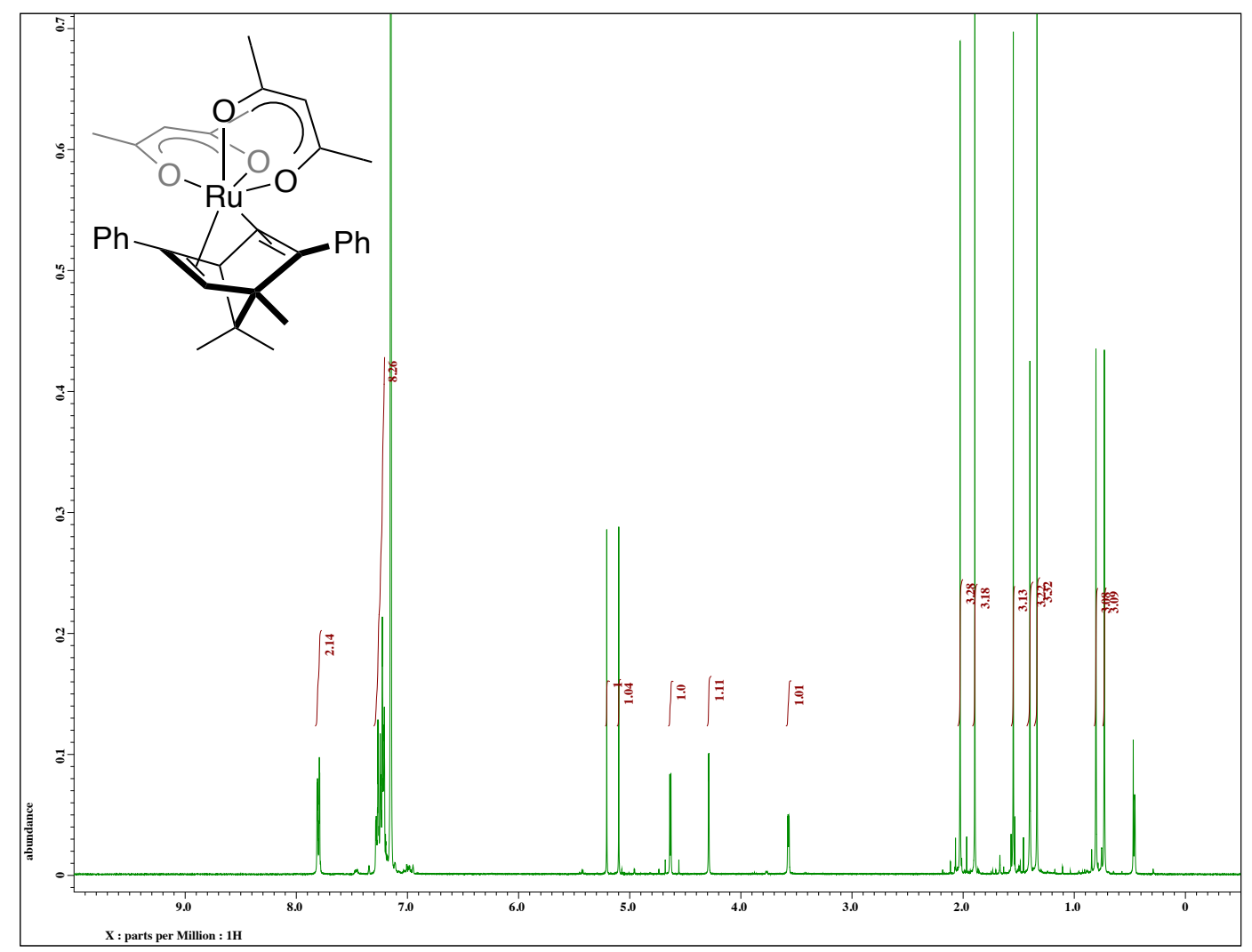

Figure S4-1. ${ }^{1} \mathrm{H}$ NMR Spectrum of $\Delta-\left[\mathrm{Ru}(\mathrm{acac})_{2}\left\{\eta^{4}-(1 S, 4 S)-\mathrm{Ph}_{2}-\mathrm{bhd}^{*}\right\}\right](\Delta-1 \mathbf{a})\left(400 \mathrm{MHz}, \mathrm{CDCl}_{3}\right.$, r.t.).

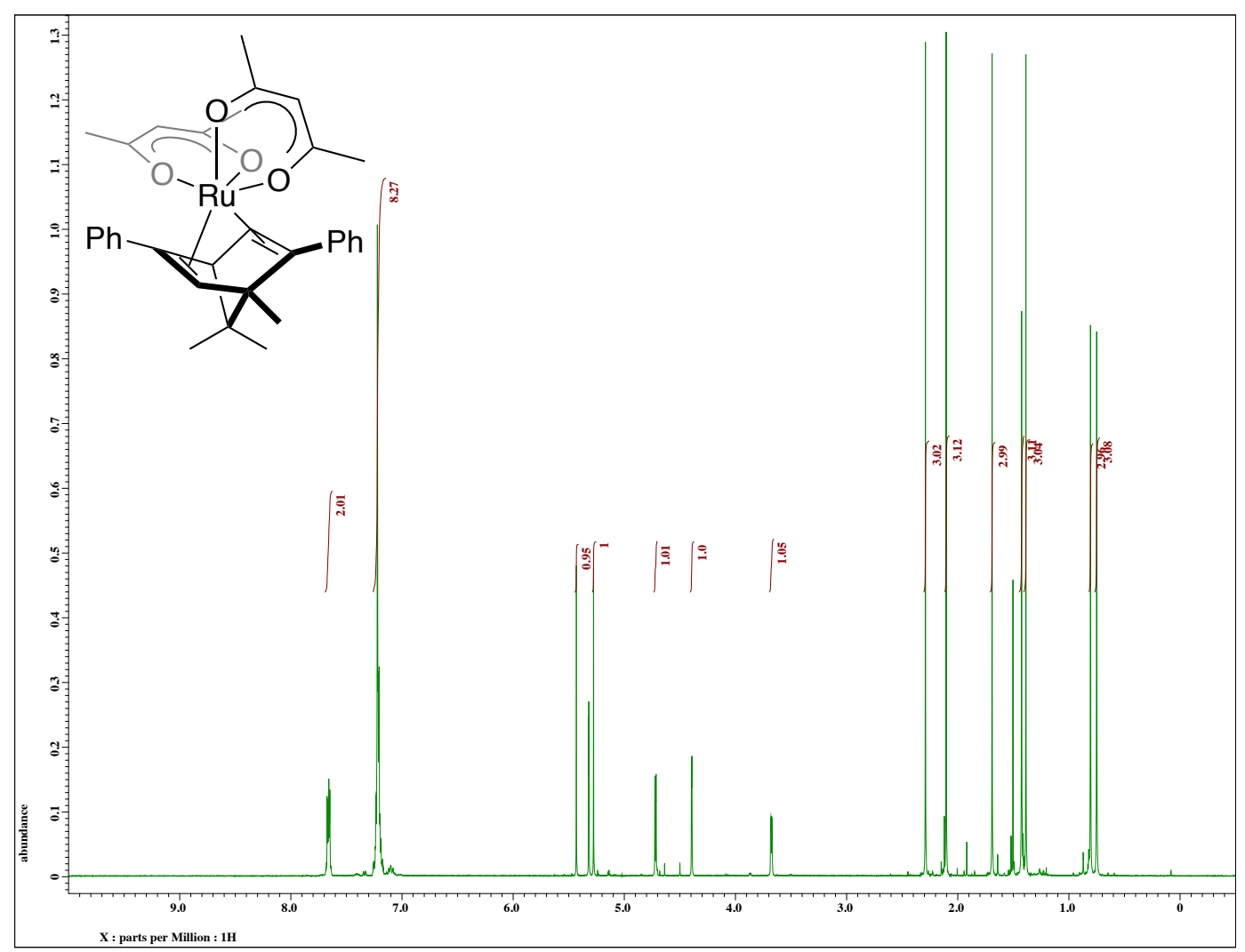

Figure S4-2. ${ }^{1} \mathrm{H}$ NMR Spectrum of $\Delta-\left[\mathrm{Ru}(\mathrm{acac})_{2}\left\{\eta^{4}-(1 S, 4 S)-\mathrm{Ph}_{2}\right.\right.$-bhd* $\left.\}\right]\left(\Delta-\mathbf{1}\right.$ a) $\left(400 \mathrm{MHz}, \mathrm{CD}_{2} \mathrm{Cl}_{2}\right.$, r.t.). 


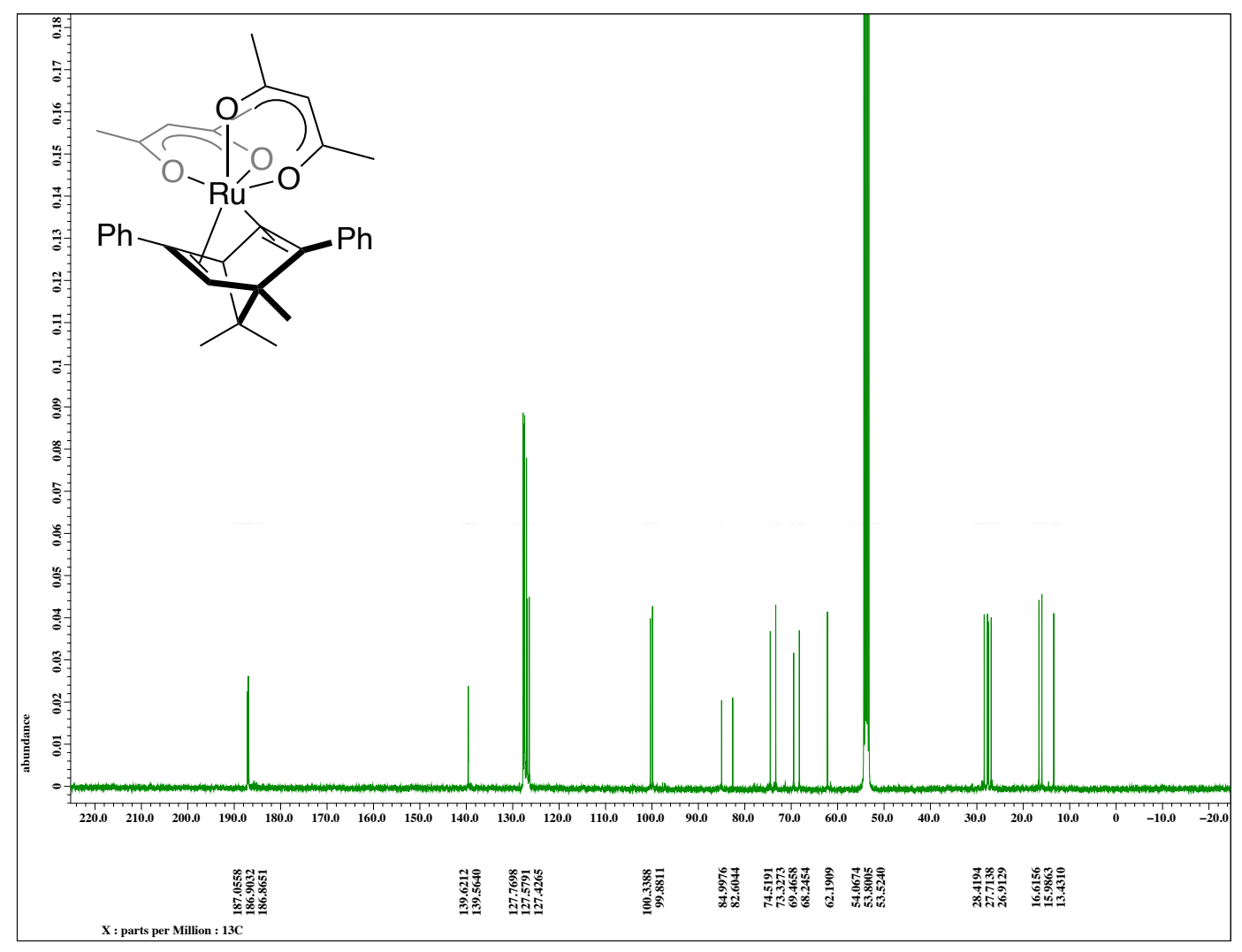

Figure S4-3. ${ }^{13} \mathrm{C}\left\{{ }^{1} \mathrm{H}\right\}$ NMR Spectrum of $\Delta$-[Ru(acac $)_{2}\left\{\eta^{4}-(1 \mathrm{~S}, 4 S)-\mathrm{Ph}_{2}\right.$-bhd $\left.\}\right]\left(\Delta-1\right.$ a) $\left(100 \mathrm{MHz}, \mathrm{CD}_{2} \mathrm{Cl}_{2}\right.$, r.t.).

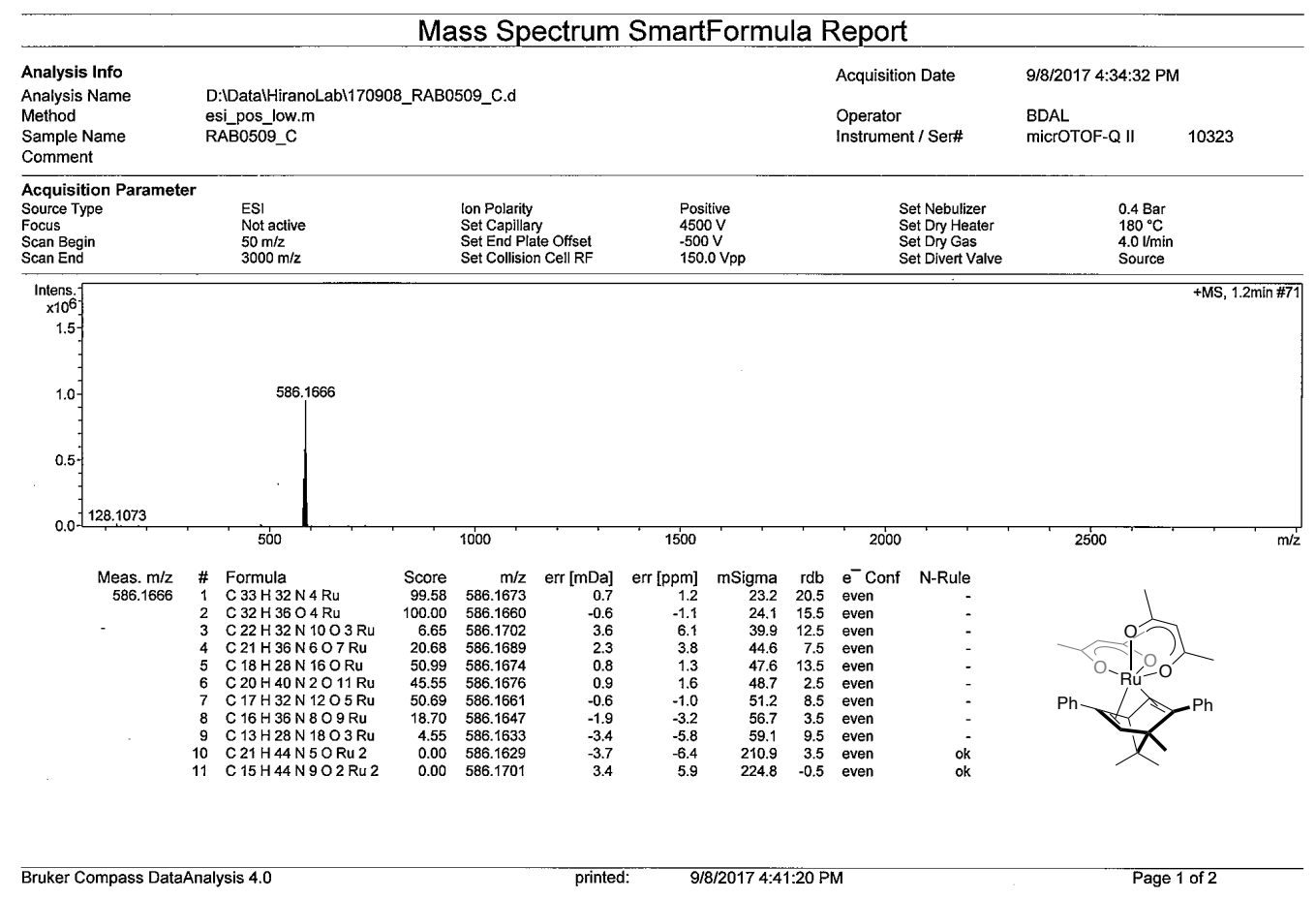

Figure S4-4. HRMS (ESI) Spectrum of $\Delta-\left[R u(\operatorname{acac})_{2}\left\{\eta^{4}-(1 S, 4 S)-\mathrm{Ph}_{2}-\mathrm{bhd}^{*}\right\}\right](\Delta-1 \mathbf{a})$. 


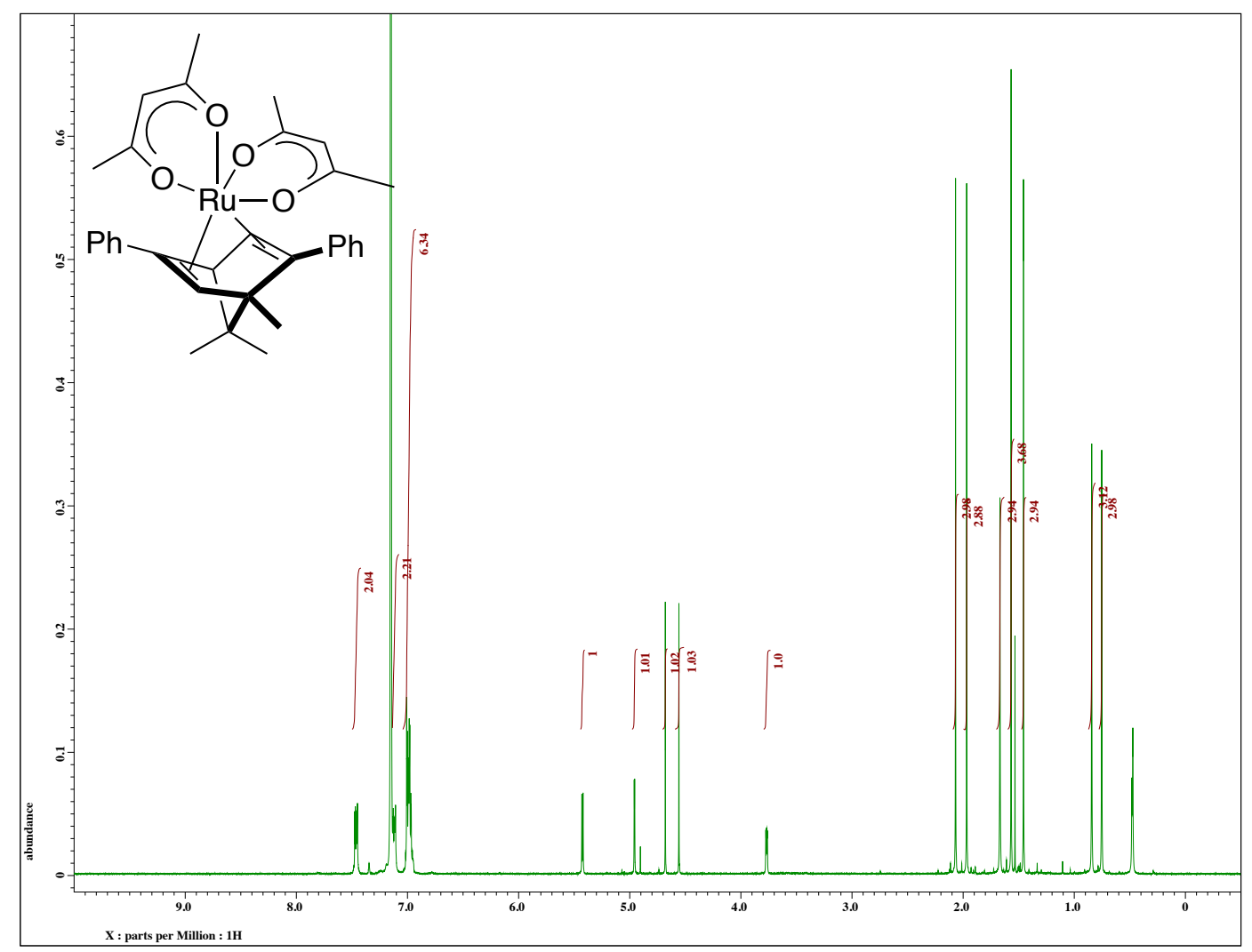

Figure S4-5. ${ }^{1} \mathrm{H}$ NMR Spectrum of $\Lambda$-[Ru(acac) ${ }_{2}\left\{\eta^{4}-(1 S, 4 S)-\mathrm{Ph}_{2}\right.$-bhd $\left.\left.{ }^{*}\right\}\right](\Lambda-1 \mathbf{a})\left(400 \mathrm{MHz}, \mathrm{CDCl}_{3}\right.$, r.t.).

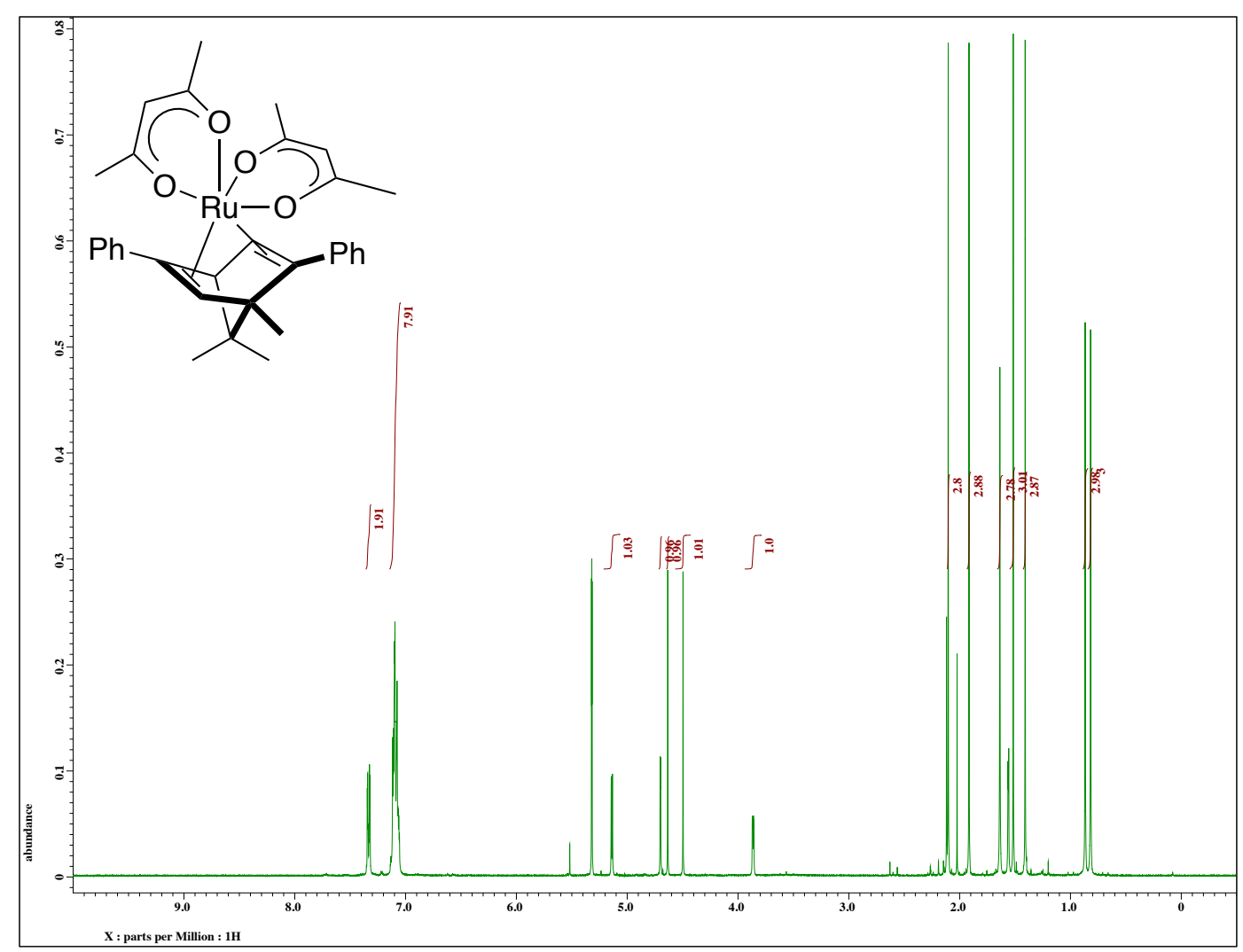

Figure S4-6. ${ }^{1} \mathrm{H}$ NMR Spectrum of $\Lambda$-[Ru(acac) $\left.{ }_{2}\left\{\eta^{4}-(1 S, 4 S)-\mathrm{Ph}_{2}-\mathrm{bhd}^{*}\right\}\right](\Lambda-1 \mathrm{a})\left(400 \mathrm{MHz}, \mathrm{CD}_{2} \mathrm{Cl}_{2}\right.$, r.t. $)$ 


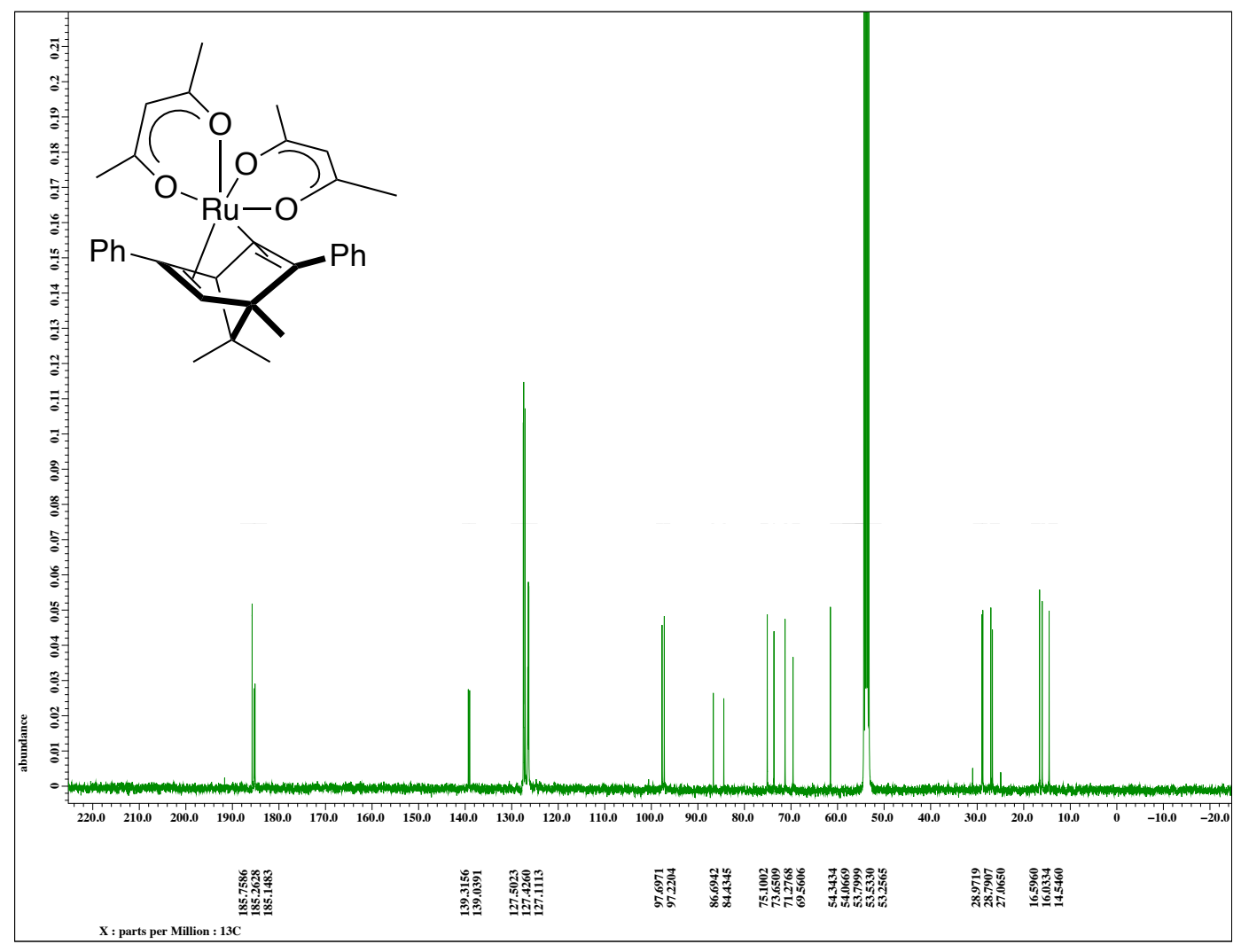

Figure S4-7. ${ }^{13} \mathrm{C}\left\{{ }^{1} \mathrm{H}\right\}$ NMR Spectrum of $\Lambda$ - $\left[\mathrm{Ru}(\text { acac })_{2}\left\{\eta^{4}-(1 S, 4 S)-\mathrm{Ph}_{2}-\right.\right.$ bhd $\left.\left.d^{*}\right\}\right](\Lambda-1 \mathbf{a})\left(100 \mathrm{MHz}, \mathrm{CD}_{2} \mathrm{Cl}_{2}\right.$, r.t.).

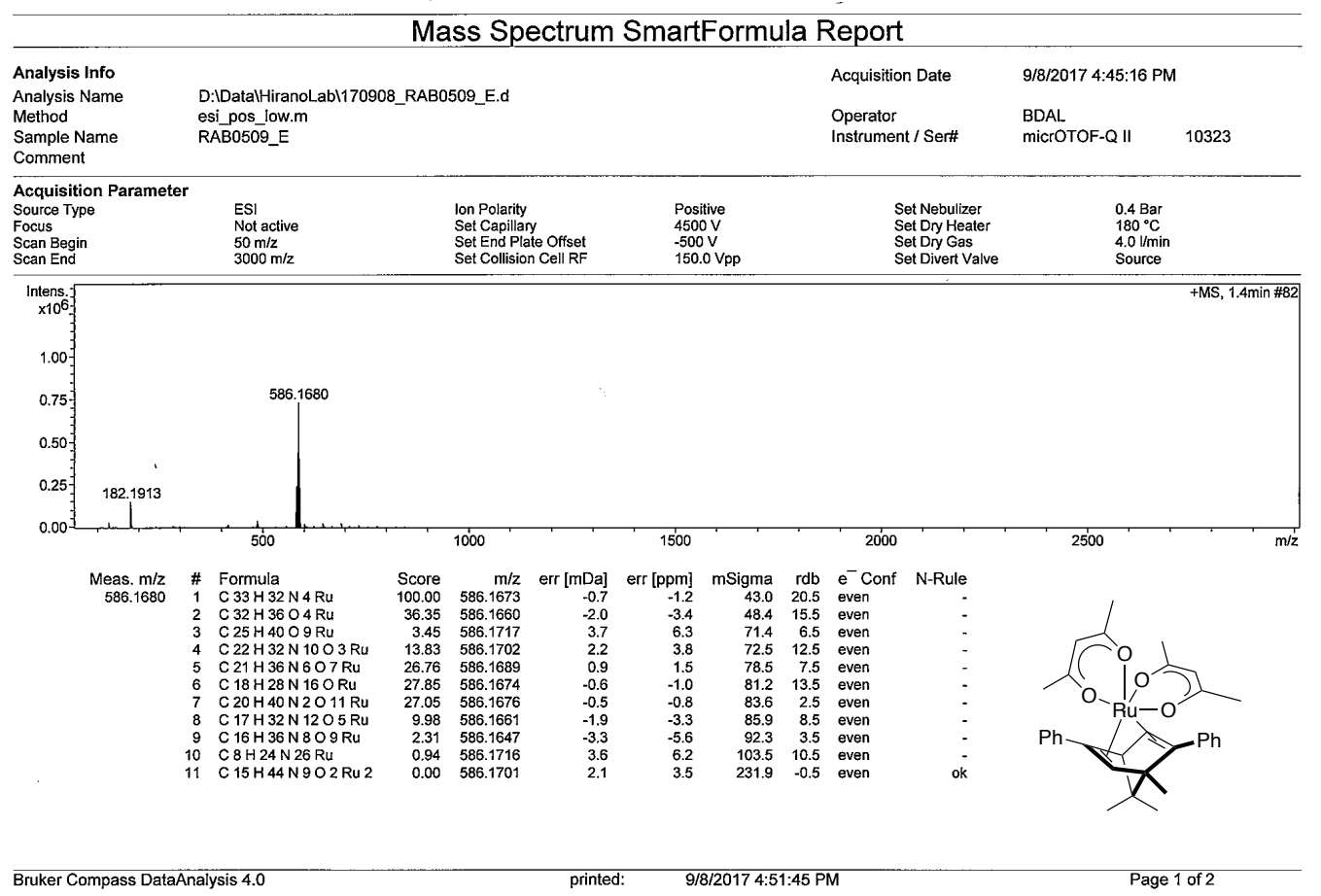

Figure S4-8. HRMS (ESI) Spectrum of $\Lambda$-[Ru(acac $\left.)_{2}\left\{\eta^{4}-(1 S, 4 S)-\mathrm{Ph}_{2}-\mathrm{bhd}^{*}\right\}\right](\Lambda-1 \mathbf{a})$. 


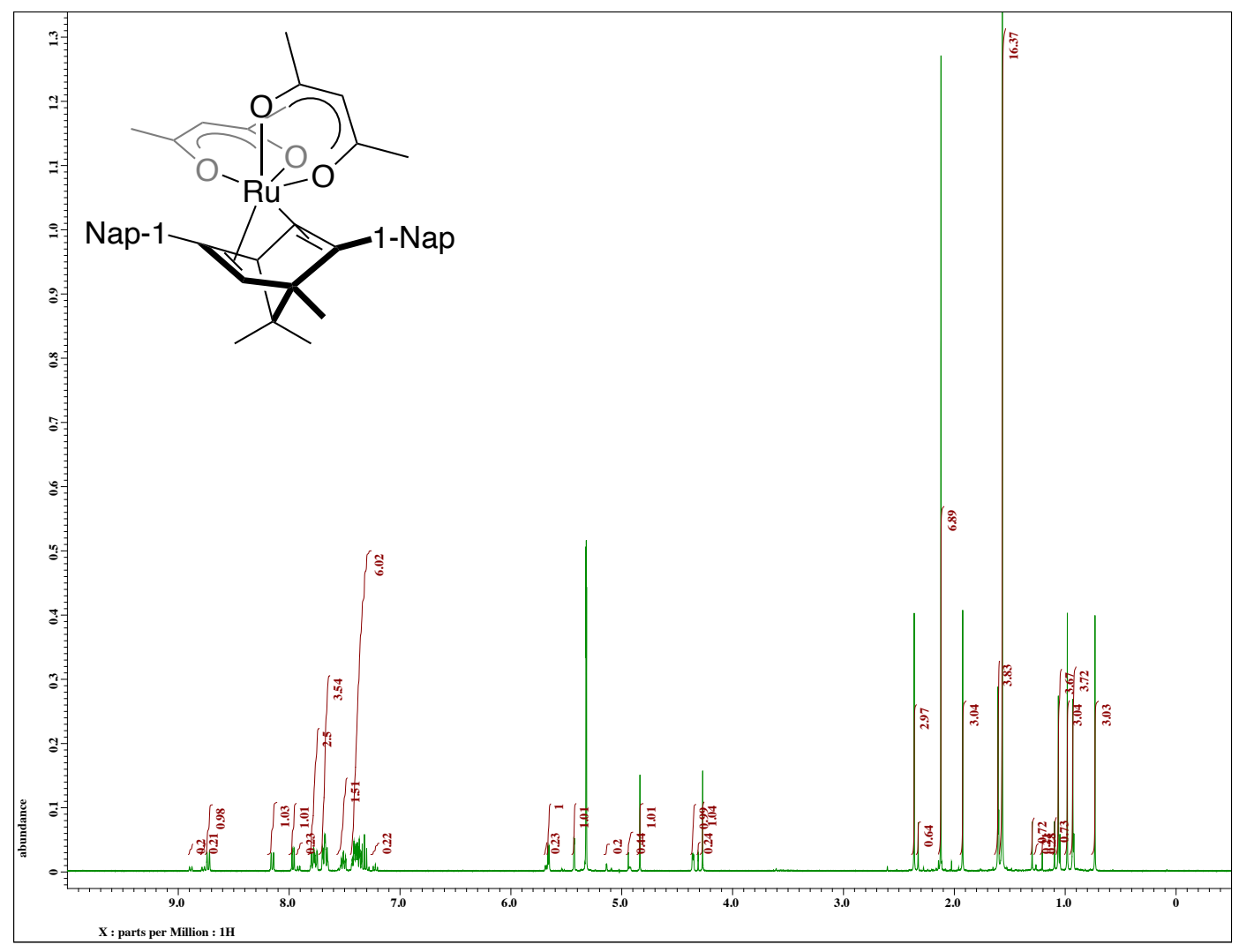

Figure S5-1. ${ }^{1} \mathrm{H}$ NMR Spectrum of $\left[\mathrm{Ru}(\mathrm{acac})_{2}\left\{\eta^{4}-(1 \mathrm{~S}, 4 \mathrm{~S})-(1-\mathrm{Nap})_{2}-\right.\right.$ bhd $\left.\left.\mathrm{d}^{*}\right\}\right](\mathbf{1} \mathbf{b})\left(400 \mathrm{MHz}, \mathrm{CD}_{2} \mathrm{Cl}_{2}\right.$, r.t.).

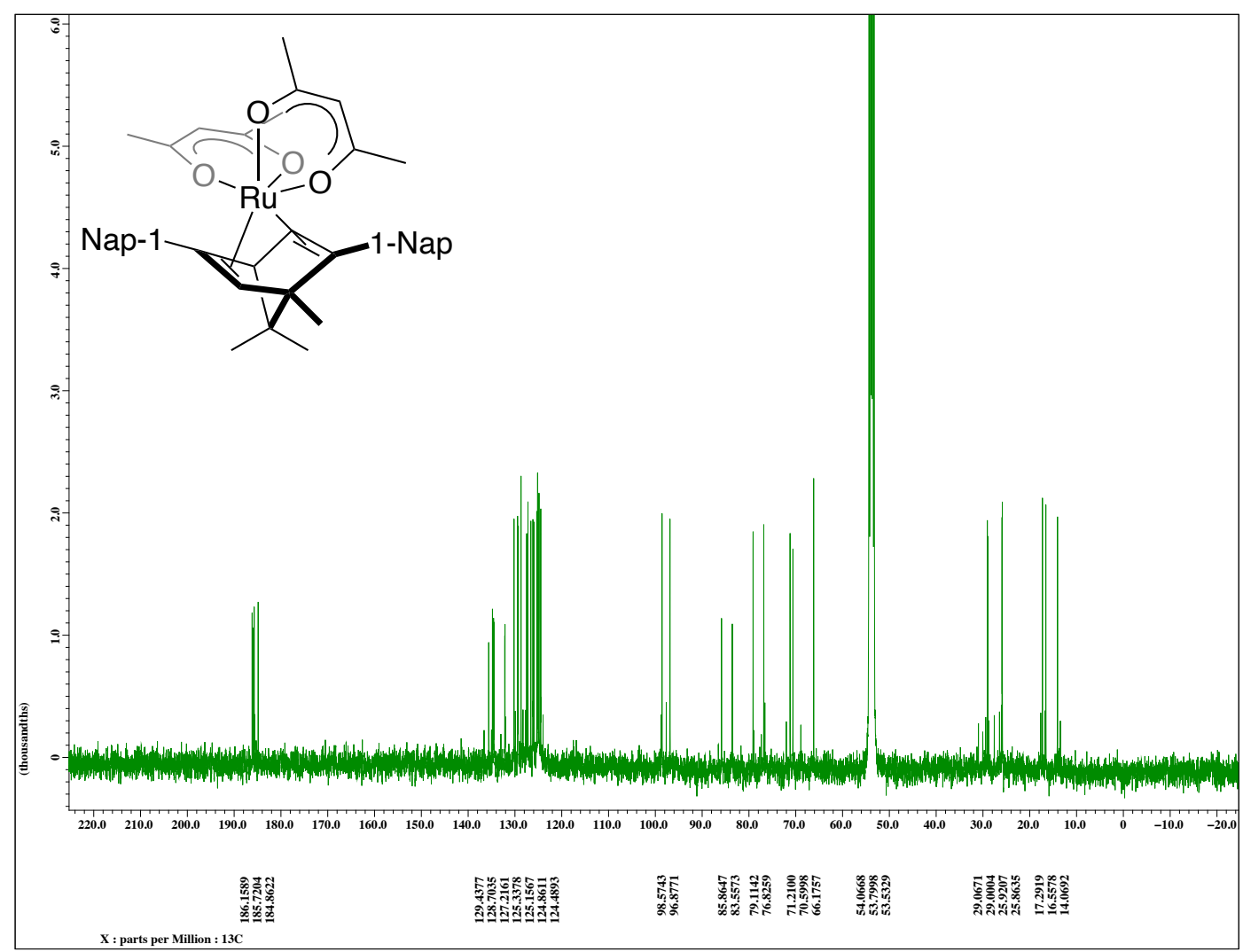

Figure S5-2. ${ }^{13} \mathrm{C}\left\{{ }^{1} \mathrm{H}\right\}$ NMR Spectrum of $\left[\mathrm{Ru}(\mathrm{acac})_{2}\left\{\eta^{4}-(1 \mathrm{~S}, 4 \mathrm{~S})-(1-\mathrm{Nap})_{2}-\mathrm{bhd}^{*}\right\}\right](\mathbf{1 b})\left(100 \mathrm{MHz}, \mathrm{CD}_{2} \mathrm{Cl}_{2}\right.$, r.t.). 
Mass Spectrum SmartFormula Report

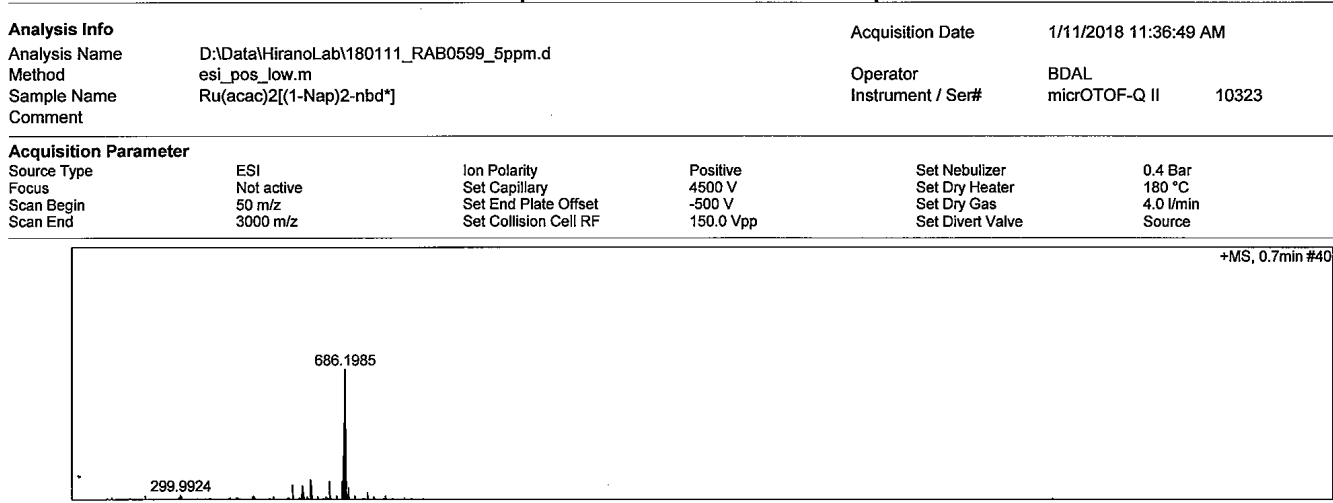

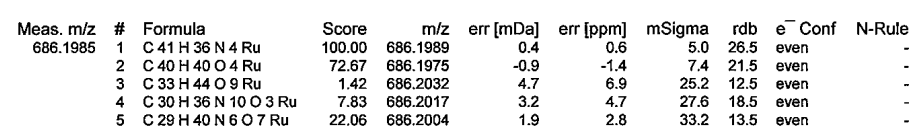

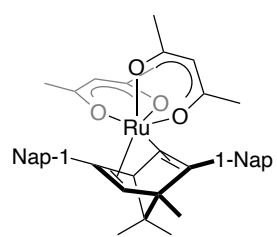

Bruker Compass DataAnalysis 4.0

printed: $\quad$ 1/11/2018 11:41:59 AM

Page 1 of 2

Figure S5-3. HRMS (ESI) Spectrum of $\left[\mathrm{Ru}(\mathrm{acac})_{2}\left\{\eta^{4}-(1 S, 4 S)-(1-\mathrm{Nap})_{2}-\mathrm{bhd}^{*}\right\}\right](\mathbf{1 b})$. 


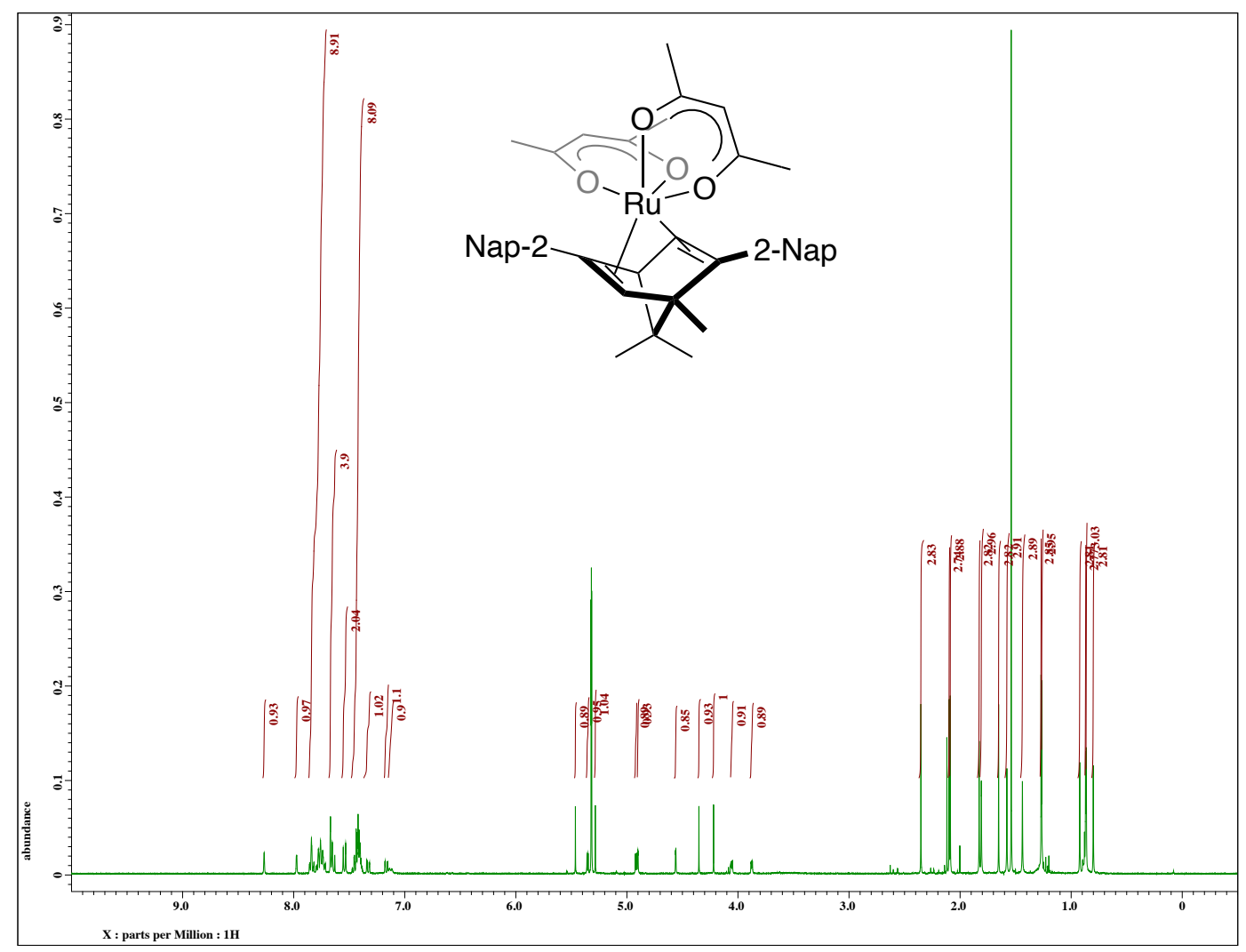

Figure S6-1. ${ }^{1} \mathrm{H}$ NMR Spectrum of $\left[\mathrm{Ru}(\mathrm{acac})_{2}\left\{\eta^{4}-(1 \mathrm{~S}, 4 S)-(2-\mathrm{Nap})_{2}-\mathrm{bhd}^{*}\right\}\right](\mathbf{1 c})\left(400 \mathrm{MHz}, \mathrm{CD}_{2} \mathrm{Cl}_{2}\right.$, r.t.).

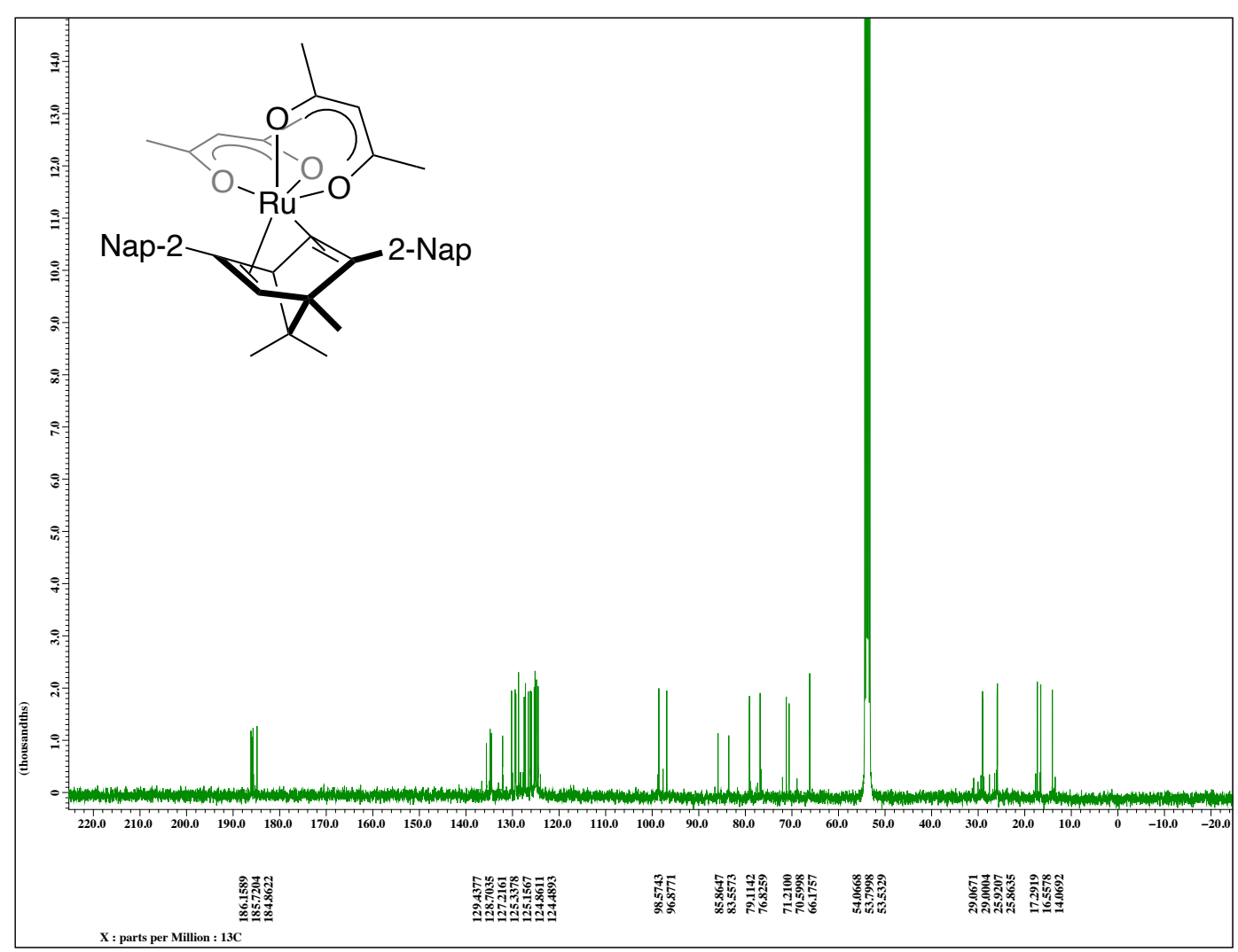

Figure S6-2. ${ }^{13} \mathrm{C}\left\{{ }^{1} \mathrm{H}\right\}$ NMR Spectrum of $\left[\mathrm{Ru}(\mathrm{acac})_{2}\left\{\eta^{4}-(1 \mathrm{~S}, 4 S)-(2-\mathrm{Nap})_{2}-\mathrm{bhd} \mathbf{B}^{*}\right](\mathbf{1} \mathbf{c})\left(100 \mathrm{MHz}, \mathrm{CD}_{2} \mathrm{Cl}_{2}\right.\right.$, r.t.). 
Mass Spectrum SmartFormula Report

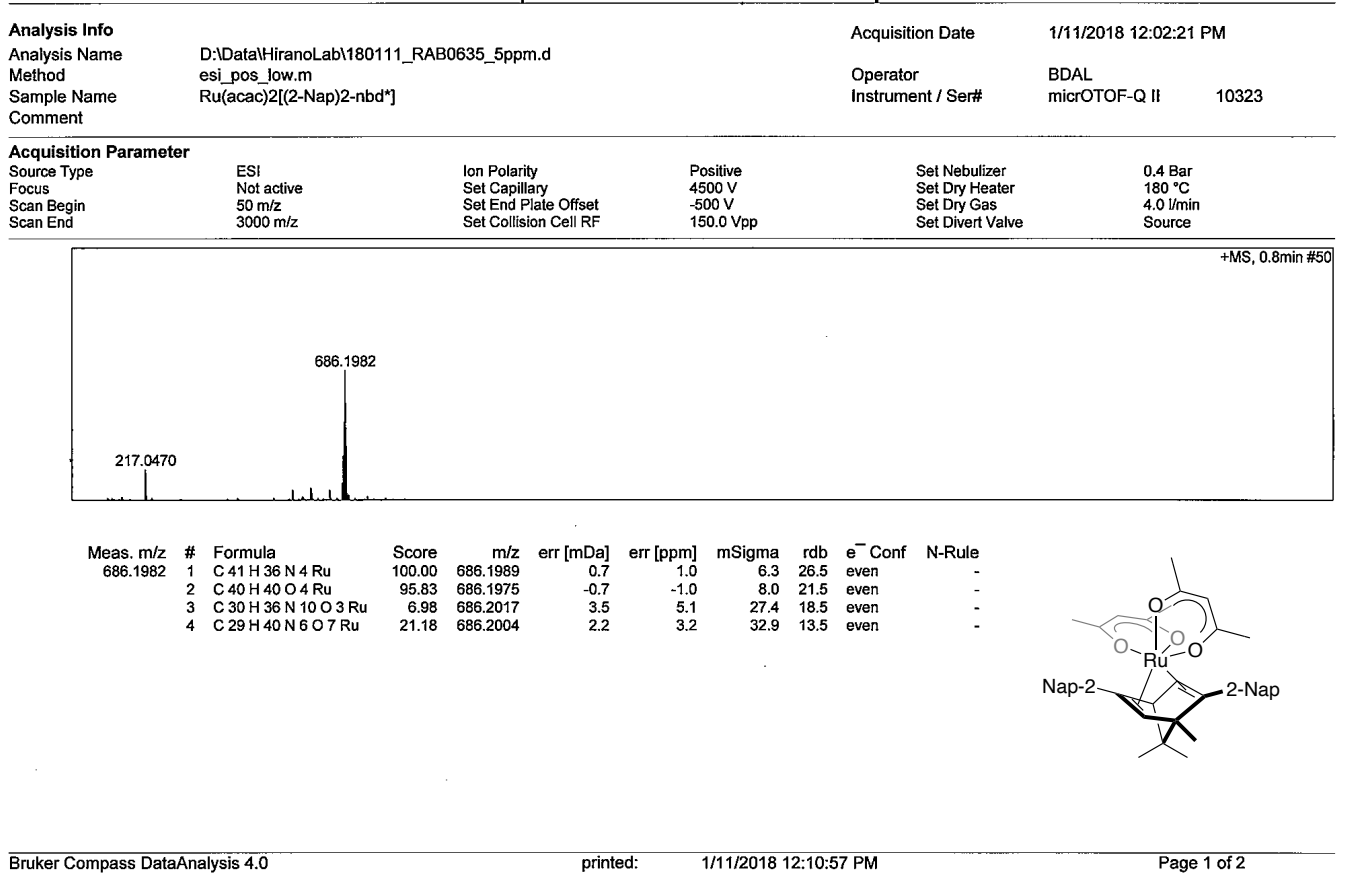

Figure S6-3. HRMS (ESI) Spectrum of $\left[\mathrm{Ru}(\mathrm{acac})_{2}\left\{\eta^{4}-(1 S, 4 S)-(2-\mathrm{Nap})_{2}-\right.\right.$ bhd $\left.\left.d^{*}\right\}\right]$ (1c). 


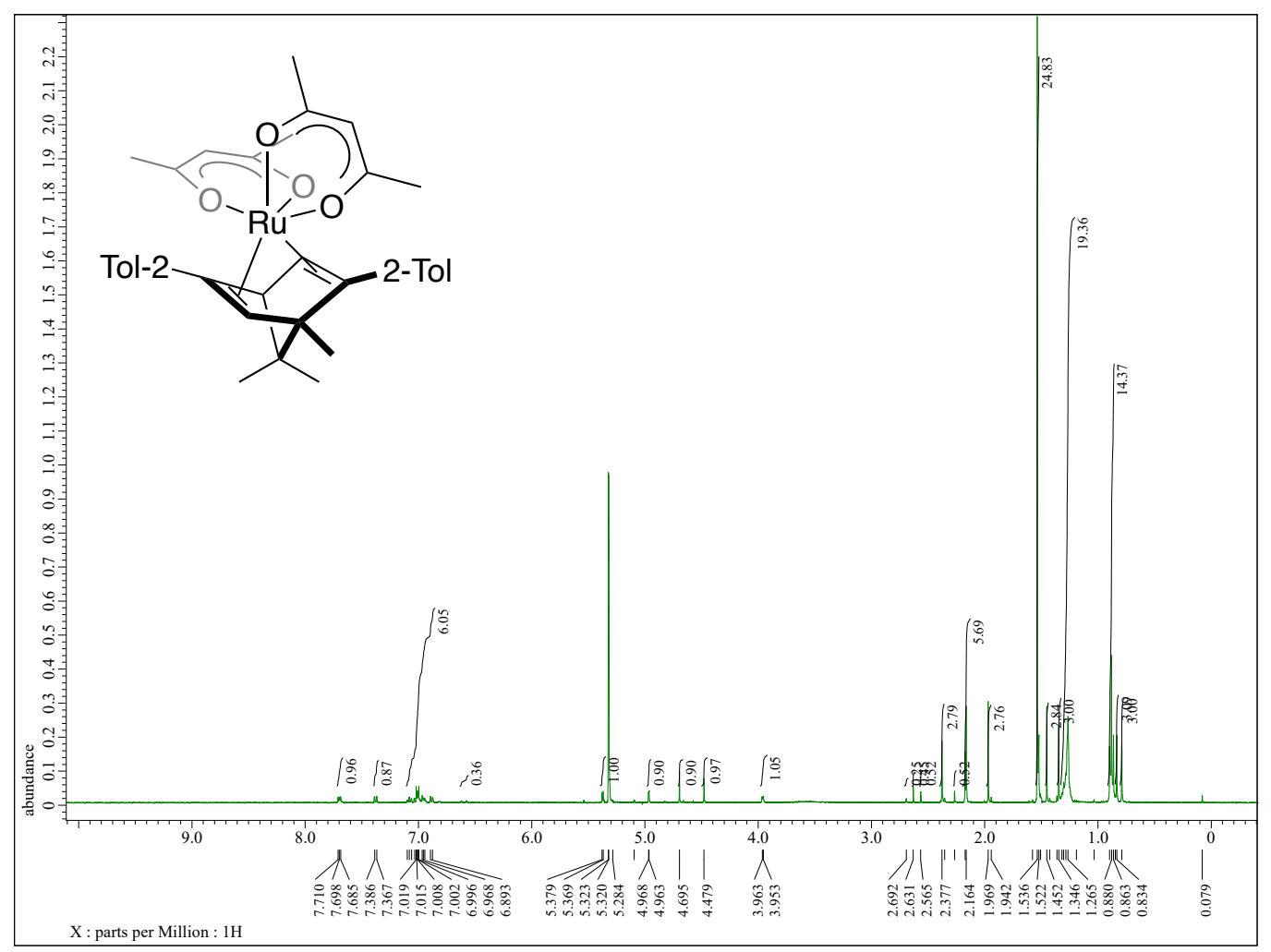

Figure S7-1. ${ }^{1} \mathrm{H}$ NMR of Spectrum of $\left[\mathrm{Ru}(\mathrm{acac})_{2}\left\{\eta^{4}-(1 \mathrm{~S}, 4 S)-\left(2-\mathrm{Tol}_{2}\right)_{2}\right.\right.$-bhd $\left.\left.{ }^{*}\right\}\right]$ (1d). $\left(400 \mathrm{MHz}, \mathrm{CD}_{2} \mathrm{Cl}_{2}\right.$, r.t.).

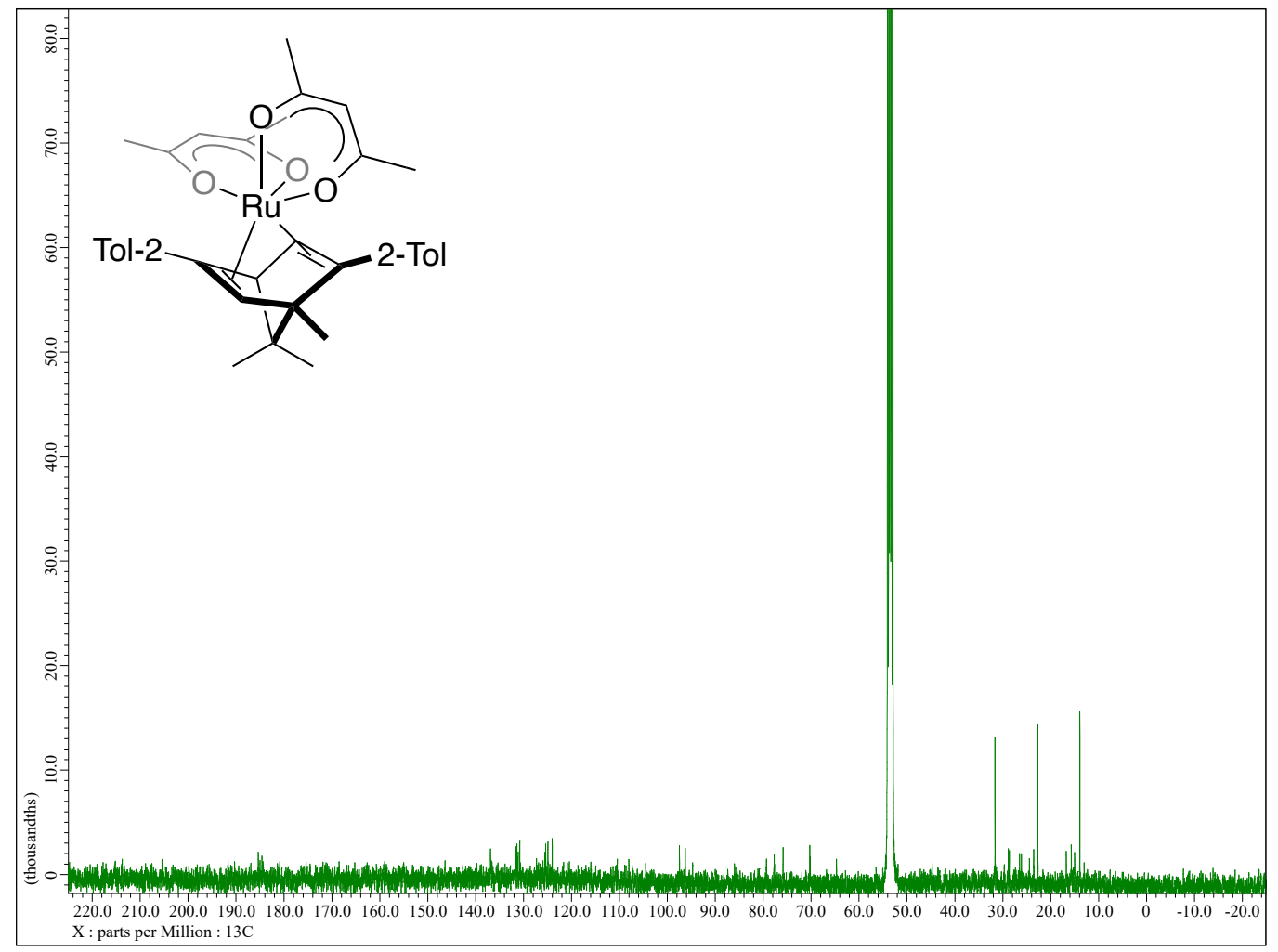

Figure S7-2. ${ }^{13} \mathrm{C}\left\{{ }^{1} \mathrm{H}\right\} \mathrm{NMR}$ Spectrum of $\left[\mathrm{Ru}(\mathrm{acac})_{2}\left\{\eta^{4}-(1 \mathrm{~S}, 4 \mathrm{~S})-(2-\mathrm{Tol})_{2}-\mathrm{bhd}{ }^{*}\right\}\right]$ (1d) $\left(100 \mathrm{MHz}, \mathrm{CD}_{2} \mathrm{Cl}_{2}\right.$, r.t.). 
Mass Spectrum SmartFormula Report

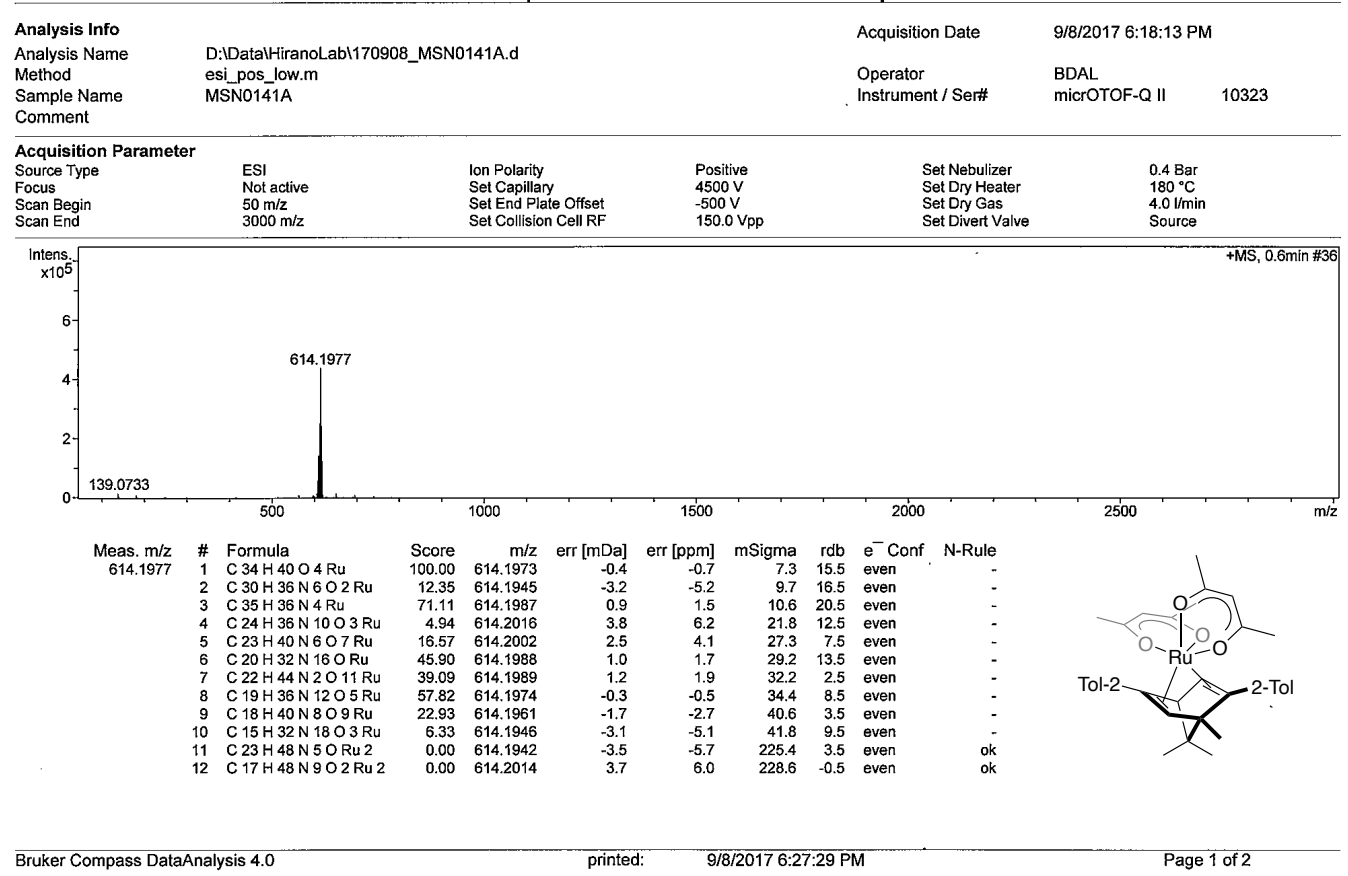

Figure S7-3. HRMS (ESI) Spectrum of $\left[\mathrm{Ru}(\mathrm{acac})_{2}\left\{\eta^{4}-(1 S, 4 S)-(2-\mathrm{Tol})_{2}\right.\right.$-bhd* $\left.\}\right]$ (1d). 


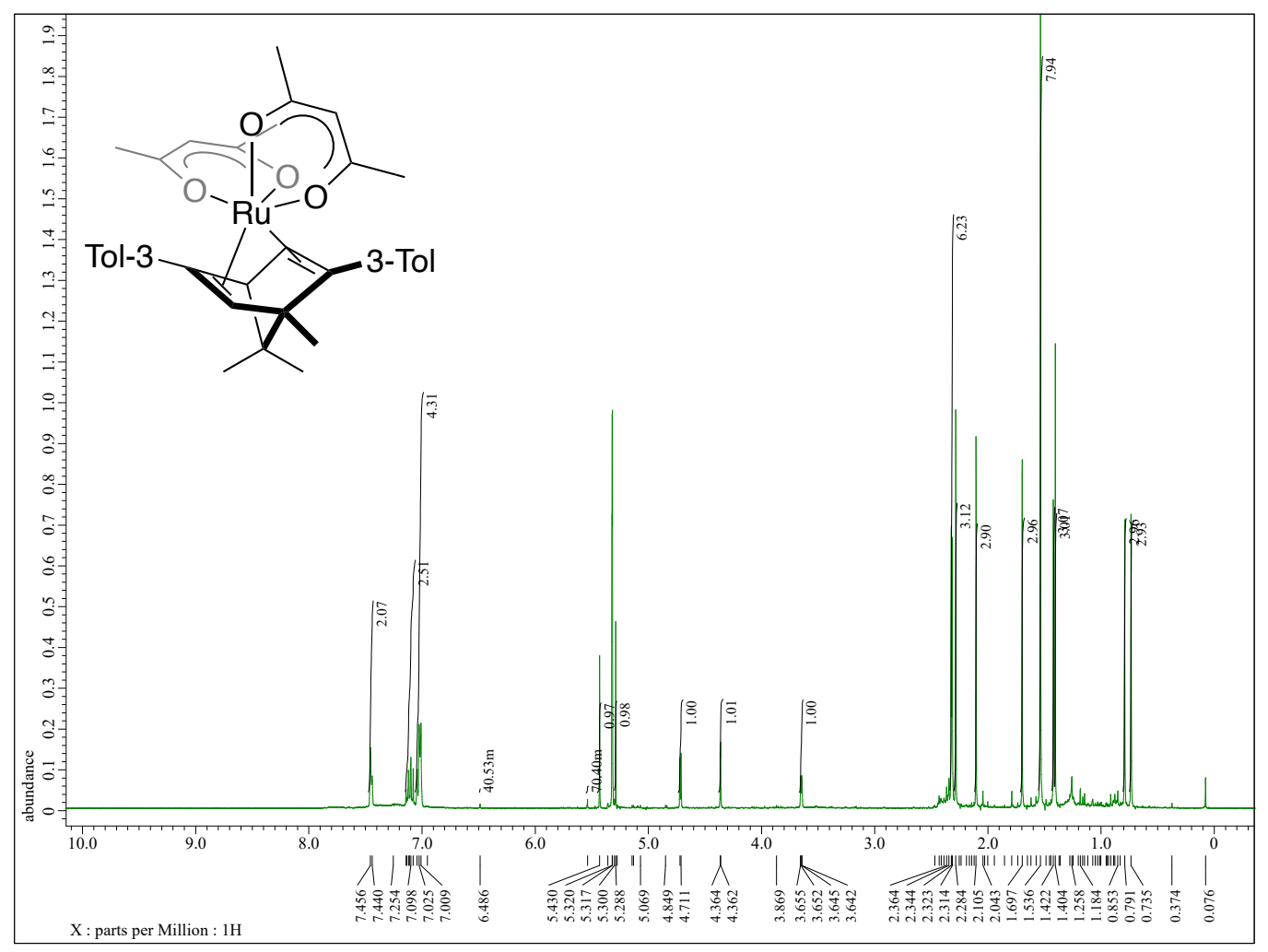

Figure S8-1. ' $\mathrm{H}$ NMR Spectrum of $\left[\mathrm{Ru}(\mathrm{acac})_{2}\left\{\eta^{4}-(1 \mathrm{~S}, 4 \mathrm{~S})-(3-\mathrm{Tol})_{2}\right.\right.$-bhd* $]$ (1e) $\left(400 \mathrm{MHz}, \mathrm{CD}_{2} \mathrm{Cl}_{2}\right.$, r.t.).

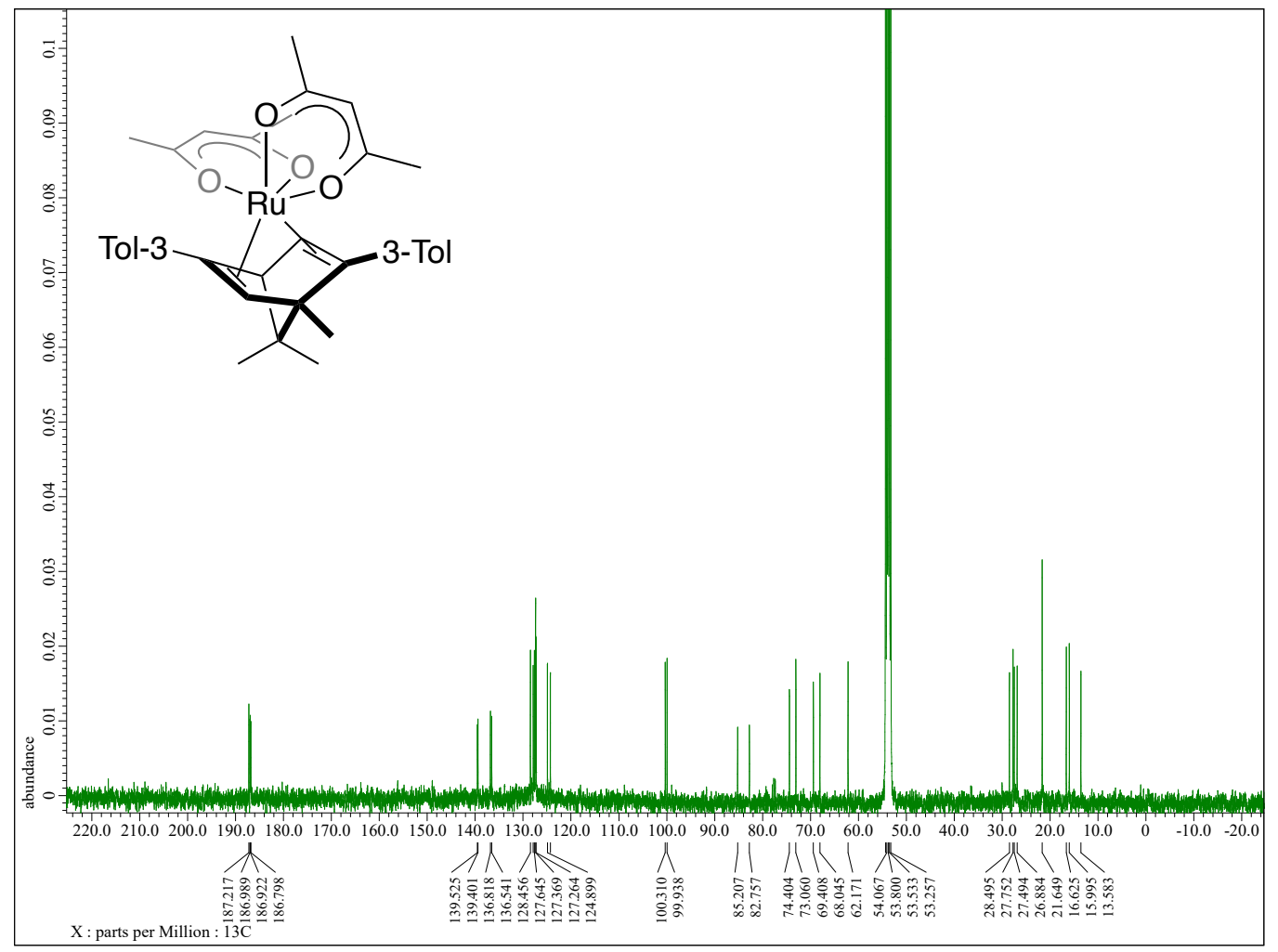

Figure S8-2. ${ }^{13} \mathrm{C}\left\{{ }^{1} \mathrm{H}\right\}$ NMR Spectrum of $\left[\mathrm{Ru}(\mathrm{acac}){ }_{2}\left\{\eta^{4}-(1 \mathrm{~S}, 4 S)-\left(3-\mathrm{Tol}_{2}\right.\right.\right.$-bhd $\left.{ }^{*}\right](\mathbf{1 e})\left(100 \mathrm{MHz}, \mathrm{CD}_{2} \mathrm{Cl}_{2}\right.$, r.t.). 


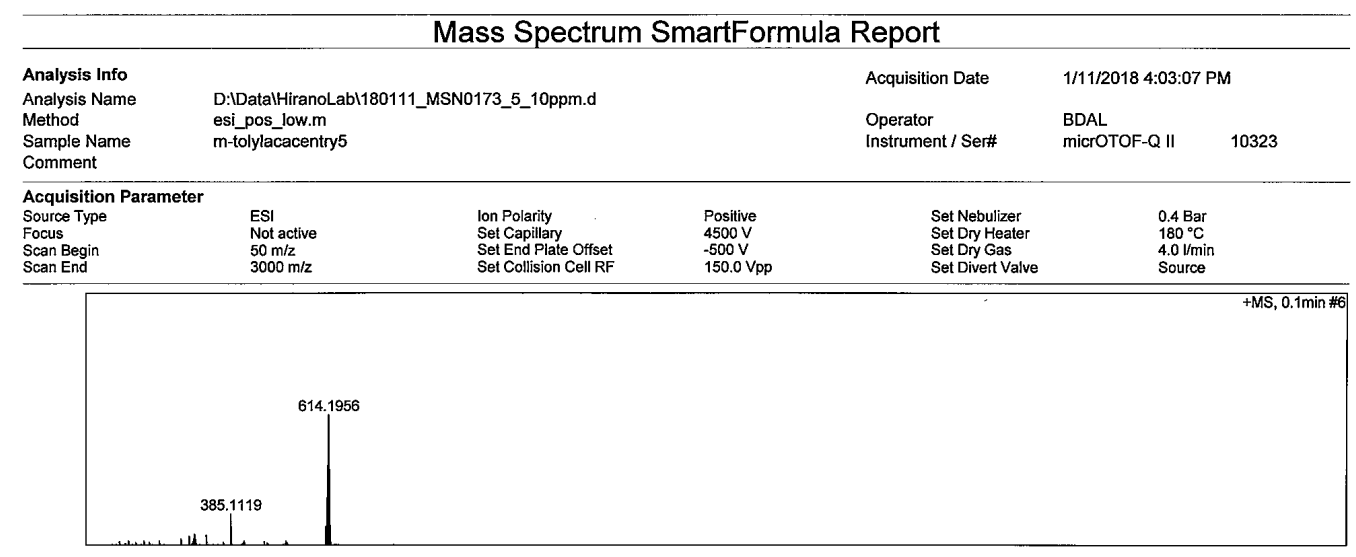

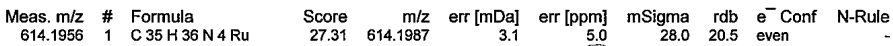

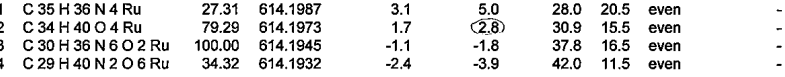

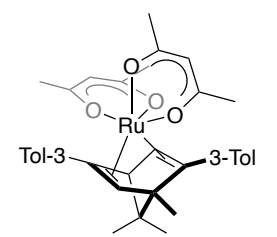

Figure S8-3. HRMS (ESI) Spectrum of $\left[\mathrm{Ru}(\mathrm{acac})_{2}\left\{\eta^{4}-(1 S, 4 S)-(3-\mathrm{Tol})_{2}-\mathrm{bhd}^{*}\right](\mathbf{1 e})\right.$.

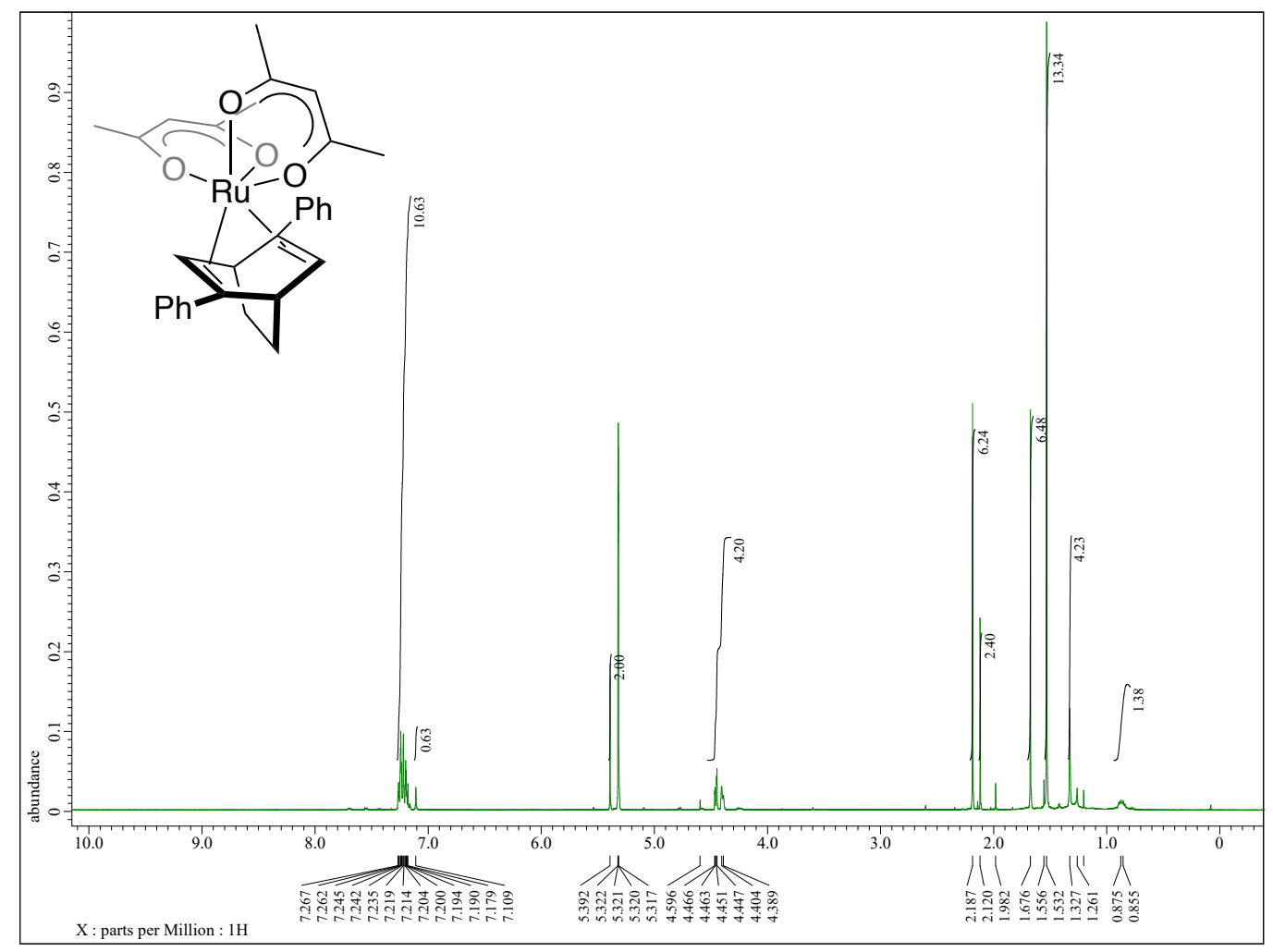

Figure S9-1. ${ }^{1} \mathrm{H}$ NMR of $\left[\mathrm{Ru}(\mathrm{acac})_{2}\left\{\eta^{4}-(1 \mathrm{~S}, 4 \mathrm{~S})-\mathrm{Ph}_{2}\right.\right.$-bod* $\left.\}\right]$ (1f). Major isomer $\left(400 \mathrm{MHz}, \mathrm{CD}_{2} \mathrm{Cl}_{2}\right.$, r.t.). 


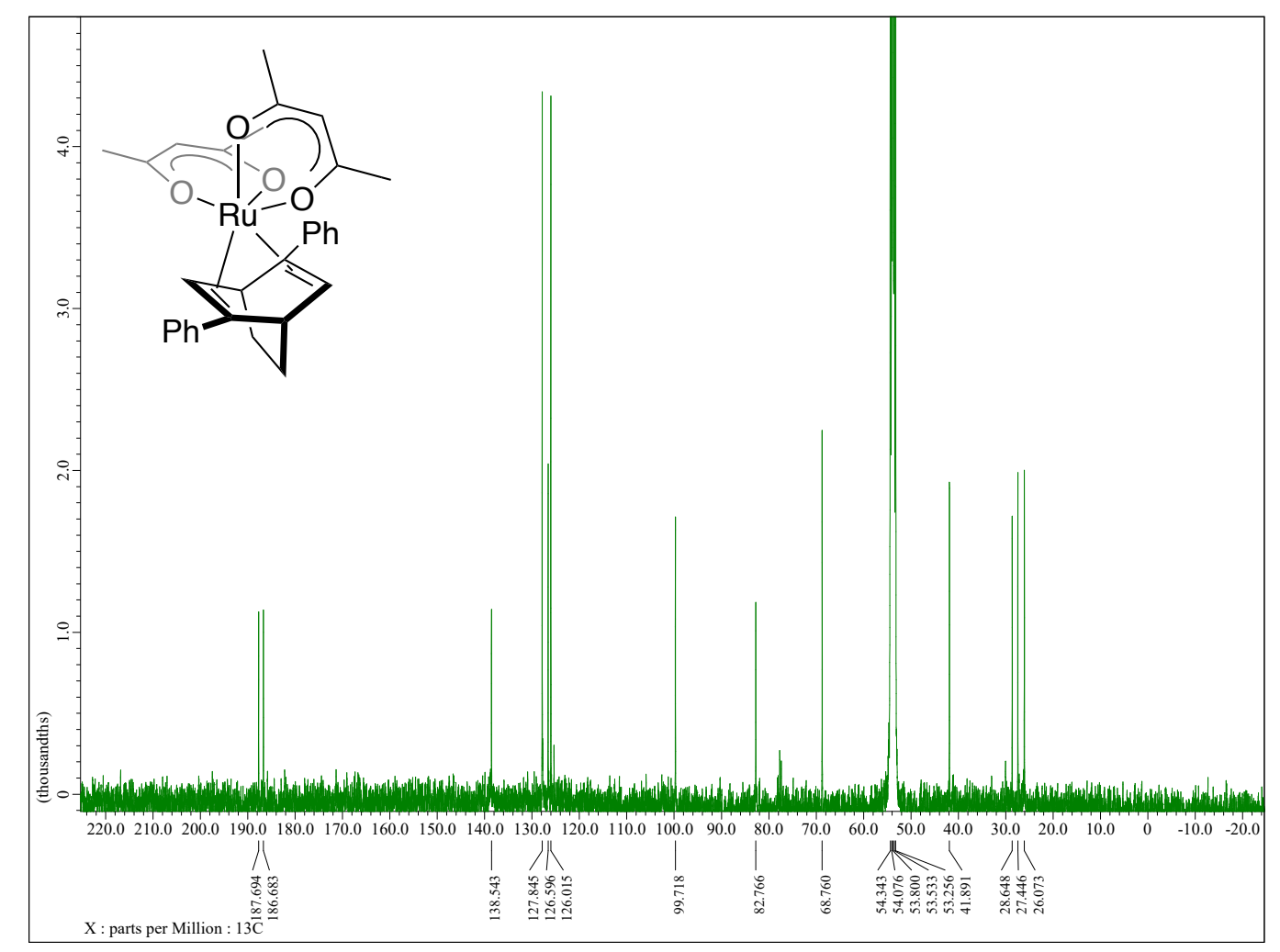

Figure S9-2. ${ }^{13} \mathrm{C}\left\{{ }^{1} \mathrm{H}\right\} \mathrm{NMR}$ of $\left[\mathrm{Ru}(\text { acac })_{2}\left\{\eta^{4}-(1 \mathrm{~S}, 4 \mathrm{~S})-\mathrm{Ph}_{2}-\right.\right.$ bod $\left.\left.^{*}\right\}\right]$ (1f). Major isomer $\left(100 \mathrm{MHz}, \mathrm{CD}_{2} \mathrm{Cl}_{2}\right.$, r.t.).

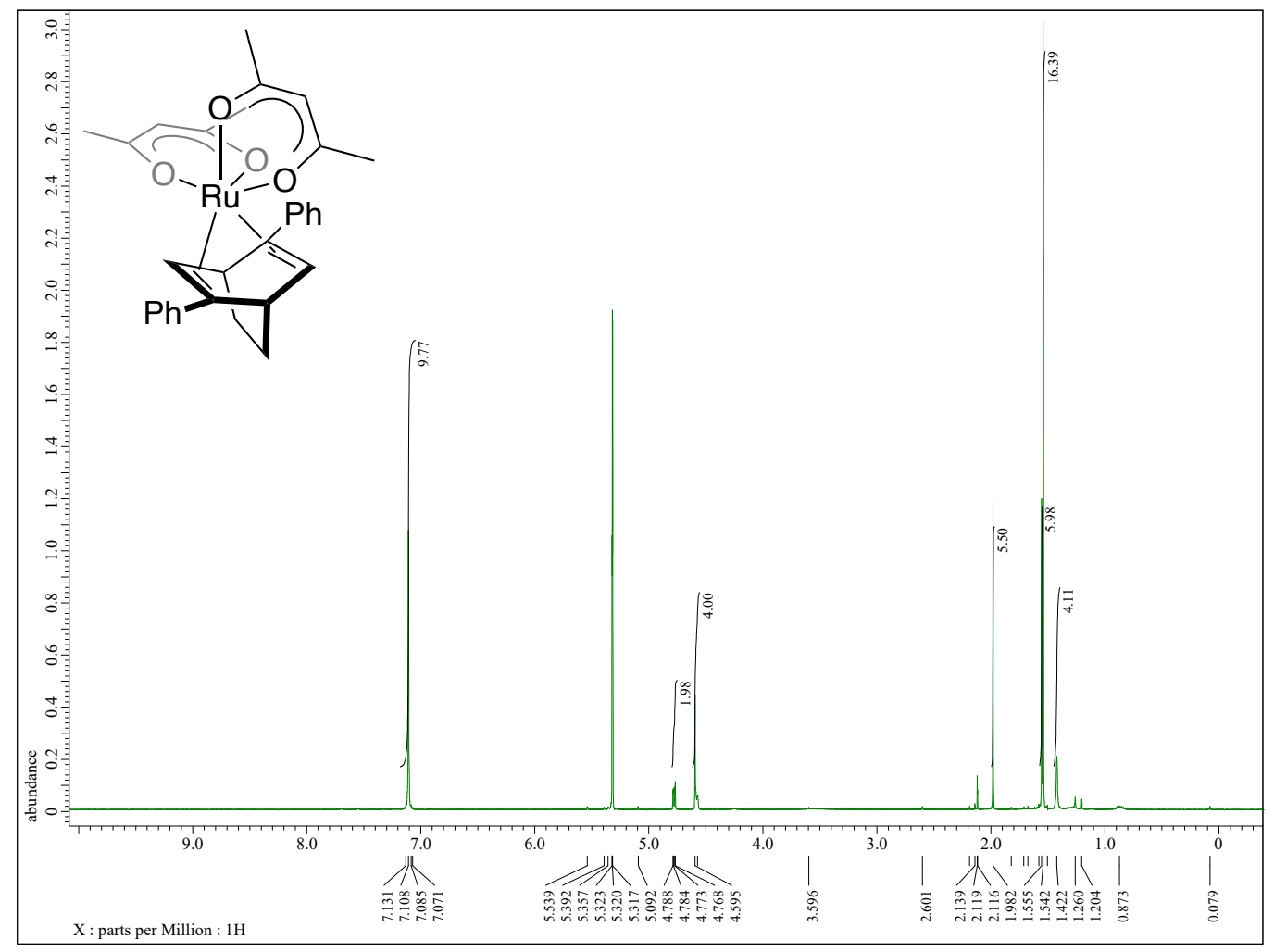

Figure S9-3. ${ }^{1} \mathrm{H}$ NMR of $\left[\mathrm{Ru}(\mathrm{acac})_{2}\left\{\eta^{4}-(1 \mathrm{~S}, 4 S)-\mathrm{Ph}_{2}\right.\right.$-bod $\left.\left.{ }^{*}\right\}\right]$ (1f). Minor isomer $\left(400 \mathrm{MHz}, \mathrm{CD}_{2} \mathrm{Cl}_{2}\right.$, r.t.) 


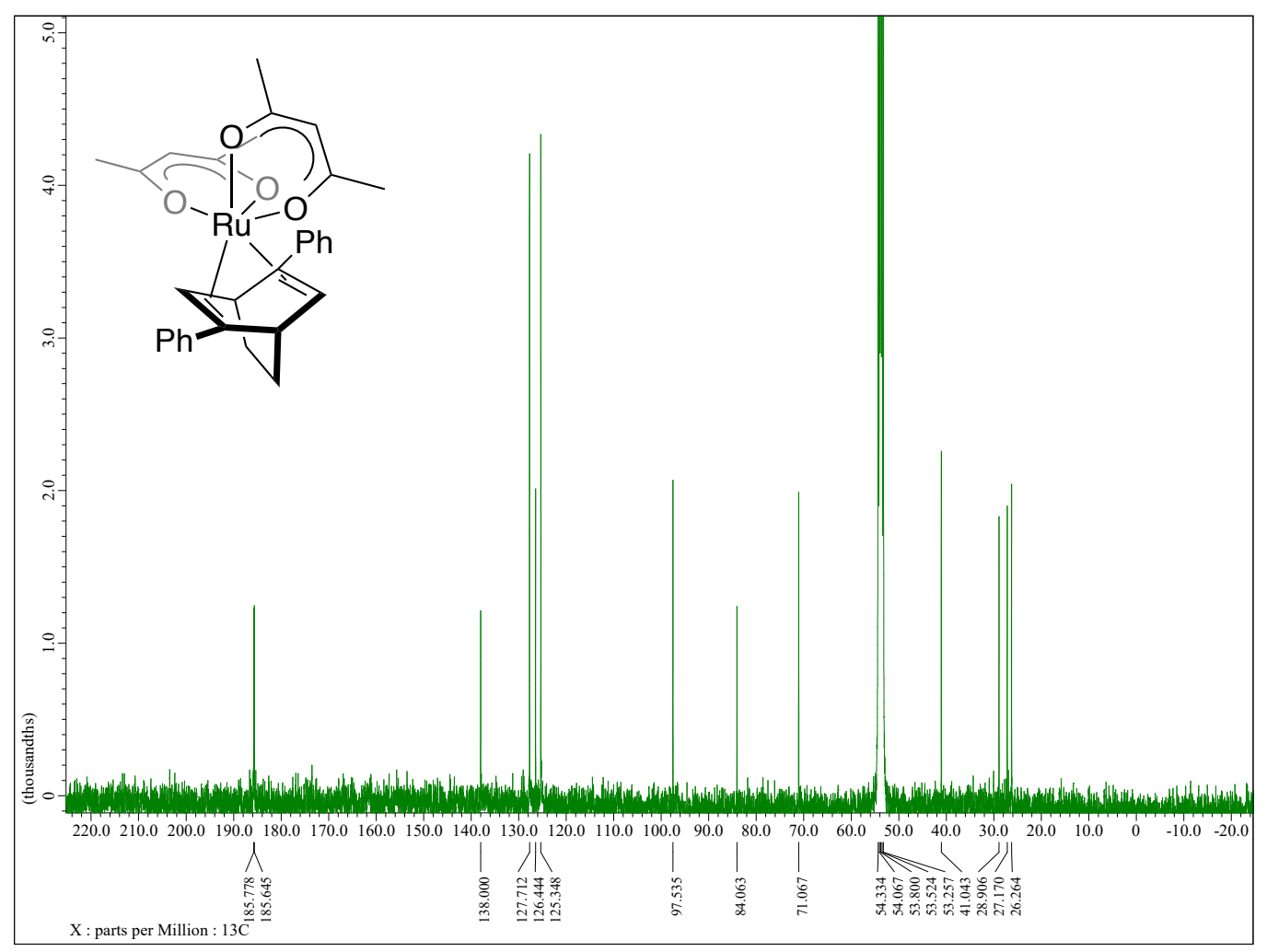

Figure S9-4. ${ }^{13} \mathrm{C}\left\{{ }^{1} \mathrm{H}\right\} \mathrm{NMR}$ of $\left[\mathrm{Ru}(\mathrm{acac})_{2}\left\{\eta^{4}-(1 \mathrm{~S}, 4 \mathrm{~S})-\mathrm{Ph}_{2}\right.\right.$-bod $\left.\left.{ }^{*}\right\}\right]$ (1f). Minor isomer $\left(100 \mathrm{MHz}, \mathrm{CD}_{2} \mathrm{Cl}_{2}\right.$, r.t.)

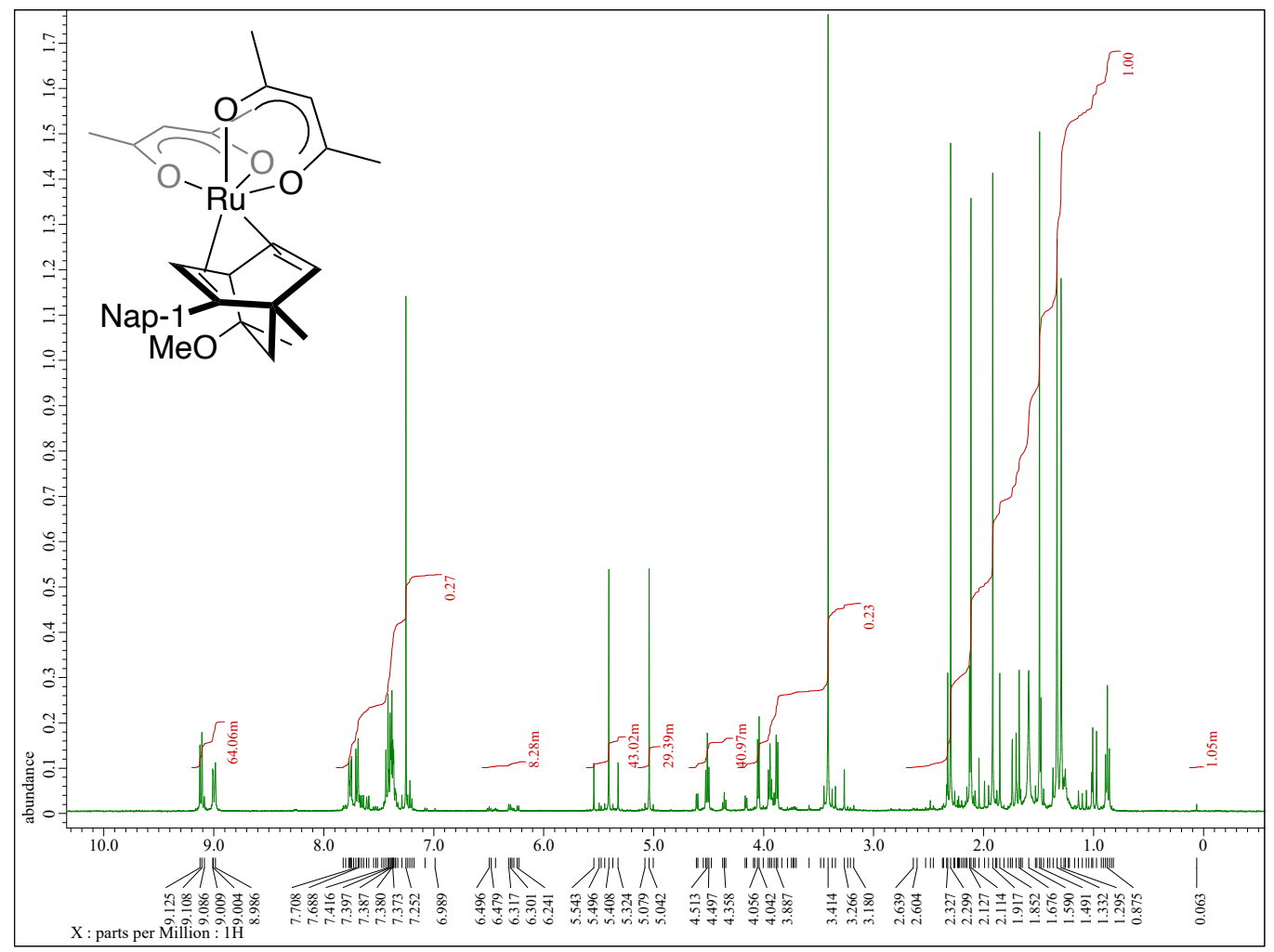

Figure S10-1. ${ }^{1} \mathrm{H}$ NMR Spectrum of $\left[\mathrm{Ru}(\mathrm{acac})_{2}\left\{\eta^{4}-(1 \mathrm{~S}, 4 R, 8 R)-(1-\mathrm{Nap})\right.\right.$-bod* $\left.\}\right]$ (1h) $\left(400 \mathrm{MHz}, \mathrm{CDCl}_{3}\right.$, r.t.). 


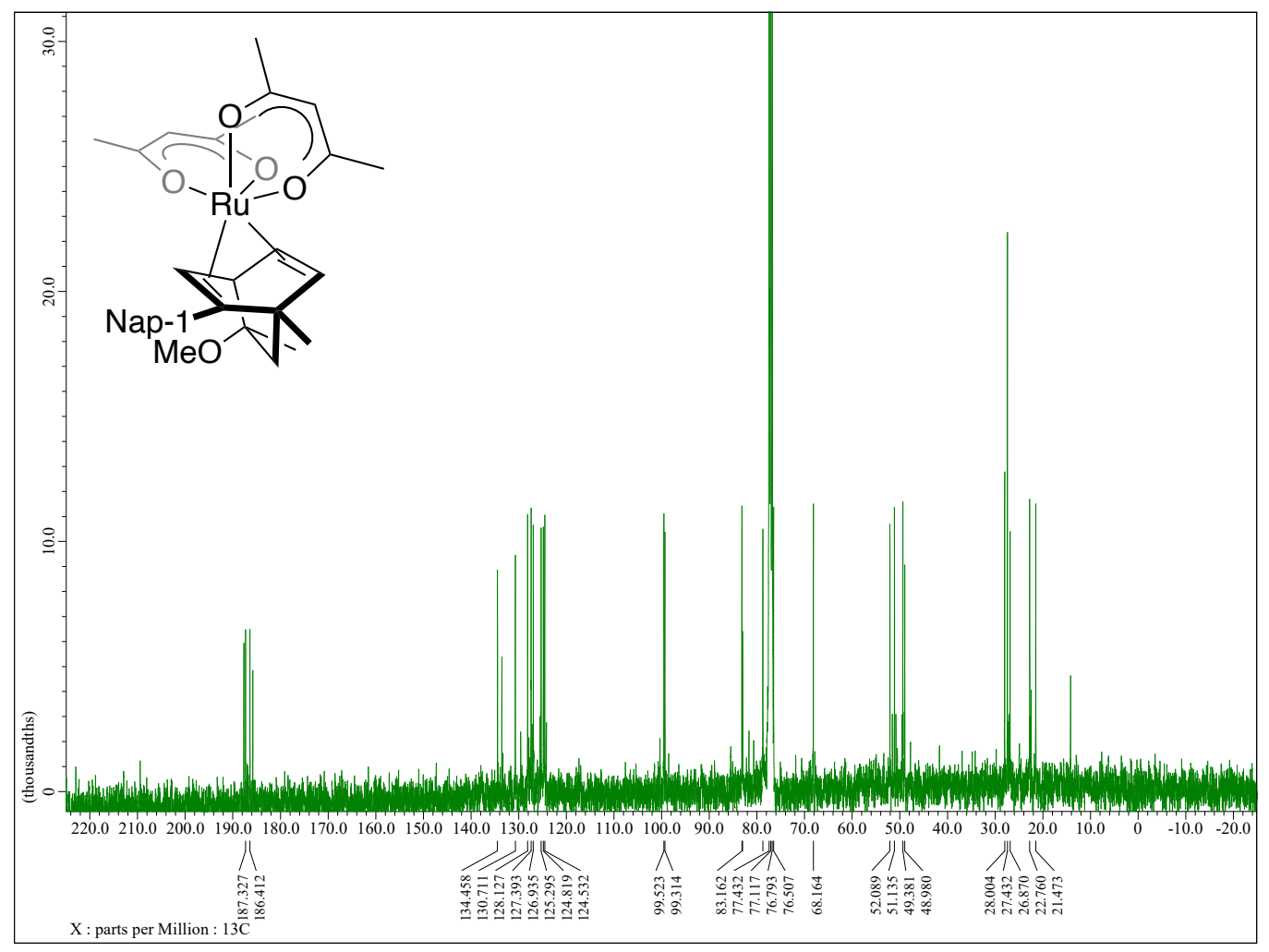

Figure S10-2. $\left.{ }^{13} \mathrm{C}^{\prime}{ }^{1} \mathrm{H}\right\}$ NMR Spectrum of $\left[\mathrm{Ru}(\mathrm{acac})_{2}\left\{\eta^{4}-(1 \mathrm{~S}, 4 R, 8 R)-(1-\mathrm{Nap})-\mathrm{bod}^{*}\right\}\right](\mathbf{1 h})\left(100 \mathrm{MHz}, \mathrm{CDCl}_{3}\right.$ r.t.).

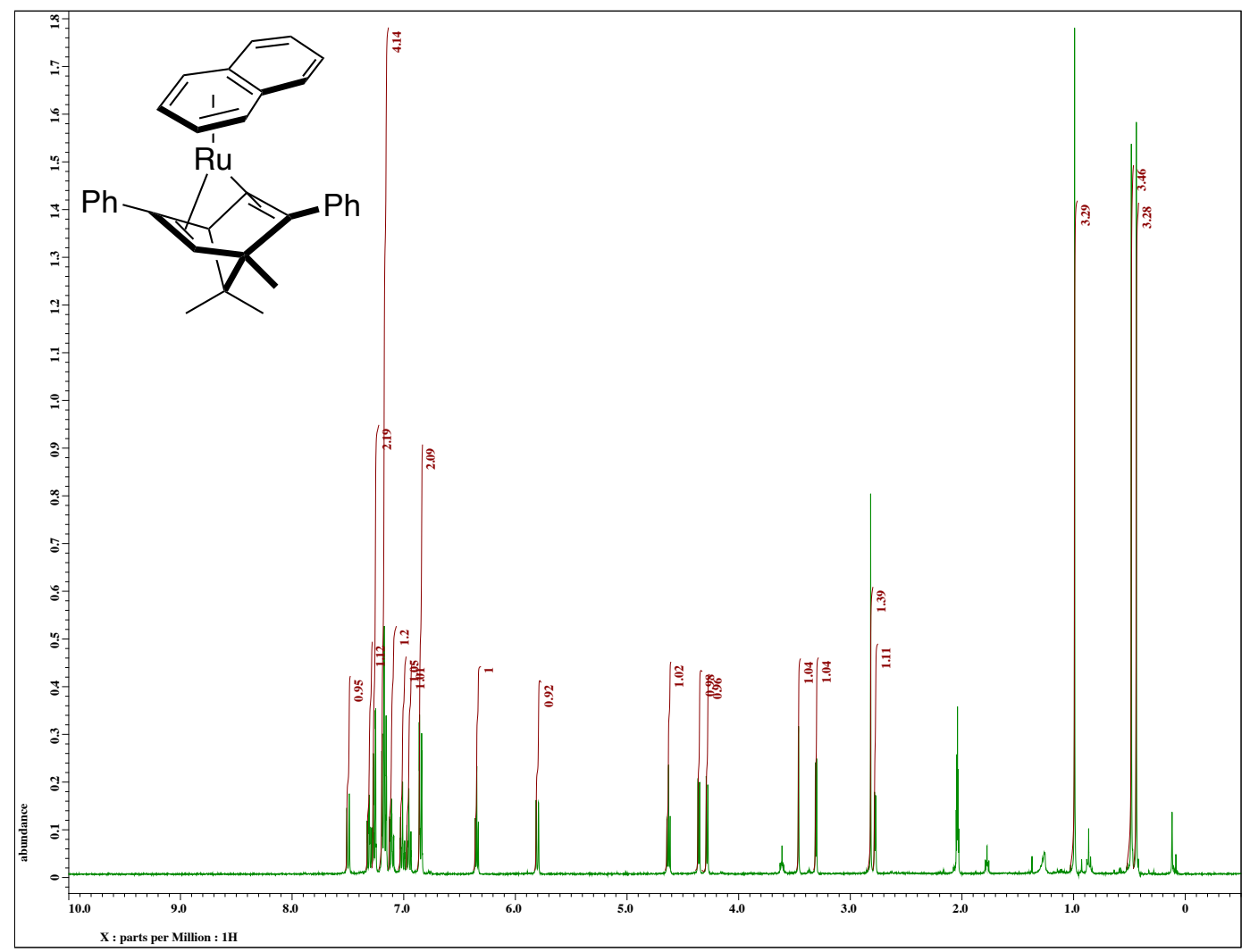

Figure S1 1-1. ${ }^{1} \mathrm{H}$ NMR Spectrum of $\left[\mathrm{Ru}\left(\eta^{6}\right.\right.$-naphthalene) $\left.\left\{\eta^{4}-(1 S, 4 S)-\mathrm{Ph}_{2}-\mathrm{bhd}^{*}\right\}\right](2 \mathrm{a})(400 \mathrm{MHz}$, acetone$d_{6}$, r.t.). 


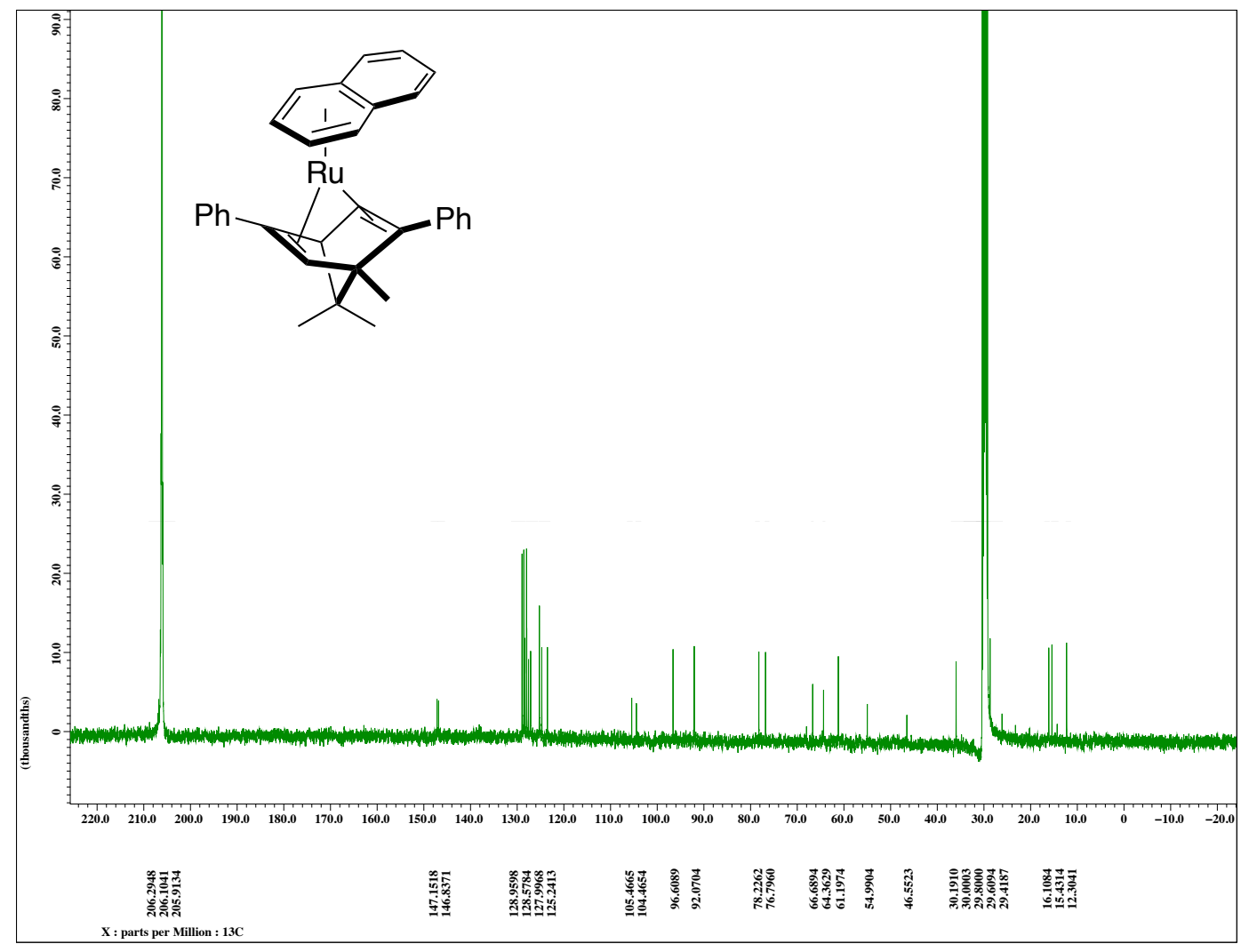

Figure S11-2. ${ }^{13} \mathrm{C}\left\{{ }^{1} \mathrm{H}\right\} \mathrm{NMR}$ Spectrum of $\left[\mathrm{Ru}\left(\eta^{6}\right.\right.$-naphthalene) $\left.\left\{\eta^{4}-(1 \mathrm{~S}, 4 S)-\mathrm{Ph}_{2}-\mathrm{bhd} \mathrm{d}^{*}\right\}\right](\mathbf{2 a})(100 \mathrm{MHz}$, acetone- $d_{6}$, r.t.).

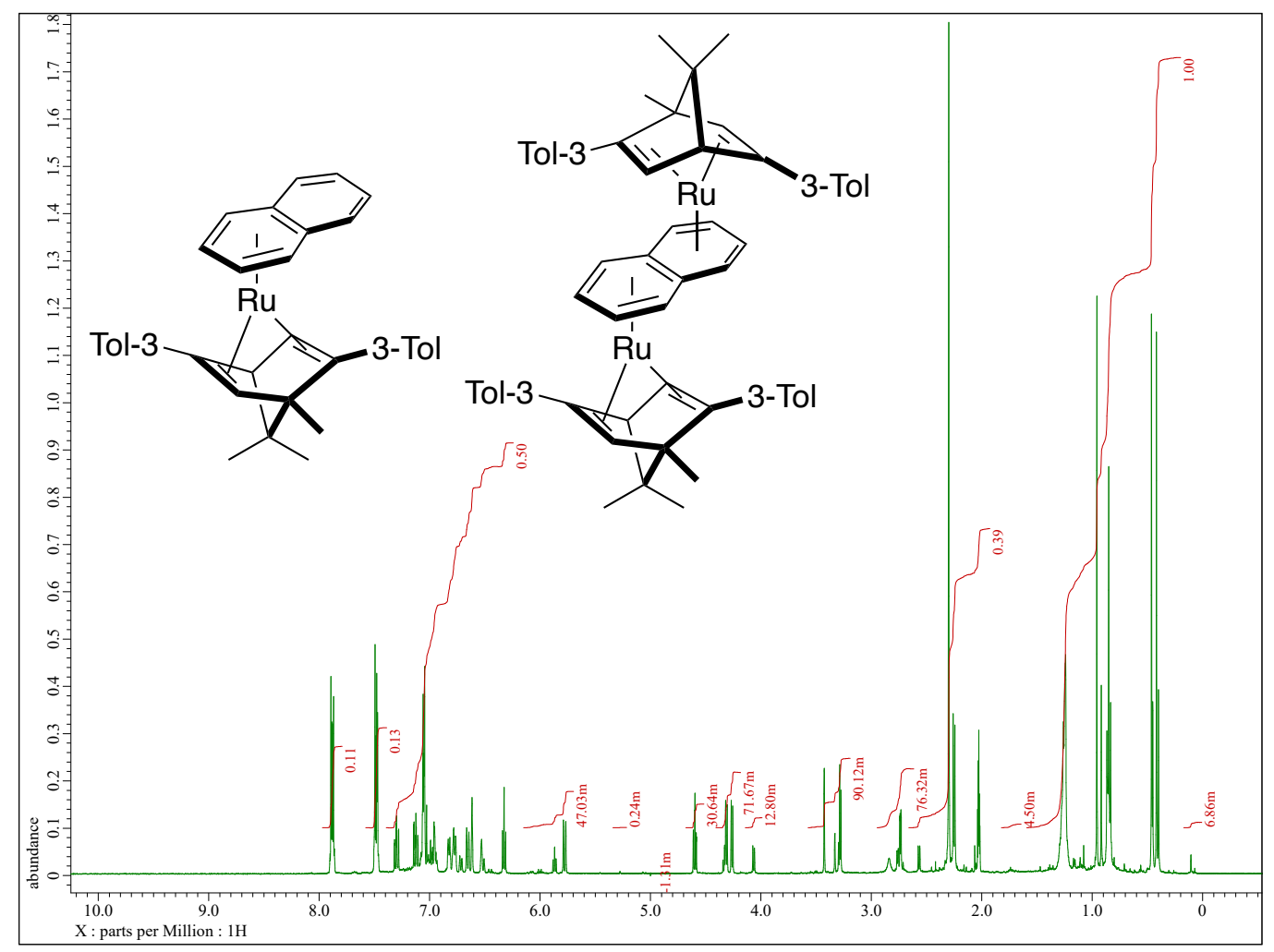

Figure S12-1. ${ }^{1} \mathrm{H}$ NMR Spectrum of $\left[\mathrm{Ru}\left(\eta^{6}\right.\right.$-naphthalene) $\left.\left\{\eta^{4}-(1 S, 4 S)-(3-\mathrm{Tol})_{2}-\mathrm{bhd}^{*}\right\}\right](\mathbf{2 e})(400 \mathrm{MHz}$, acetone- $d_{6}$, r.t.). 


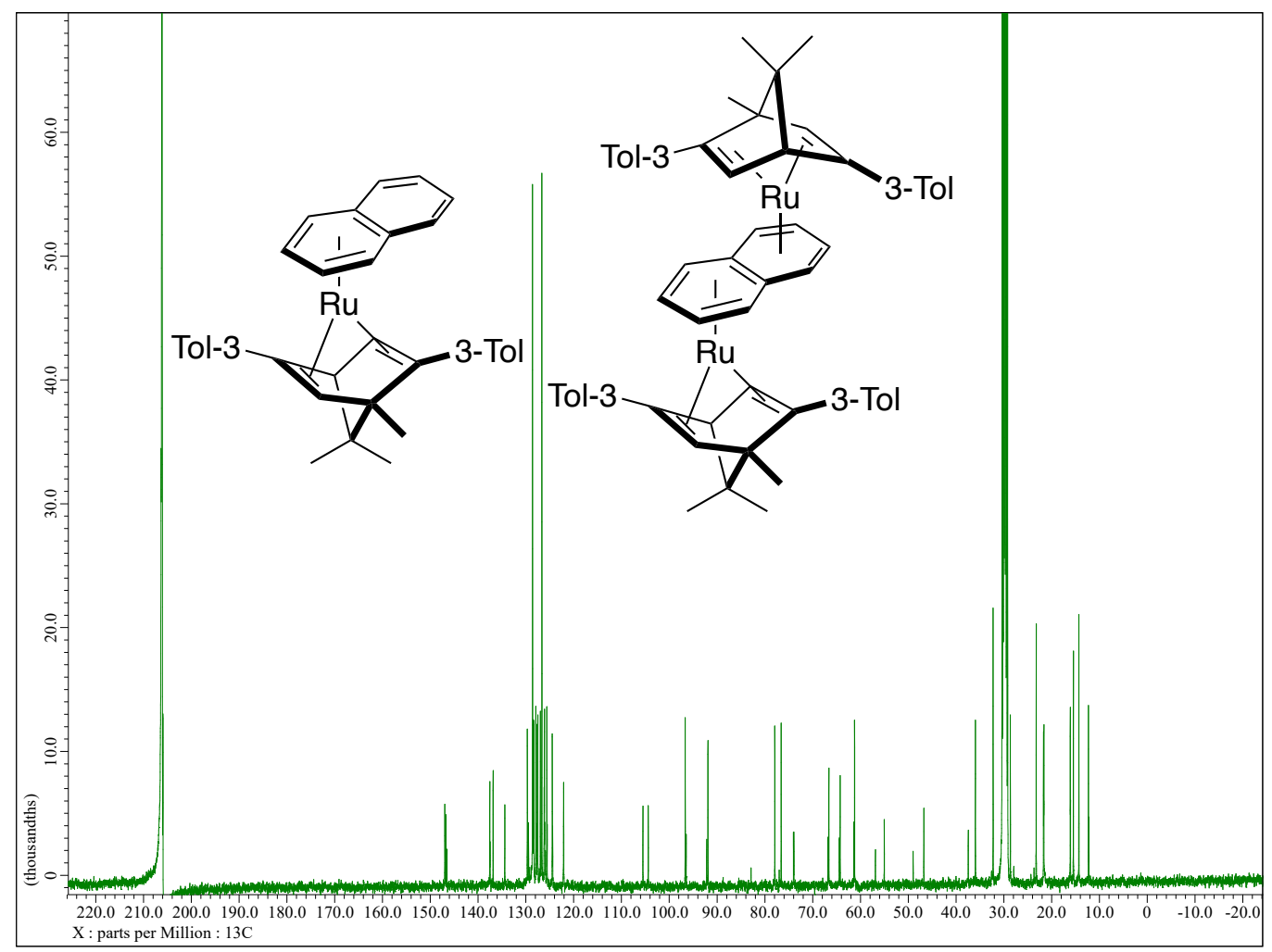

Figure S12-2. ${ }^{13} \mathrm{C}\left\{{ }^{1} \mathrm{H}\right\}$ NMR Spectrum of $\left[\mathrm{Ru}\left(\eta^{6}\right.\right.$-naphthalene $)\left\{\eta^{4}-(1 S, 4 S)-(3-\mathrm{Tol}){ }_{2}\right.$-bhd $\left.\left.\mathbf{d}^{*}\right\}\right](\mathbf{2 e})(100 \mathrm{MHz}$, acetone- $d_{6}$, r.t.).

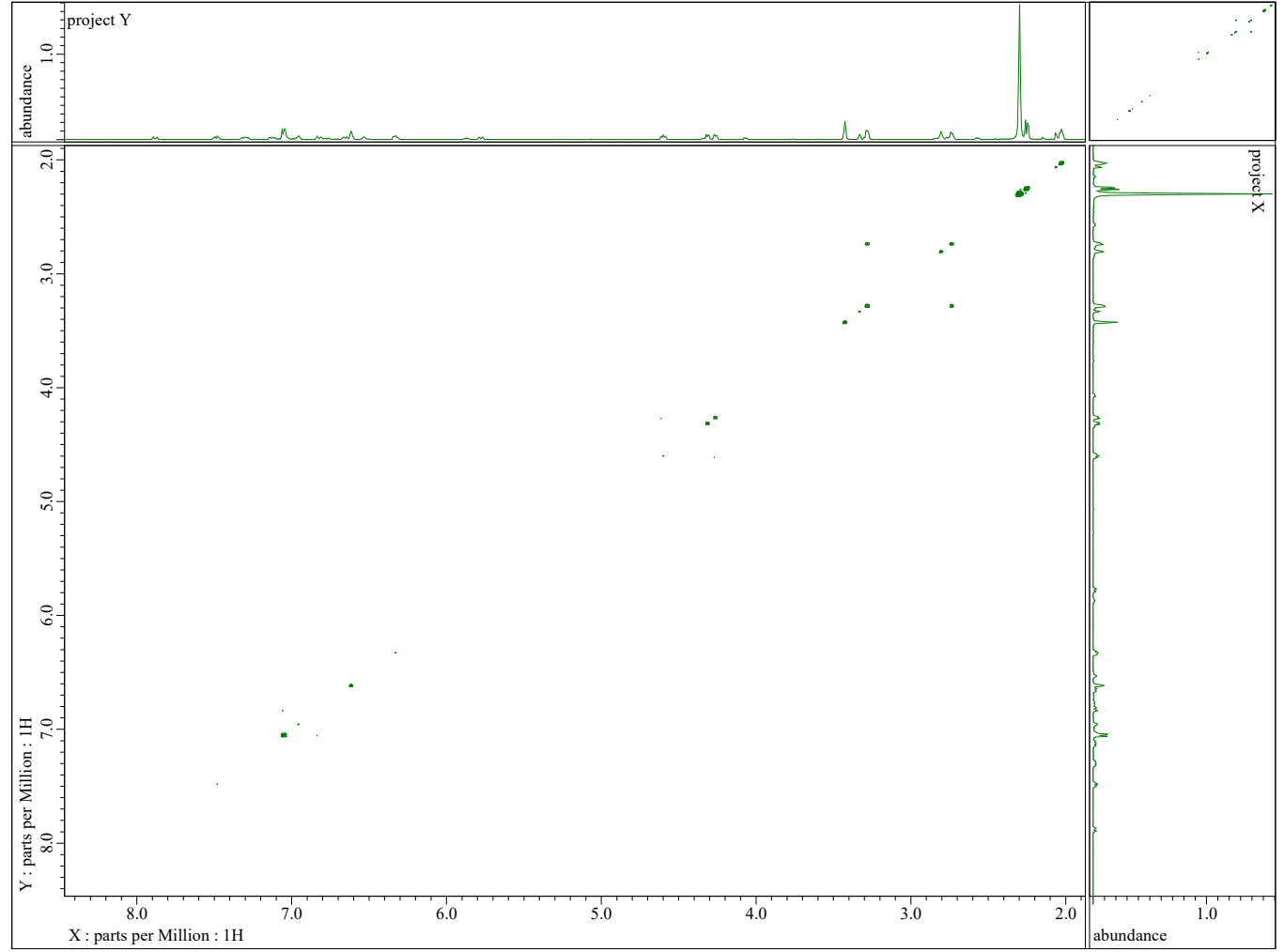

Figure S12-3. ${ }^{1} \mathrm{H}-{ }^{1} \mathrm{H}$ COSY Spectrum of $\left[\mathrm{Ru}\left(\eta^{6}\right.\right.$-naphthalene) $\left.\left\{\eta^{4}-(1 S, 4 S)-(3-\mathrm{Tol})_{2}-\mathrm{bhd}^{*}\right\}\right](\mathbf{2 e})(400 \mathrm{MHz}$, acetone- $d_{6}$, r.t.). 


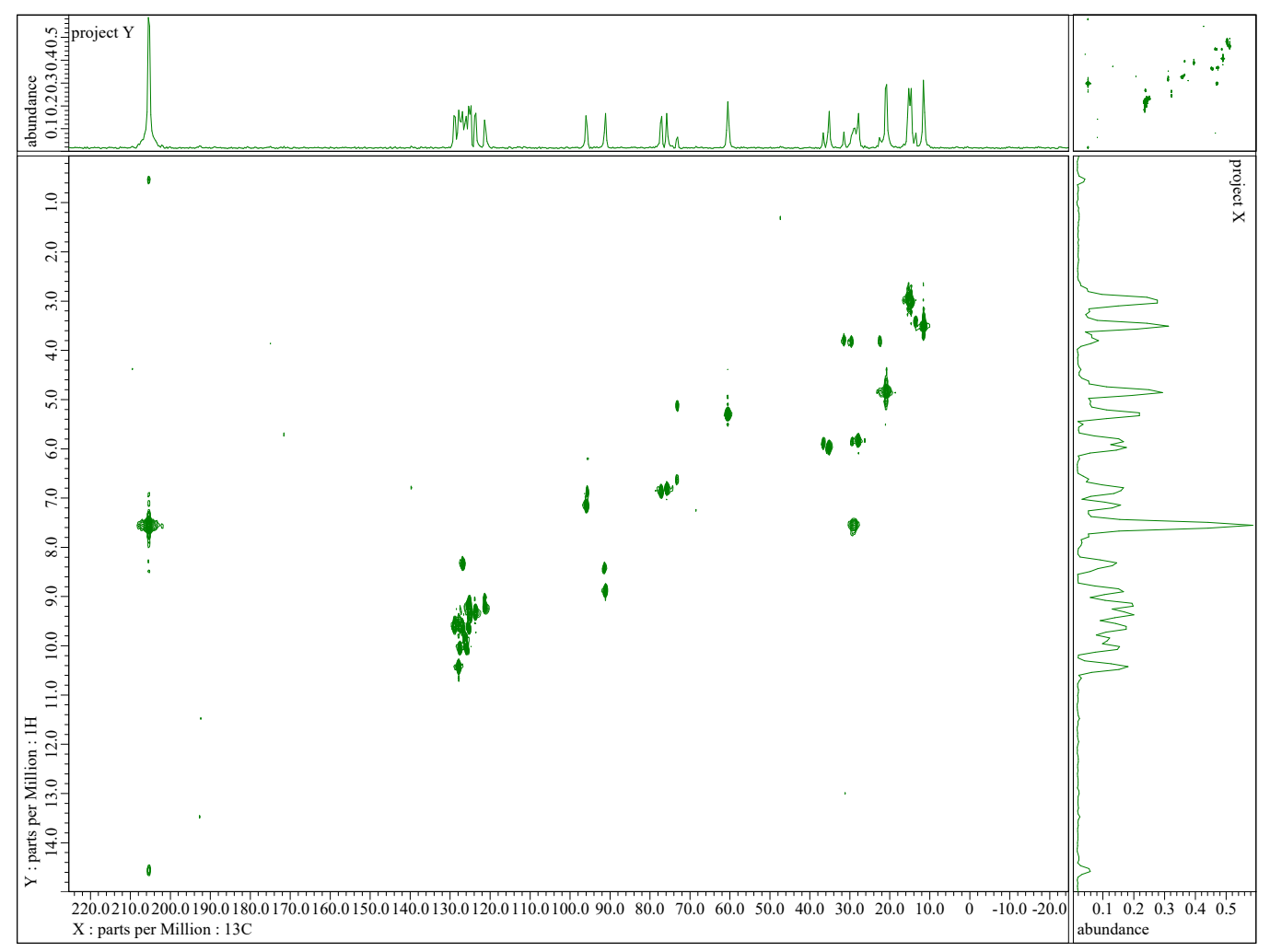

Figure S12-4. ${ }^{1} \mathrm{H}^{-13} \mathrm{C}$ HETCOR Spectrum of $\left[\mathrm{Ru}\left(\eta^{6}\right.\right.$-naphthalene $)\left\{\eta^{4}-(1 \mathrm{~S}, 4 S)-(3-\mathrm{Tol})_{2}\right.$-bhd* $\left.\}\right](\mathbf{2 e})(400 \mathrm{MHz}$, acetone- $d_{6}$, r.t.).

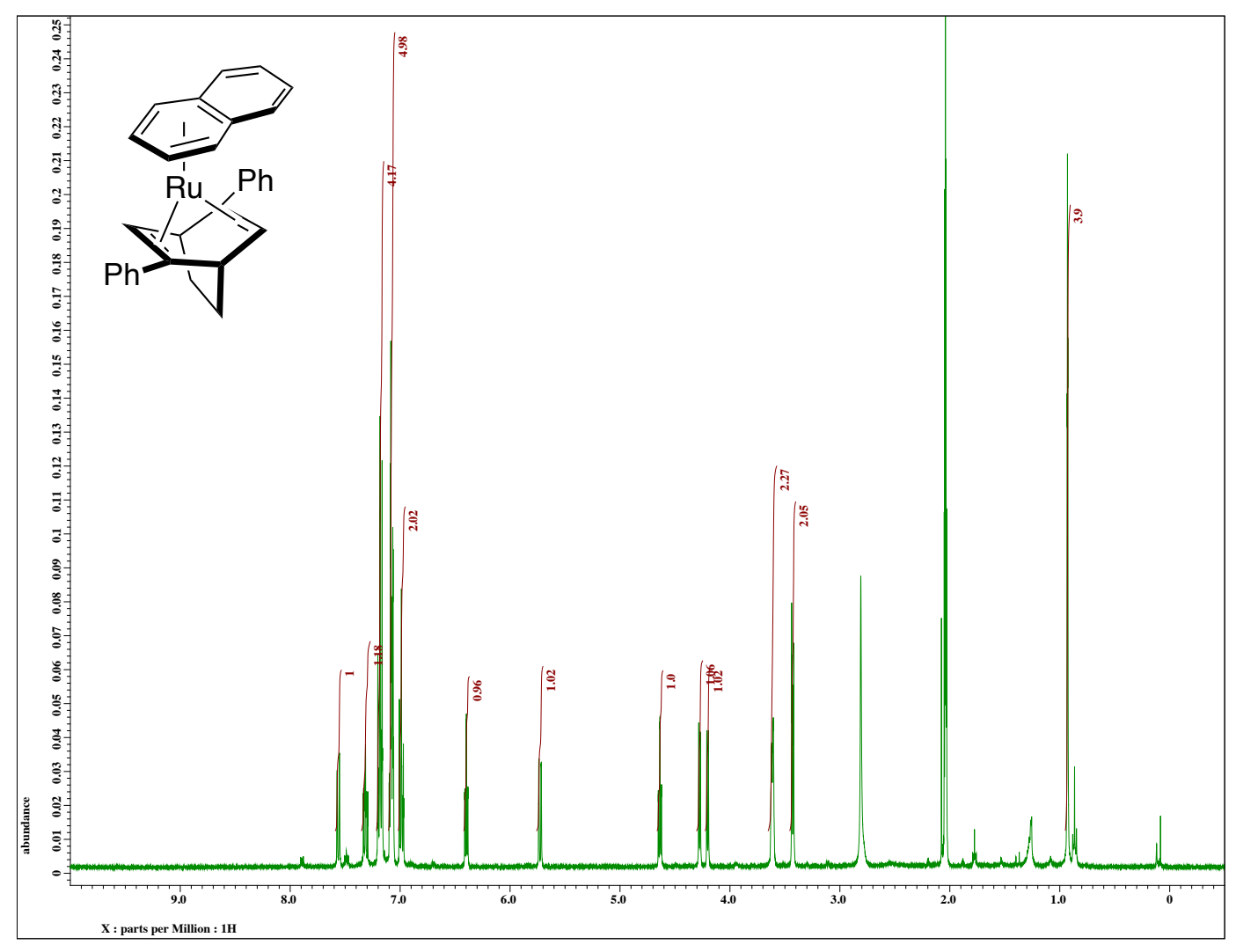

Figure S13-1. ${ }^{1} \mathrm{H}$ NMR Spectrum of $\left[\mathrm{Ru}\left(\eta^{6}\right.\right.$-naphthalene) $\left\{\eta^{4}-(1 S, 4 S)-\mathrm{Ph}_{2}\right.$-bod* $\left.\}\right](\mathbf{2 f})(400 \mathrm{MHz}$, acetone$d_{6}$, r.t.). 


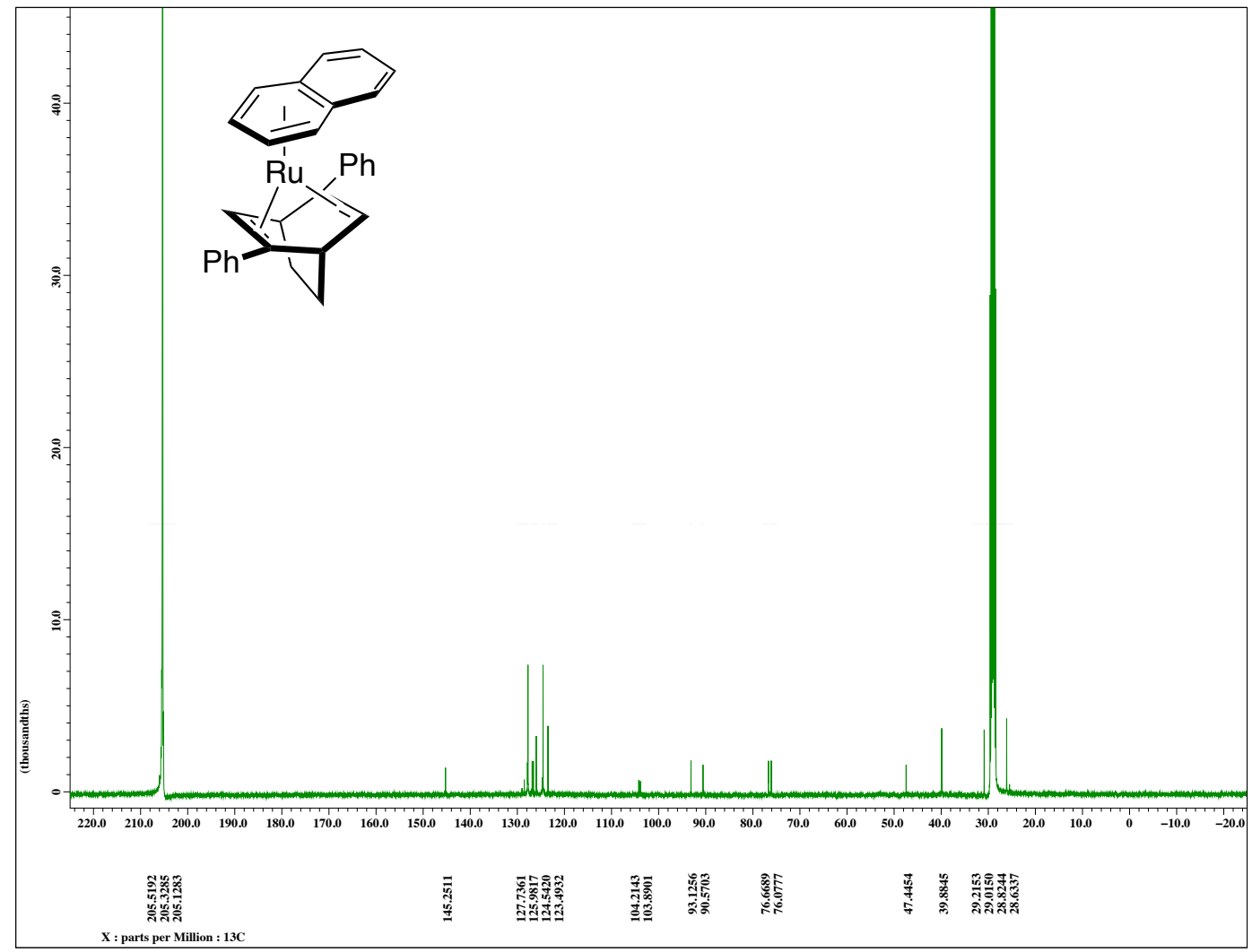

Figure S13-2. ${ }^{13} \mathrm{C}\{1 \mathrm{H}\}$ NMR Spectrum of $\left[\mathrm{Ru}\left(\eta^{6}\right.\right.$-naphthalene $)\left\{\eta^{4}-(1 S, 4 S)-\mathrm{Ph}_{2}-\right.$ bod $\left.\left.^{*}\right\}\right](\mathbf{2 f})(100 \mathrm{MHz}$, acetone- $d_{6}$, r.t.).

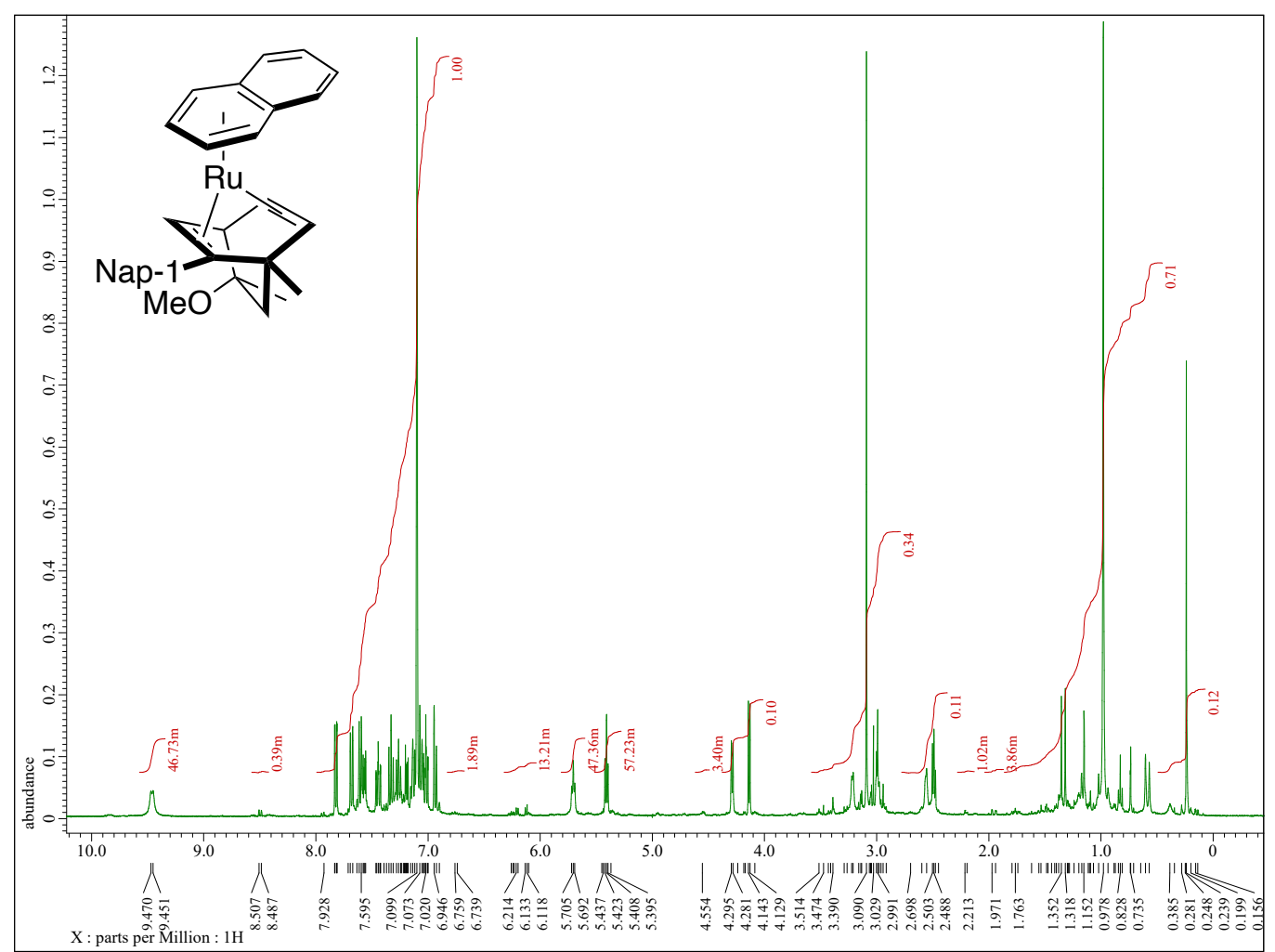

Figure S14-1. ${ }^{1} \mathrm{H}$ NMR Spectrum of $\left[\mathrm{Ru}\left(\eta^{6}\right.\right.$-naphthalene) $\left.\left\{\eta^{4}-(1 S, 4 R, 8 R)-(1-\mathrm{Nap})-\operatorname{bod}^{*}\right\}\right](\mathbf{2 h})(400 \mathrm{MHz}$, $\mathrm{C}_{6} \mathrm{D}_{6}$, r.t.). 


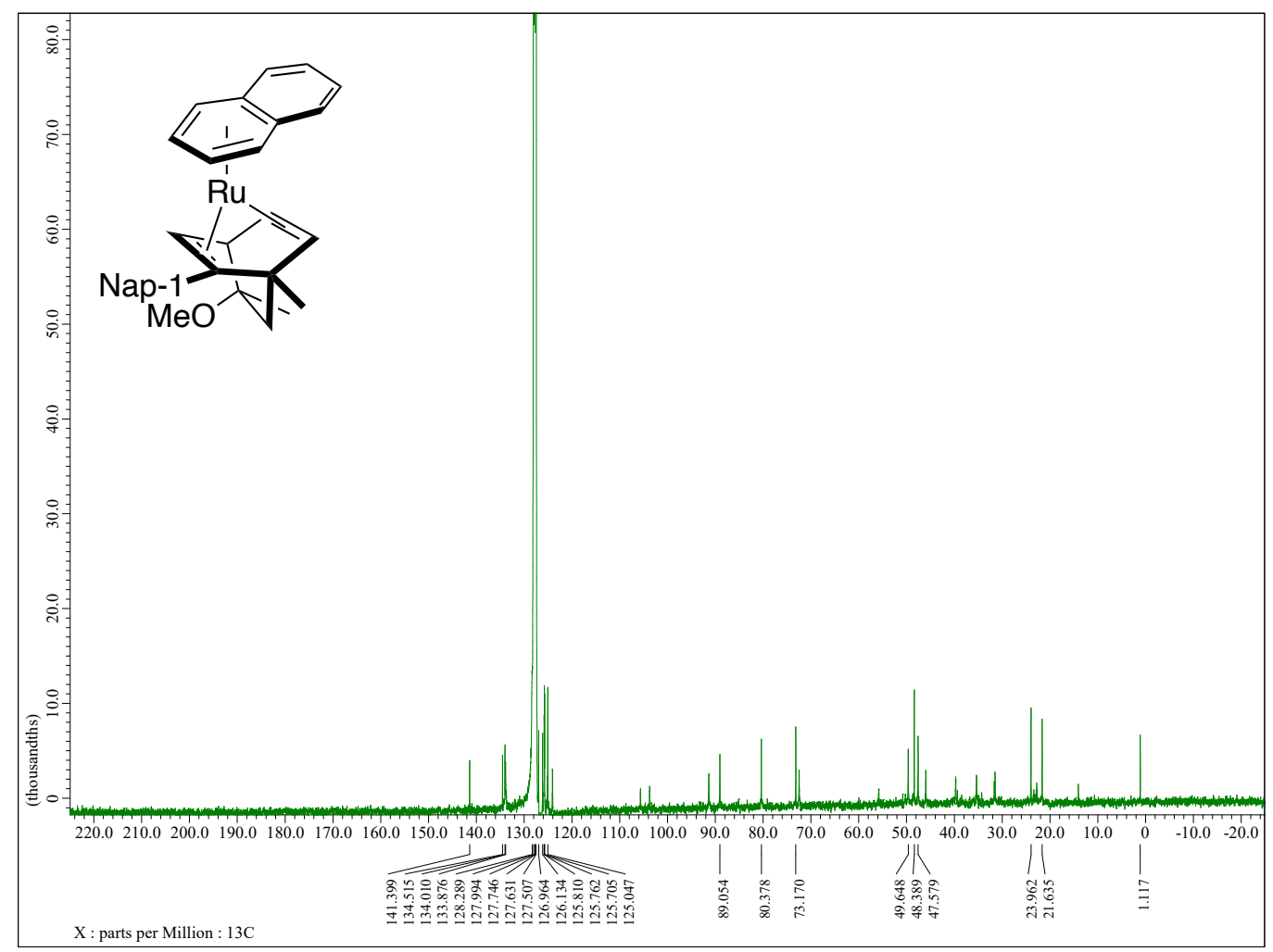

Figure S14-2. ${ }^{13} \mathrm{C}\left\{{ }^{1} \mathrm{H}\right\}$ NMR Spectrum of $\left[\mathrm{Ru}\left(\eta^{6}\right.\right.$-naphthalene) $\left\{\eta^{4}-(1 \mathrm{~S}, 4 R, 8 R)-(1-\mathrm{Nap})-\right.$ bod $\left.\left.{ }^{*}\right\}\right](\mathbf{2 h})(100$ $\mathrm{MHz}, \mathrm{C}_{6} \mathrm{D}_{6}$, r.t.).

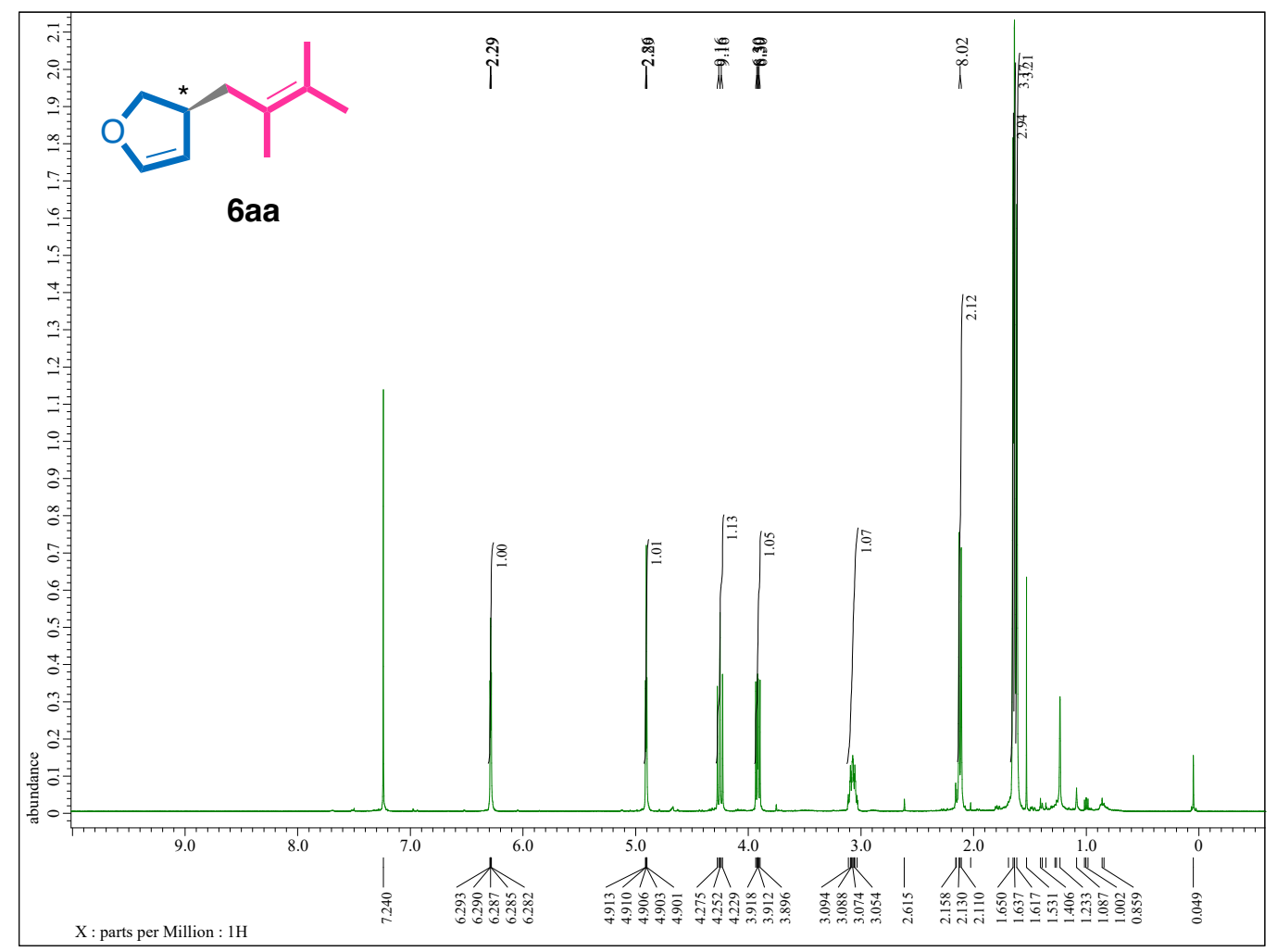

Figure S15-1. 'H NMR Spectrum of 3-(2,3-dimethylbut-2-en-1-yl)-2,3-dihydrofuran (6aa) $(400 \mathrm{MHz}$, $\mathrm{CDCl}_{3}$, r.t.). 


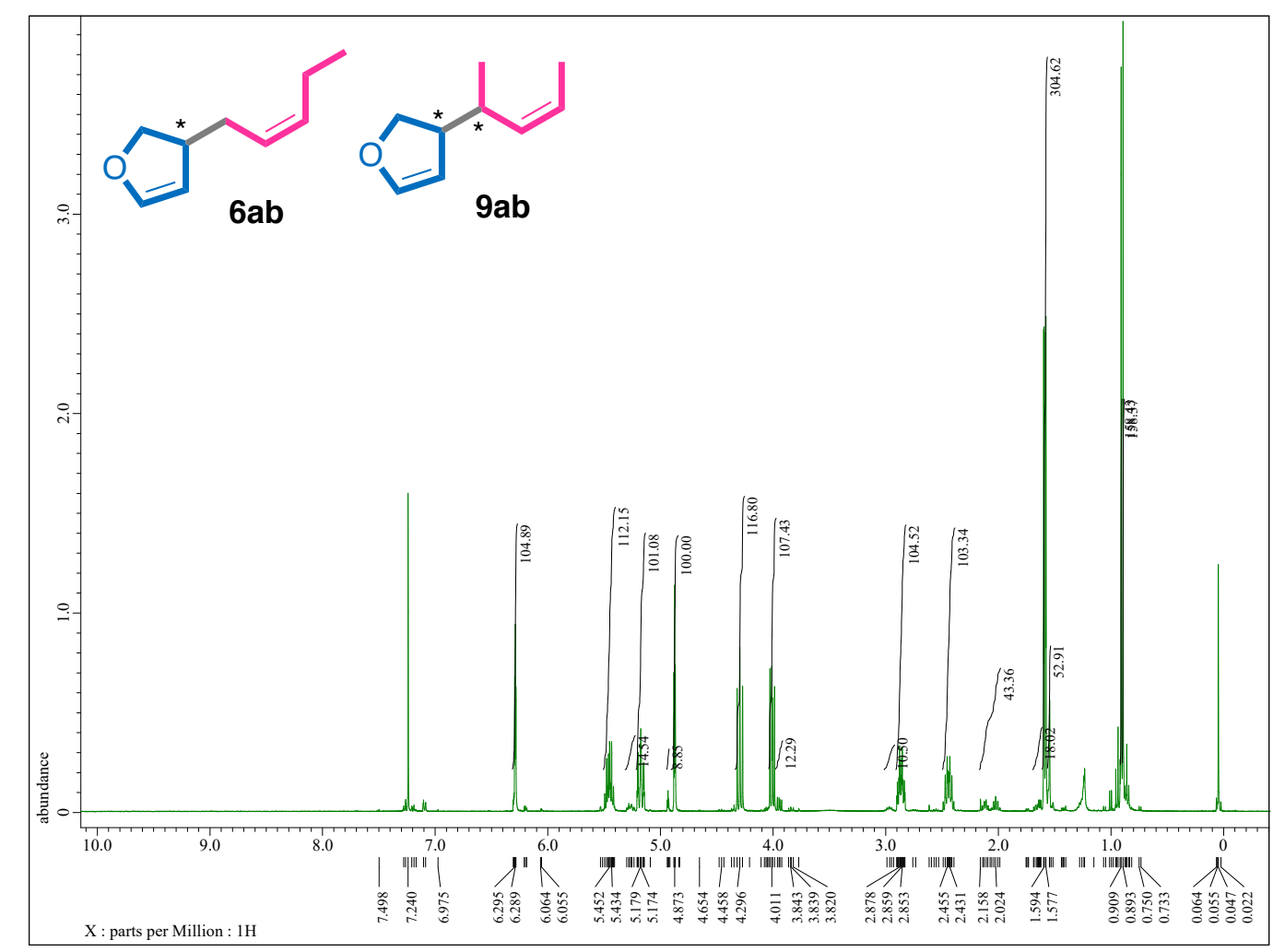

Figure S16-1. 'H NMR Spectrum of (Z)-3-(pen-2-en-1-yl)-2,3-dihydrofuran (6ab) and (Z)-3-(pen-2-en-1yl)-2,3-dihydrofuran (9ab) $\left(400 \mathrm{MHz}, \mathrm{CDCl}_{3}\right.$, r.t.).

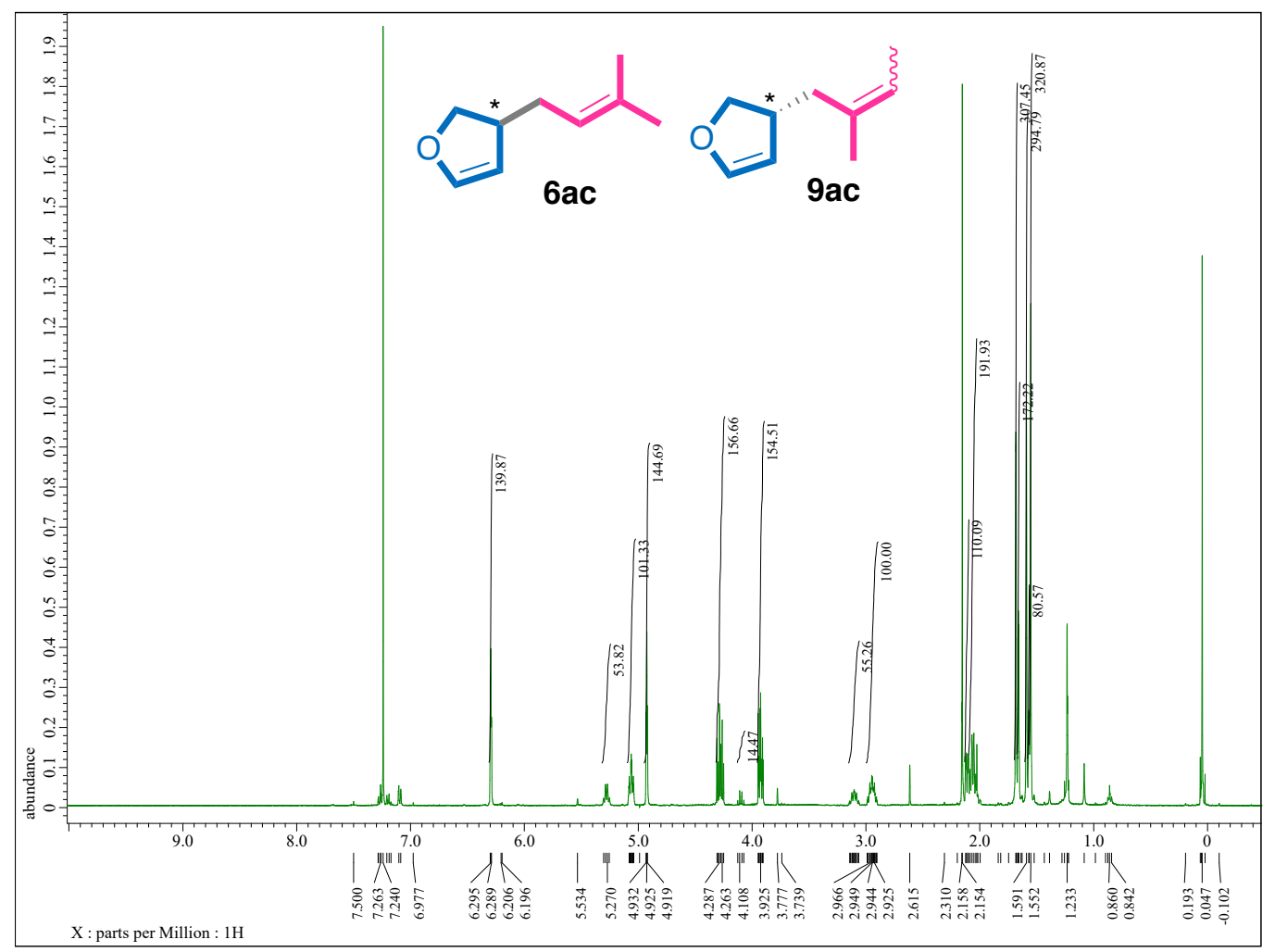

Figure S17-1. ' $\mathrm{H}$ NMR Spectrum of (Z)-3-(3-methylbut-2-en-1-yl)-2,3-dihydrofuran (6ac) and (Z)-3-(2methylbut-2-en-1-yl)-2,3-dihydrofuran (9ac) $\left(400 \mathrm{MHz}, \mathrm{CDCl}_{3}\right.$, r.t.). 


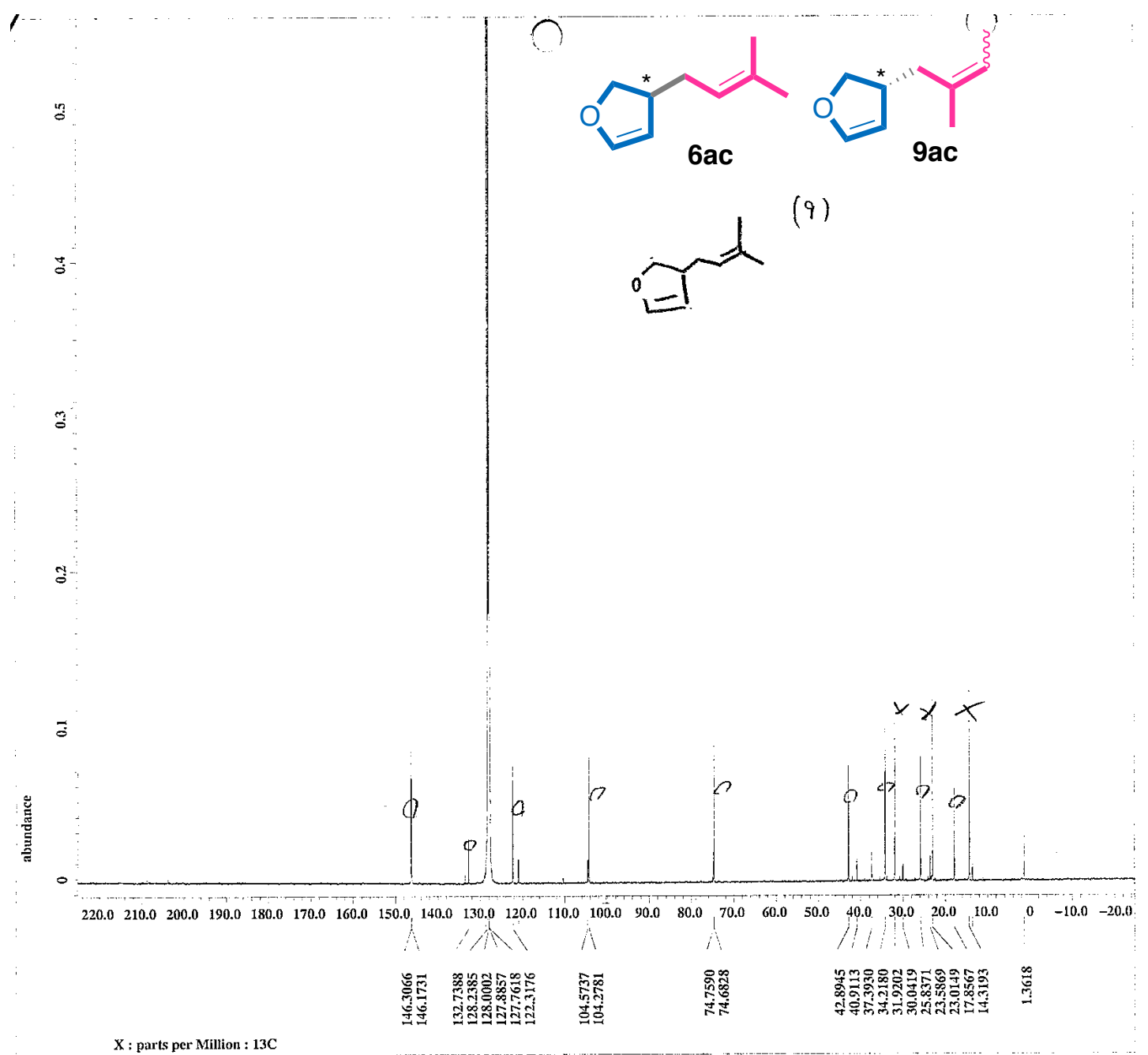

Figure S17-2. ${ }^{13} \mathrm{C}\left\{{ }^{1} \mathrm{H}\right\}$ NMR Spectrum of 3-(3-methylbut-2-en-1-yl)-2,3-dihydrofuran (6ac) and 3-(2methylbut-2-en-1-yl)-2,3-dihydrofuran (9ac) $\left(100 \mathrm{MHz}, \mathrm{C}_{6} \mathrm{D}_{6}\right.$, r.t.) 


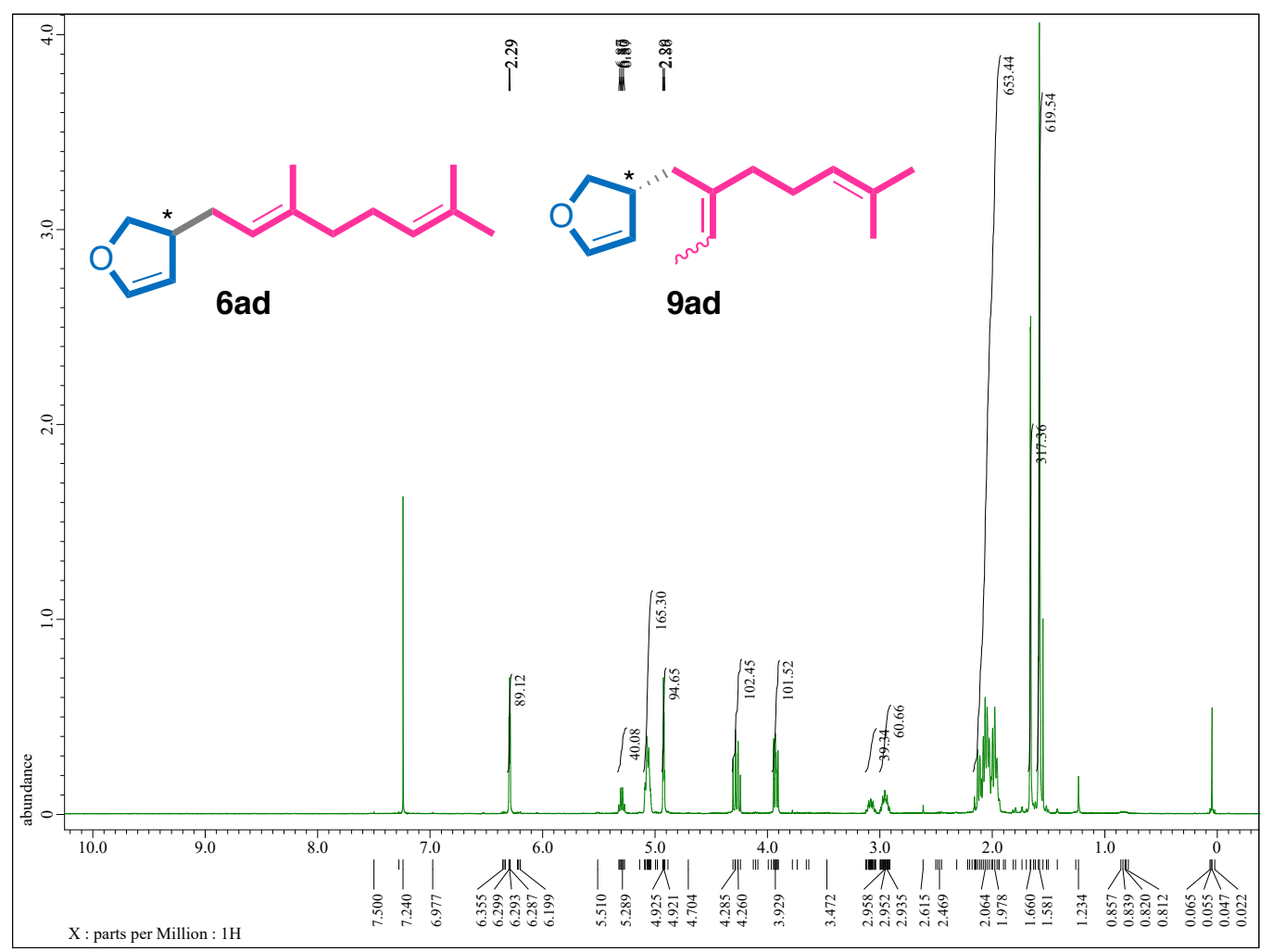

Figure S18-1. 'H NMR Spectrum of 3-(3,7-dimethylocta-2,6-diene-1-yl)-2,3-dihydrofuran (6ad) and 3-(2ethylidene-6-methylhept-5-en-1-yl)-2,3-dihydrofuran (9ad) (400 MHz, $\mathrm{CDCl}_{3}$, r.t.). 


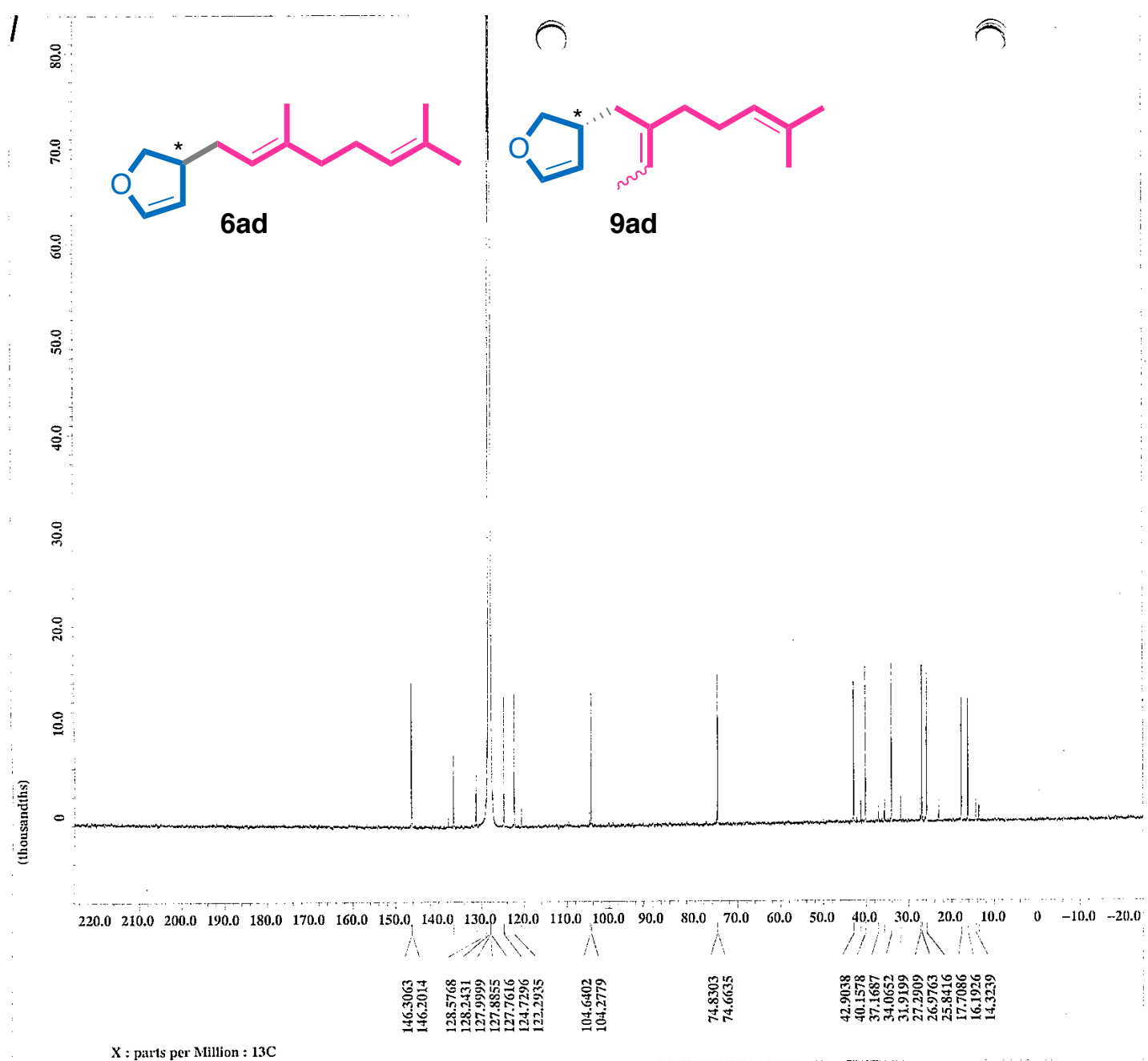

Figure S18-2. ${ }^{13} \mathrm{C}\{1 \mathrm{H}\}$ NMR Spectrum of 3-(3,7-dimethylocta-2,6-diene-1-yl)-2,3-dihydrofuran (6ad) and 3-(2-ethylidene-6-methylhept-5-en-1-yl)-2,3-dihydrofuran (9ad) (100 MHz, $\mathrm{C}_{6} \mathrm{D}_{6}$, r.t.). 


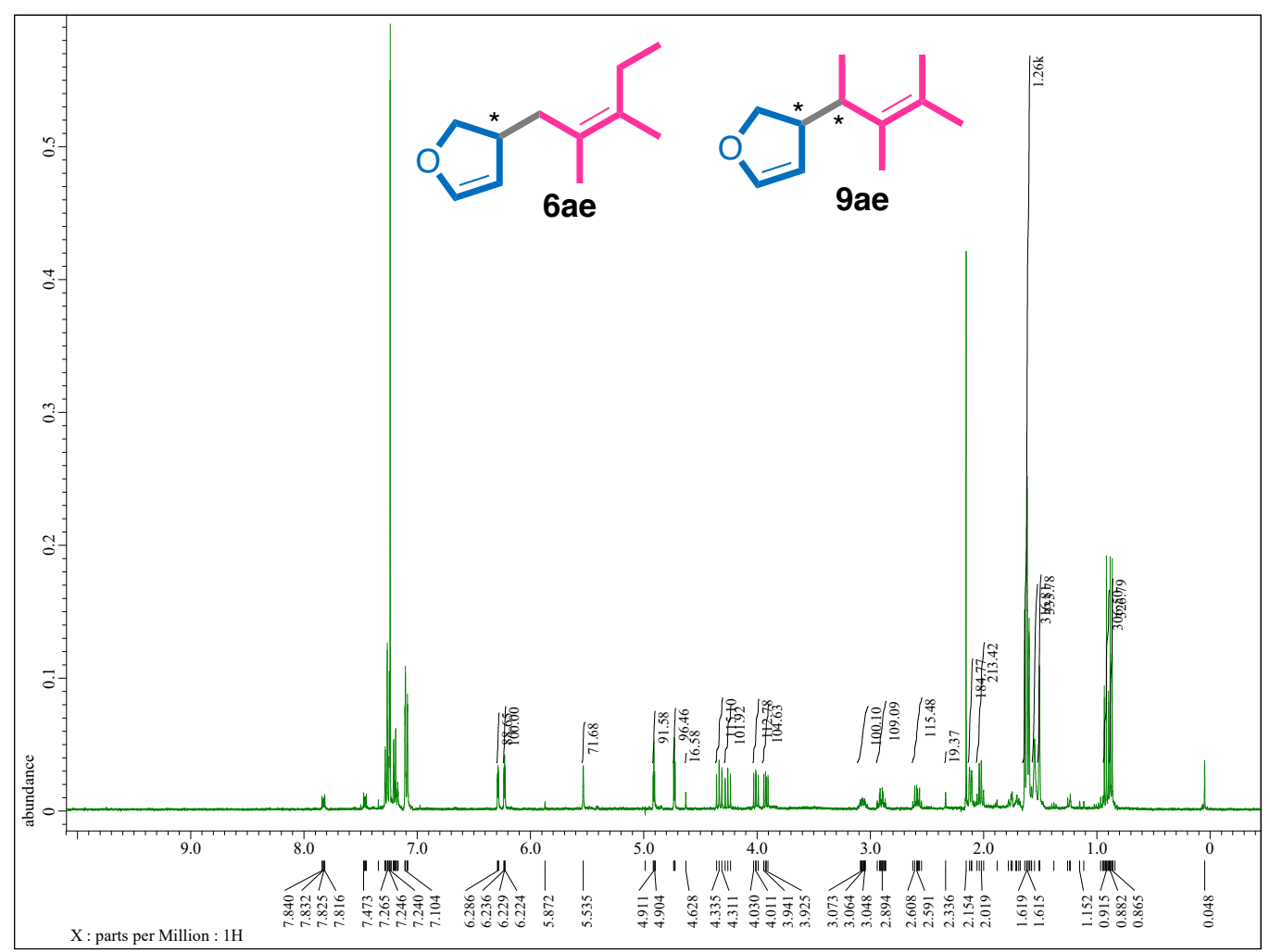

Figure S19-1. ' $\mathrm{H}$ NMR Spectrum of 3-(2,3-dimethylpent-2-en-1-yl)-2,3-dihydrofuran (6ae) and 3-(3,4dimethylpent-3-en-2-yl)-2,3-dihydrofuran (9ae) $\left(400 \mathrm{MHz}, \mathrm{CDCl}_{3}\right.$, r.t.).

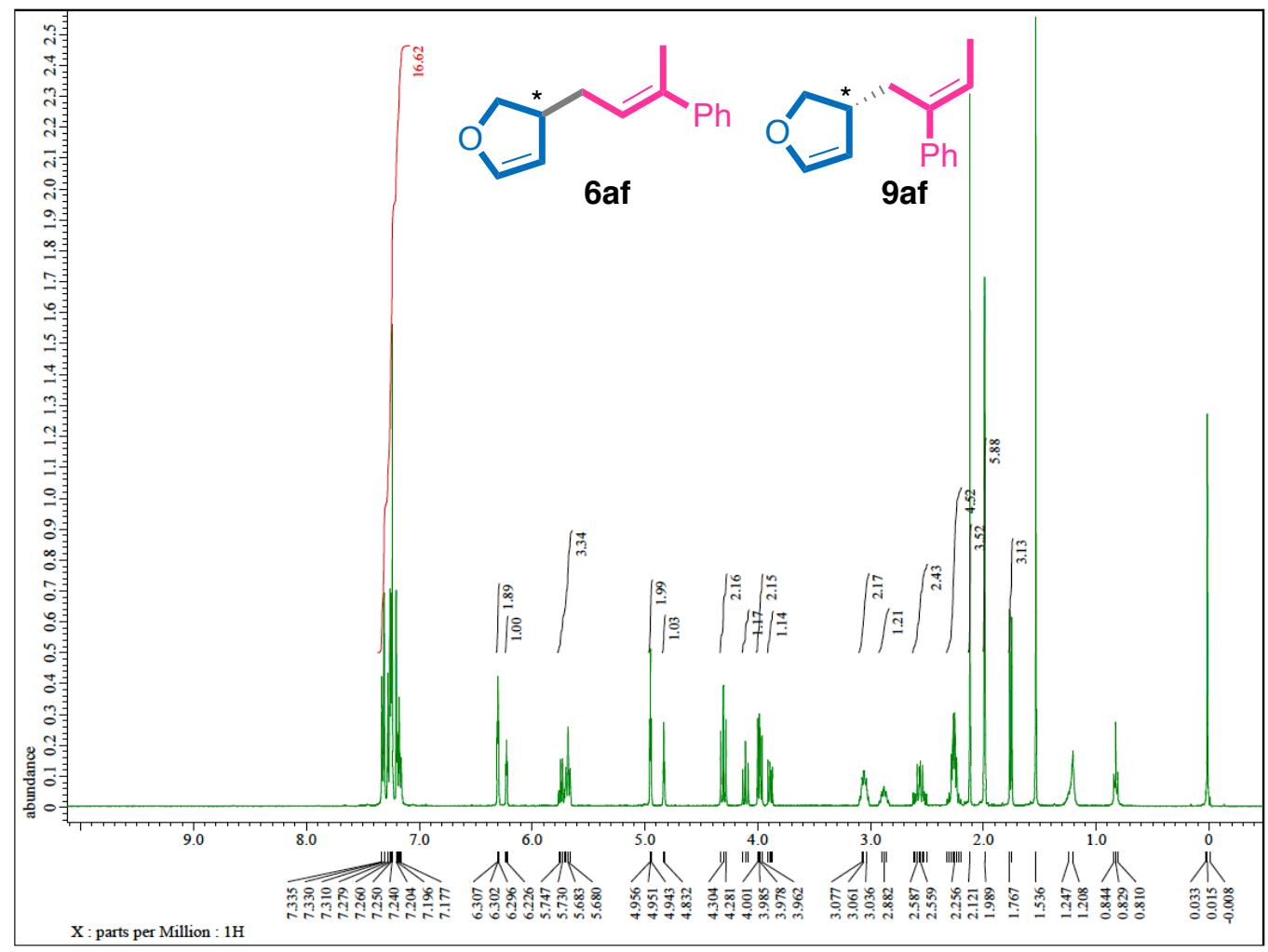

Figure S20-1. ${ }^{1 H}$ NMR Spectrum of (E)-3-(3-phenylbut-2-en-1-yl)-2,3-dihydrofuran (6af) and (E)-3-(2phenylbut-2-en-1-yl)-2,3-dihydrofuran (9af) $\left(400 \mathrm{MHz}, \mathrm{CDCl}_{3}\right.$, r.t.). 


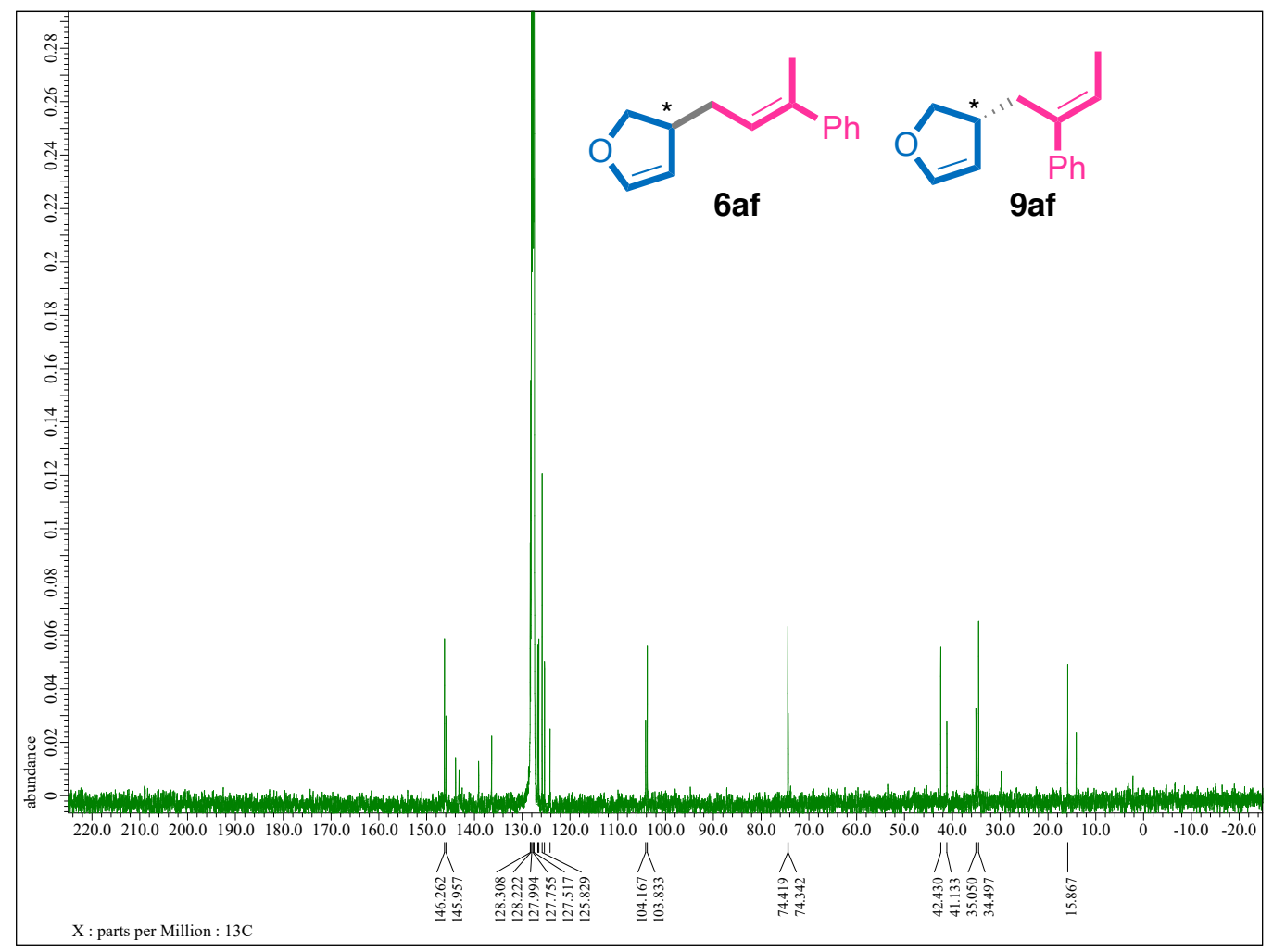

Figure S20-2. ${ }^{13} \mathrm{C}\left\{{ }^{1} \mathrm{H}\right\}$ NMR Spectrum of (E)-3-(3-phenylbut-2-en-1-yl)-2,3-dihydrofuran (6af) and (E)-3(2-phenylbut-2-en-1-yl)-2,3-dihydrofuran (9af) (100 MHz, $\mathrm{C}_{6} \mathrm{D}_{6}$, r.t.). 
Mass Spectrum SmartFormula Report
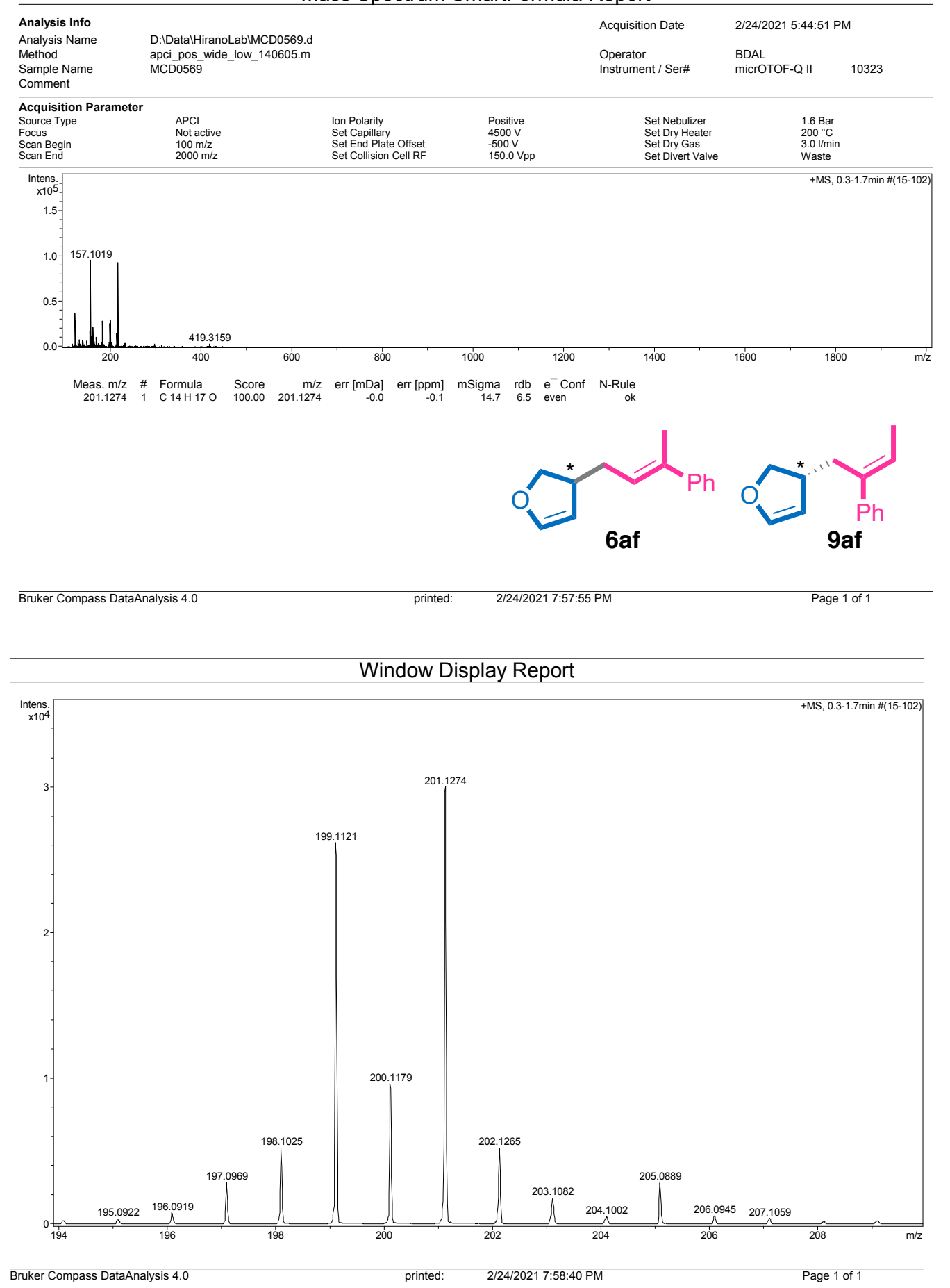

Figure S20-3. HRMS (APCI) Spectrum of (E)-3-(3-phenylbut-2-en-1-yl)-2,3-dihydrofuran (6af) and (E)-3(2-phenylbut-2-en-1-yl)-2,3-dihydrofuran (9af). 


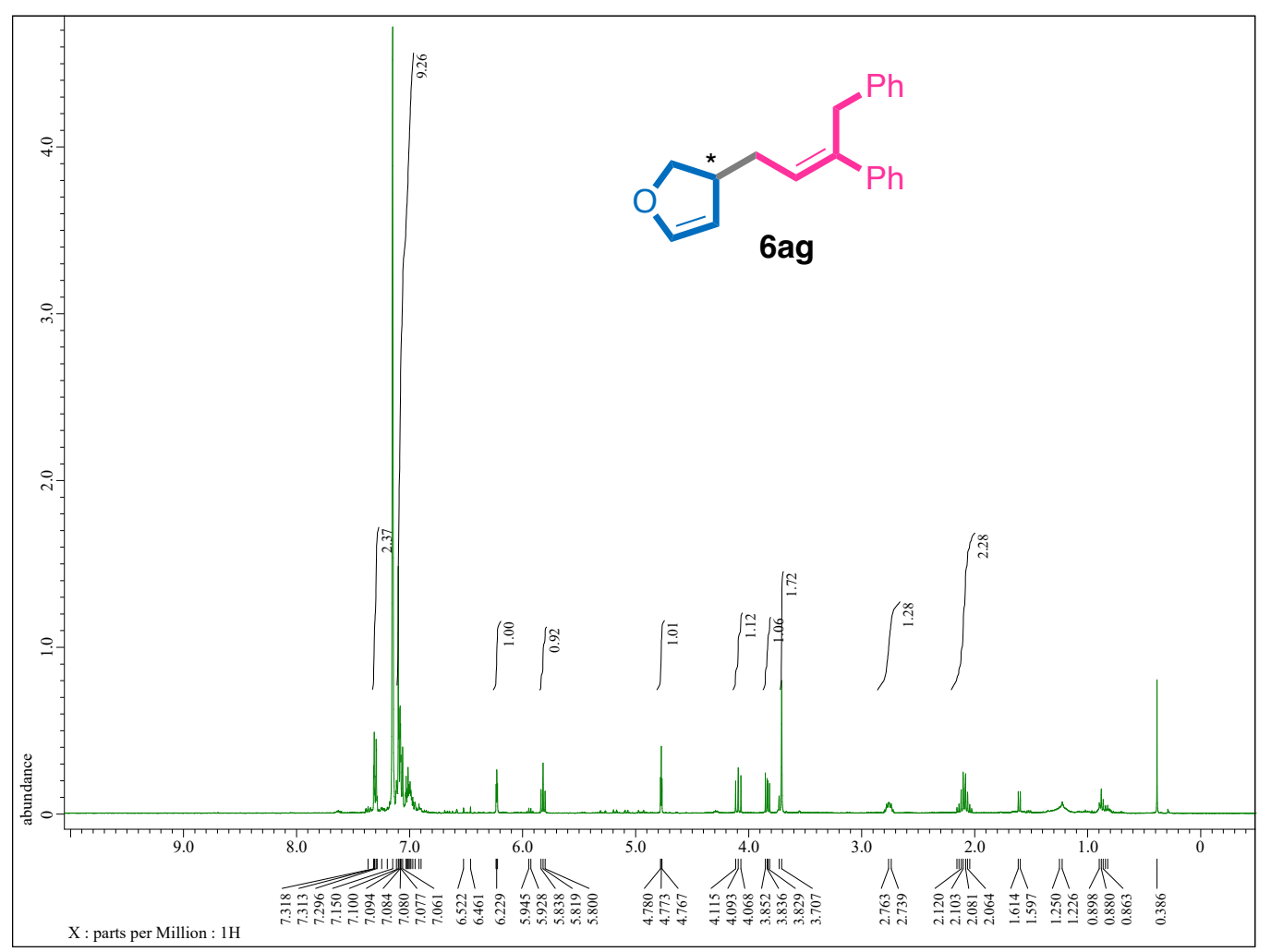

Figure S21-1. 'H NMR Spectrum of (E)-3-(3,4-diphenylbut-2-en-1-yl)-2,3-dihydrofuran (6ag) $(400 \mathrm{MHz}$, $\mathrm{CDCl}_{3}$, r.t.).

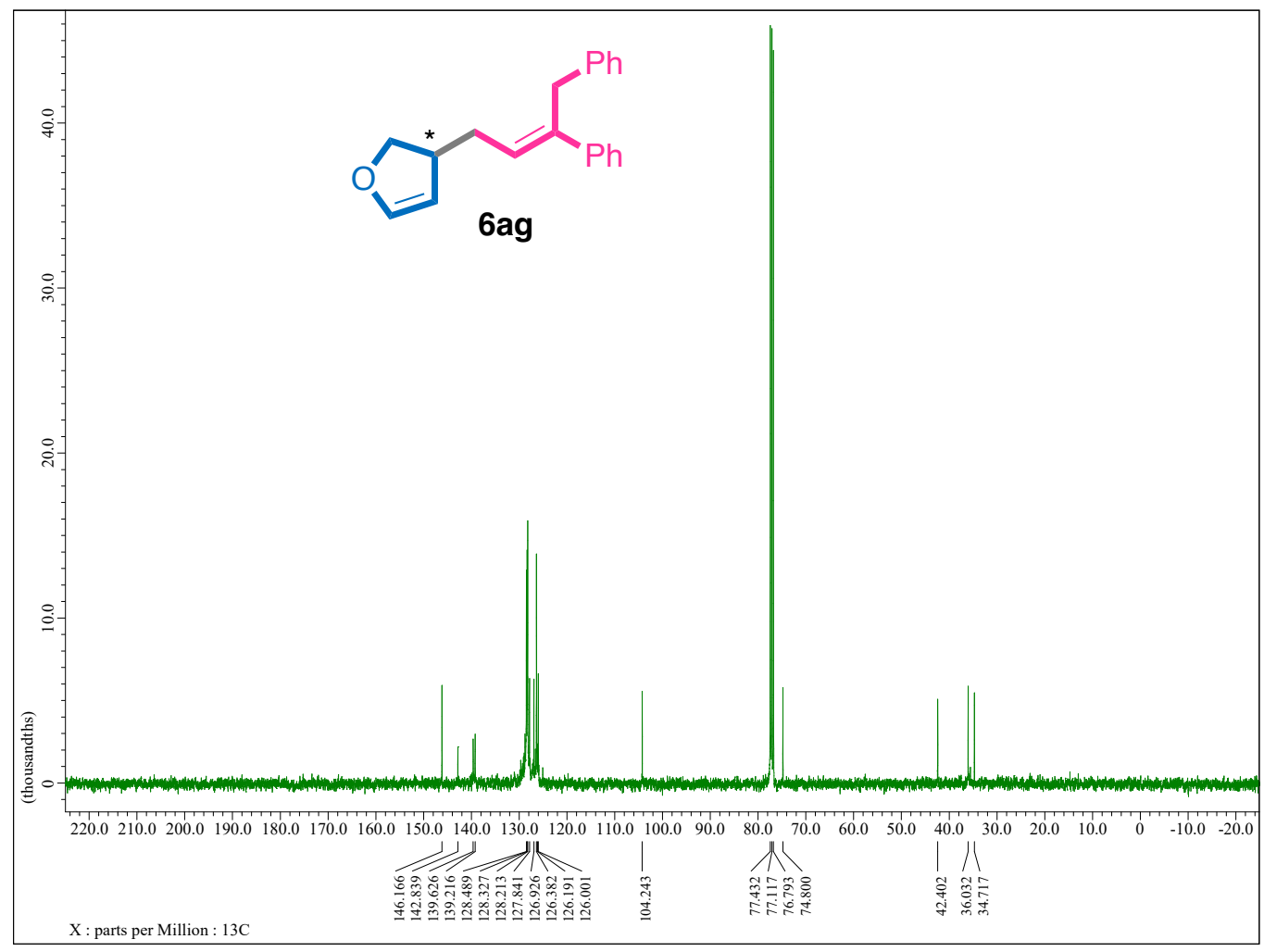

Figure S21-2. ${ }^{13} \mathrm{C}\left\{{ }^{1} \mathrm{H}\right\}$ NMR Spectrum of (E)-3-(3,4-diphenylbut-2-en-1-yl)-2,3-dihydrofuran (6ag) (100 $\mathrm{MHz}, \mathrm{CDCl}_{3}$, r.t.). 


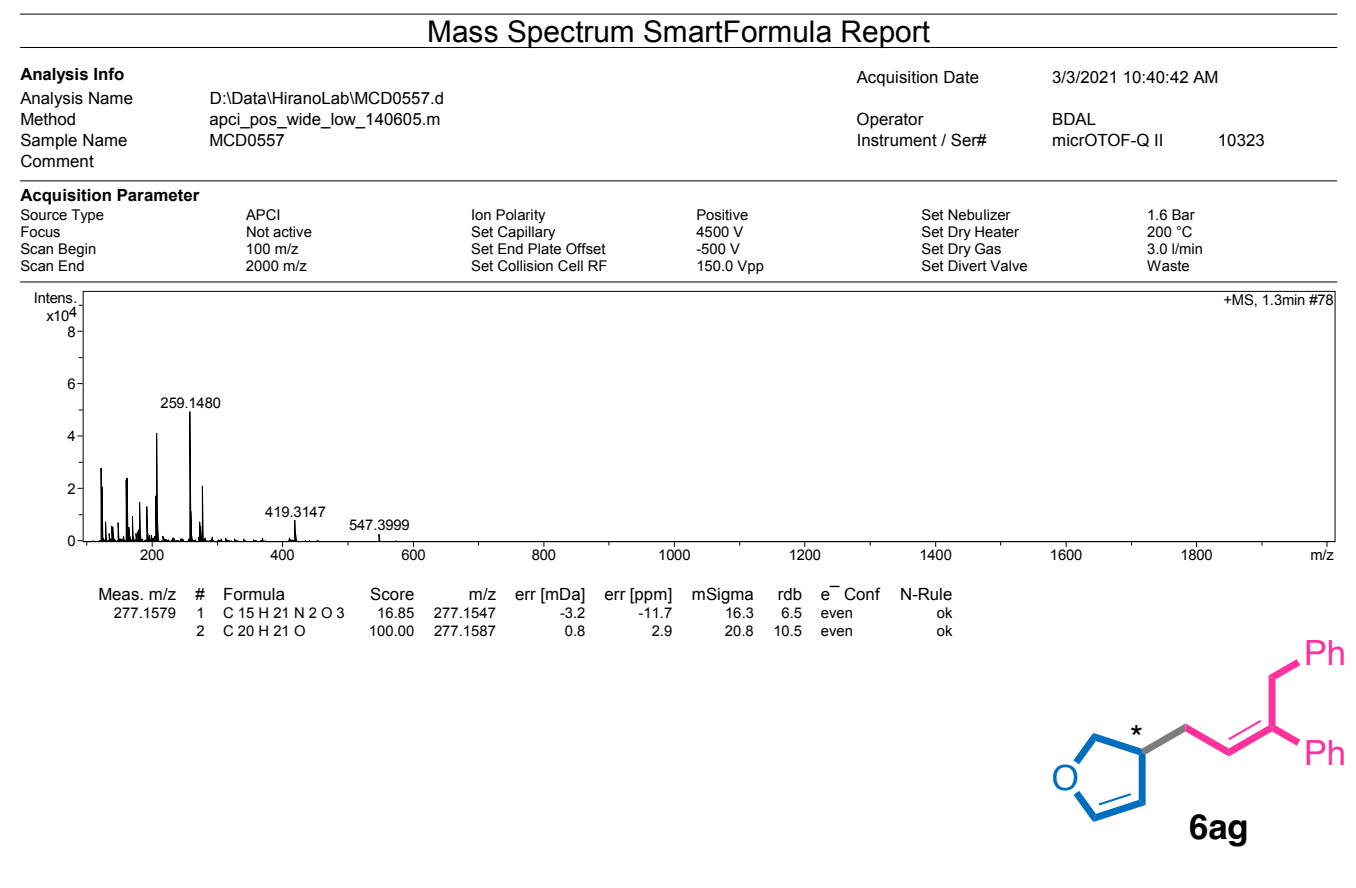

\begin{tabular}{lll}
\hline Bruker Compass DataAnalysis 4.0 4.0 & printed: & $3 / 3 / 2021$ 10:46:46 AM 1 of 1
\end{tabular}

Window Display Report

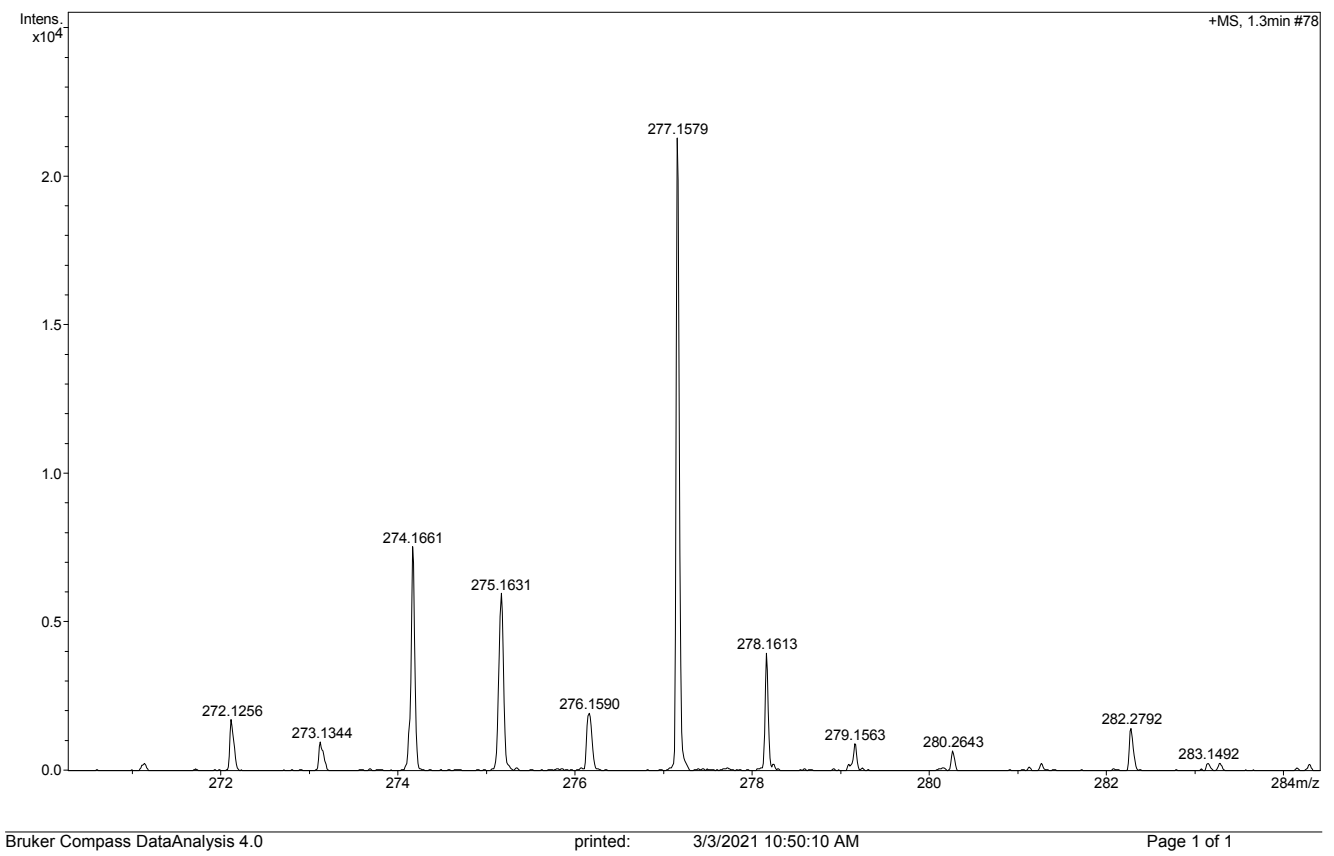

Figure S21-3. HRMS (APCI) Spectrum of (E)-3-(3,4-diphenylbut-2-en-1-yl)-2,3-dihydrofuran (6ag). 


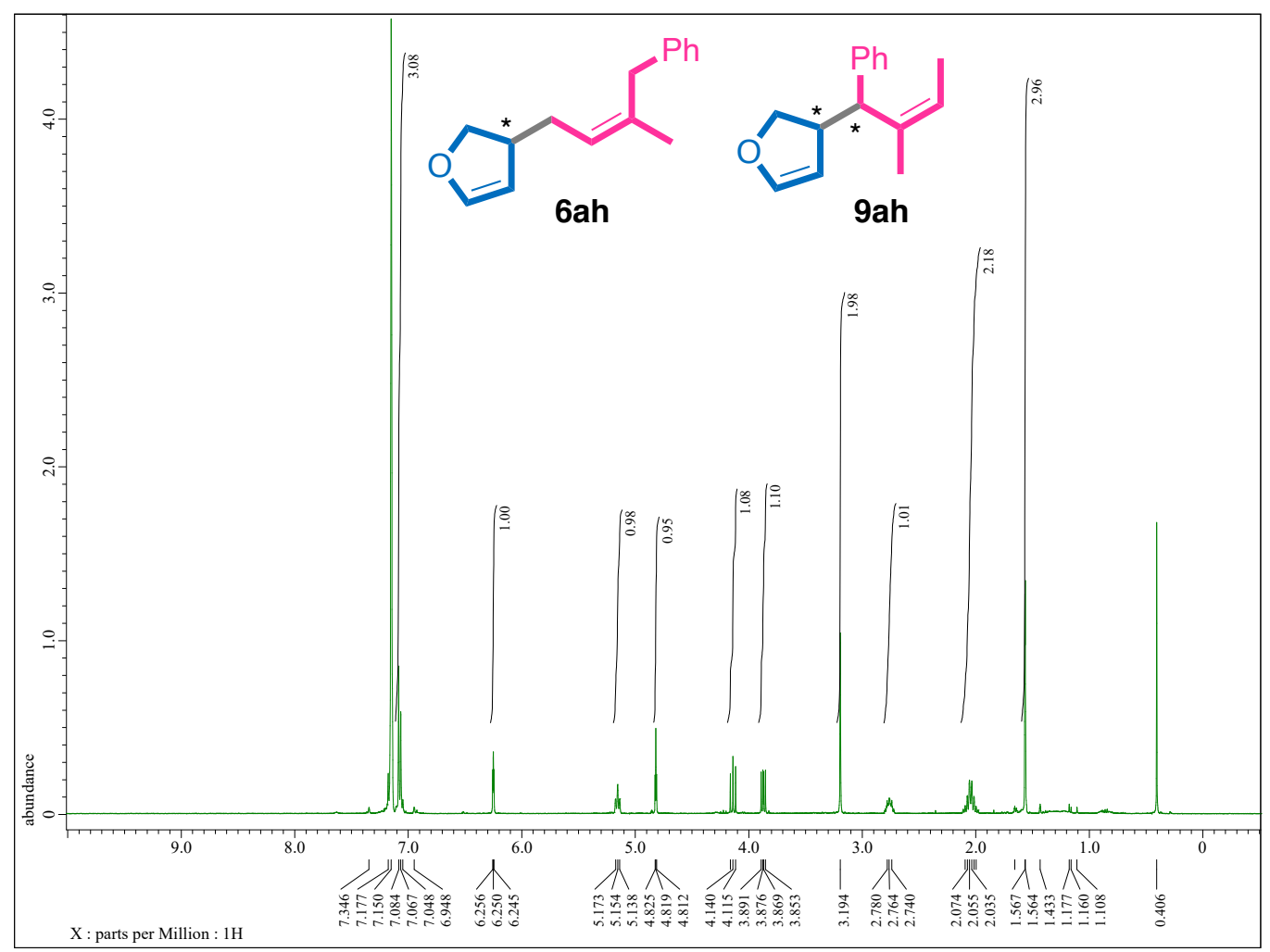

Figure S22-1. 'H NMR Spectrum of (Z)-3-(3-methyl-4-phenylbut-2-en-1-yl)-2,3-dihydrofuran (6ah) and (Z)-3-(2-methyl-1-phenylbut-2-en-1-yl)-2,3-dihydrofuran (9ah) (400 MHz, $\mathrm{C}_{6} \mathrm{D}_{6}$, r.t.). 


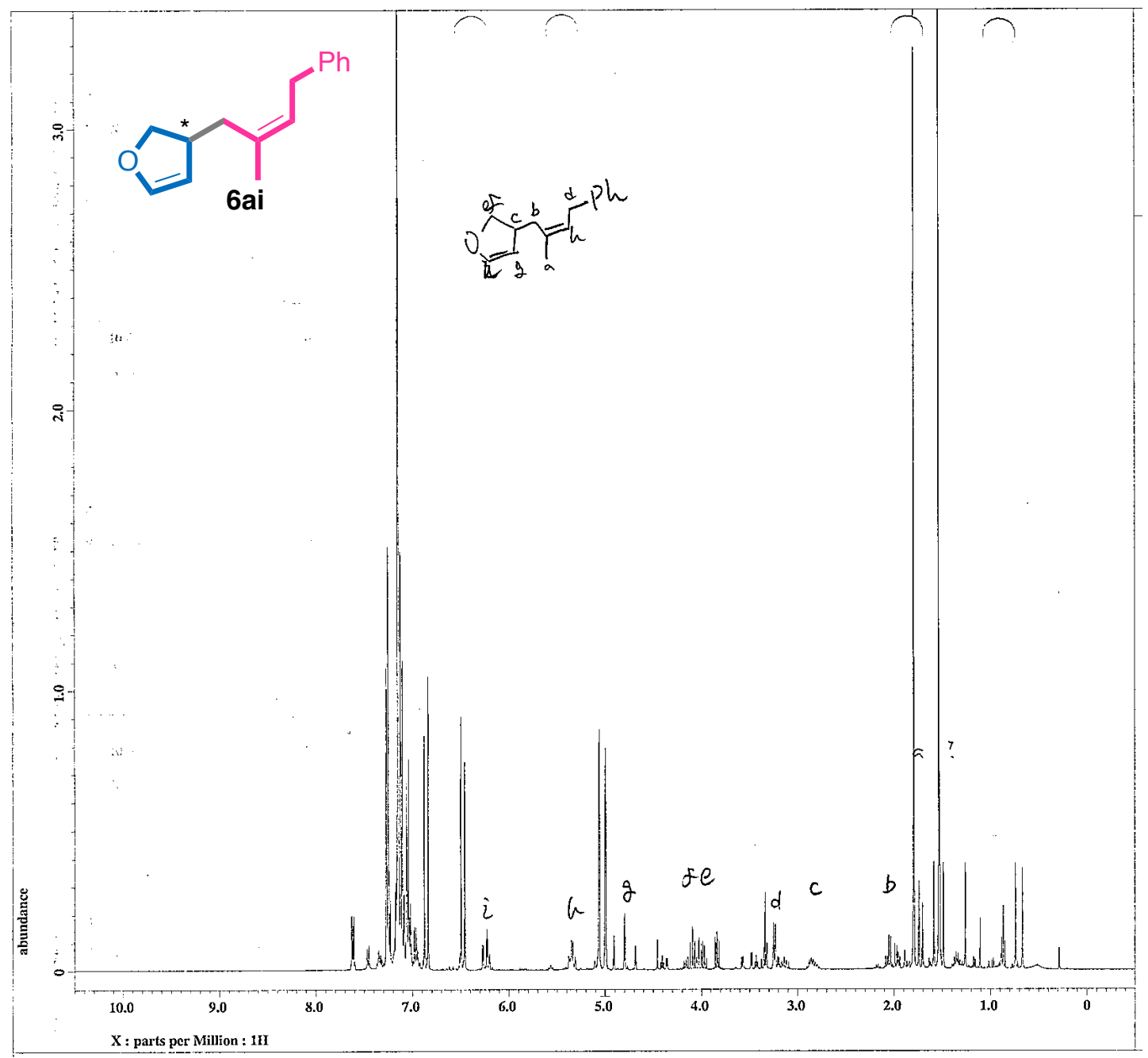

Figure S23-1. 'H NMR Spectrum of (Z)-3-(2-methyl-4-phenylbut-2-en-1-yl)-2,3-dihydrofuran (6ai) (400 $\mathrm{MHz}, \mathrm{C}_{6} \mathrm{D}_{6}$, r.t.). 


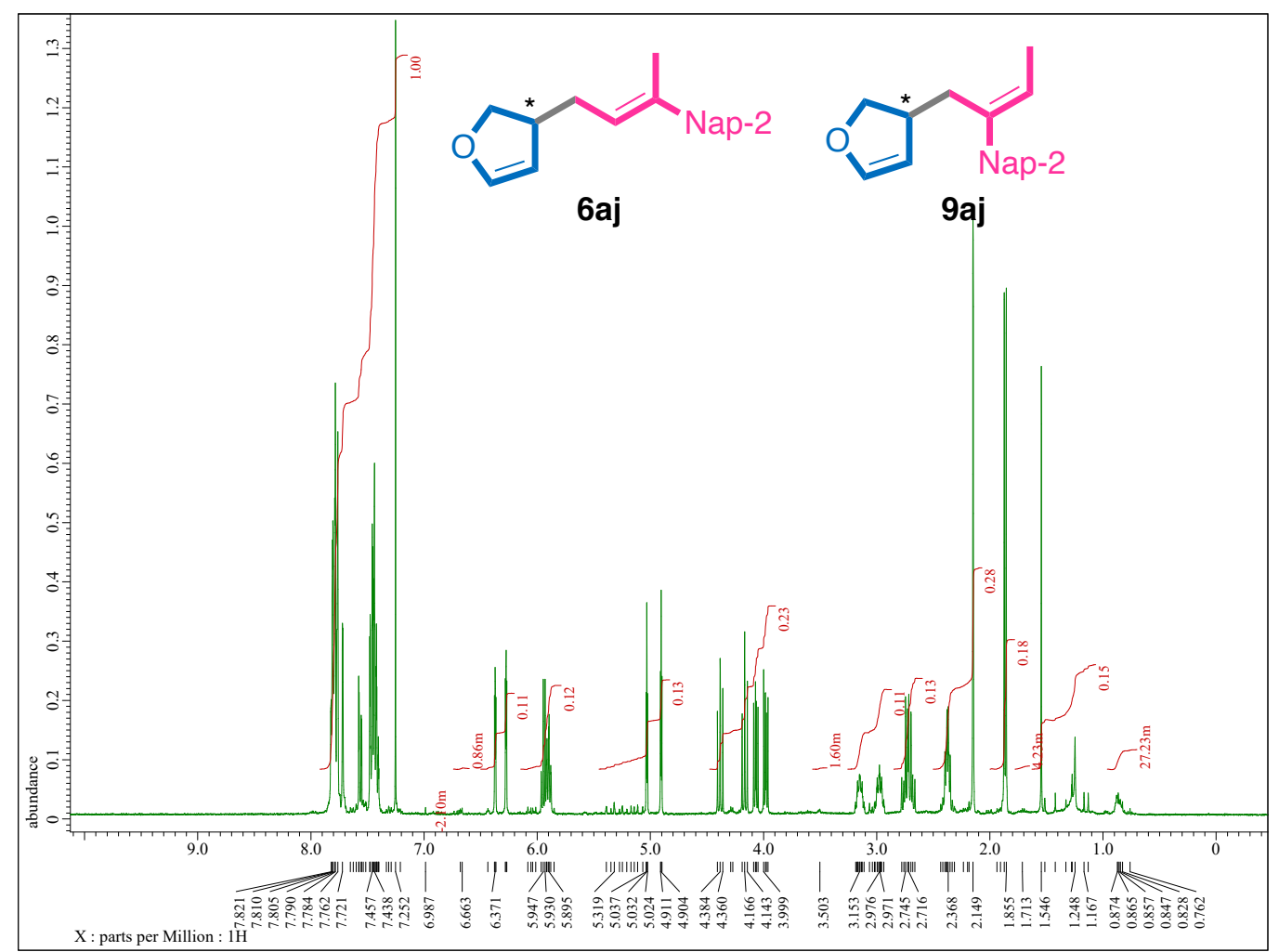

Figure S24-1. ${ }^{1} \mathrm{H}$ NMR Spectrum of (E)-3-(3-2-naphthylbut-2-en-1-yl)-2,3-dihydrofuran (6aj) and (E)-3-(22-naphthylbut-2-en-1-yl)-2,3-dihydrofuran (9aj) (400 MHz, $\mathrm{CDCl}_{3}$, r.t.).

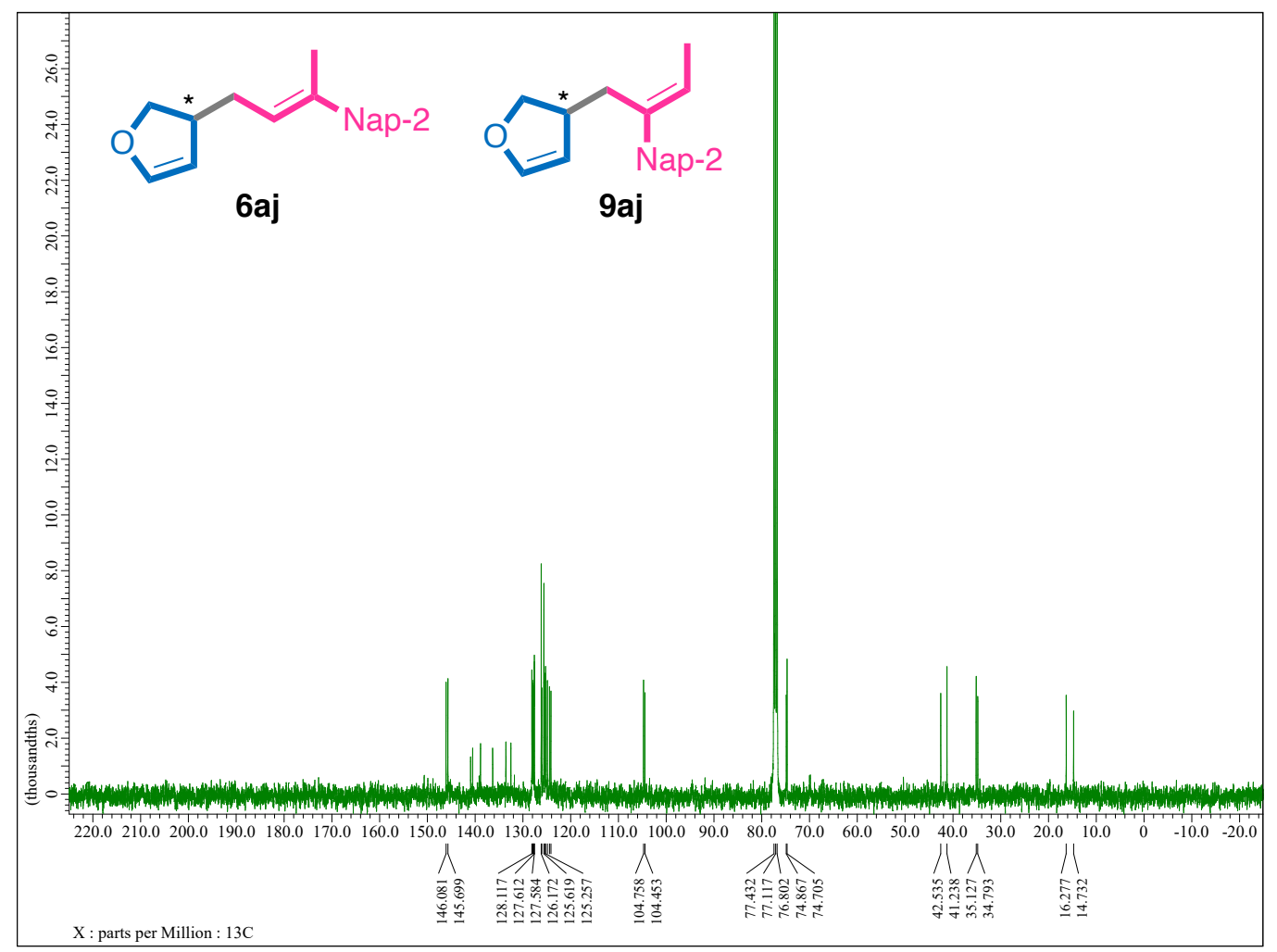

Figure S24-2. ${ }^{13} C\left\{{ }^{1} H\right\}$ NMR Spectrum of $(E)$-3-(3-2-naphthylbut-2-en-1-yl)-2,3-dihydrofuran (6aj) and $(E)$ 3-(2-2-naphthylbut-2-en-1-yl)-2,3-dihydrofuran (9aj) (100 MHz, $\mathrm{CDCl}_{3}$, r.t.) 


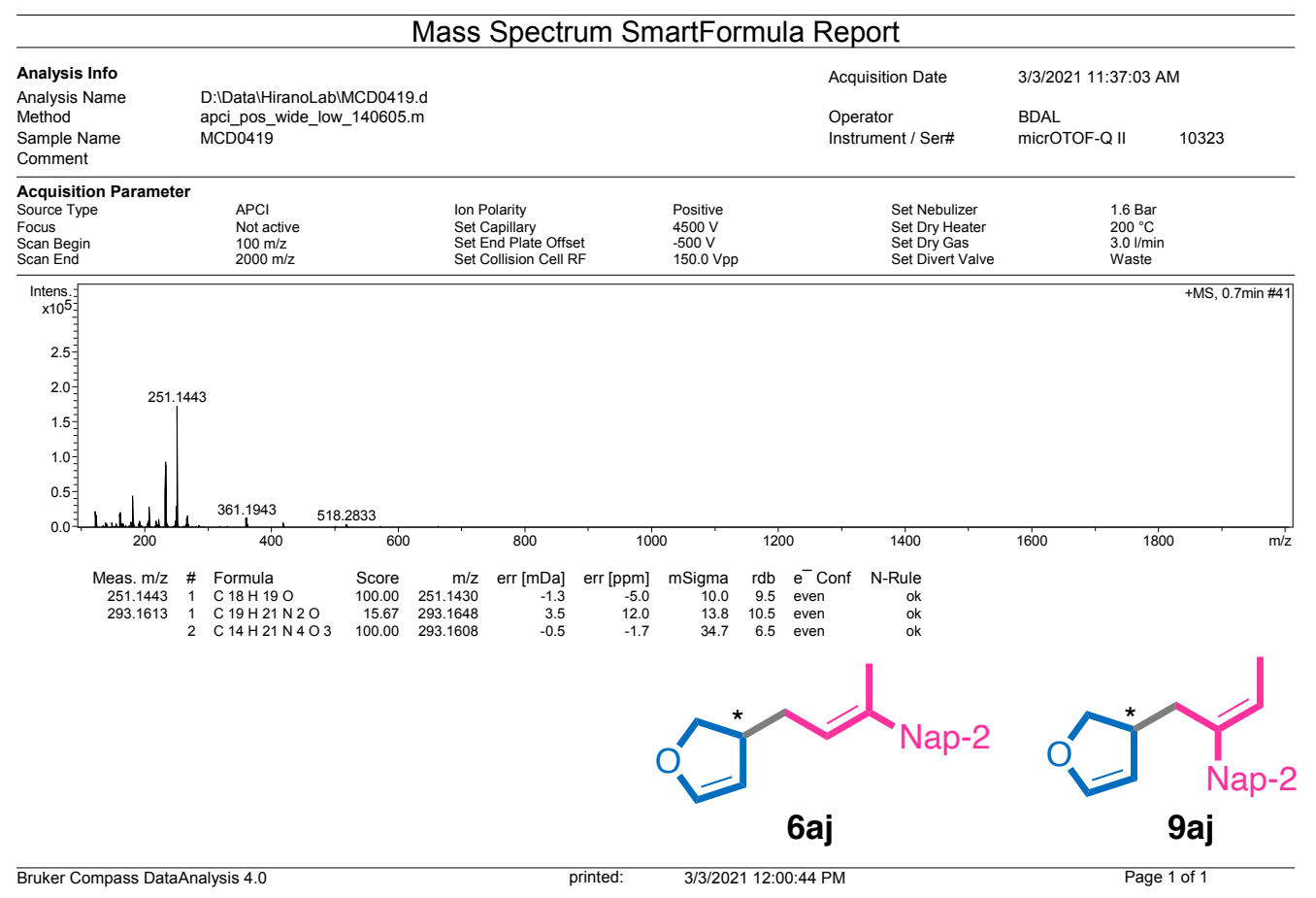

Figure S24-3. HRMS (APCI) Spectrum of (E)-3-(3-2-naphthylbut-2-en-1-yl)-2,3-dihydrofuran (6aj) and (E)-3-(2-2-naphthylbut-2-en-1-yl)-2,3-dihydrofuran (9aj)

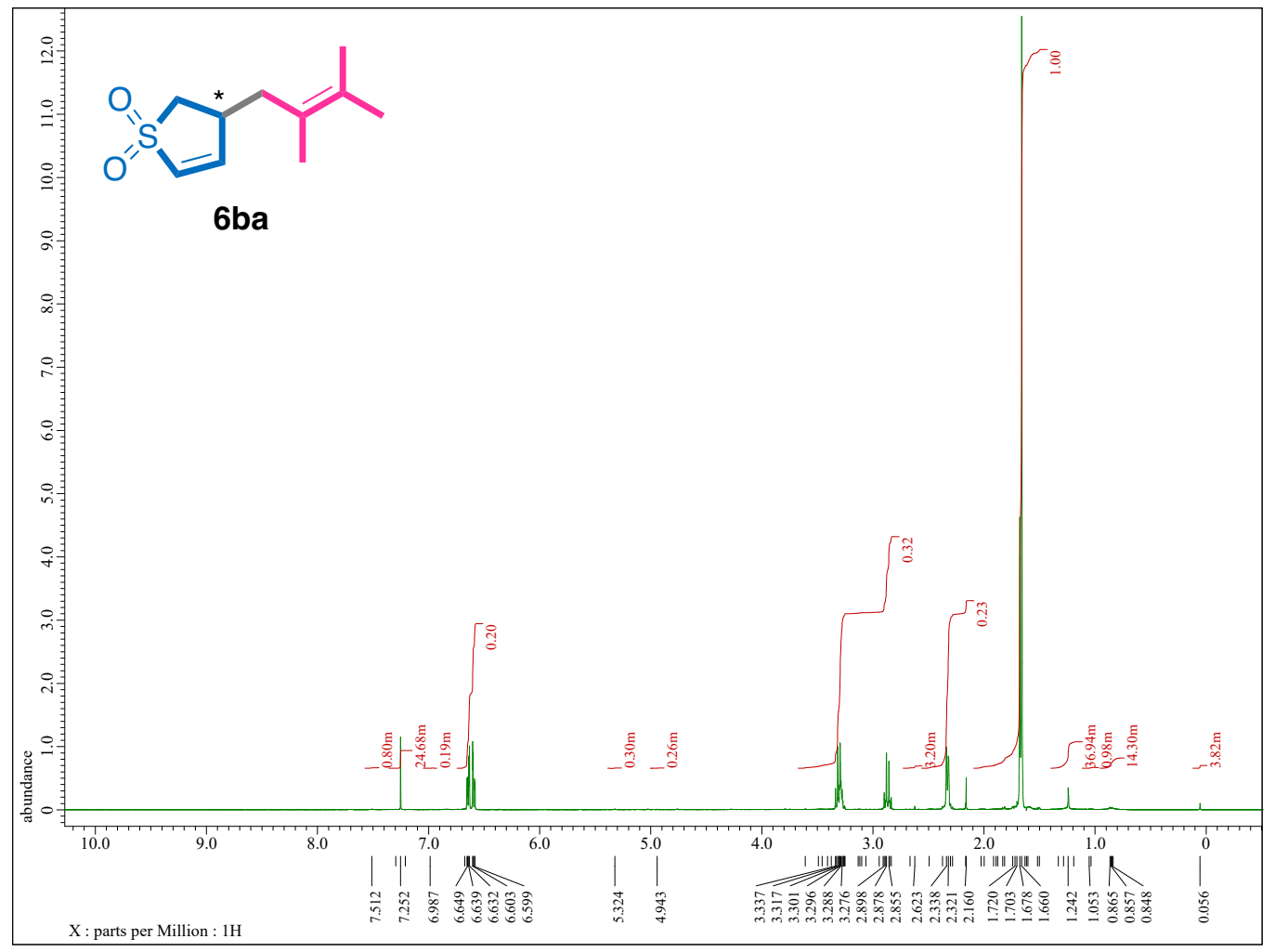

Figure S25-1. 'H NMR Spectrum of 3-(2,3-dimethylbut-2,3-en-1-yl)-2,3-dihydrothiophene 1,1-dioxide (6ba) $\left(400 \mathrm{MHz}, \mathrm{CDCl}_{3}\right.$, r.t.). 


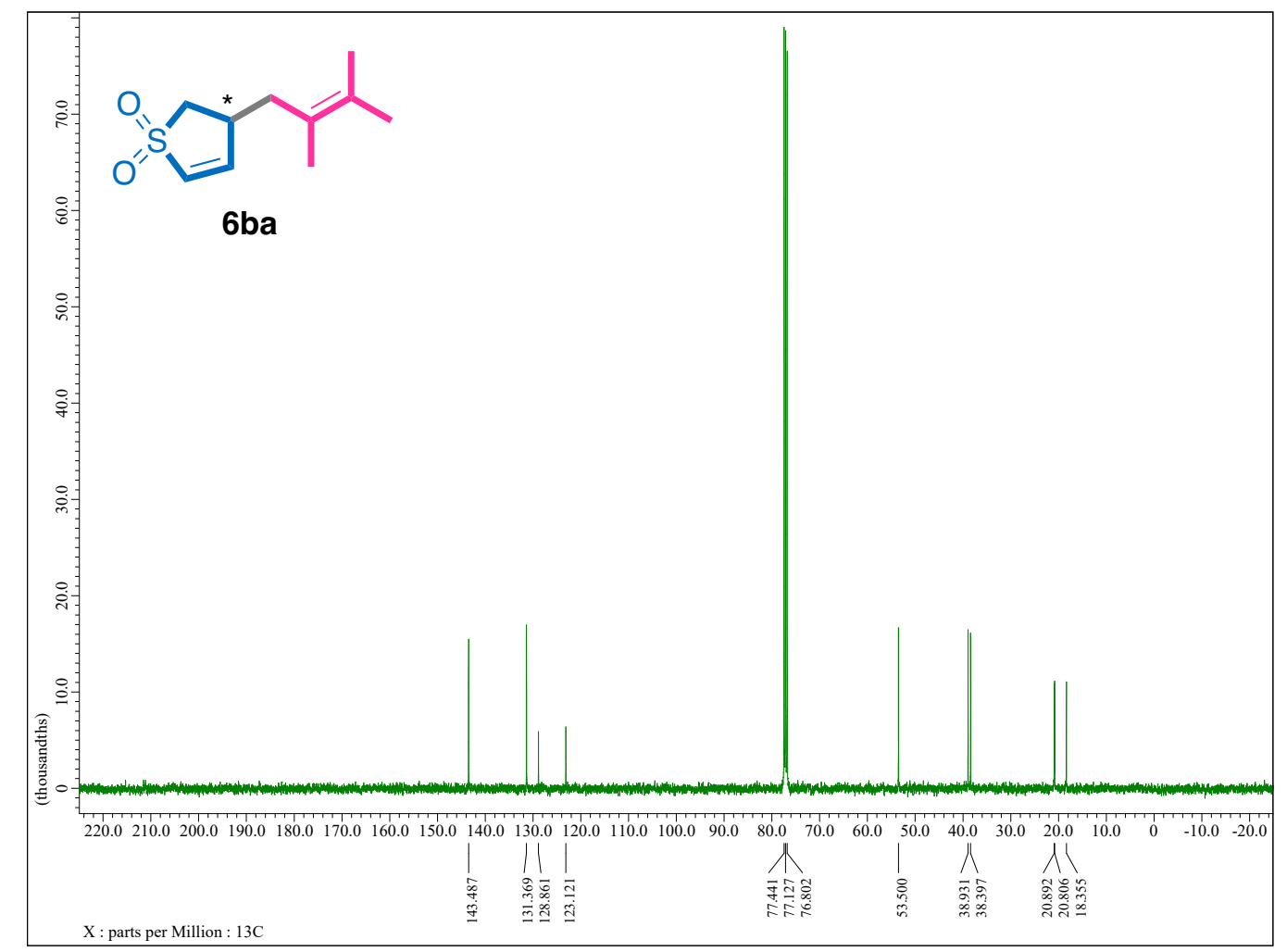

Figure S25-2. ${ }^{13} \mathrm{C}\{\mathrm{H} H$ NMR Spectrum of 3-(2,3-dimethylbut-2,3-en-1-yl)-2,3-dihydrothiophene 1,1dioxide (6ba) $\left(100 \mathrm{MHz}, \mathrm{CDCl}_{3}\right.$, r.t.).

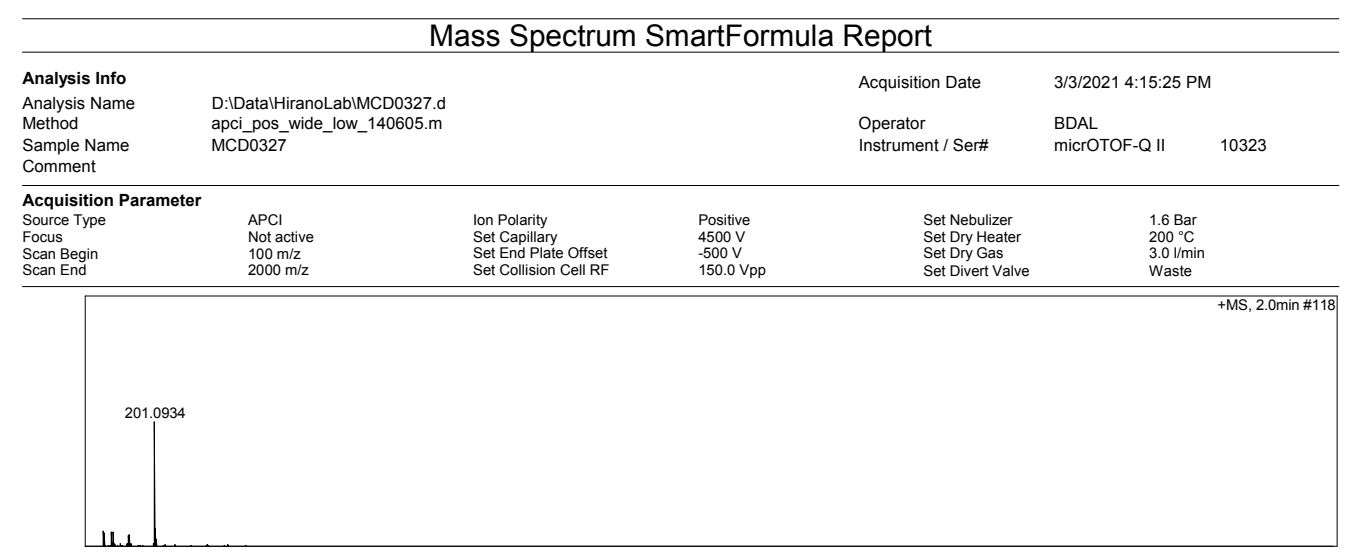

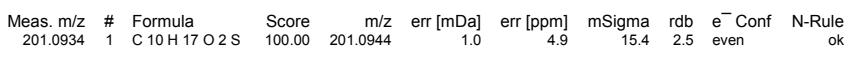

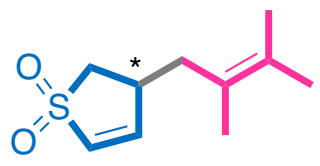

$6 \mathrm{ba}$

Bruker Compass DataAnalysis 4.0

printed: $\quad 3 / 3 / 20214: 23: 41 \mathrm{PM}$

Page 1 of 1

Figure S25-3. HRMS (APCI) Spectrum of 3-(2,3-dimethylbut-2,3-en-1-yl)-2,3-dihydrothiophene 1,1dioxide (6ba). 


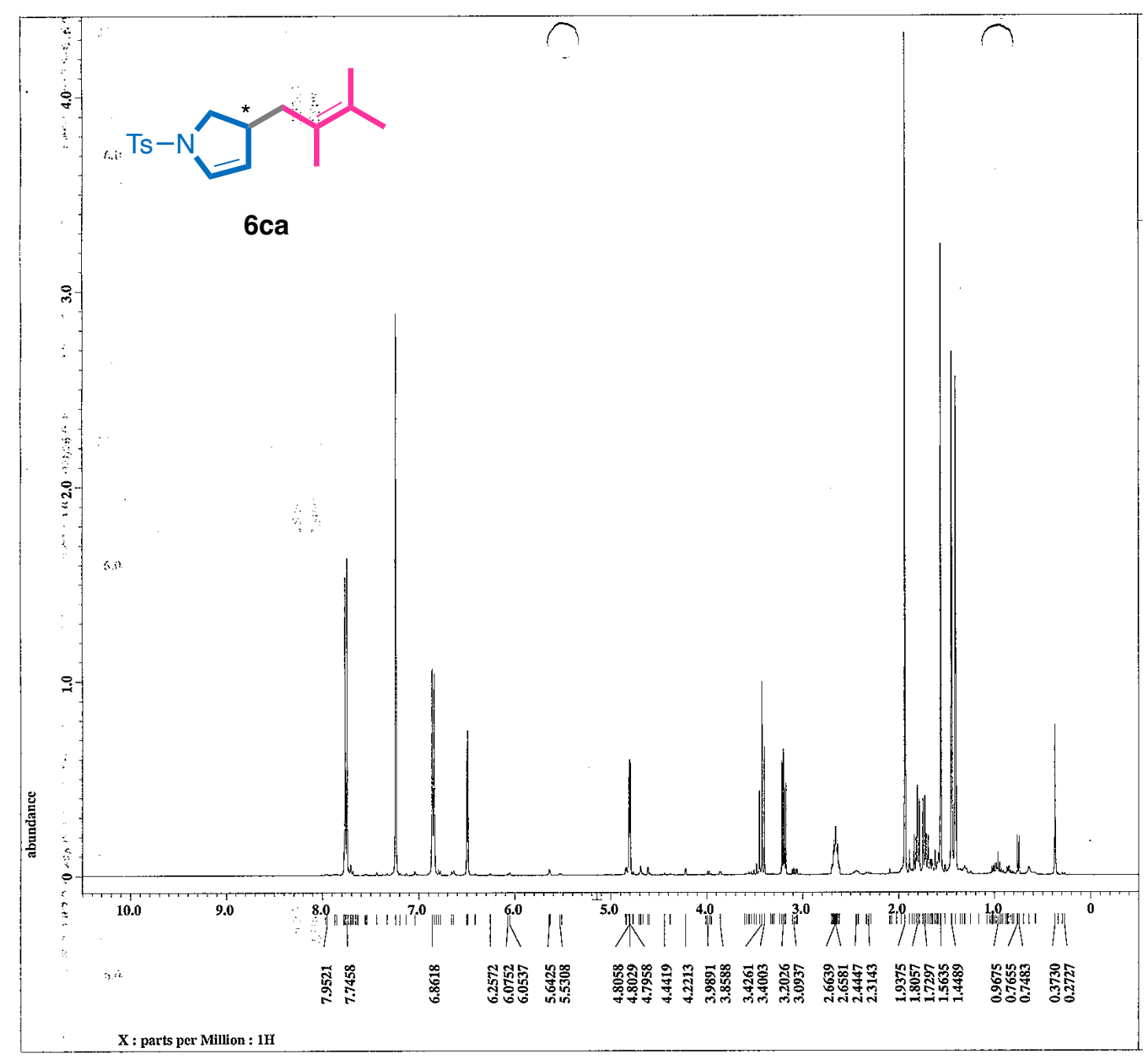

Figure S26-1. ' $\mathrm{H}$ NMR Spectrum of 3-(2,3-dimethylbut-2,3-en-1-yl)-1-tosyl-2,3-dihydro-1H-pyrrole (6ca) (400 MHz, $\mathrm{CDCl}_{3}$, r.t.). 


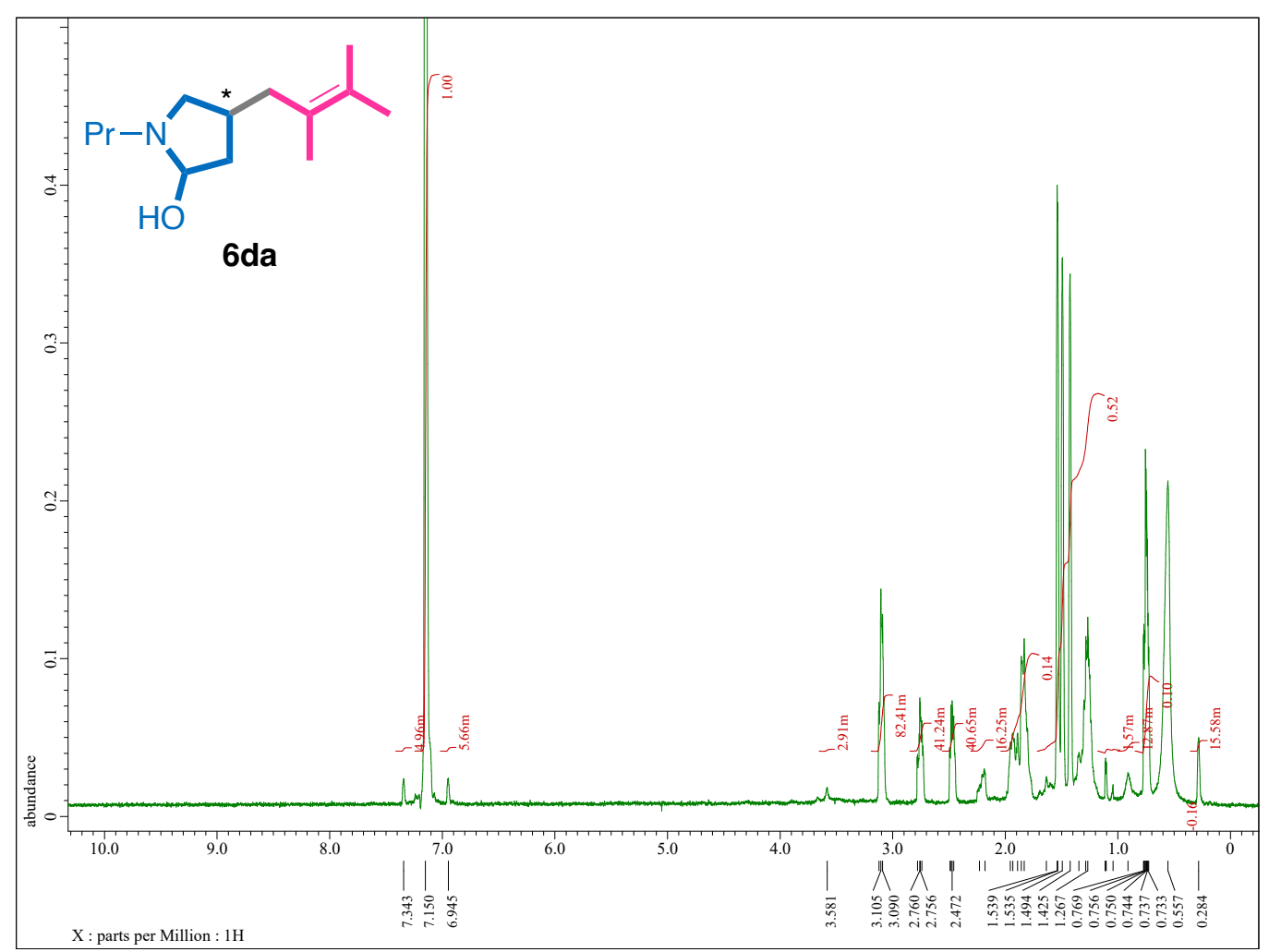

Figure S27-1. ${ }^{1} \mathrm{H}$ NMR of 4-(2,3-dimethylbut-2-en-1-yl)-1-propylpyrrolidin-2-ol (6da) $\left(400 \mathrm{MHz}, \mathrm{C}_{6} \mathrm{D}_{6}\right.$, r.t.).

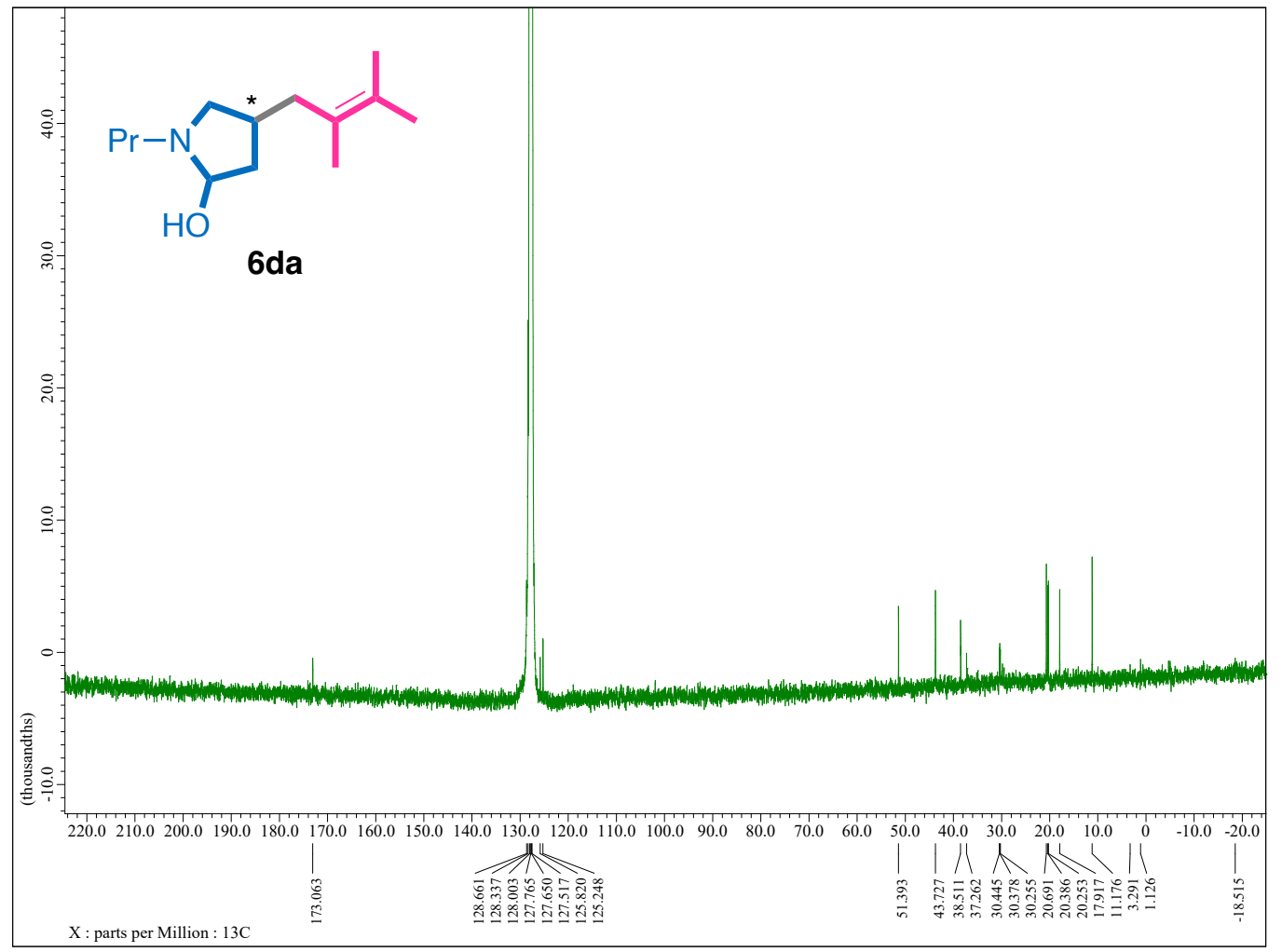

Figure S27-2. ${ }^{13} \mathrm{C}\left\{{ }^{1} \mathrm{H}\right\}$ NMR of 4-(2,3-dimethylbut-2-en-1-yl)-1-propylpyrrolidin-2-ol (6da) $(100 \mathrm{MHz}$, $\mathrm{C}_{6} \mathrm{D}_{6}$, r.t.). 


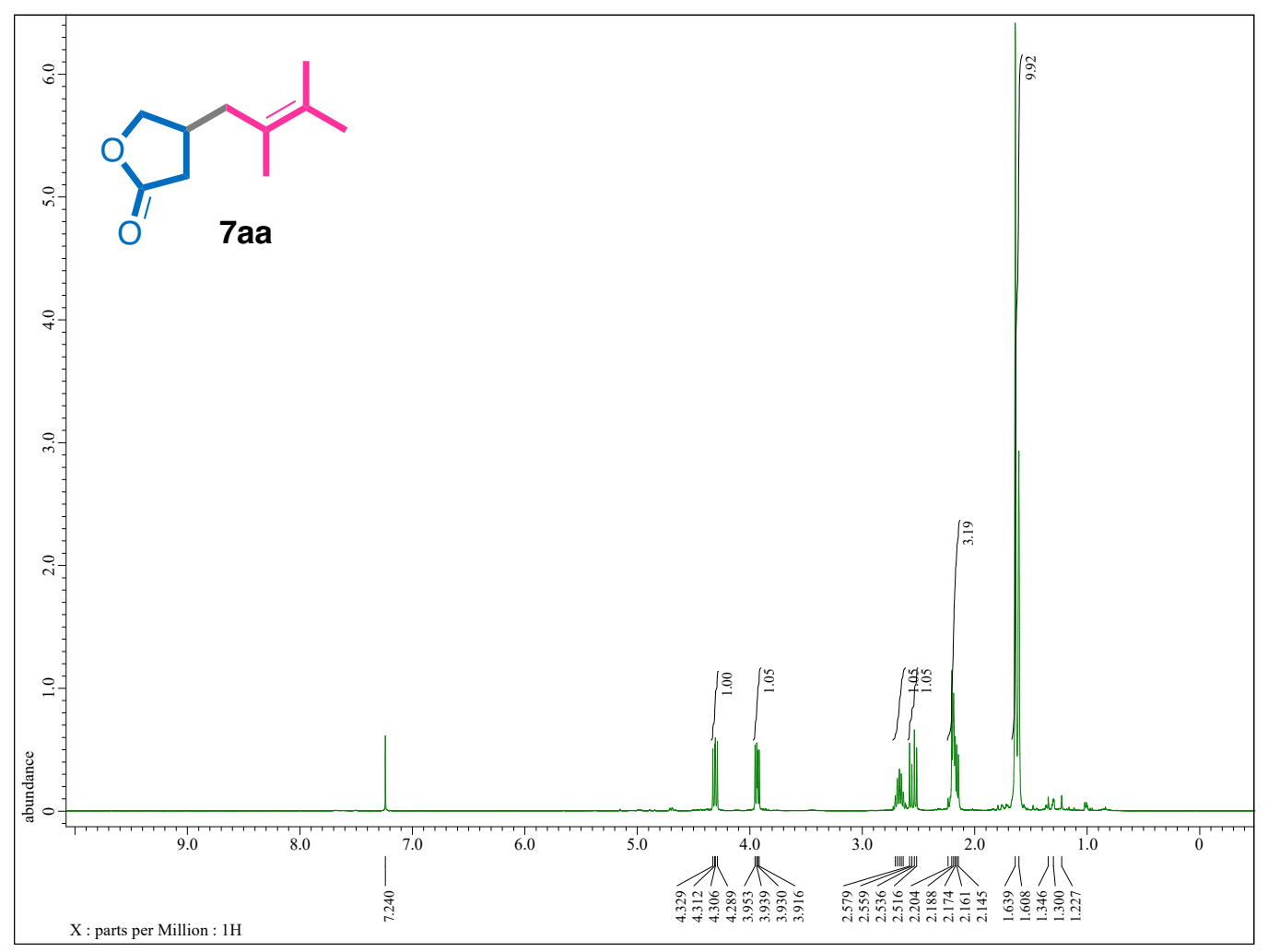

Figure S28-1. ${ }^{1} \mathrm{H}$ NMR Spectrum of rac-4-(2,3-dimethylbu-2-en-1-yl)dihydrofuran-2(3H)-one (rac-7aa) (400 MHz, $\mathrm{CDCl}_{3}$, r.t.).

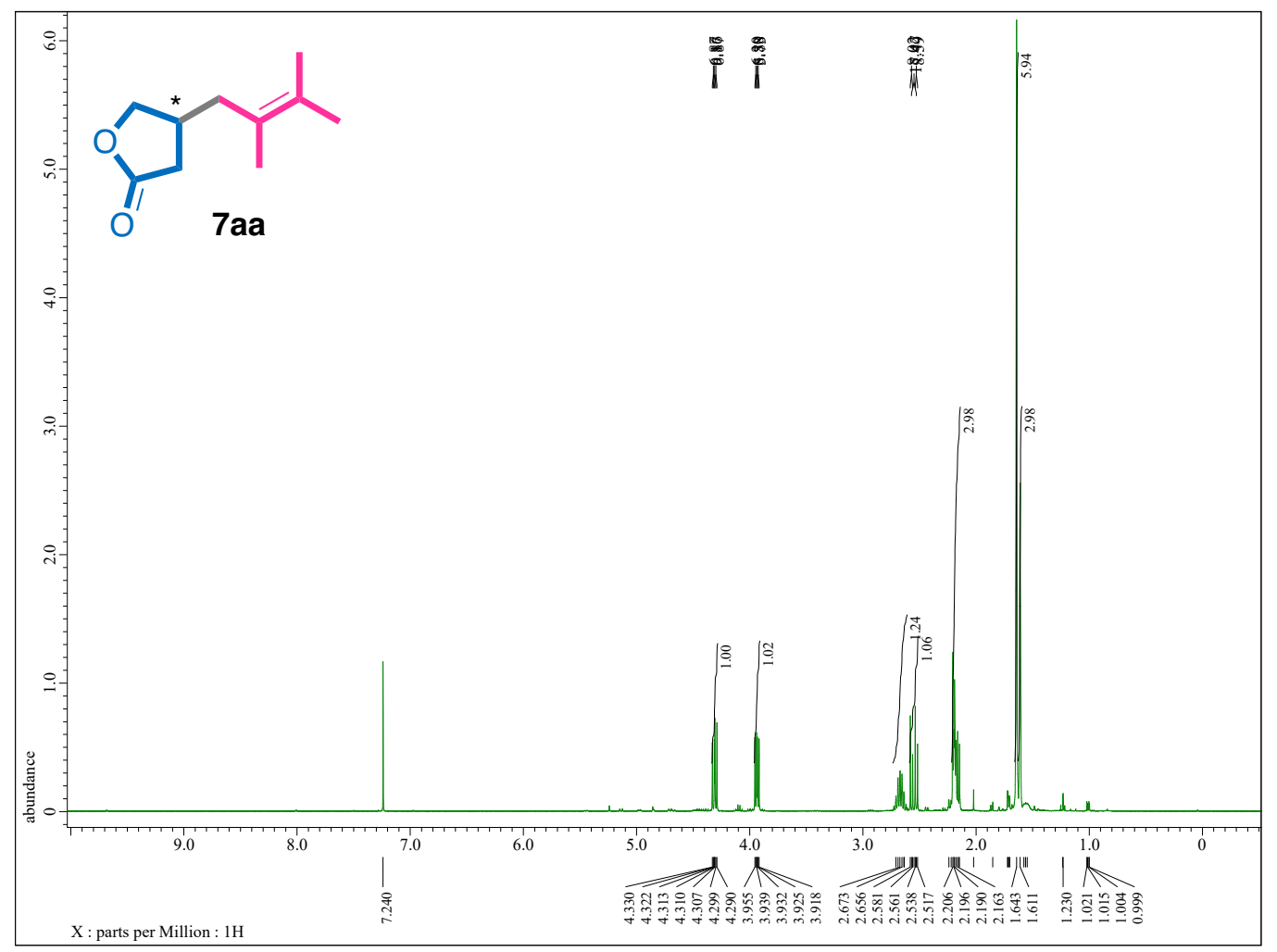

Figure S28-2. ${ }^{1} \mathrm{H}$ NMR Spectrum of ent-4-(2,3-dimethylbu-2-en-1-yl)dihydrofuran-2(3H)-one (ent-7aa) ( $400 \mathrm{MHz}, \mathrm{CDCl}_{3}$, r.t.). 


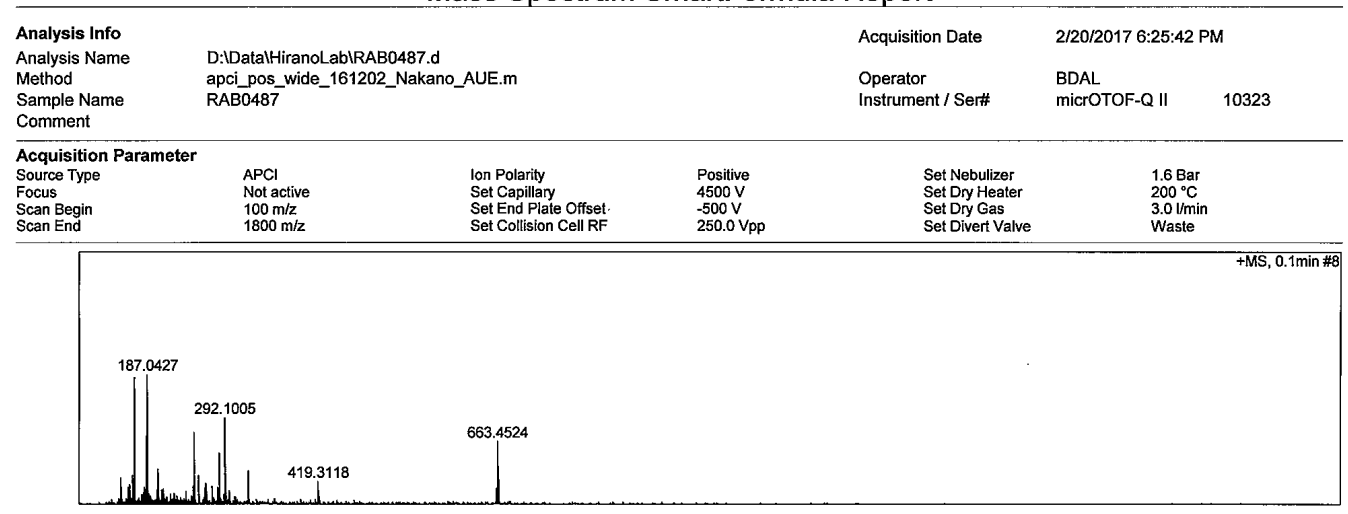

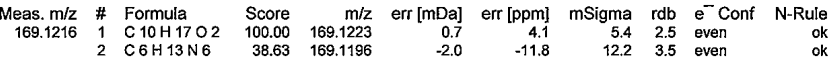
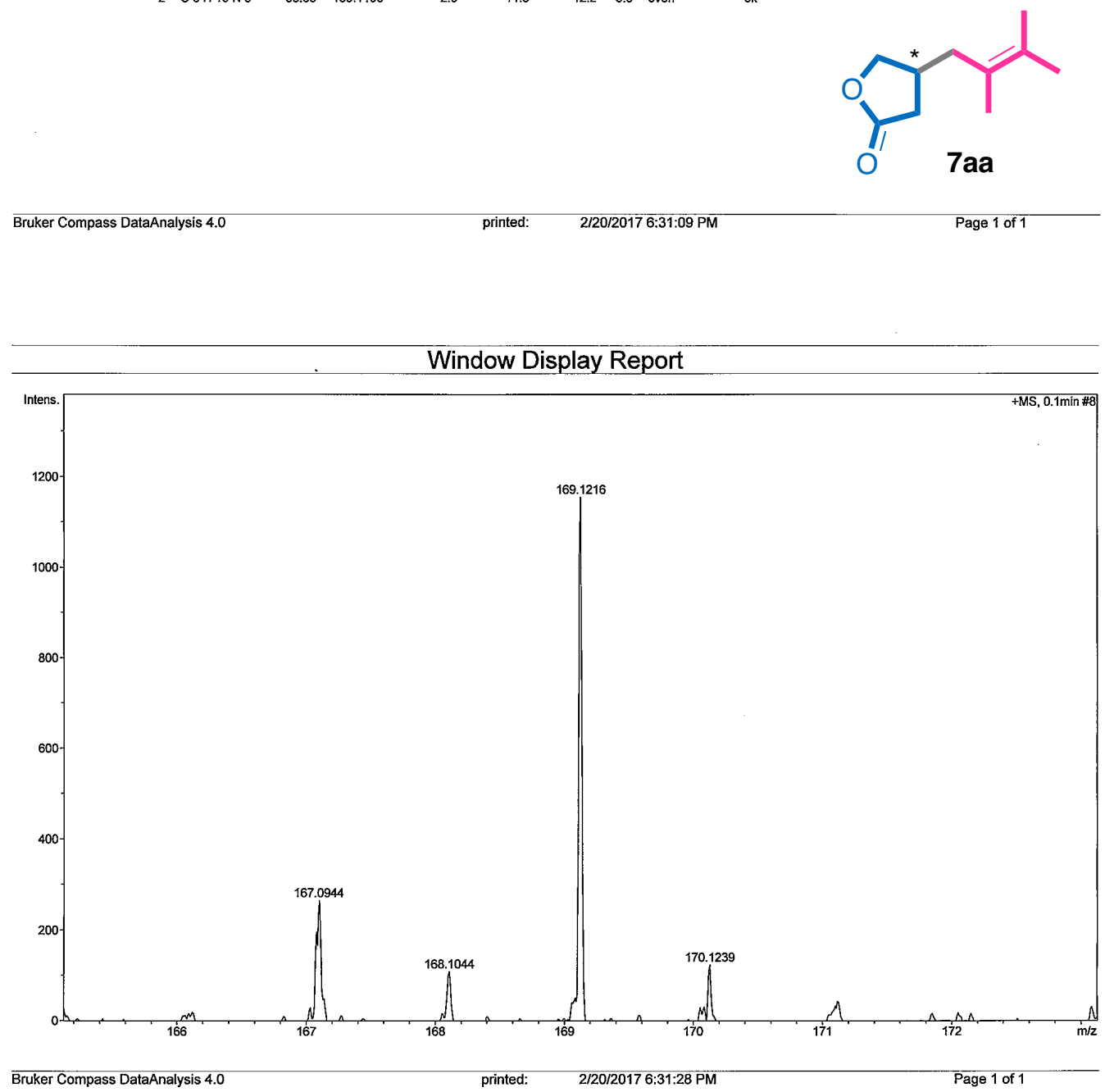

Figure S28-3. HRMS (APCI) of ent-4-(2,3-dimethylbu-2-en-1-yl)dihydrofuran-2(3H)-one (ent-7aa) 


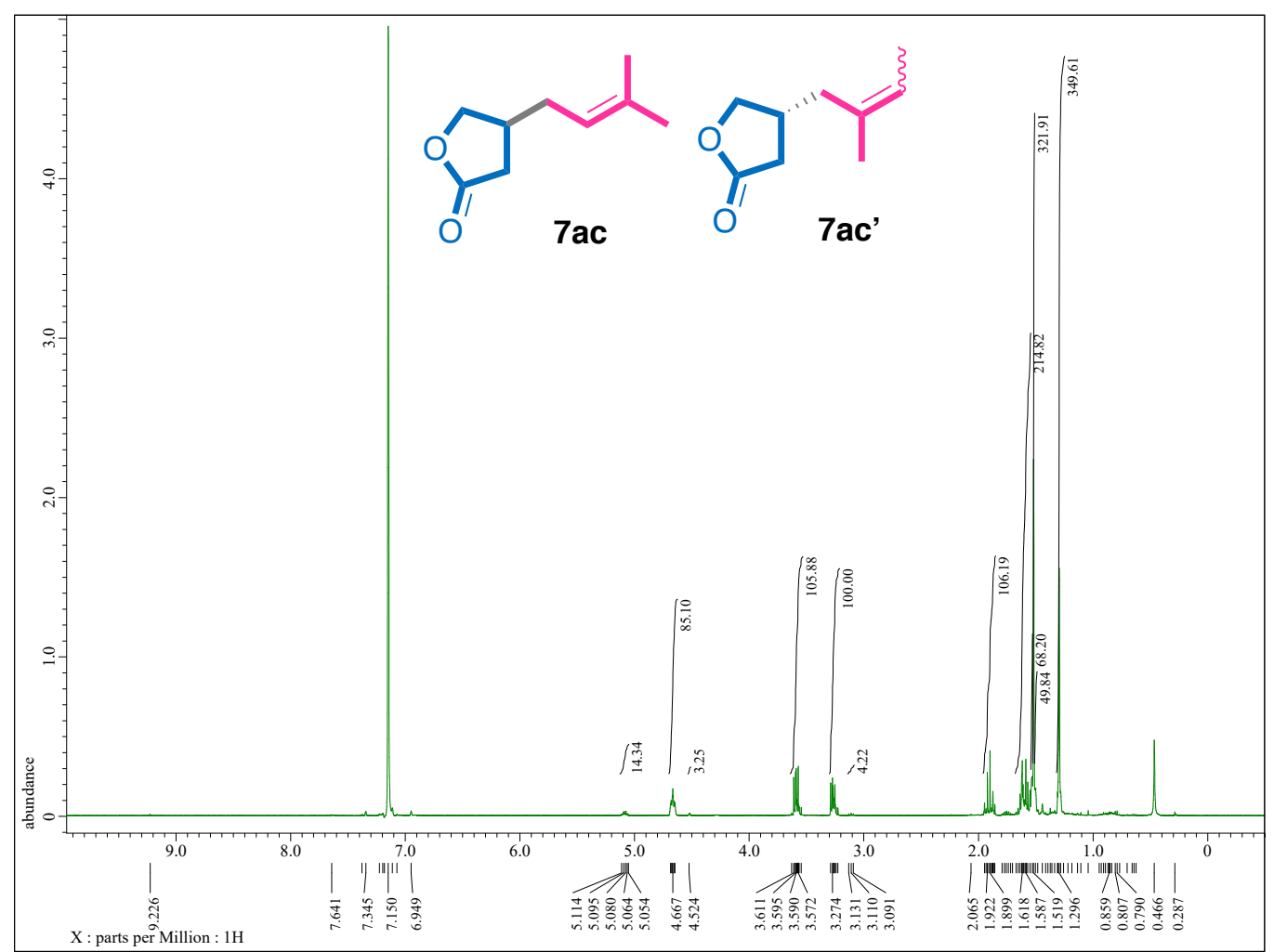

Figure S29-1. 'H NMR Spectrum of rac-4-(3-methylbut-2-en-1-yl)dihydrofuran-2(3H)-one (rac-7ac) rac4-(2-methylbut-2-en-1-yl)dihydrofuran-2(3H)-one (rac-7ac') (400 MHz, $\mathrm{C}_{6} \mathrm{D}_{6}$, r.t.).

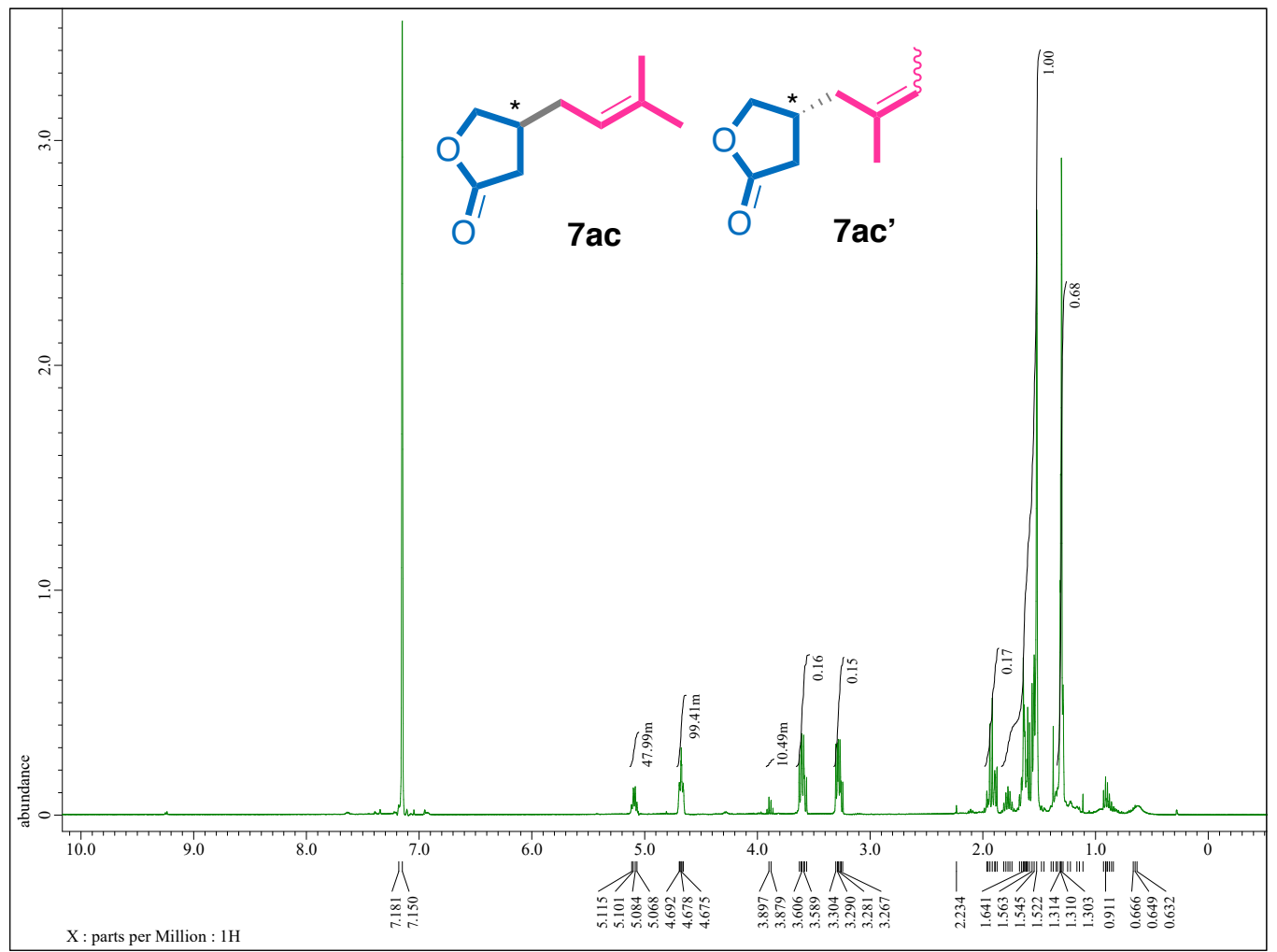

Figure S29-2. ' H NMR Spectrum of ent-4-(3-methylbut-2-en-1-yl)dihydrofuran-2(3H)-one (ent-7ac) rac4-(2-methylbut-2-en-1-yl)dihydrofuran-2(3H)-one (ent-7ac') (400 MHz, $\mathrm{C}_{6} \mathrm{D}_{6}$, r.t.). 


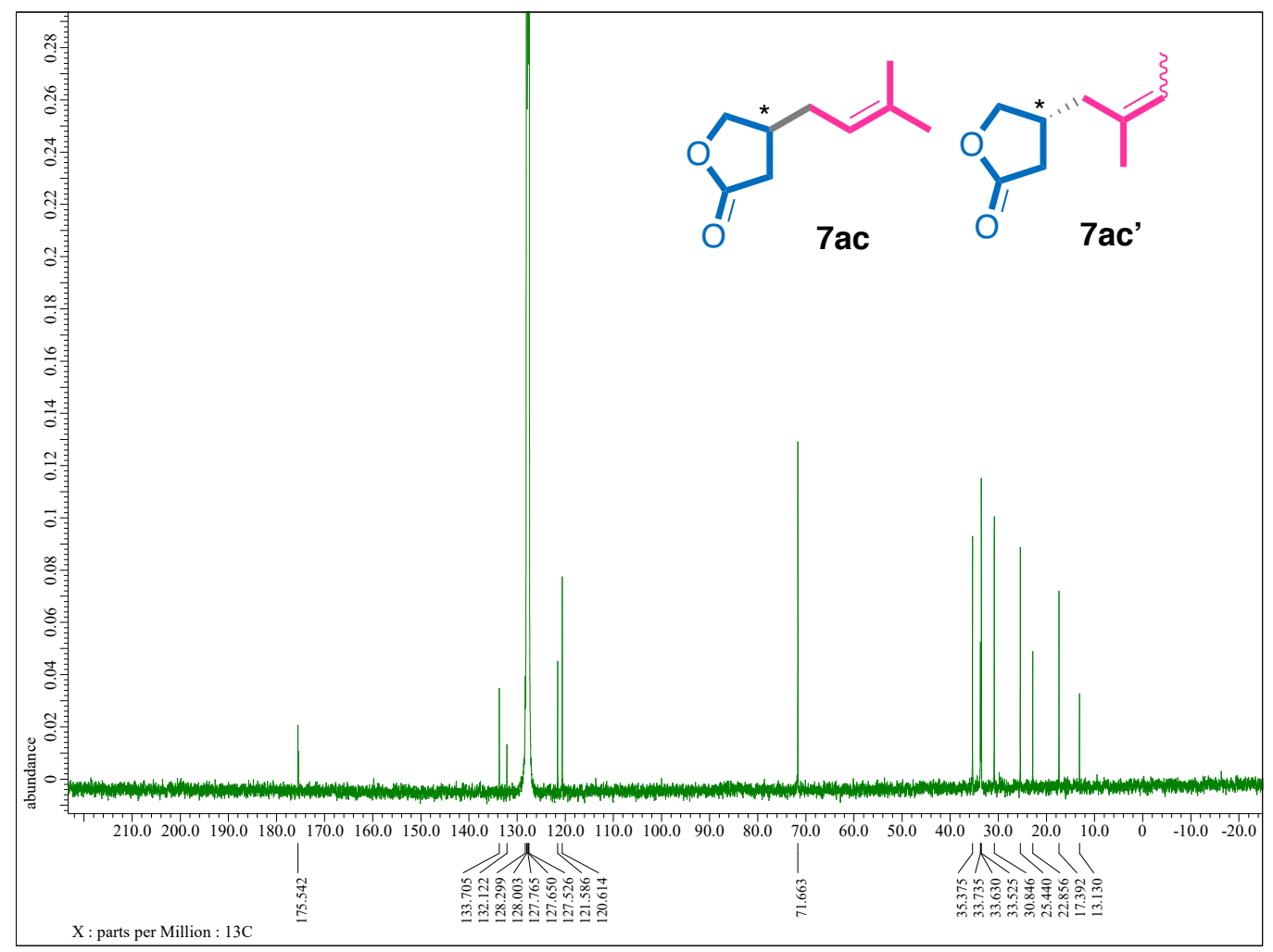

Figure S29-3. ${ }^{13} \mathrm{C}\left\{{ }^{1} \mathrm{H}\right\}$ NMR Spectrum of 4-(3-methylbut-2-en-1-yl)dihydrofuran-2(3H)-one (7ac) and 4(2-methylbut-2-en-1-yl)dihydrofuran-2(3H)-one (7ac') (100 MHz, $\mathrm{C}_{6} \mathrm{D}_{6}$, r.t.).

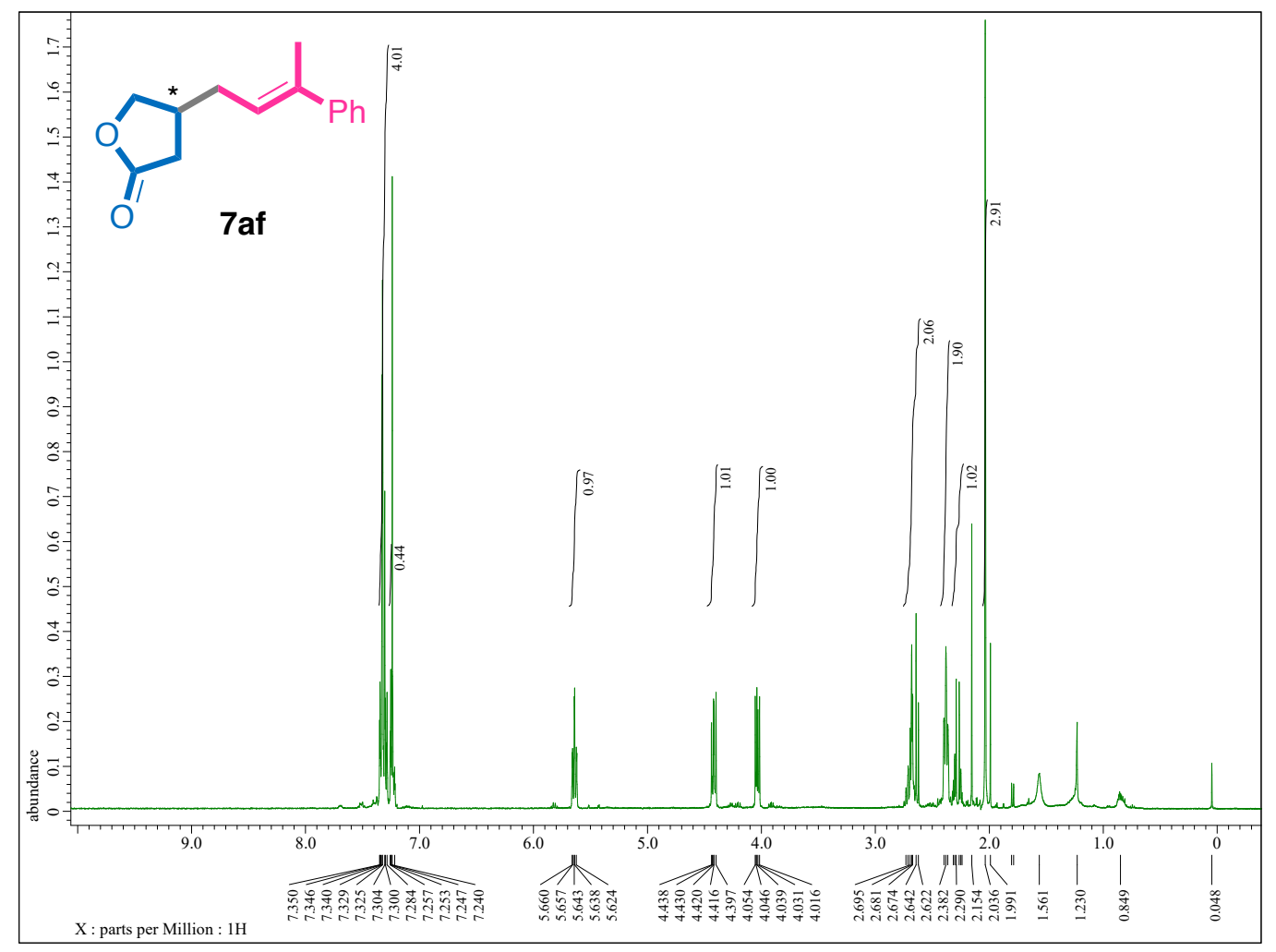

Figure S30-1. ' $\mathrm{H}$ NMR Spectrum of 4-(3-phenylbut-2-en-1-yl)dihydrofuran-2(3H)-one (7af) $(400 \mathrm{MHz}$, $\mathrm{CDCl}_{3}$, r.t.). 


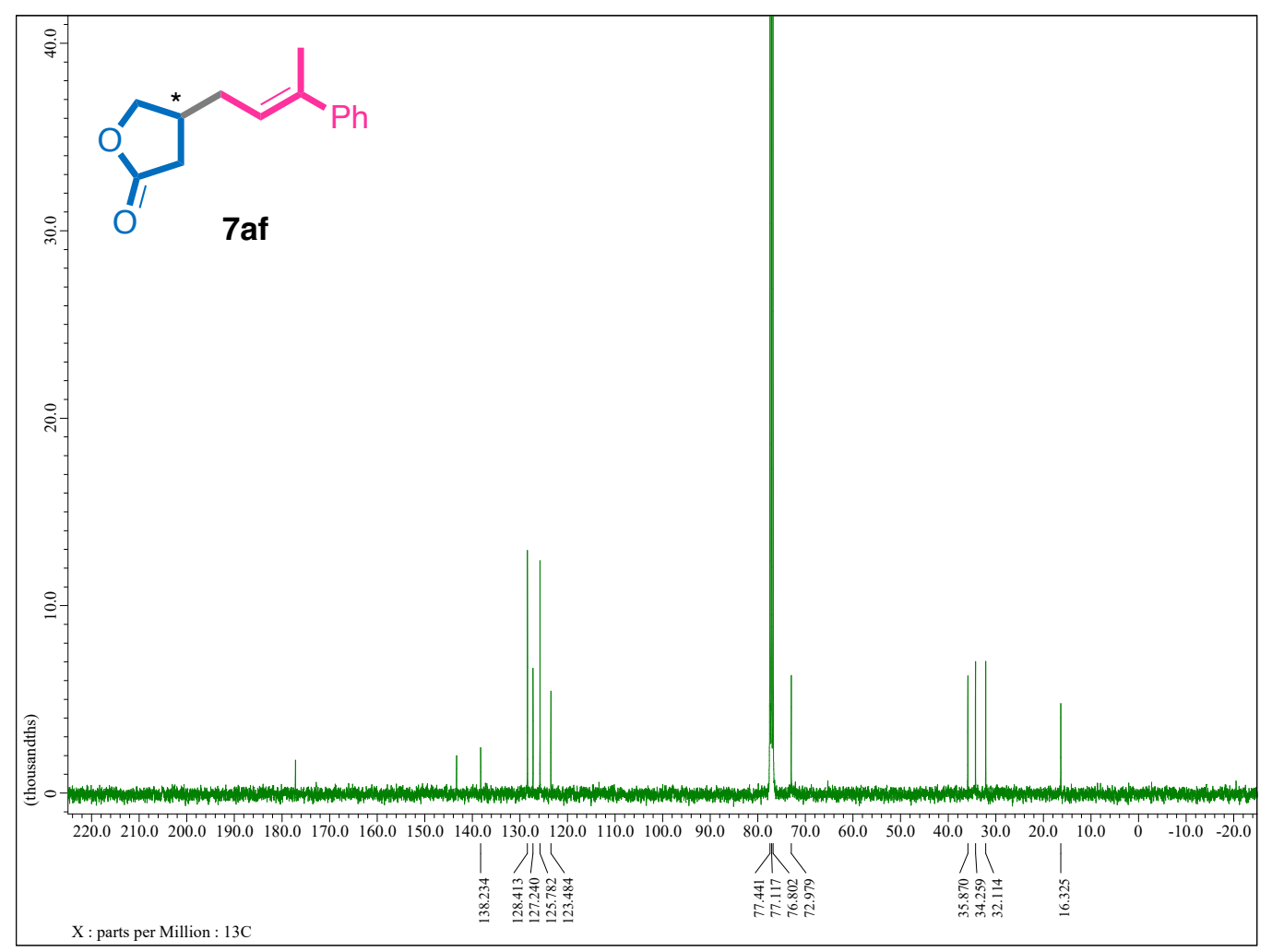

Figure S30-2. ${ }^{13} \mathrm{C}\left\{{ }^{1} \mathrm{H}\right\}$ NMR Spectrum of 4-(3-phenylbut-2-en-1-yl)dihydrofuran-2(3H)-one (7af) (100 $\mathrm{MHz}, \mathrm{CDCl}_{3}$, r.t.). 


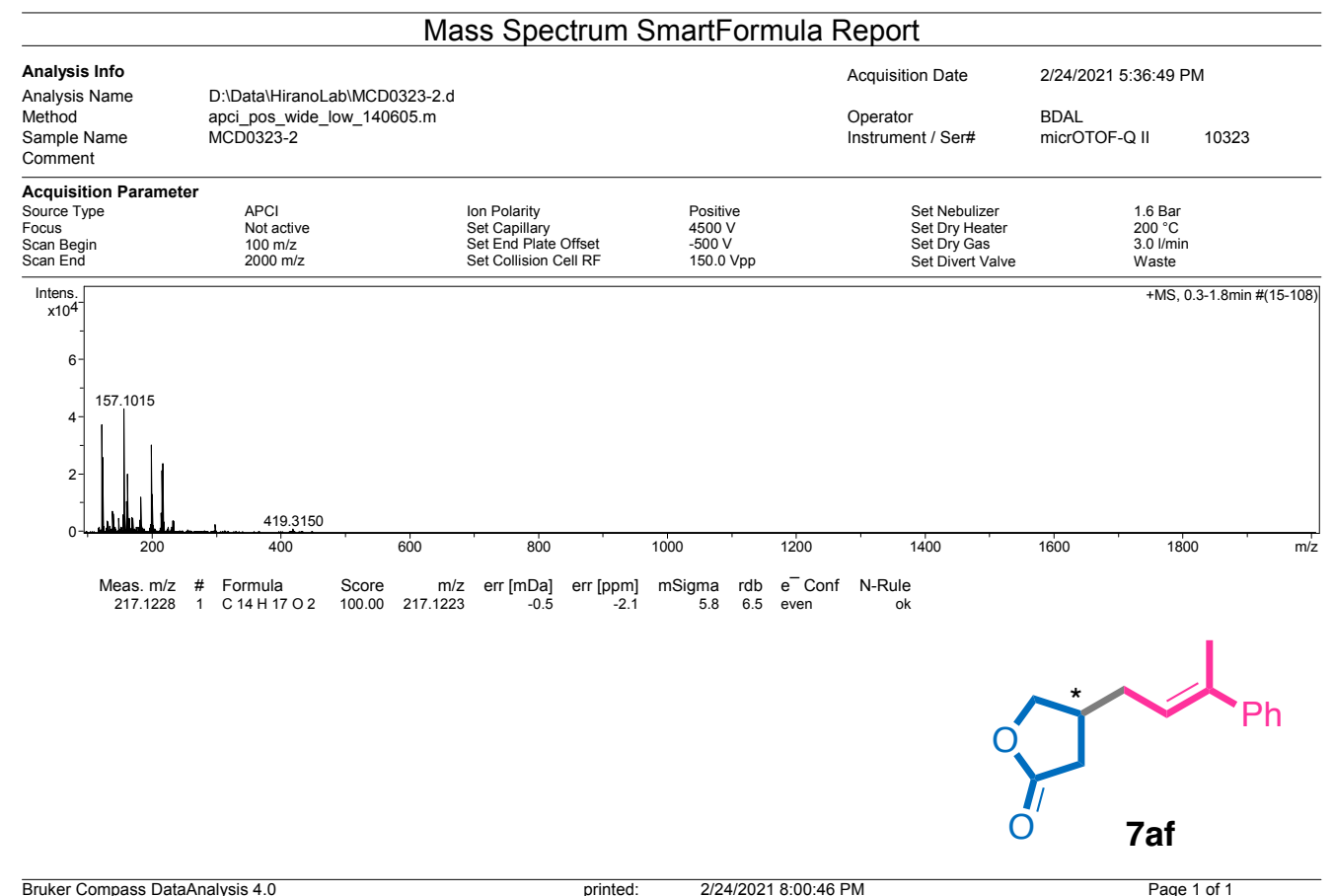

Bruker Compass DataAnalysis 4.0

printed: $\quad$ 2/24/2021 8:00:46 PM

Page 1 of 1

Window Display Report

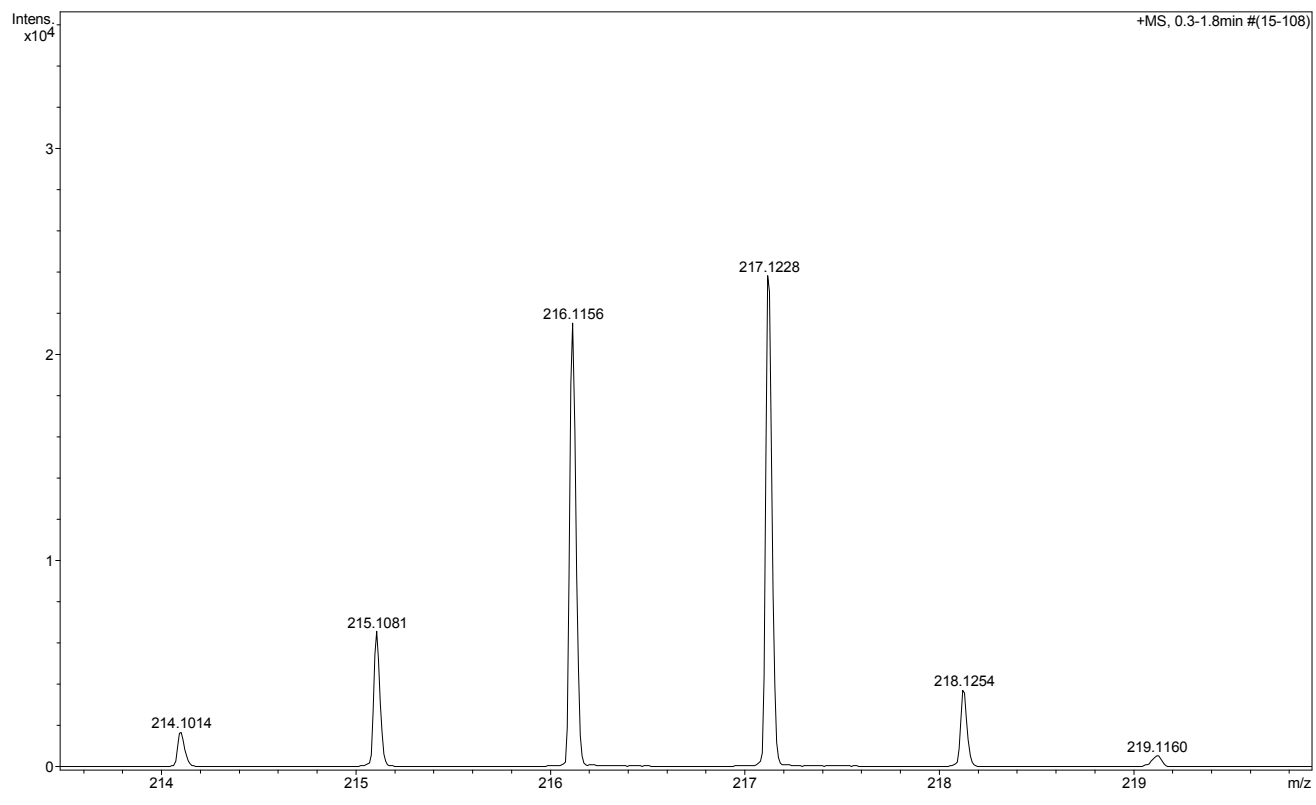

\begin{tabular}{lll}
\hline Bruker Compass DataAnalysis 4.0 & printed: & 2/24/2021 8:01:15 PM
\end{tabular}

Figure S30-3. HRMS (APCI) Spectrum of 4-(3-phenylbut-2-en-1-yl)dihydrofuran-2(3H)-one (7af). 


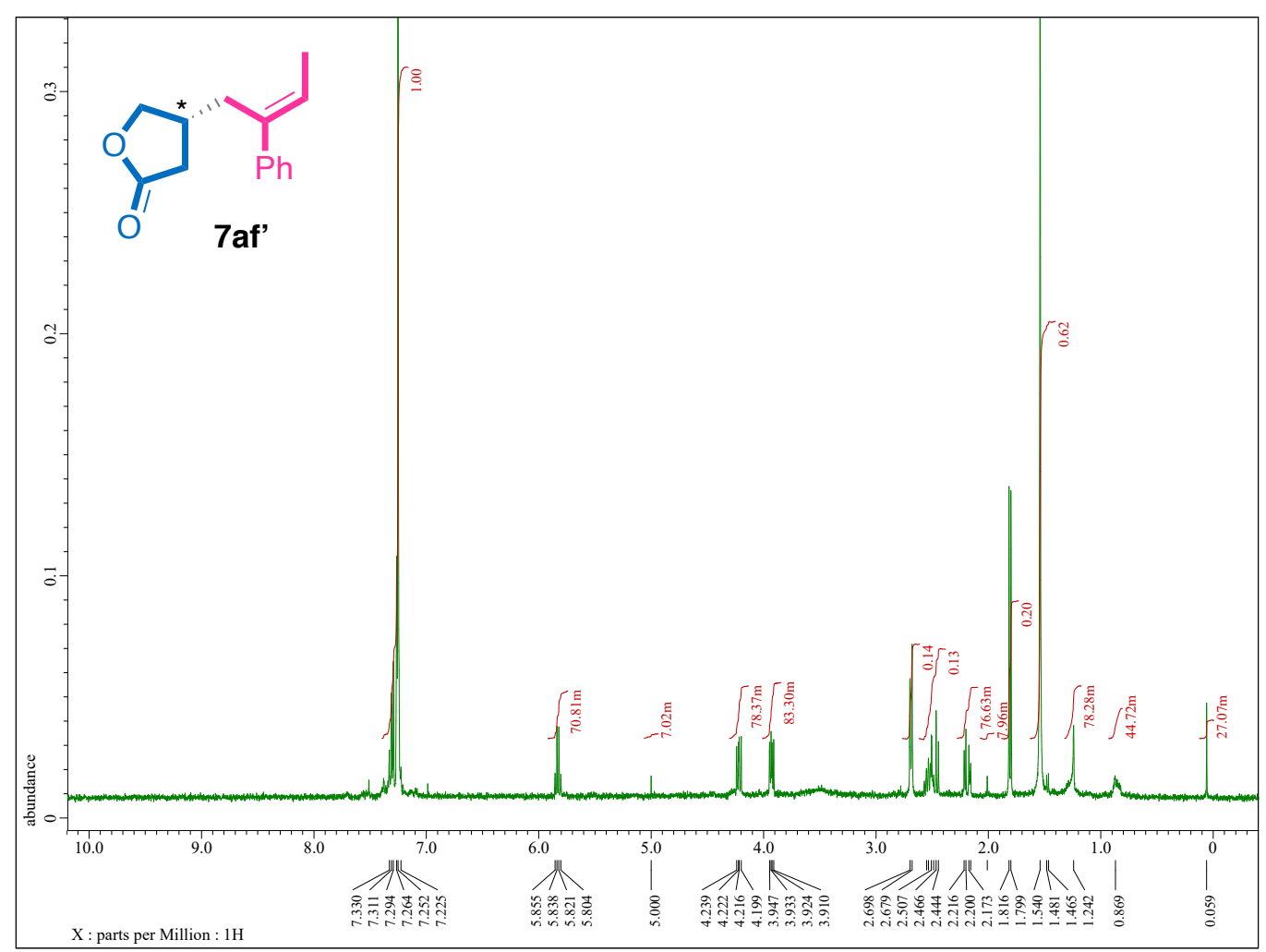

Figure S31-1. ${ }^{1} \mathrm{H}$ NMR Spectrum of 4-(2-phenylbut-2-en-1-yl)dihydrofuran-2(3H)-one (7af') $(400 \mathrm{MHz}$, $\mathrm{CDCl}_{3}$, r.t.).

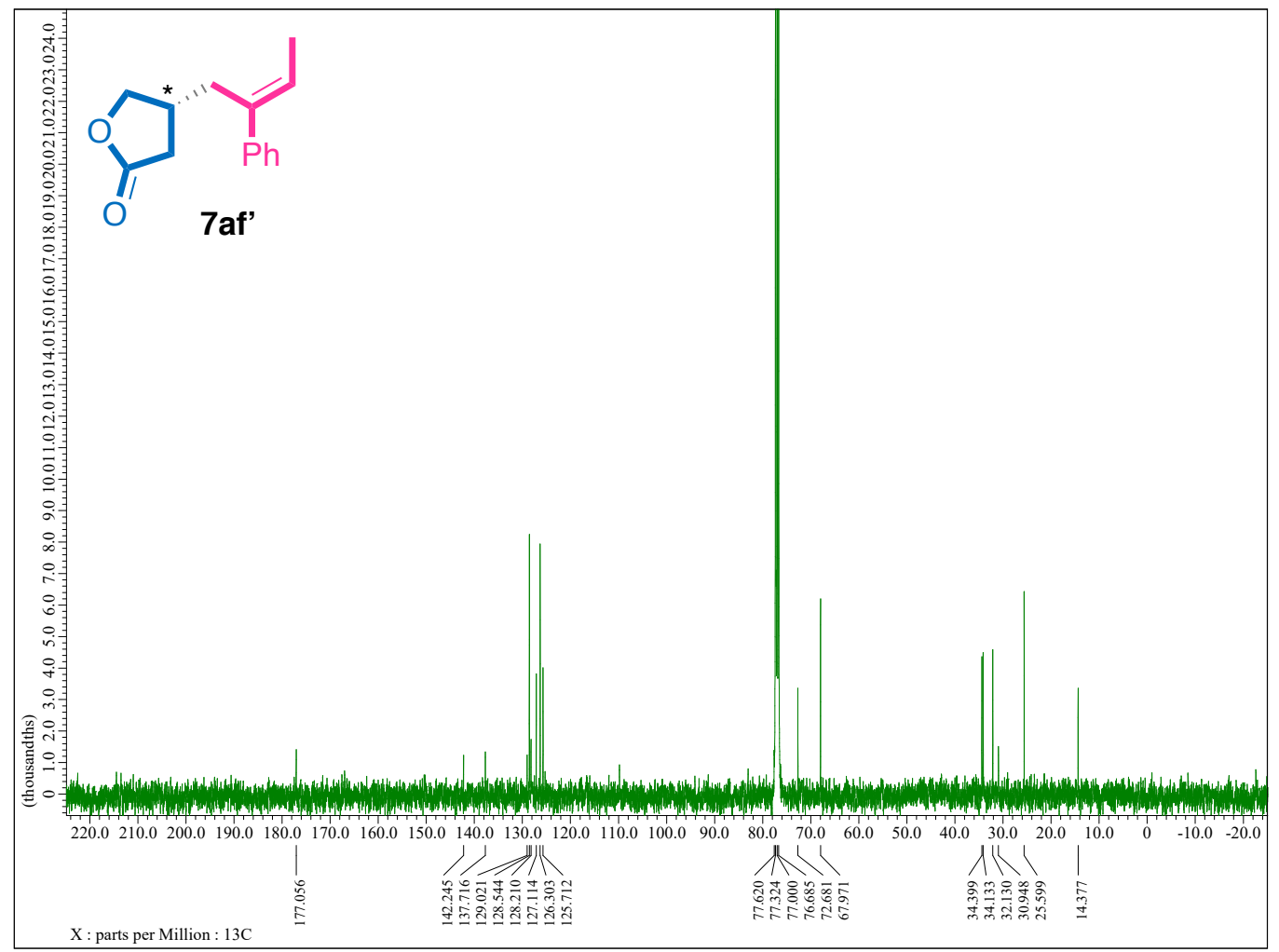

Figure S31-2. ${ }^{13} \mathrm{C}\left\{{ }^{1} \mathrm{H}\right\}$ NMR Spectrum of 4-(2-phenylbut-2-en-1-yl)dihydrofuran-2(3H)-one (7af') (100 $\mathrm{MHz}, \mathrm{CDCl}_{3}$, r.t.). 


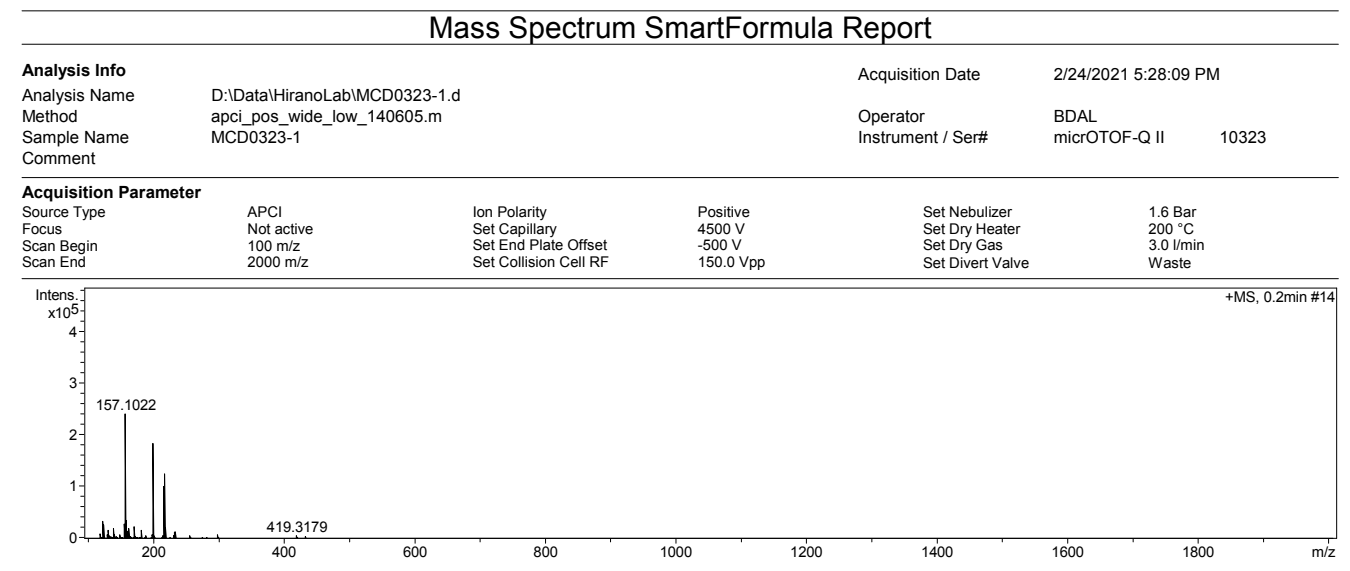

$\begin{array}{rrlrrrrrrrr}\text { Meas. } \mathrm{m} / \mathrm{z} & \# & \text { Formula } & \text { Score } & \mathrm{m} / \mathrm{z} & \text { err [mDa] } & \text { err [ppm] } & \text { mSigma } & \text { rdb } & \mathrm{e}^{-} \text {Conf } & \mathrm{N}-\text { Rule } \\ 217.1231 & 1 & \mathrm{C} 14 \mathrm{H} 17 \mathrm{O} 2 & 100.00 & 217.1223 & -0.8 & -3.7 & 8.5 & 6.5 & \text { even } & \text { ok }\end{array}$

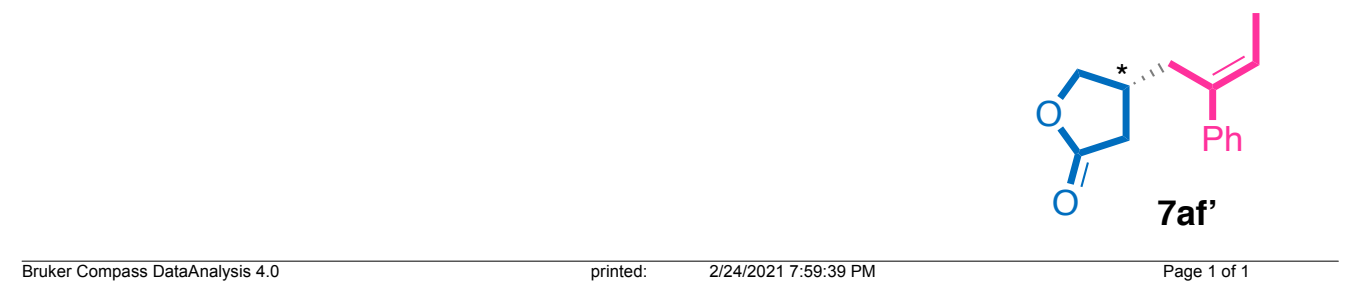

Window Display Report

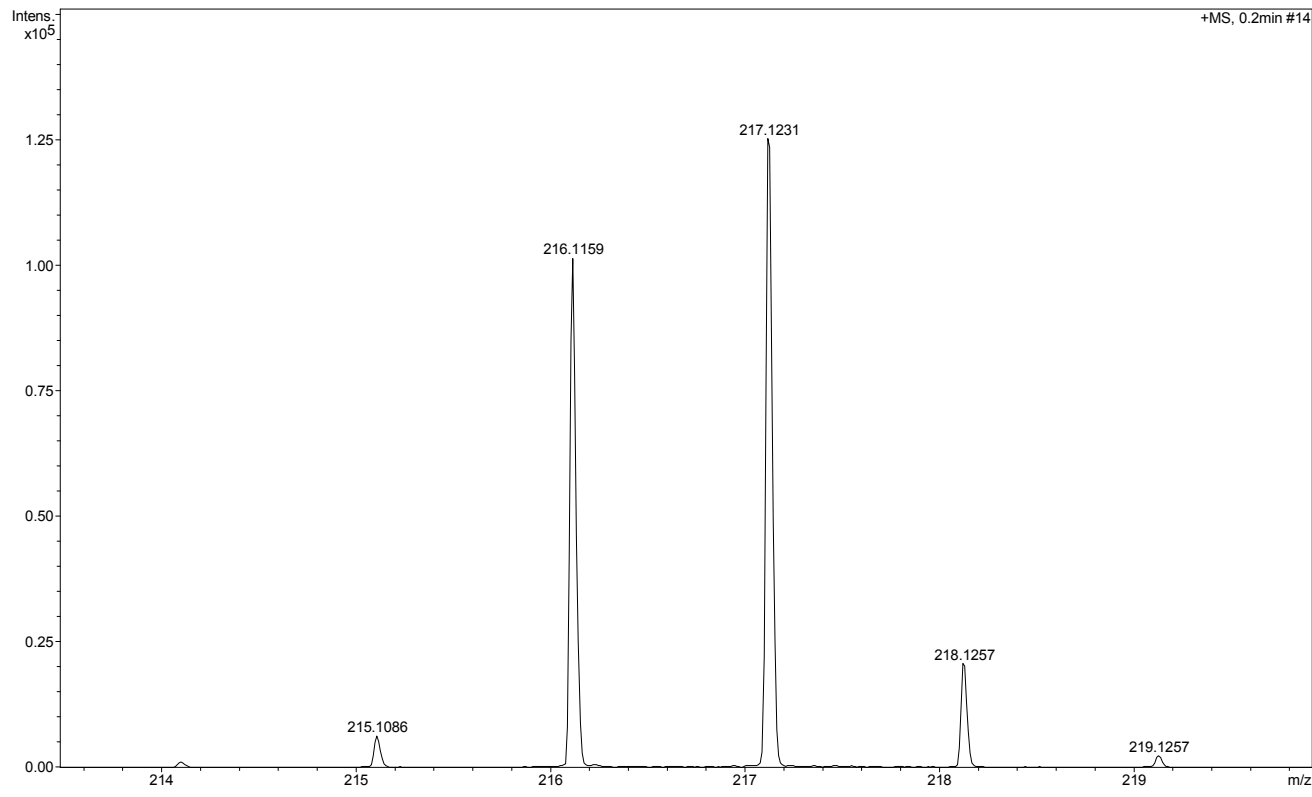

Bruker Compass DataAnalysis $4.0 \quad$ printed: $\quad$ 2/24/2021 8:00:23 PM

Page 1 of 1

Figure S31-3. HRMS (APCI) Spectrum of 4-(2-phenylbut-2-en-1-yl)dihydrofuran-2(3H)-one (7af'). 


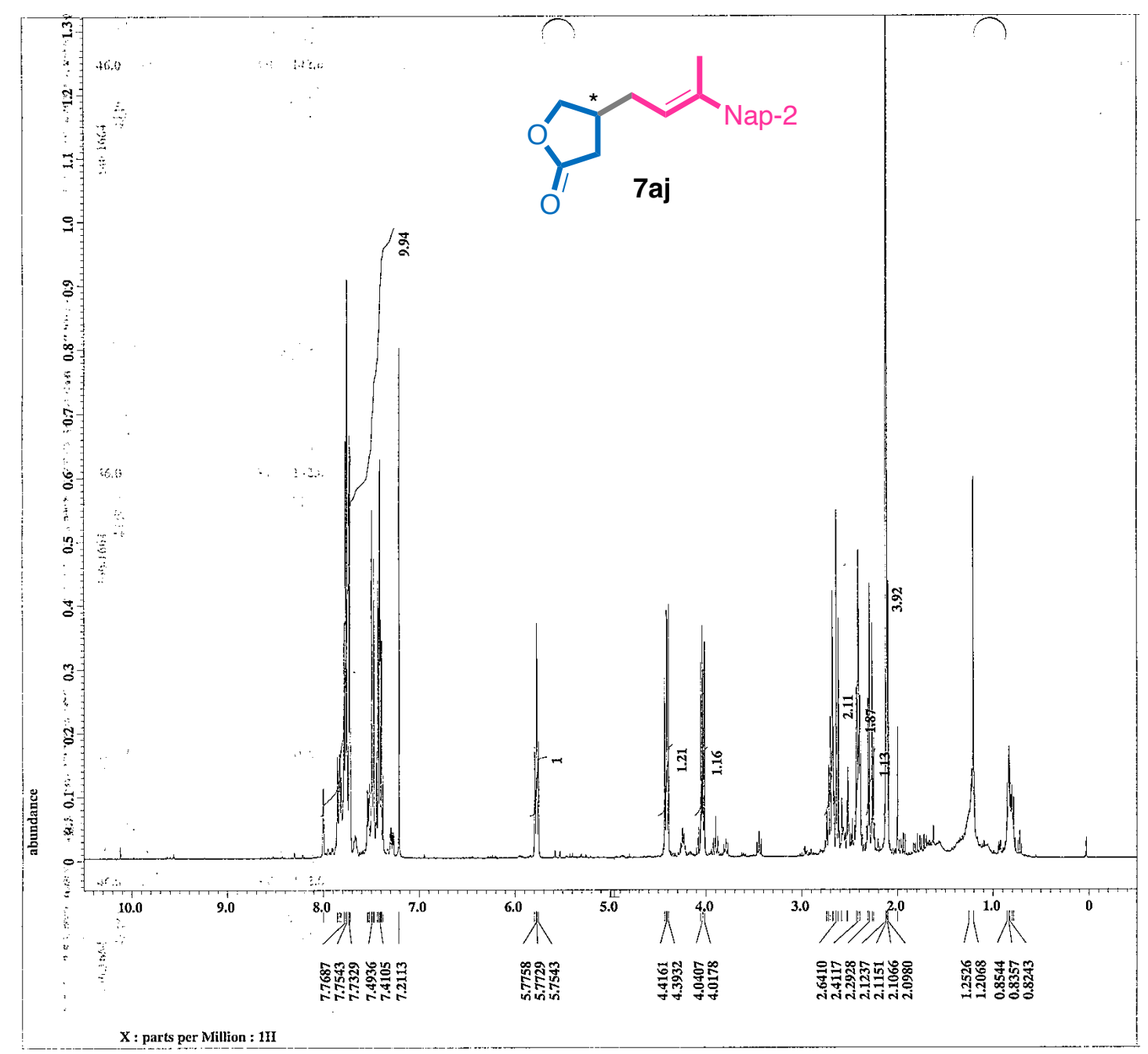

Figure S32-1. ${ }^{1} \mathrm{H}$ NMR Spectrum of 4-(3-2-naphthylbut-2-en-1-yl)dihydrofuran-2(3H)-one (7aj) (400 $\mathrm{MHz}, \mathrm{CDCl}_{3}$, r.t.). 


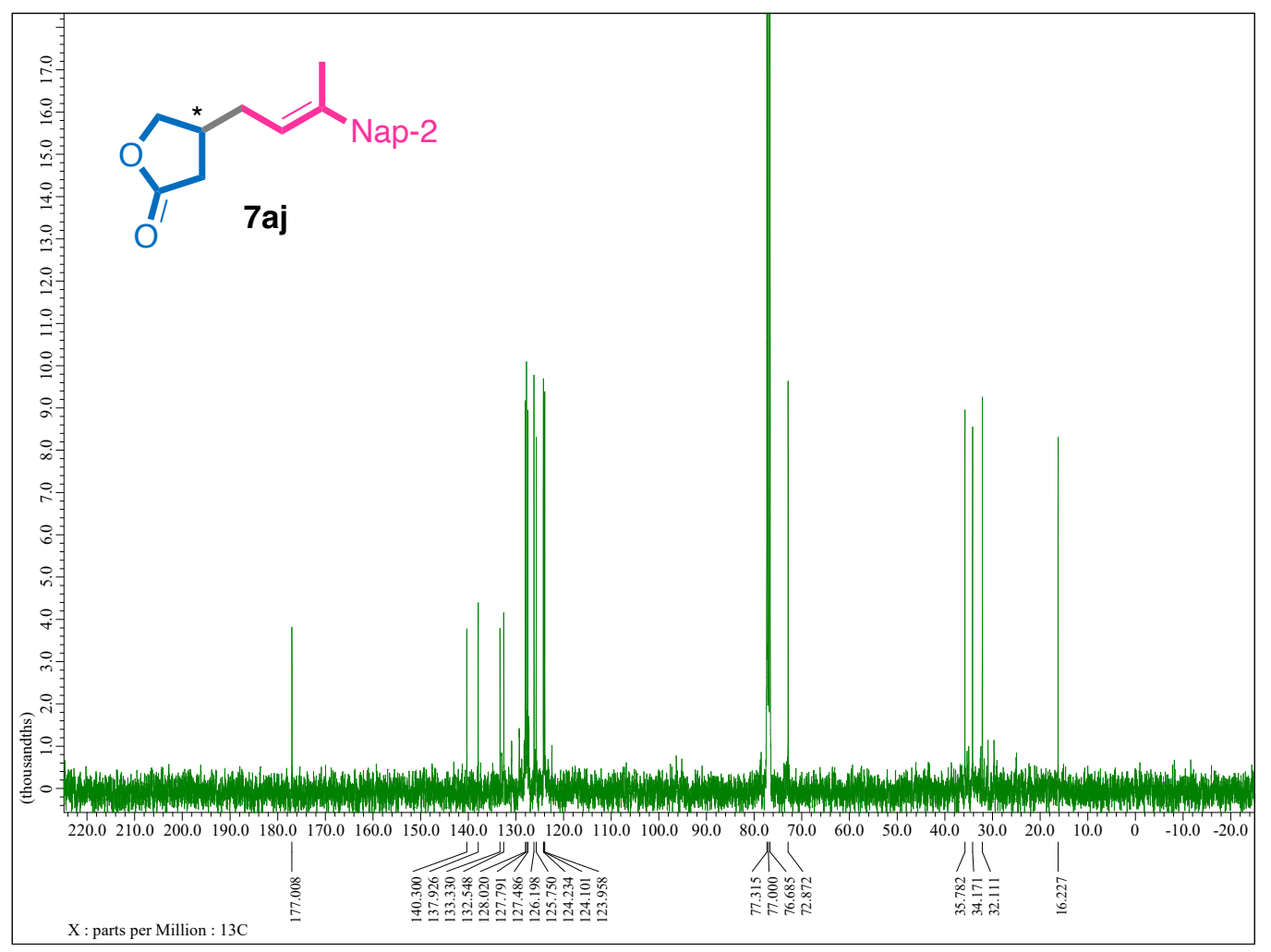

Figure S32-2. ${ }^{13} \mathrm{C}\left\{{ }^{1} \mathrm{H}\right\}$ NMR Spectrum of 4-(3-2-naphthylbut-2-en-1-yl)dihydrofuran-2(3H)-one (7aj) (100 $\mathrm{MHz}, \mathrm{CDCl}_{3}$, r.t.).

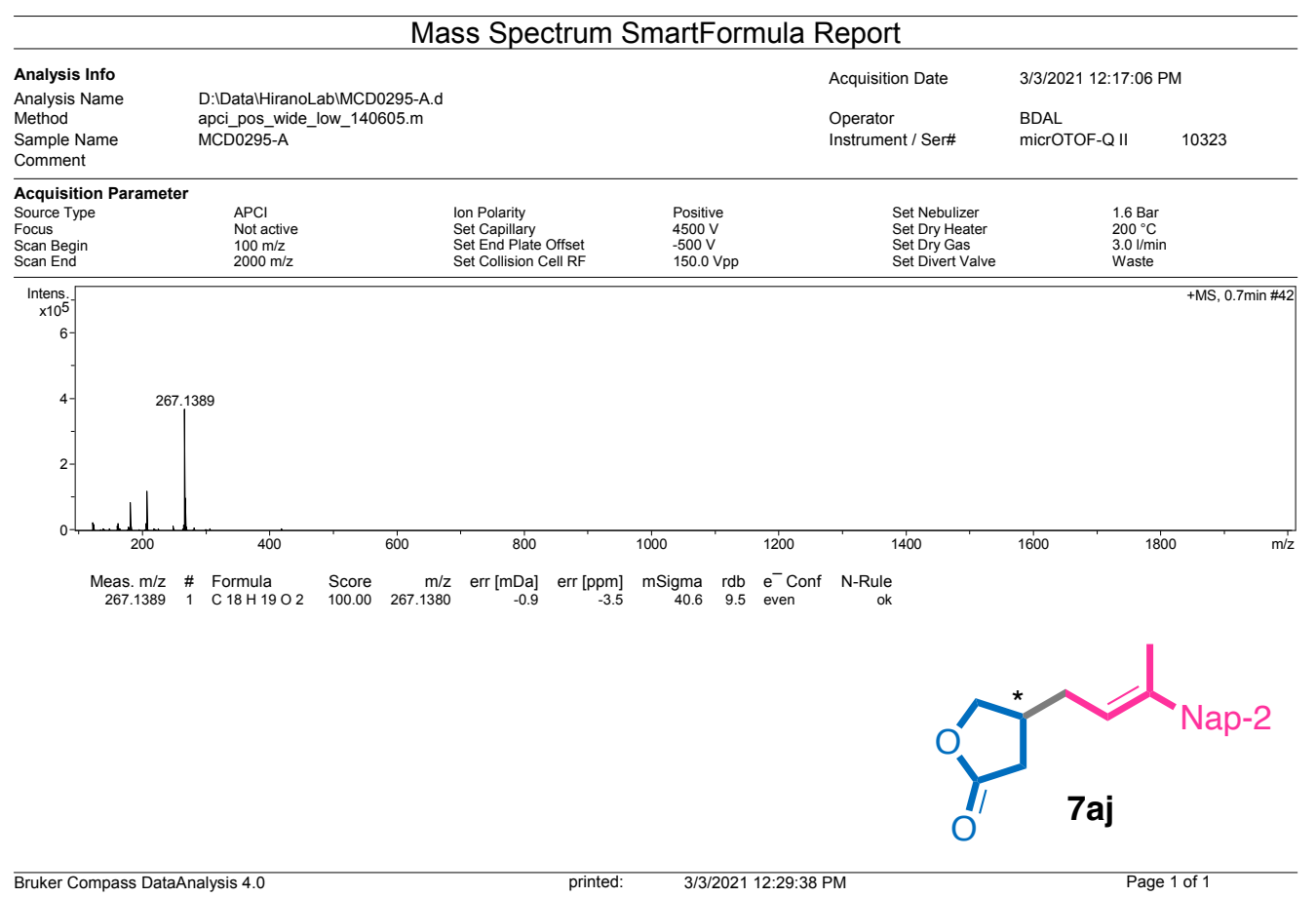

Figure S32-3. HRMS (APCI) of 4-(3-2-naphthylbut-2-en-1-yl)dihydrofuran-2(3H)-one (7aj) 


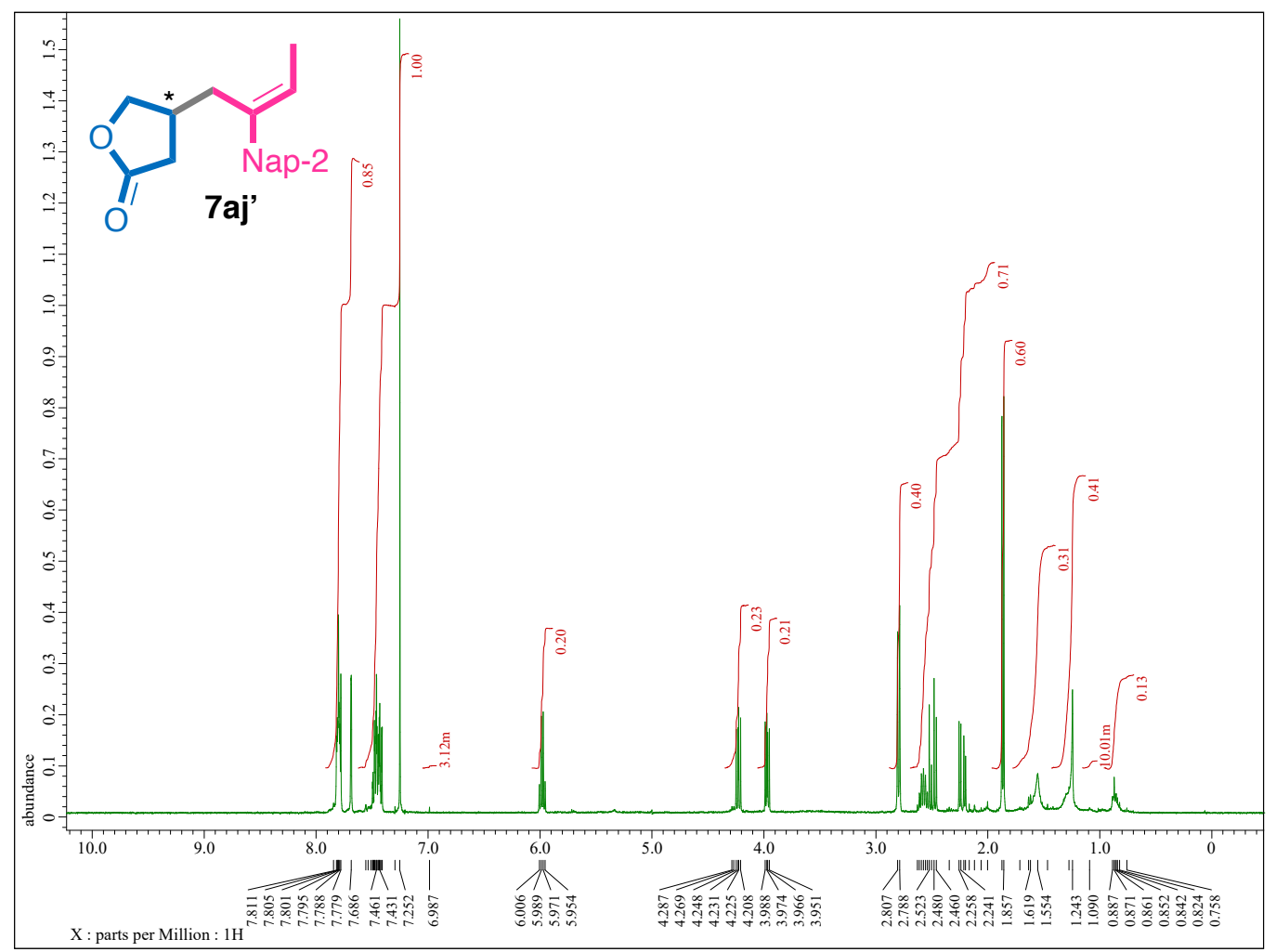

Figure S33-1. ${ }^{1} \mathrm{H}$ NMR Spectrum of 4-(2-2-naphthylbut-2-en-1-yl)dihydrofuran-2(3H)-one (7aj') (400 $\mathrm{MHz}, \mathrm{CDCl}_{3}$, r.t.).

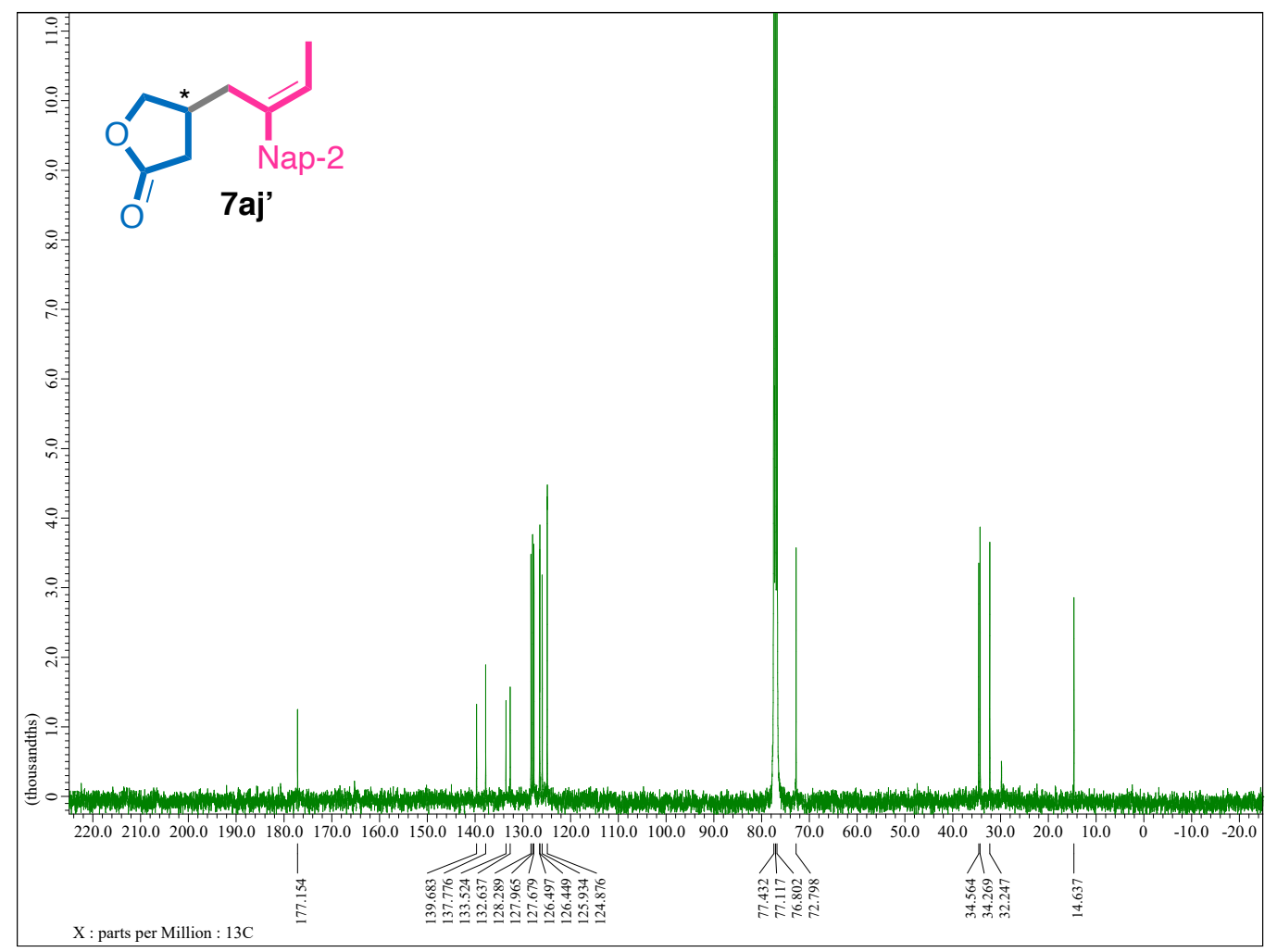

Figure S33-2. ${ }^{13} \mathrm{C}\left\{{ }^{1} \mathrm{H}\right\}$ NMR Spectrum of 4-(2-2-naphthylbut-2-en-1-yl)dihydrofuran-2(3H)-one (7aj') $\left(100 \mathrm{MHz}, \mathrm{CDCl}_{3}\right.$, r.t.). 


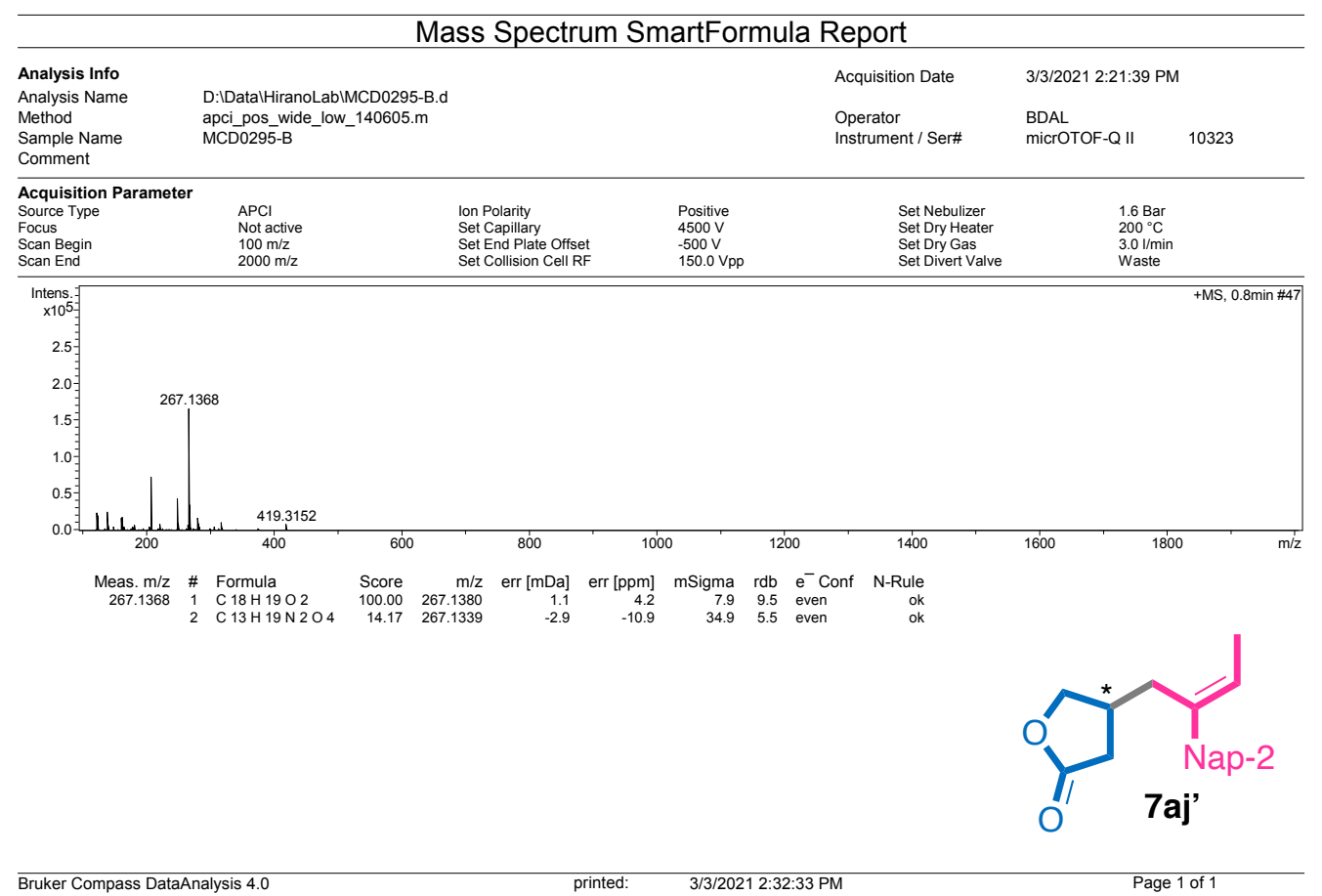

Figure S33-2. HRMS (APCI) of 4-(2-2-naphthylbut-2-en-1-yl)dihydrofuran-2(3H)-one (7aj')

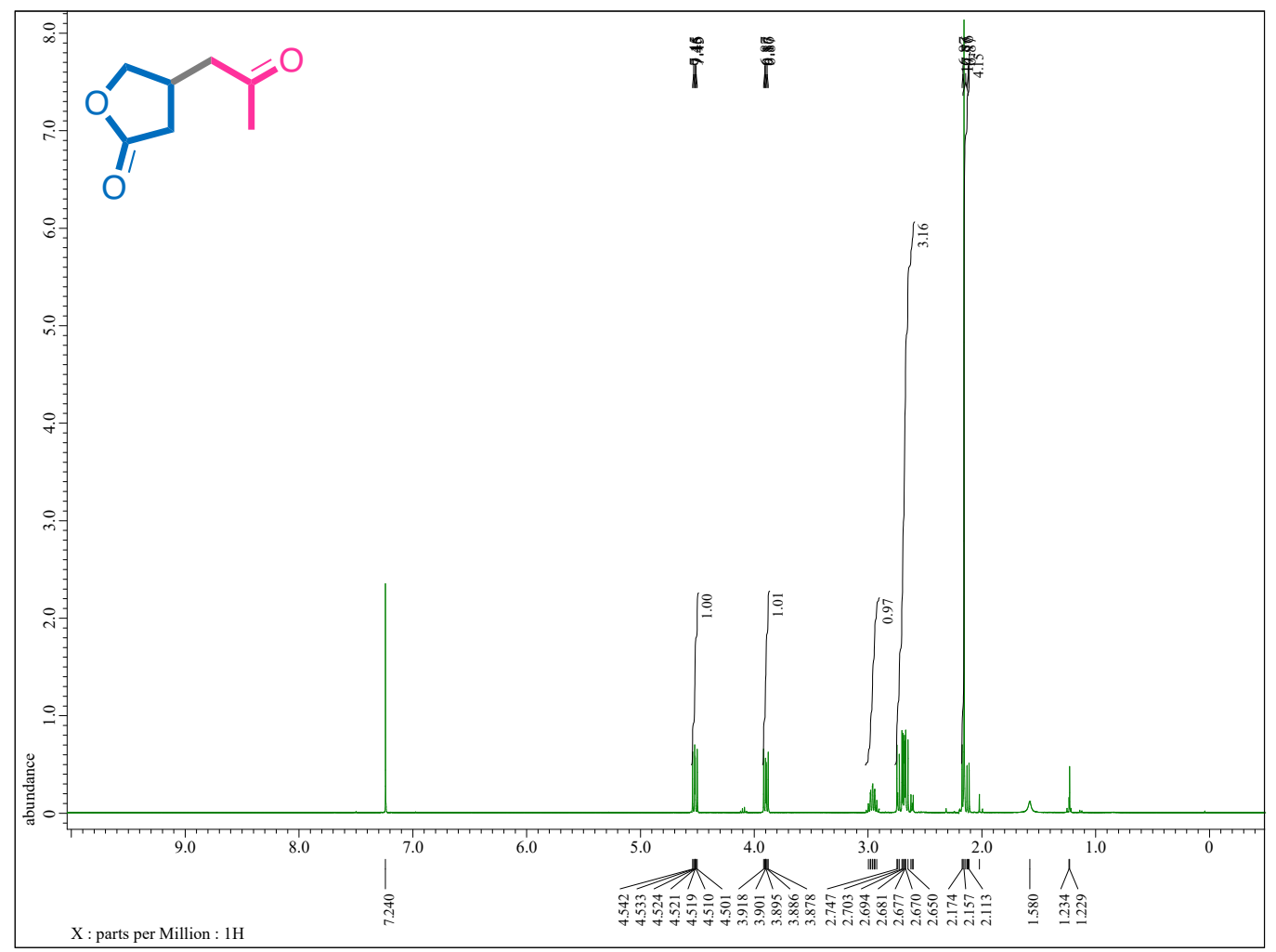

Figure S34-1. ${ }^{1} \mathrm{H}$ NMR Spectrum of 4-(2-oxopropyl)dihydrofuran-2(3H)-one (ent-8aa) $\left(400 \mathrm{MHz}, \mathrm{CDCl}_{3}\right.$, r.t.). 


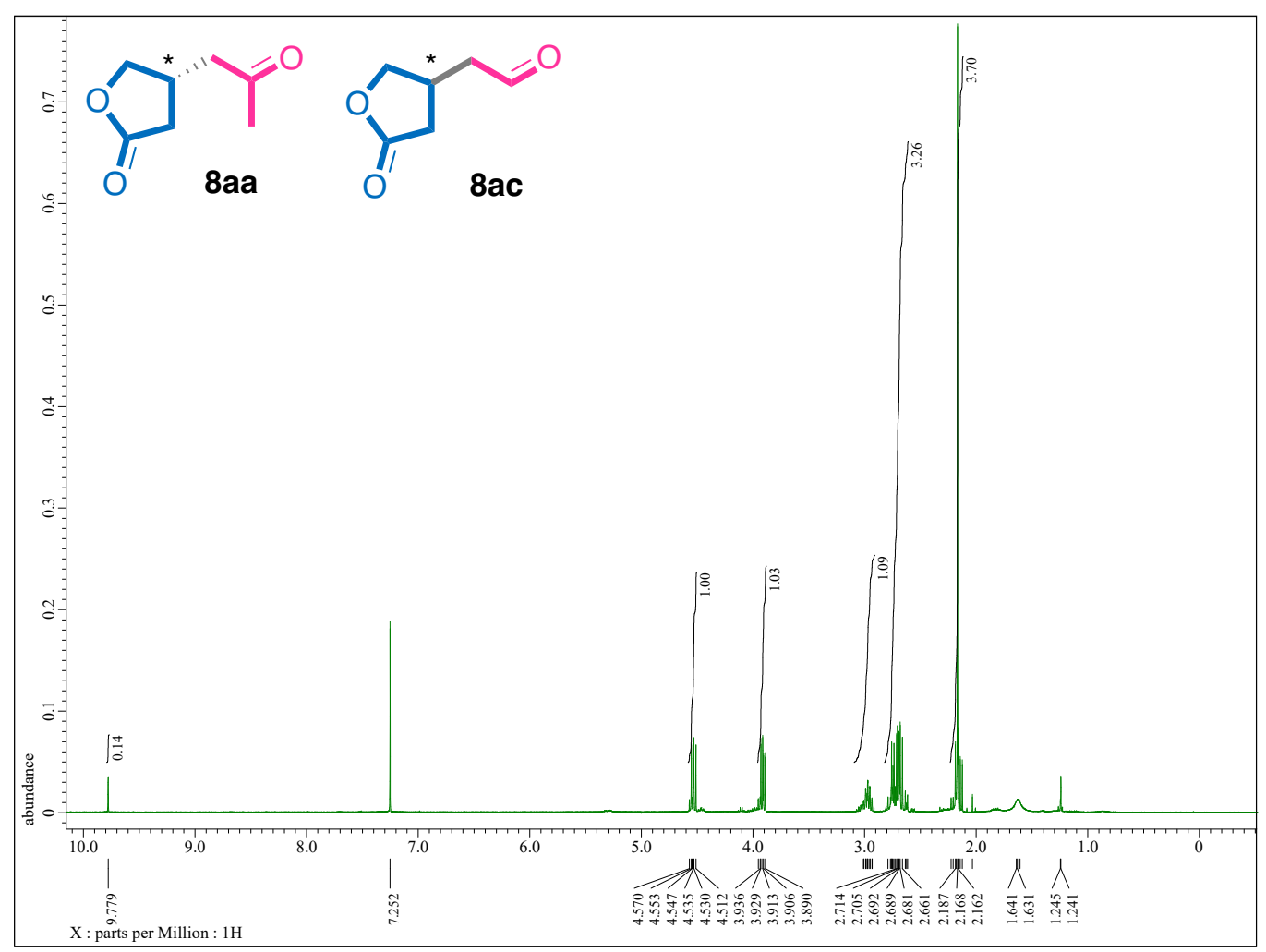

Figure S34-2. ${ }^{~} \mathrm{H}$ NMR Spectrum of 4-(2-oxopropyl)dihydrofuran-2(3H)-one (ent-8aa) and 2-(5oxotetrahydrofuran-3-yl)acetaldehyde (ent-8ac) $\left(400 \mathrm{MHz}, \mathrm{CDCl}_{3}\right.$, r.t.).

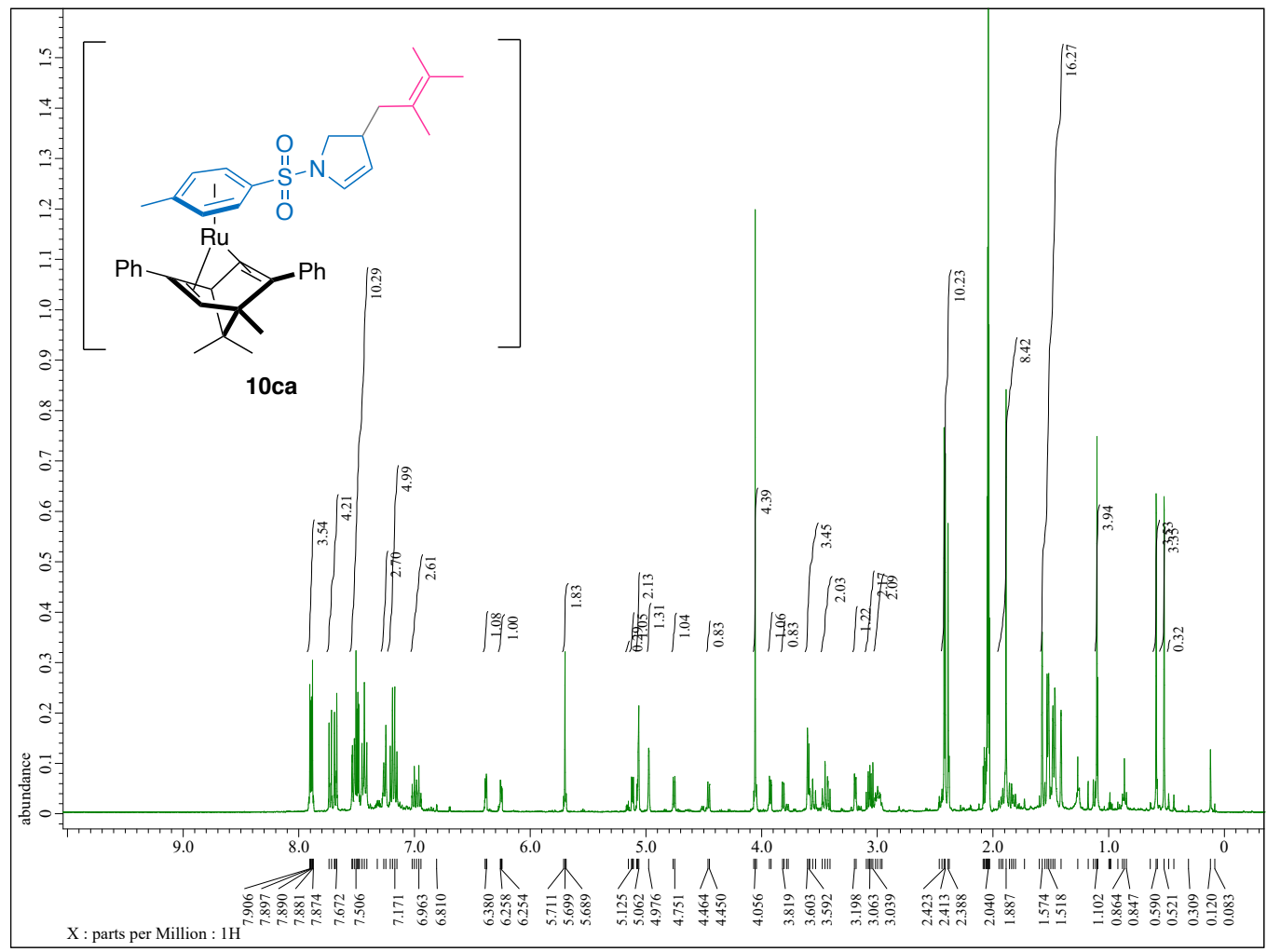

Figure S35-1. ${ }^{1} \mathrm{H}$ NMR Spectrum of [Ru\{ $\eta^{6}$-3-(2,3-dimethylbut-2,3-en-1-yl)-1-tosyl-2,3-dihydro-1Hpyrrole $\left\{\eta^{4}-(1 S, 4 S)-\mathrm{Ph}_{2}-\right.$ bhd $\left.d^{*}\right]$ (10ca) $\left(400 \mathrm{MHz}\right.$, acetone- $d_{6}$, r.t.). 


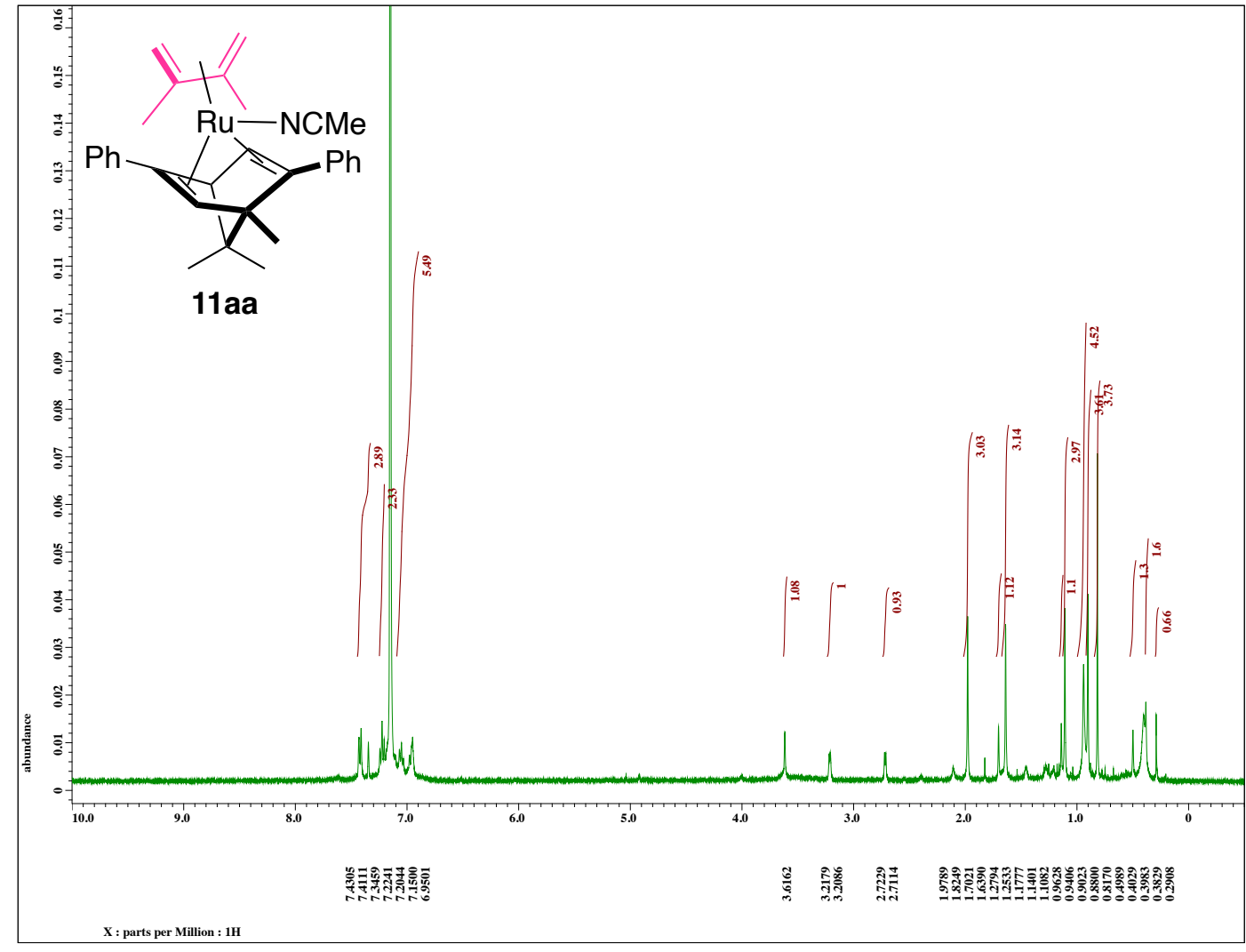

Figure S36-1. ' $\mathrm{H}$ NMR spectrum of $\left[\mathrm{Ru}\left(\right.\right.$ cisoid- $\eta^{4}-2,3-$ dimethylbutdiene) $\left.\left\{\eta^{4}-(1 S, 4 S)-\mathrm{Ph}_{2}-\mathrm{bhd}^{*}\right\}(\mathrm{NCMe})\right]$ (11a) $\left(400 \mathrm{MHz}, \mathrm{C}_{6} \mathrm{D}_{6}\right.$, r.t.). 


\section{Chromatograms}

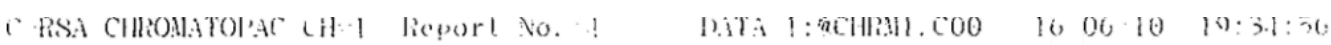
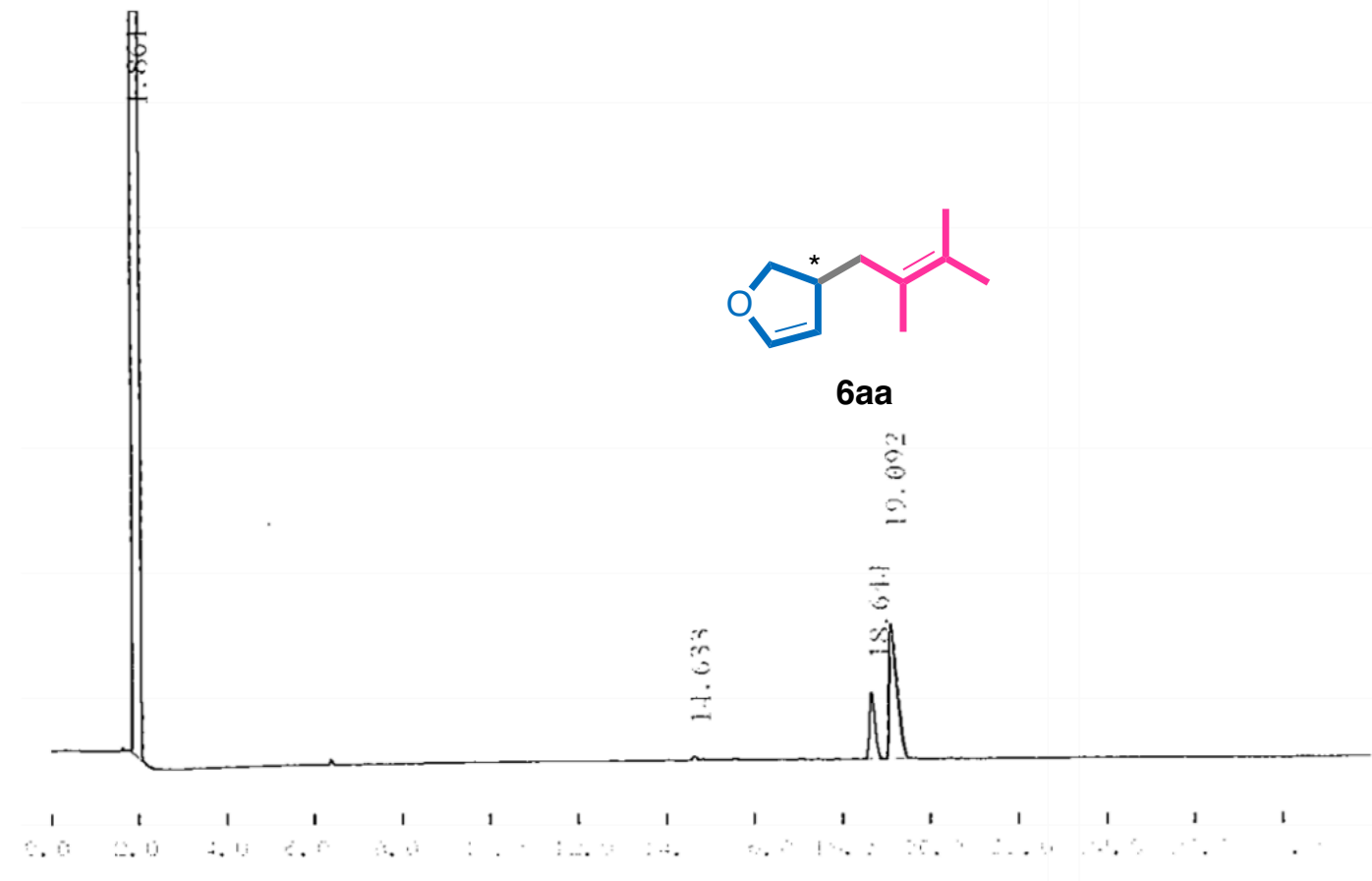

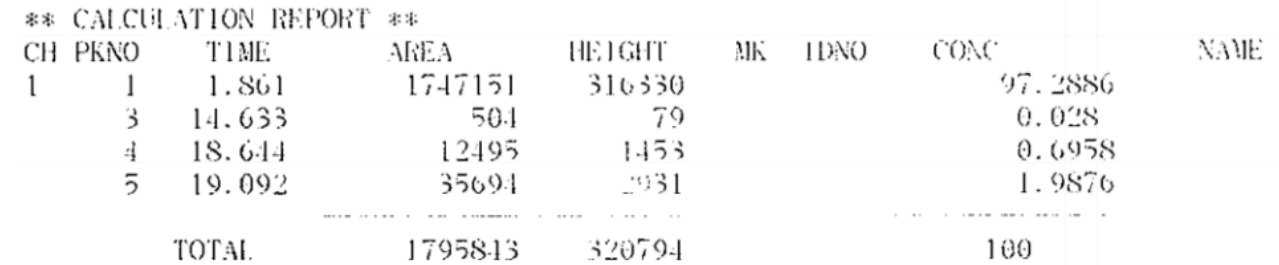

ent-6aa: $\mathrm{RT}=18.644,19.092 \mathrm{~min}$.

ee $=(35694-12495) /(35694+12495)=48 \%$ ee $(S)$

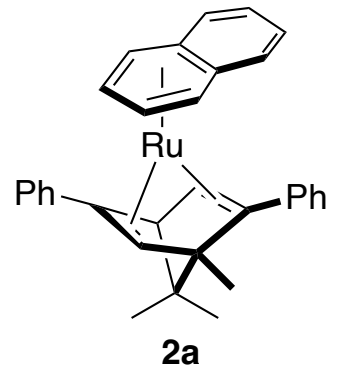

Figure S37-1. CSP-GC Chromatogram of ent-6aa produced by the catalysis using $2 \mathrm{a}(10 \mathrm{~mol} \%)$ at $30{ }^{\circ} \mathrm{C}$ in acetone. Conditions: Column: Rt- $\beta$ DEXsm $(0.25 \mathrm{mmf}$ x $30 \mathrm{~m})$, oven temp: $90^{\circ} \mathrm{C}$, linear velosity: $40 \mathrm{~cm}$ $\mathrm{s}^{-1}$. 

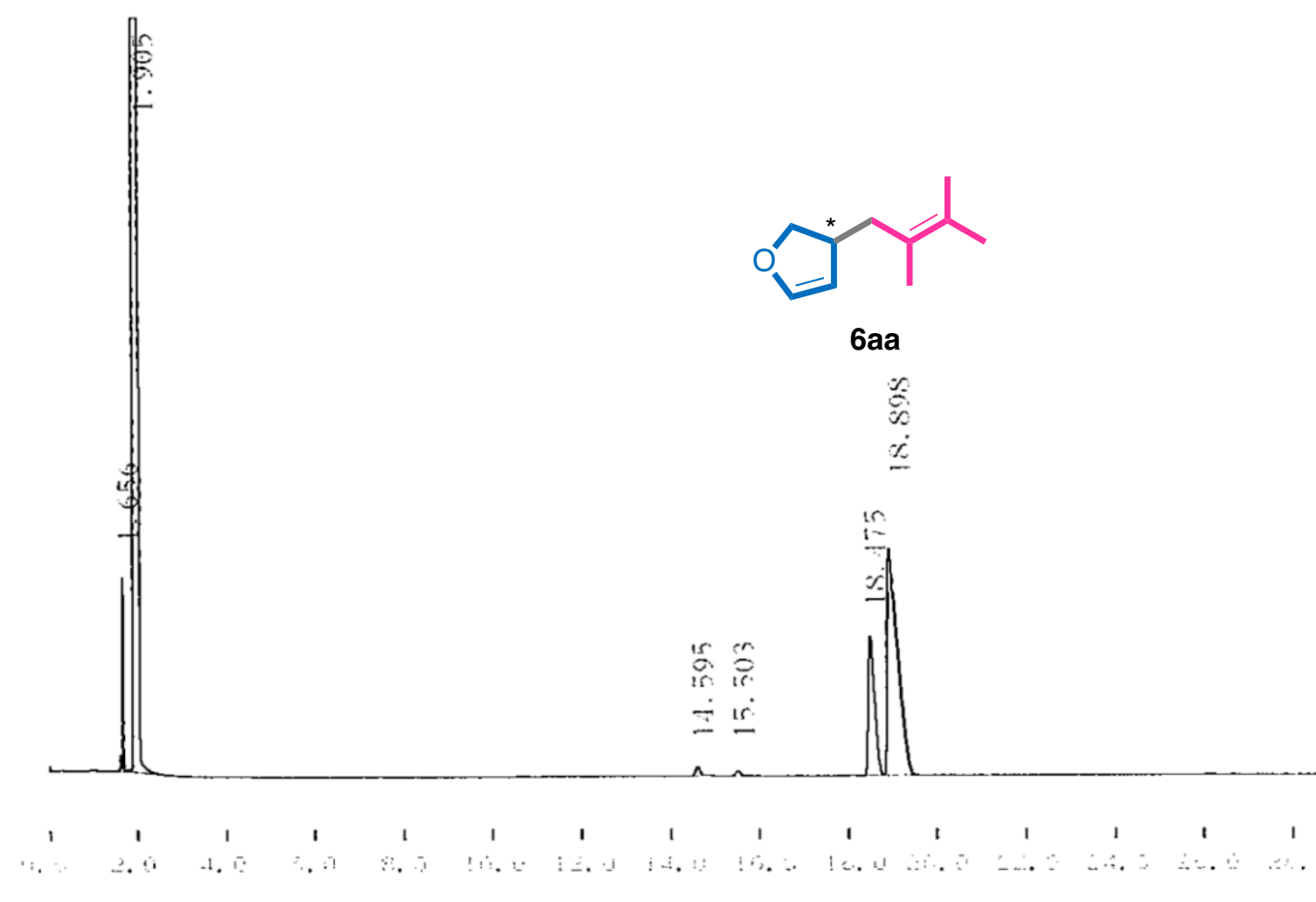

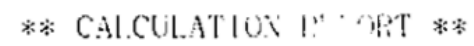

CH PKNO TIME AKEA HEIGIT HK IIAO CON

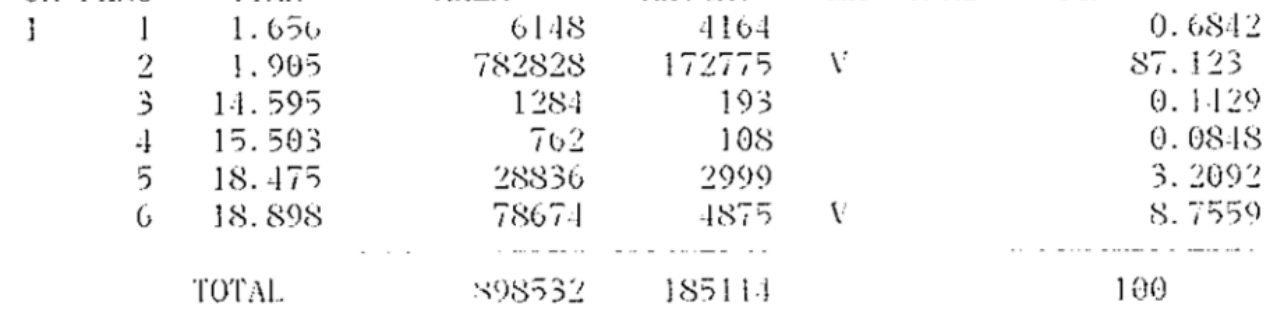

ent-6aa: $\mathrm{RT}=18.475,18.898 \mathrm{~min}$.

ee $=(78674-28836) /(78674+28836)=46 \%$ ee $(S)$

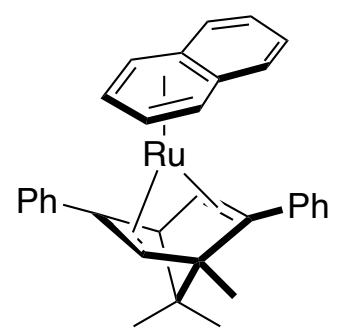

$2 a$

Figure S37-2. CSP-GC Chromatogram of ent-6aa produced by the catalysis using $2 \mathrm{a}(10 \mathrm{~mol} \%)$ at $0{ }^{\circ} \mathrm{C}$ in acetone. Conditions: Column: Rt- $\beta$ DEXsm (0.25 mmf x $30 \mathrm{~m})$, oven temp: $90{ }^{\circ} \mathrm{C}$, linear velosity: $40 \mathrm{~cm}$ $\mathrm{S}^{-1}$. 

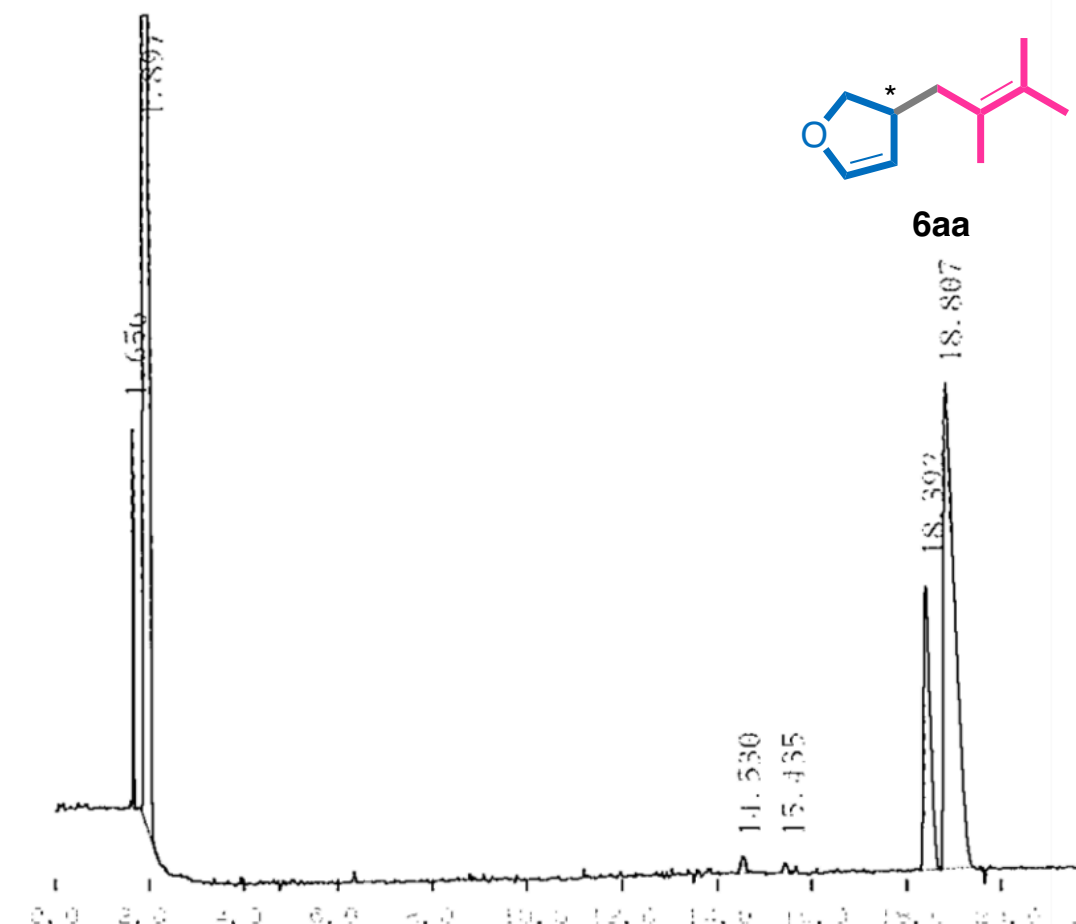

6aa

兽

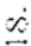

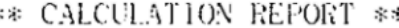

\begin{tabular}{|c|c|c|c|c|c|}
\hline PliNO & TIM: & MHI & HE:IGITT & Sll IINO & $\cos { }^{\circ}$ \\
\hline 2 & 1.656 & 4975 & 3801 & & 0.5017 \\
\hline 3 & 1.897 & 879762 & 190.100 & & 88.7212 \\
\hline 5 & 14.53 & 1202 & 175 & & 0.1212 \\
\hline 6 & 15.435 & 505 & 103 & & 0.057 \\
\hline 7 & 18. 392 & 26768 & 2855 & & 2.6995 \\
\hline$\$$ & 18.807 & 78331 & -4590 & $i$ & $\bar{i} . \$ 944$ \\
\hline & TAl. & 991602 & 202230 & & 100 \\
\hline
\end{tabular}

ent-6aa: $\mathrm{RT}=18.392,18.807 \mathrm{~min}$.

ee $=(78331-26768) /(78331+26768)=49 \%$ ee $(S)$

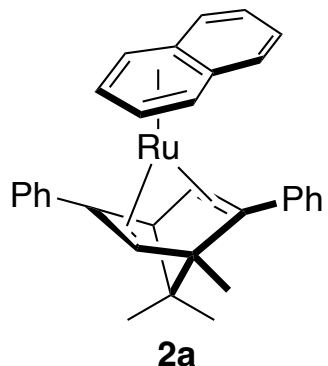

Figure S37-3. CSP-GC Chromatogram of ent-6aa produced by the catalysis using $2 \mathbf{2 a}(5 \mathrm{~mol} \%)$ at $30^{\circ} \mathrm{C}$ in acetone. Conditions: Column: Rt- $\beta$ DEXsm (0.25 mmf x $30 \mathrm{~m})$, oven temp: $90^{\circ} \mathrm{C}$, linear velosity: $40 \mathrm{~cm}$ $\mathrm{s}^{-1}$. 

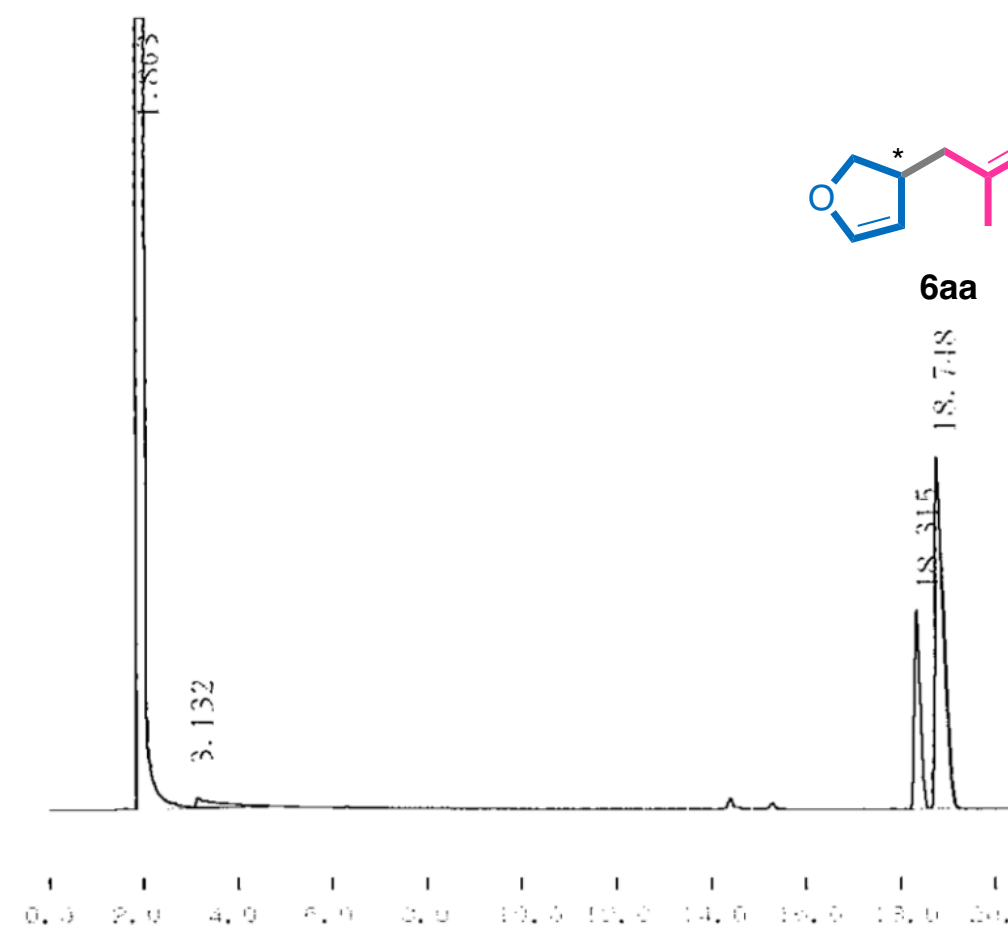

* CALCLLATION REPOH'T *

\begin{tabular}{|c|c|c|c|c|c|c|}
\hline CH PKNO & TIME: & ARE & Hil:!GHT & Ilk & ILNO & CONC \\
\hline 1 & 1.803 & $163086 ?$ & 30482.1 & S & & 90.16 .19 \\
\hline .4 & 3.132 & 2981 & 94 & $\tau$ & & 0.1759 \\
\hline 9 & 18.315 & 17312 & 1988 & V & & 1.0208 \\
\hline 10 & 18.7 .18 & 14743 & 3529 & V & & 2.6383 \\
\hline & TOTAL & 1695901 & 310.135 & & & 100 \\
\hline
\end{tabular}

ent-6aa: $\mathrm{RT}=18.315,18.748 \mathrm{~min}$.

ee $=(44743-17312) /(44743+17312)=44 \%$ ee $(S)$

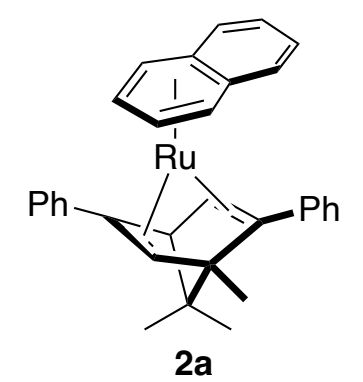

Figure 537-4. CSP-GC Chromatogram of ent-6aa produced by the catalysis using $\mathbf{2 a}(0.5 \mathrm{~mol} \%)$ at r.t. in acetone. Conditions: Column: Rt- $\beta$ DEXsm $(0.25 \mathrm{mmf} \times 30 \mathrm{~m})$, oven temp: $90^{\circ} \mathrm{C}$, linear velosity: $40 \mathrm{~cm} \mathrm{~s}^{-1}$. 
$\mathrm{mV}$

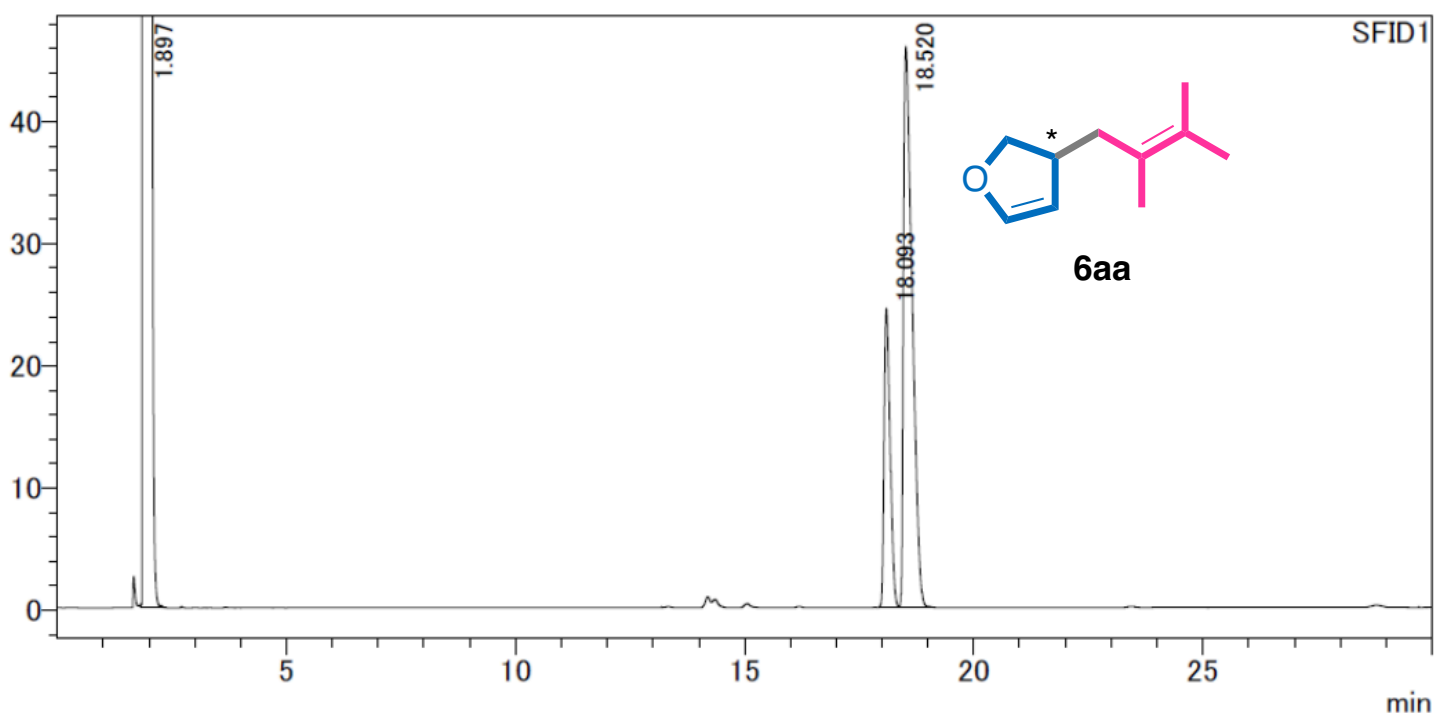

\begin{tabular}{|r|r|r|r|r|}
\hline Peak \# & \multicolumn{2}{l|}{$\begin{array}{l}\text { Retention } \\
\text { Time }\end{array}$} & \multicolumn{1}{l|}{ Area } & Hight \\
\hline 1 & 18.093 & 226124 & 24443 & 0.996 \\
\hline 2 & 18.52 & 611400 & 45905 & 2.692 \\
\hline
\end{tabular}

ent-6aa: $\mathrm{RT}=18.093,18.520 \mathrm{~min}$.

$\mathrm{ee}=(611400-22614) /(611400+226124)=46 \%$ ee $(S)$

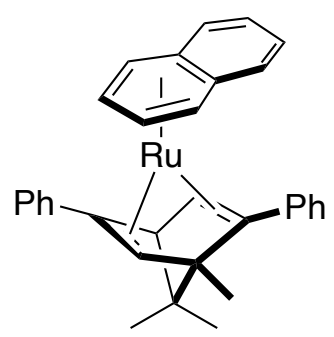

$2 a$

Figure S37-5. CSP-GC Chromatogram of ent- 6 aa produced by the catalysis using $2 \mathbf{a}(5 \mathrm{~mol} \%)$ at $30{ }^{\circ} \mathrm{C}$ in dmf. Conditions: Column: Rt- $\beta$ DEXsm (0.25 mmf x $30 \mathrm{~m})$, oven temp: $90^{\circ} \mathrm{C}$, linear velosity: $40 \mathrm{~cm} \mathrm{~s}^{-1}$. 
$\mathrm{uV}$

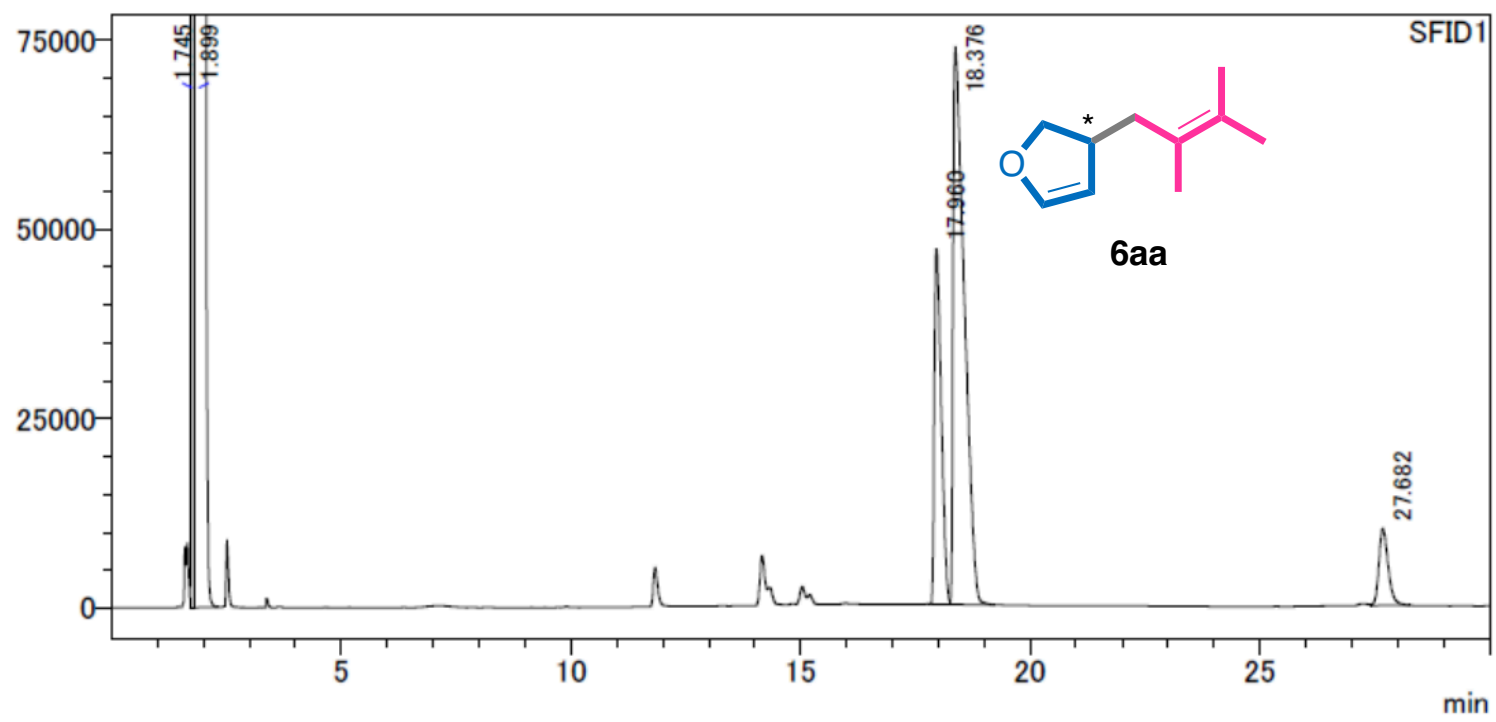

\begin{tabular}{|r|r|r|r|r|}
\hline Peak \# & \multicolumn{2}{l|}{$\begin{array}{l}\text { Retention } \\
\text { Time }\end{array}$} & \multicolumn{1}{l|}{ Area } & Hight \\
\hline 1 & 17.96 & 495855 & 47014 & 1.121 \\
\hline 2 & 18.376 & 1248769 & 73678 & 2.823 \\
\hline
\end{tabular}

ent-6aa: $\mathrm{RT}=17.960,18.376 \mathrm{~min}$.

$\mathrm{ee}=(1248769-4958755) /(1248769+495855)=43 \%$ ee $(S)$

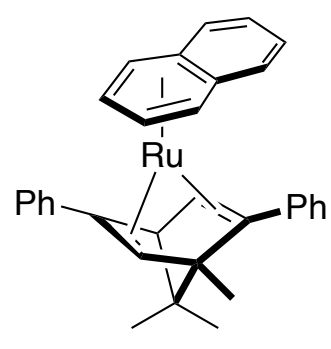

$2 a$

Figure S37-6. CSP-GC Chromatogram of ent-6aa produced by the catalysis using $2 \mathrm{a}(5 \mathrm{~mol} \%)$ at $30^{\circ} \mathrm{C}$ in thf. Conditions: Column: Rt- $\beta$ DEXsm $(0.25 \mathrm{mmf} \times 30 \mathrm{~m})$, oven temp: $90^{\circ} \mathrm{C}$, linear velosity: $40 \mathrm{~cm} \mathrm{~s}^{-1}$. 
$\mathrm{m}$ V

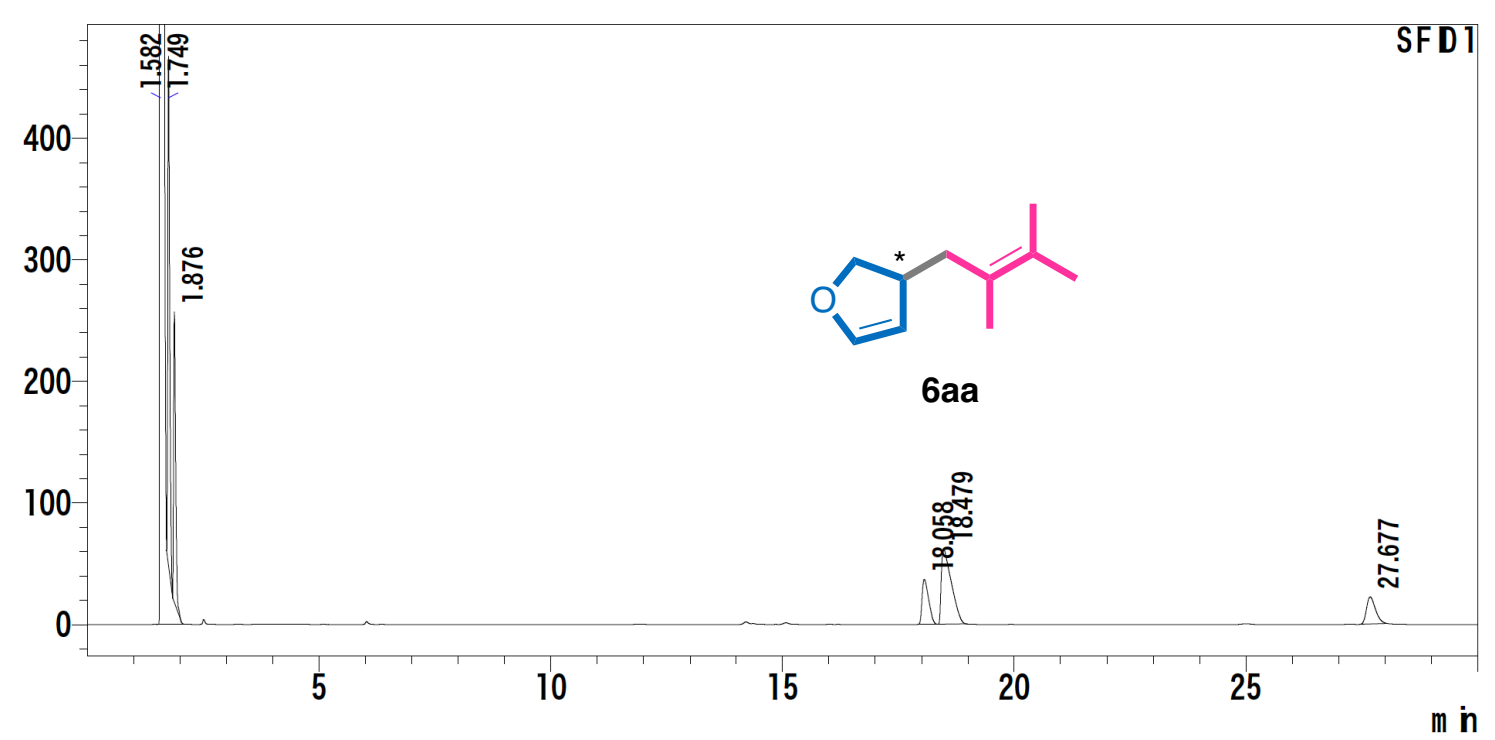

\begin{tabular}{|r|r|r|r|r|}
\hline Peak \# & \multicolumn{1}{l|}{$\begin{array}{l}\text { Retention } \\
\text { Time }\end{array}$} & \multicolumn{1}{l|}{ Area } & Hight \\
\hline 1 & 18.058 & 365082 & 37012 & 1.045 \\
\hline 2 & 18.479 & 930048 & 61742 & 2.663 \\
\hline
\end{tabular}

ent-6aa: $\mathrm{RT}=18.058,18.479 \mathrm{~min}$

ee $=(930048-365082) /(930048+365082)=44 \%$ ee $(S)$

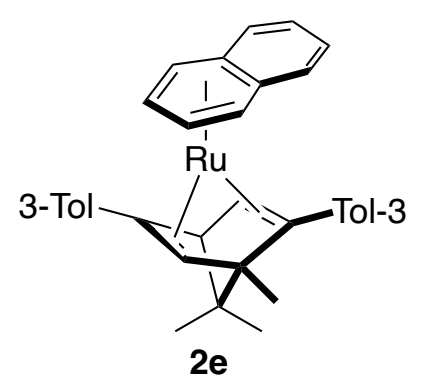

Figure S37-7. CSP-GC Chromatogram of ent-6aa produced by the catalysis using 2e. Conditions:

Column: Rt- $\beta$ DEXsm $(0.25 \mathrm{mmf} \times 30 \mathrm{~m})$, oven temp: $90^{\circ} \mathrm{C}$, linear velosity: $40 \mathrm{~cm} \mathrm{~s}^{-1}$. 
$\mathrm{mV}$

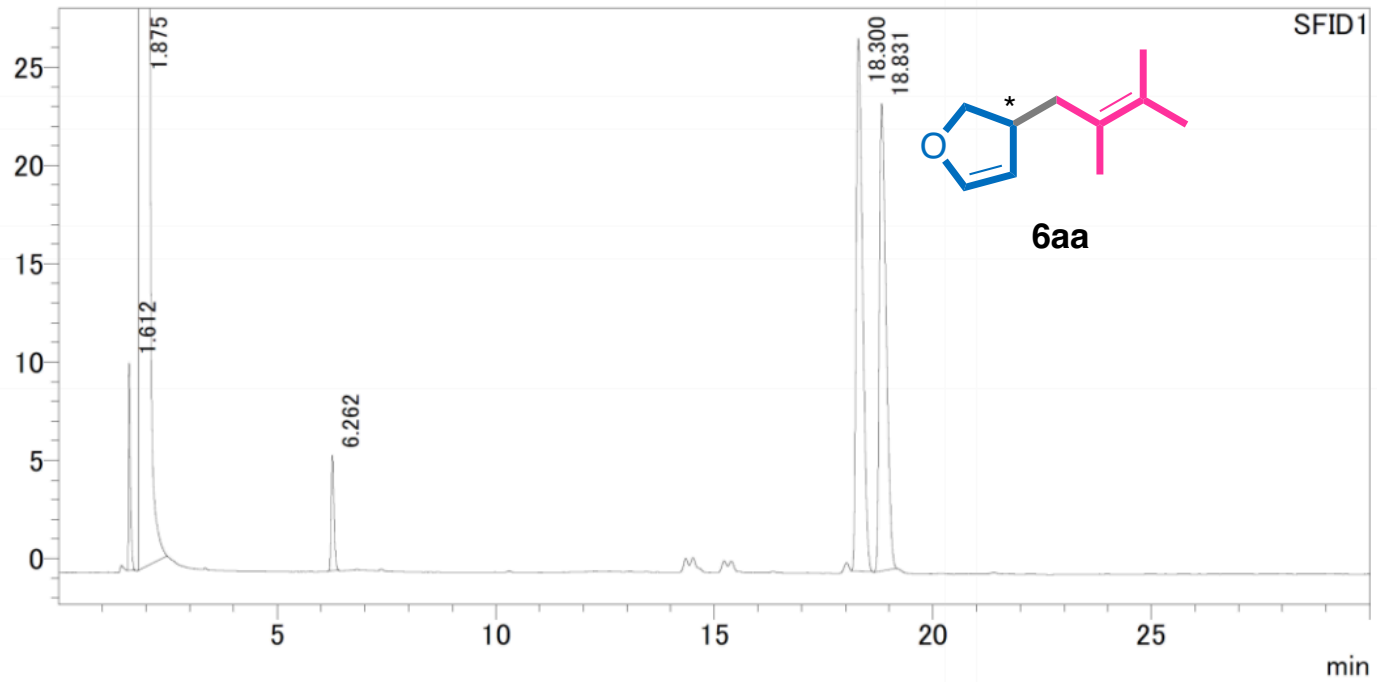

\begin{tabular}{|r|r|r|r|r|}
\hline Peak \# & \multicolumn{2}{l|}{$\begin{array}{l}\text { Retention } \\
\text { Time }\end{array}$} & \multicolumn{1}{l|}{ Area } & Hight \\
\hline 1 & 18.3 & 275986 & 27080 & 0.881 \\
\hline 2 & 18.831 & 260768 & 23747 & 0.832 \\
\hline
\end{tabular}

ent-6aa: $\mathrm{RT}=18.300,18.831 \mathrm{~min}$.

ee $=(275986-260768) /(275986+260768)=3 \%$ ee $(R)$

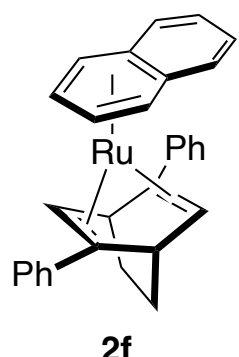

Figure S37-8. CSP-GC Chromatogram of ent-6aa produced by the catalysis using 2f. Conditions: Column: Rt- $\beta$ DEXsm (0.25 mmf x $30 \mathrm{~m})$, oven temp: $90^{\circ} \mathrm{C}$, linear velosity: $40 \mathrm{~cm} \mathrm{~s}^{-1}$. 


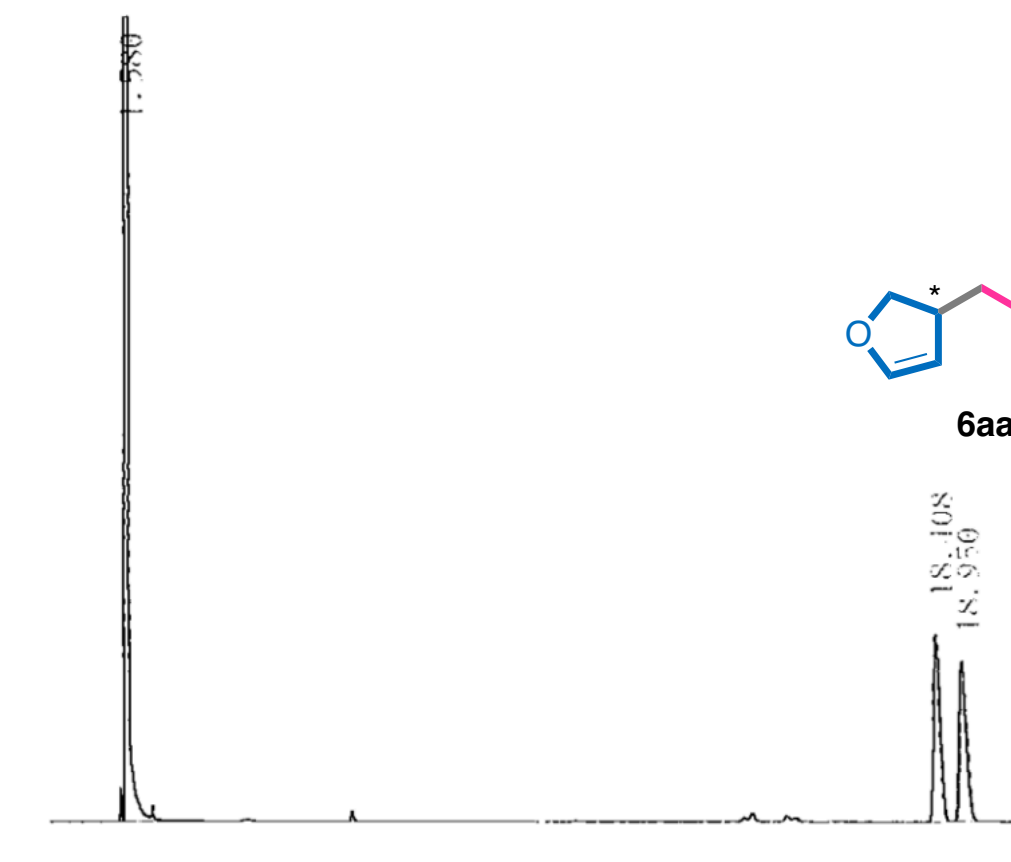

* CAICULATON REPOHT * *

\begin{tabular}{|c|c|c|c|c|c|c|}
\hline $\mathrm{CH}$ lWNo & TISIE & AlEA & $\mathrm{HE}: \mathrm{IGH}$ ! & IIK & 11) & COAC \\
\hline 2 & 1. $5 \$$ & 3171001 & 1338143 & $\mathrm{~s}$ & & 98.9072 \\
\hline 13 & 18. 408 & $171.1 !$ & 1855 & 1 & & 0.33 .19 \\
\hline $1+4$ & 18.95 & 15955 & 1578 & & & 0.1979 \\
\hline
\end{tabular}

TOTAL.

$3204608 \quad 13415 \%$

100

ent-6aa: $\mathrm{RT}=18.408,18.950 \mathrm{~min}$.

ee $=(17141-15955) /(17141+15955)=4 \%$ ee $(R)$

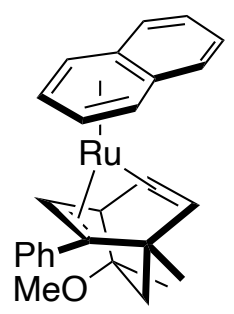

$2 \mathrm{~g}$

Figure S37-9. CSP-GC Chromatogram of ent-6aa produced by the catalysis using $\mathbf{2 g}$. Conditions: Column: Rt- $\beta$ DEXsm $(0.25 \mathrm{mmf} \times 30 \mathrm{~m})$, oven temp: $90^{\circ} \mathrm{C}$, linear velosity: $40 \mathrm{~cm} \mathrm{~s}^{-1}$. 


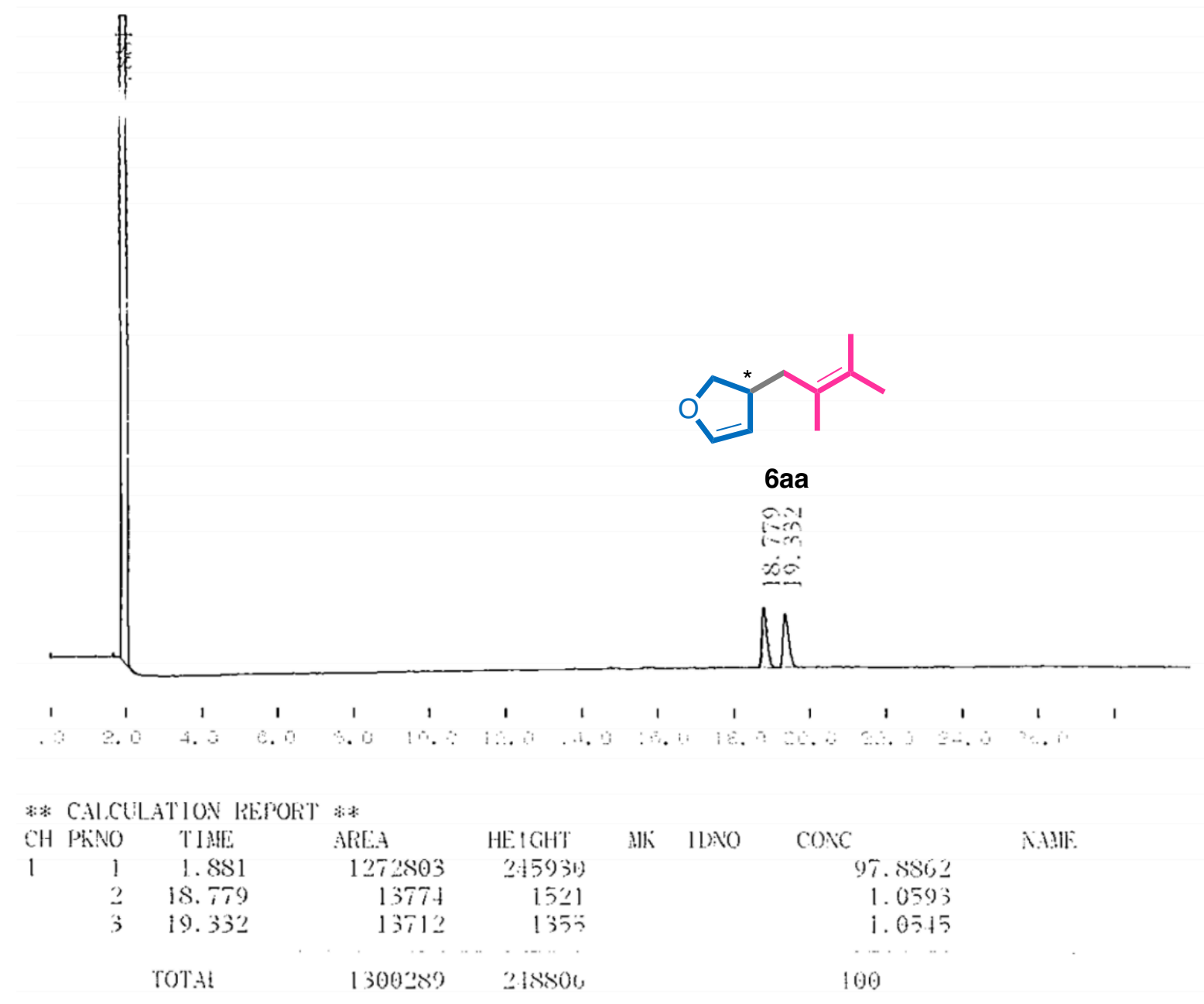

ent-6aa: $\mathrm{RT}=18.779,19.332 \mathrm{~min}$.

$\mathrm{ee}=(13774-13712) /(13774+13712)=0 \%$ ee

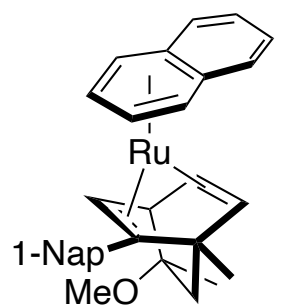

2h

Figure S37-10. CSP-GC Chromatogram of ent-6aa produced by the catalysis using $\mathbf{2 h}$. Conditions: Column: Rt- $\beta$ DEXsm $(0.25 \mathrm{mmf}$ x $30 \mathrm{~m})$, oven temp: $90^{\circ} \mathrm{C}$, linear velosity: $40 \mathrm{~cm} \mathrm{~s}^{-1}$. 


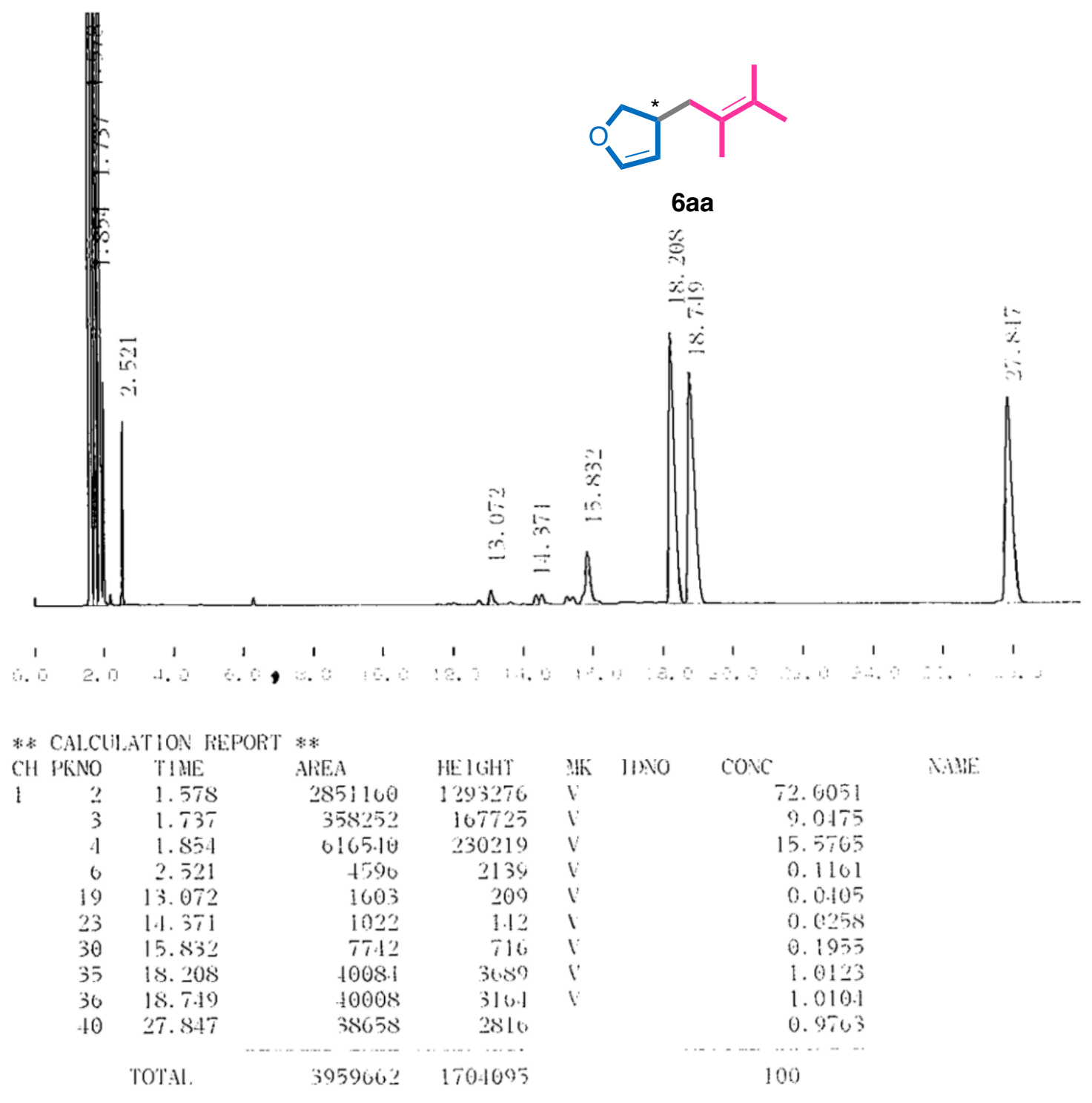

ent-6aa: $\mathrm{RT}=18.208,18.749 \mathrm{~min}$.

ee $=(40084-40008) /(40084+40008)=0 \%$ ee

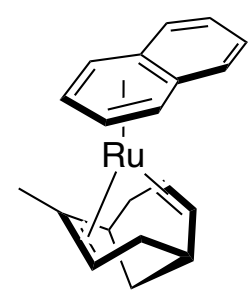

2i

Figure S37-11. CSP-GC Chromatogram of ent-6aa produced by the catalysis using 2i. Conditions: Column: Rt- $\beta$ DEXsm (0.25 mmf x $30 \mathrm{~m})$, oven temp: $90^{\circ} \mathrm{C}$, linear velosity: $40 \mathrm{~cm} \mathrm{~s}^{-1}$. 

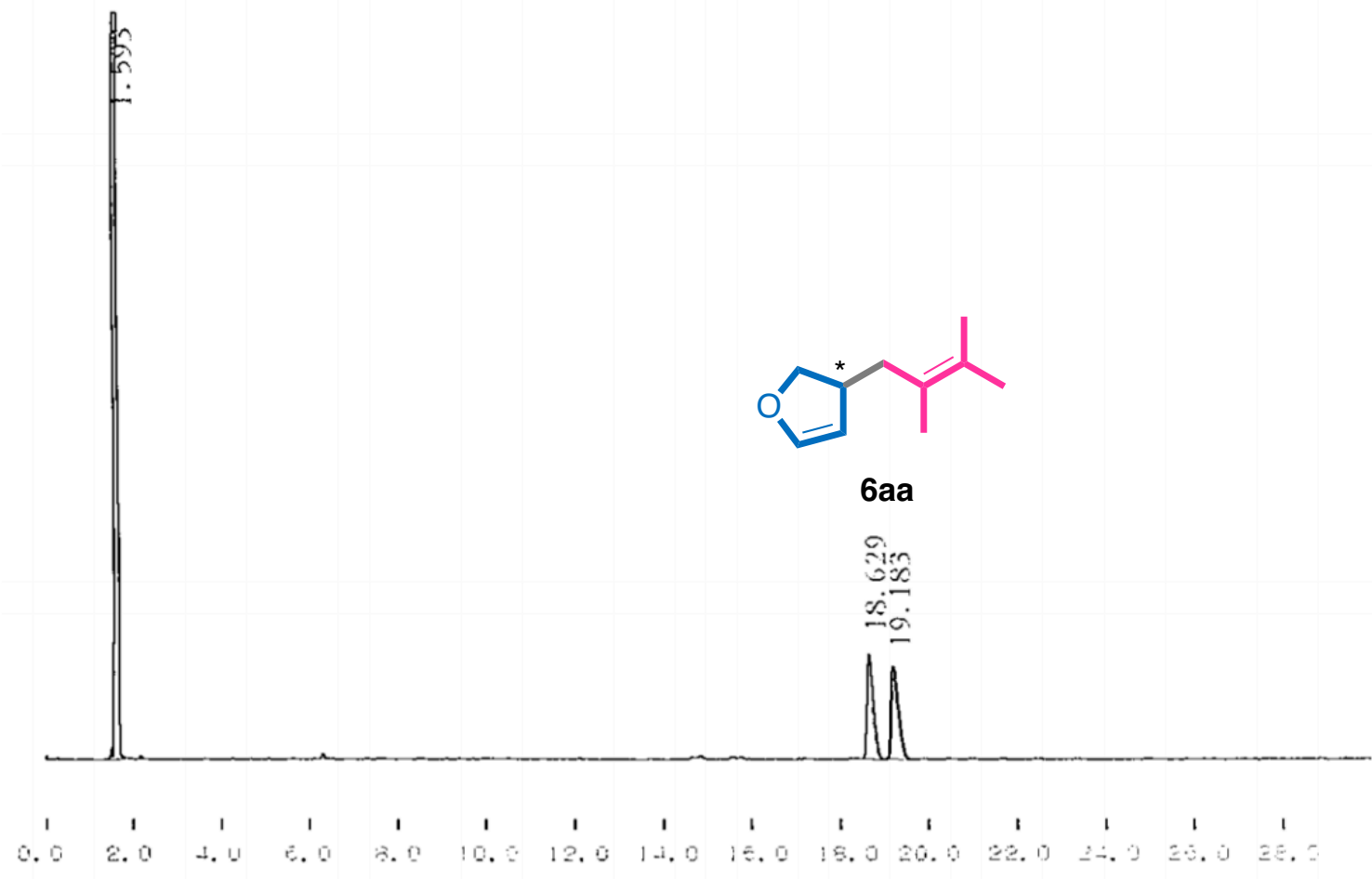

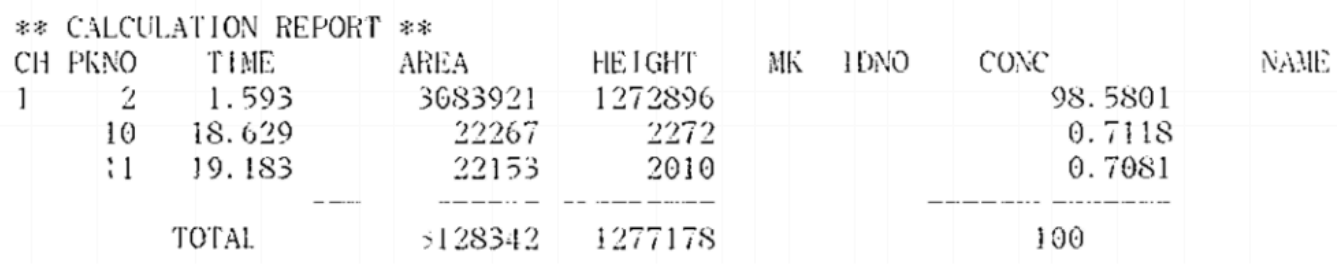

ent-6aa: $\mathrm{RT}=18.629,19.183 \mathrm{~min}$.

$\mathrm{ee}=(22267-22153) /(22267+22153)=0 \%$ ee

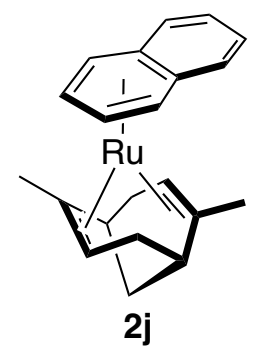

Figure S37-12. CSP-GC Chromatogram of ent-6aa produced by the catalysis using $\mathbf{2} \mathbf{j}$. Conditions: Column: Rt- $\beta$ DEXsm $(0.25 \mathrm{mmf} \times 30 \mathrm{~m})$, oven temp: $90^{\circ} \mathrm{C}$, linear velosity: $40 \mathrm{~cm} \mathrm{~s}^{-1}$. 

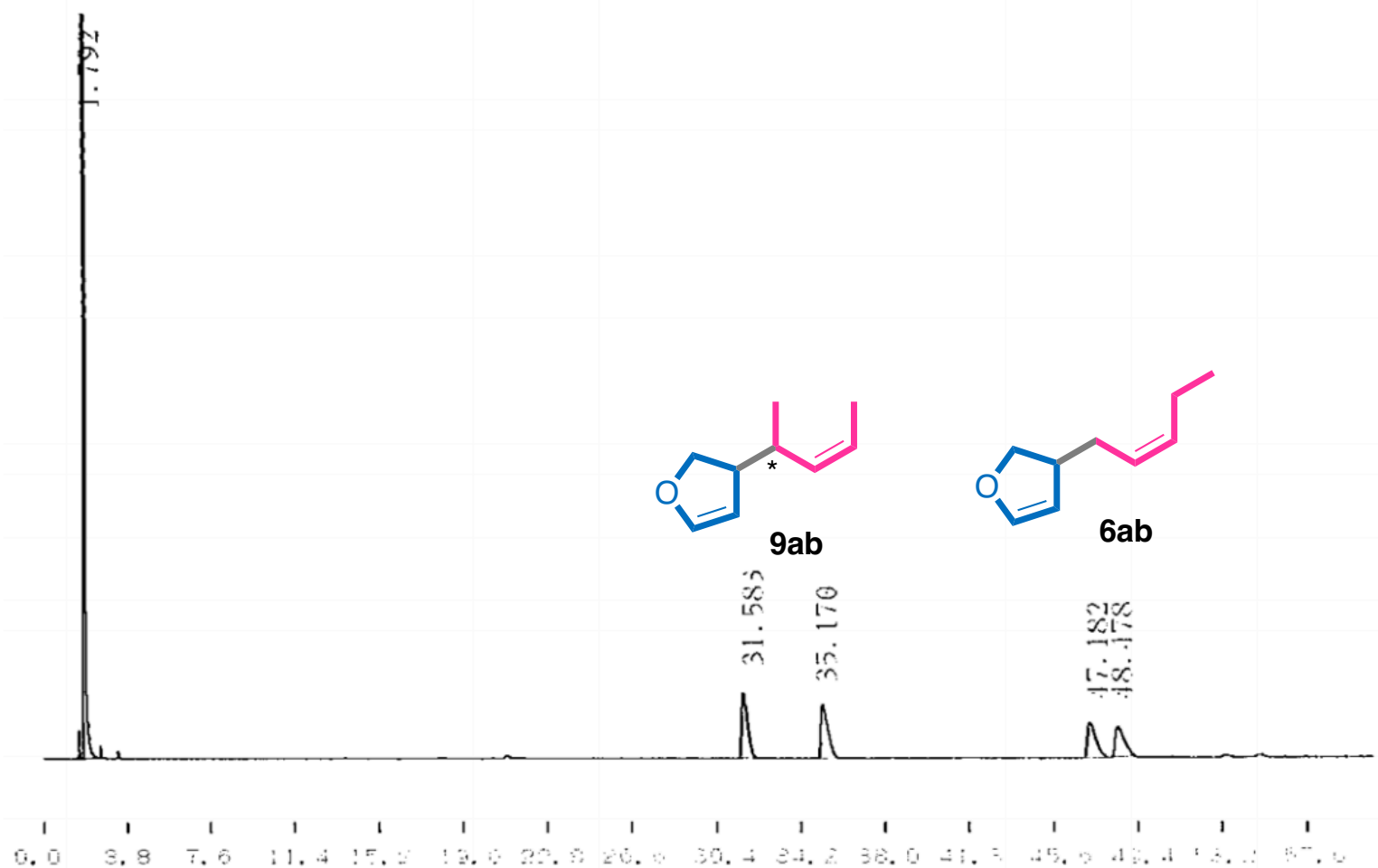

** CALCULATION REPORT **:

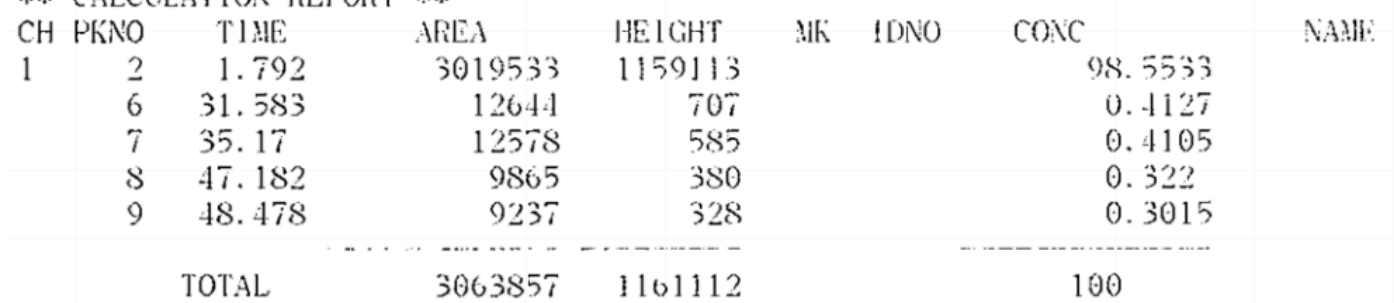

rac-9ab: $\mathrm{RT}=31.583,35.170 \mathrm{~min}$.

rac-7ab: 47.182, $48.478 \mathrm{~min}$.

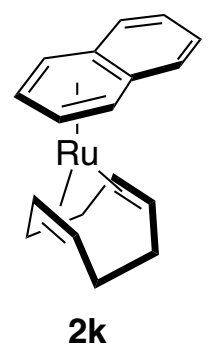

Figure S38-1. CSP-GC Chromatogram of rac-6ab and rac-9ab produced by the catalysis using $\mathbf{2 k}$. Conditions: Column: Rt- $\beta$ DEXsm $(0.25 \mathrm{mmf} \times 30 \mathrm{~m})$, oven temp: $60{ }^{\circ} \mathrm{C}$, linear velosity: $40 \mathrm{~cm} \mathrm{~s}^{-1}$. 


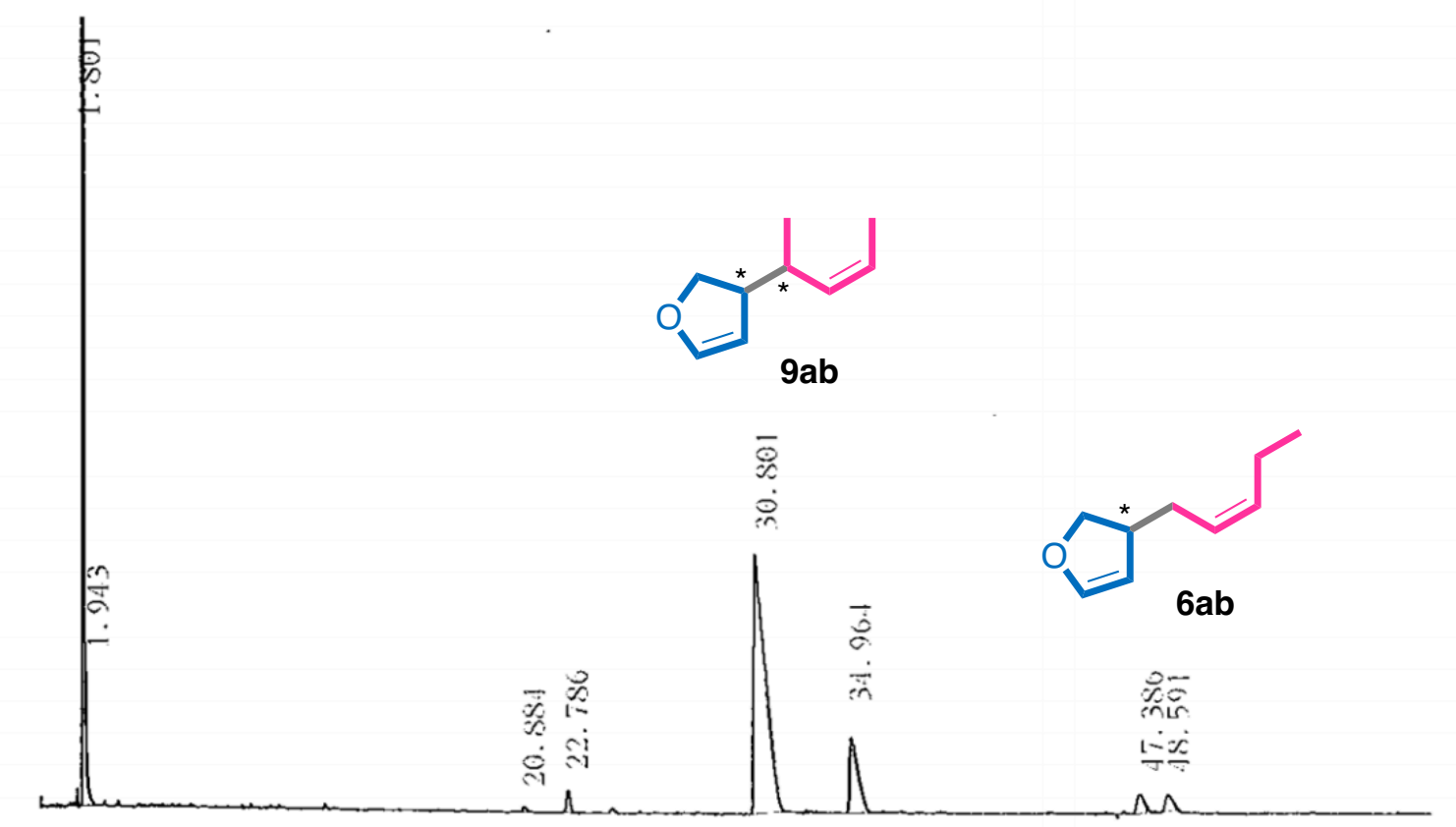

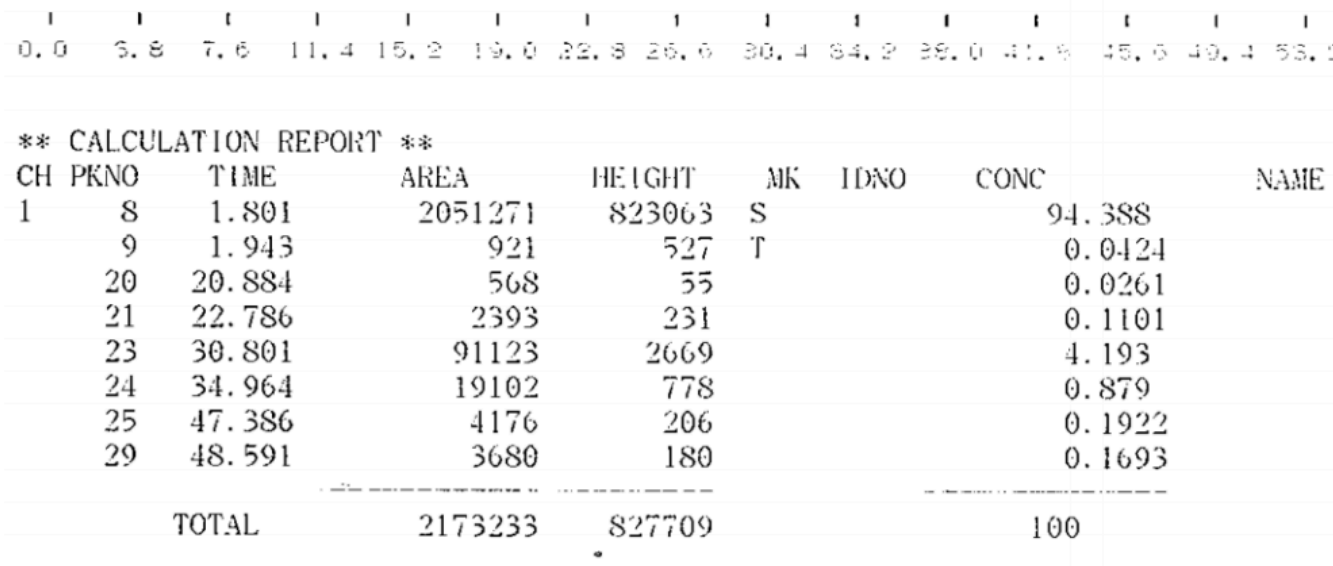

ent-9ab: $\mathrm{RT}=30.801,34.964 \mathrm{~min}$.

ee $=(91123-19102) /(91123+19102)=65 \%$ ee

ent-6ab: $\mathrm{RT}=47.386,48.591 \mathrm{~min}$.

ee $=(4176-3680) /(4176+3680)=6 \%$ ee

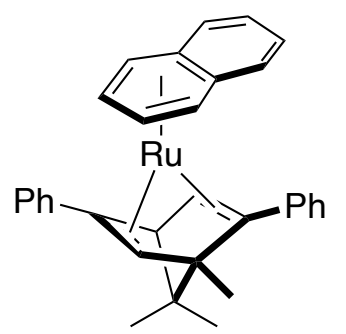

2a

Figure S39-1. CSP-GC Chromatogram of ent-6ab and ent-9ab produced by the catalysis using $\mathbf{2 a}$ at r.t.. Conditions: Column: Rt- $\beta$ DEXsm (0.25 mmf x $30 \mathrm{~m})$, oven temp: $60^{\circ} \mathrm{C}$, linear velosity: $40 \mathrm{~cm} \mathrm{~s}^{-1}$ 

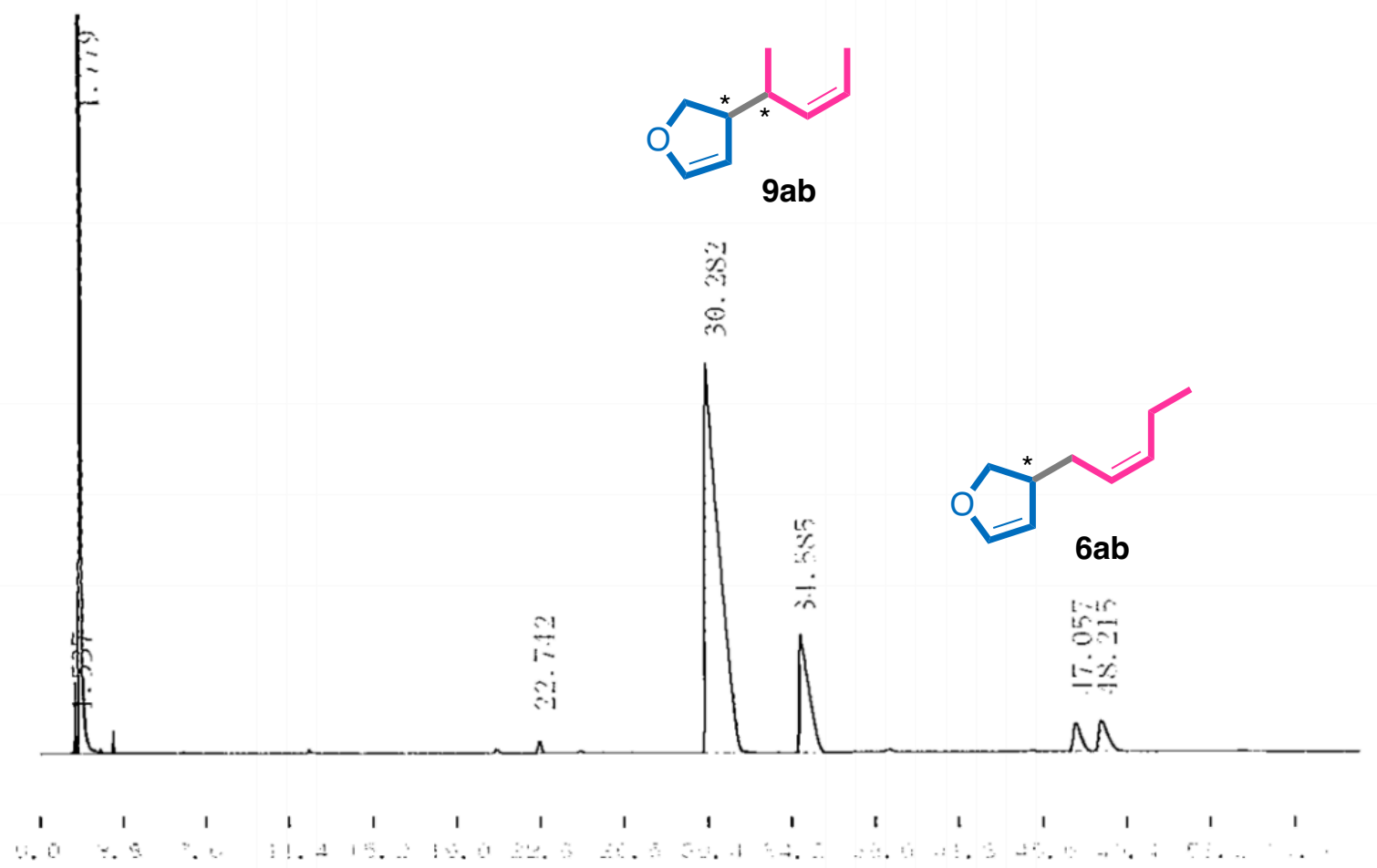

* CALCTLATION RLPOPRT **

\begin{tabular}{|c|c|c|c|c|c|c|}
\hline CH PKNO & TIME & ARIA & HEIGHT & !IK & IDNO & CON: \\
\hline 1 & 1.537 & 1315 & 99 & & & 0.0178 \\
\hline 2 & 1.779 & 7296249 & 282393 & SV & & 9.7612 \\
\hline 22 & 22.712 & $141 \bar{i}$ & 135 & & & $0.018 s$ \\
\hline 24 & 30.282 & 187332 & 4212 & & & $2.48+4$ \\
\hline 25 & 34.585 & $37+9 \mathrm{i}$ & 1269 & & & 0.4972 \\
\hline 30 & 47.057 & 7321 & 312 & & & 0.0971 \\
\hline 31 & 48.215 & 9314 & 331 & l & & 0.1235 \\
\hline & TOTAL. & 75.10168 & $2 \operatorname{sis} 650$ & & & 100 \\
\hline
\end{tabular}

ent-9ab: $\mathrm{RT}=30.282,34.585 \mathrm{~min}$.

ee $=(187332-37491) /(187332+37491)=67 \%$ ee

ent-6ab: $\mathrm{RT}=47.057,48.215 \mathrm{~min}$.

ee $=(9314-7321) /(9314+7321)=12 \%$ ee

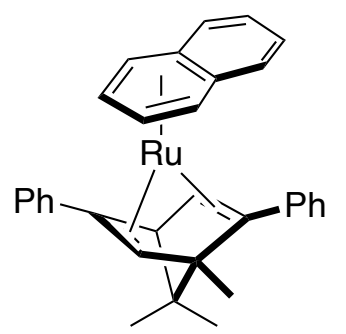

$2 a$

Figure S39-2. CSP-GC Chromatogram of ent-6ab and ent-9ab produced by the catalysis using $2 \mathbf{a}$ at $0{ }^{\circ} \mathrm{C}$ Conditions: Column: Rt- $\beta$ DEXsm $(0.25 \mathrm{mmf} \times 30 \mathrm{~m})$, oven temp: $60{ }^{\circ} \mathrm{C}$, linear velosity: $40 \mathrm{~cm} \mathrm{~s}^{-1}$ 

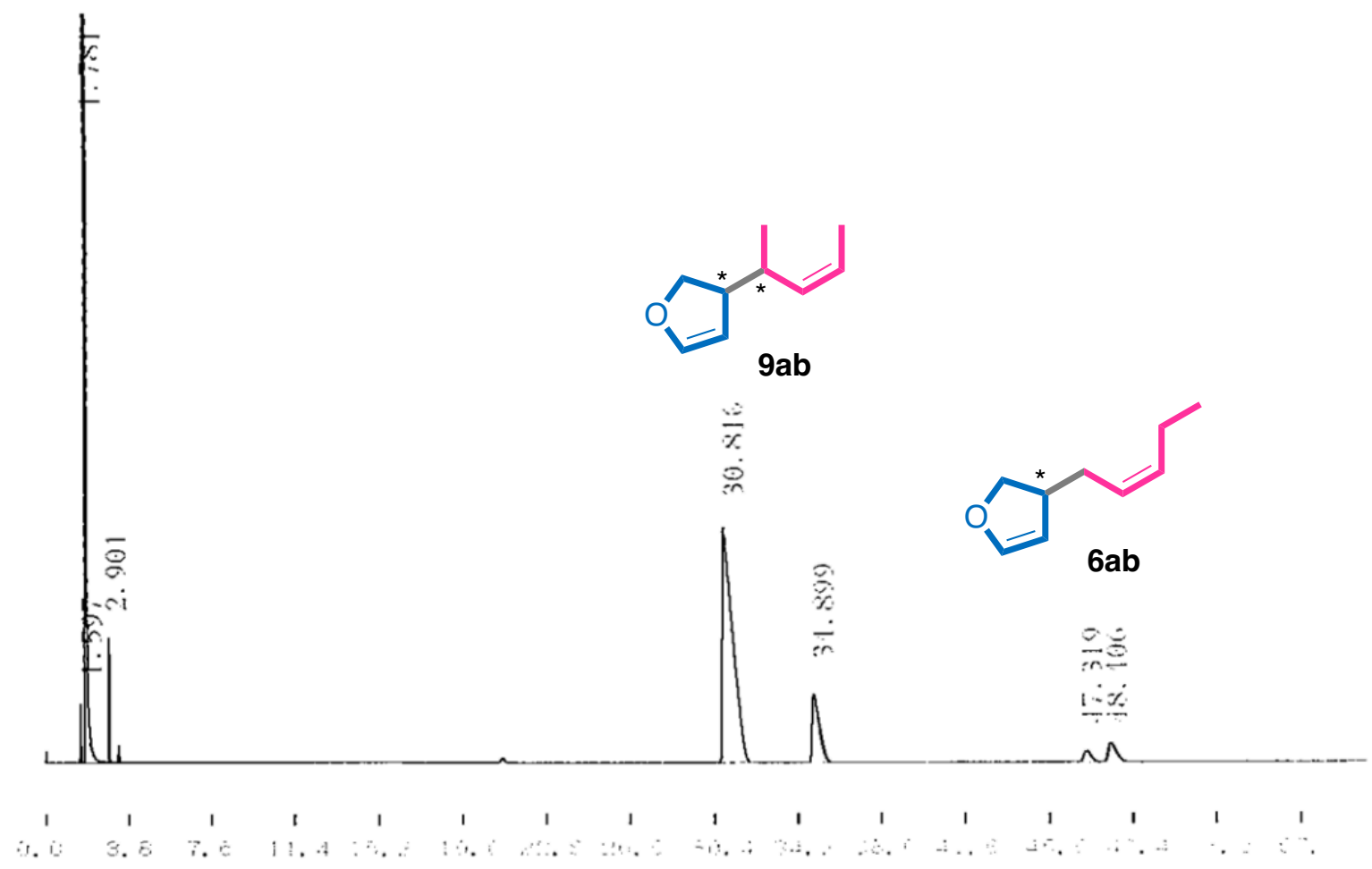

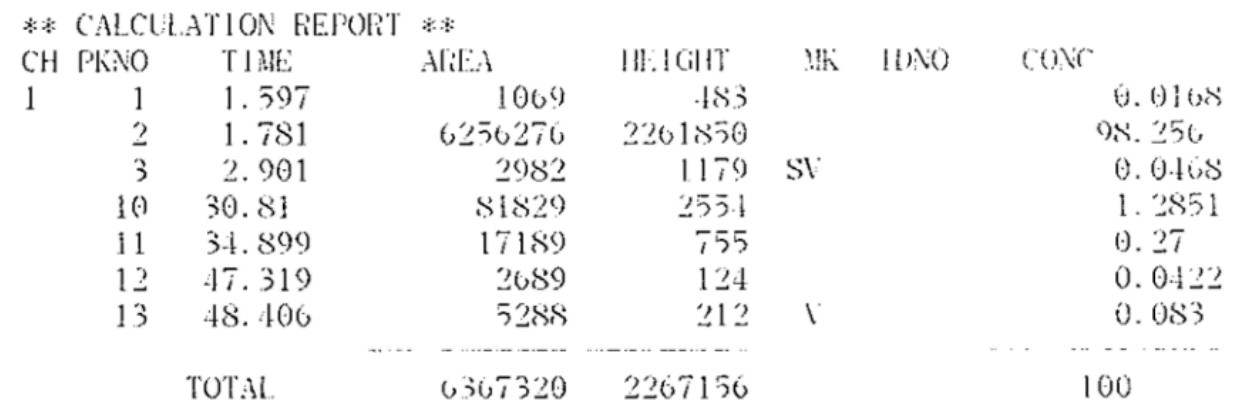

ent-9ab: RT $=30.810,34.899 \mathrm{~min}$.

ee $=(81829-17189) /(81829+17189)=65 \%$ ee ent-6ab: $\mathrm{RT}=47.319,48.406 \mathrm{~min}$.

ee $=(5288-2689) /(5288+2689)=33 \%$ ee

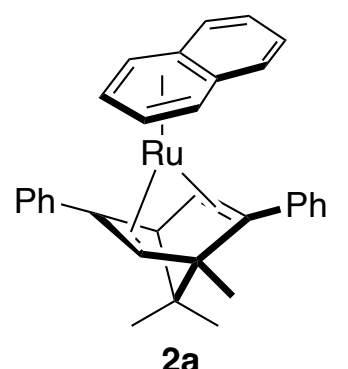

2a

Figure S39-3. CSP-GC Chromatogram of ent-6ab and ent-9ab produced by the catalysis using $\mathbf{2 a}$ at -40 ${ }^{\circ} \mathrm{C}$ Conditions: Column: Rt- $\beta$ DEXsm $(0.25 \mathrm{mmf} \times 30 \mathrm{~m})$, oven temp: $60{ }^{\circ} \mathrm{C}$, linear velosity: $40 \mathrm{~cm} \mathrm{~s}^{-1}$. 


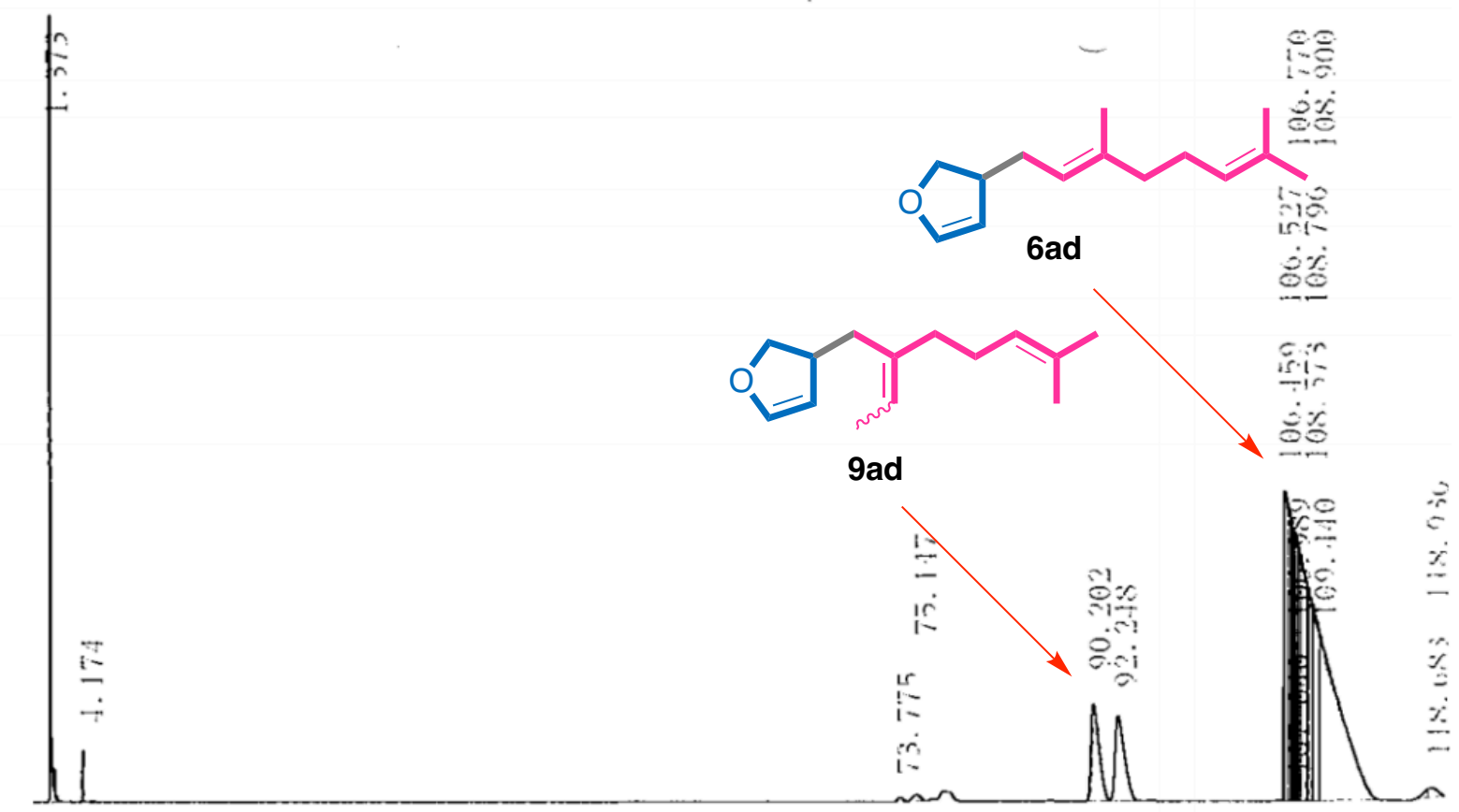

$\begin{array}{ccccc}1 & 1 & 1 & 1 \\ 0.1 & 7.7 & 1 & 4 & -3\end{array}$

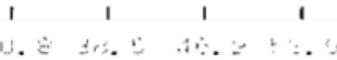

* CAICULATION REPORT **

\begin{tabular}{|c|c|c|c|c|c|}
\hline $\mathrm{CH}$ PhNO & TIME & AlREA & I IE [GIIT & HK $112 \mathrm{NO}$ & $\mathrm{CONC}$ \\
\hline 1 & 1.373 & 6189060 & 2490249 & & 89.1 .192 \\
\hline 3 & 4.174 & 1257 & 500 & & 0.0181 \\
\hline 8 & 73.775 & 1229 & 37 & & 0.0177 \\
\hline 9 & 75.147 & 3083 & 65 & Vi & 0.0441 \\
\hline 12 & 90.202 & .15960 & 979 & & 0.602 \\
\hline 13 & 92.218 & 46591 & 859 & $y^{\prime}$ & 0.6711 \\
\hline 14 & 106.459 & 24051 & 3112 & & 0.3404 \\
\hline 15 & $100.52 \pi$ & .15010 & 3078 & V & 0.657 \\
\hline 16 & 106.77 & 38002 & 2990 & $r$ & $0 . \bar{j}-\bar{i}-1$ \\
\hline 17 & 106.989 & 8506 & 2867 & $V$ & 0.1225 \\
\hline 18 & 107.04 & 22467 & 2819 & $l$ & 0.3236 \\
\hline 19 & 107.154 & $10.13-1$ & 2855 & l & 0. \\
\hline 20 & 107.267 & $\sec 3$ & TAS & 1 & 0.1160 \\
\hline 21 & 107.321 & 10701 & 2726 & $r$ & 0.155 \\
\hline 23 & 107.375 & 797S & 2701 & 1 & 0.11 .19 \\
\hline 33 & 107.44 & 28908 & 2701 & $i$ & 0.4101 \\
\hline 24 & 107.615 & 15147 & $25+3$ & V & 0.2182 \\
\hline 25 & 107.733 & 27019 & $253 \%$ & V & 0. 3892 \\
\hline 26 & 107.907 & 02040 & 2398 & $V$ & 0.8930 \\
\hline 27 & 108.361 & 12900 & 2167 & V & 0.1850 \\
\hline 28 & 108.456 & 16902 & 2135 & V & $0.2+35$ \\
\hline 29 & 108.573 & 26346 & 2006 & $\mathrm{~V}$ & 0. 3795 \\
\hline 30 & 108.796 & $986 ?$ & 1984 & 1 & 0.112 \\
\hline 31 & 108.9 & 59612 & $197 \bar{i}$ & $i$ & 0.8591 \\
\hline 32 & 109.44 & 207503 & 1676 & 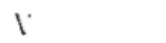 & "2. \\
\hline 33 & 118.683 & $26+3$ & 56 & & $0.03 \$ 1$ \\
\hline \multirow[t]{2}{*}{$3-1$} & 1 is. 936 & 4360 & 91 & 1 & 0.0629 \\
\hline & TOTAL. & 6942363 & 25.10913 & & 90.9999 \\
\hline
\end{tabular}

rac-9ad: $\mathrm{RT}=90.202,92.248 \mathrm{~min}$. 


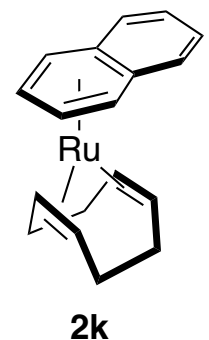

Figure S40-1. CSP-GC Chromatogram of rac-6ad and rac-9ad produced by the catalysis using $\mathbf{2 k}$ at r.t. Conditions: Column: Rt- $\beta$ DEXsm (0.25 mmf x $30 \mathrm{~m}$ ), oven temp: $100^{\circ} \mathrm{C}$, linear velosity: $45 \mathrm{~cm} \mathrm{~s}^{-1}$. Rac-6ad could not be separated under these conditions. 

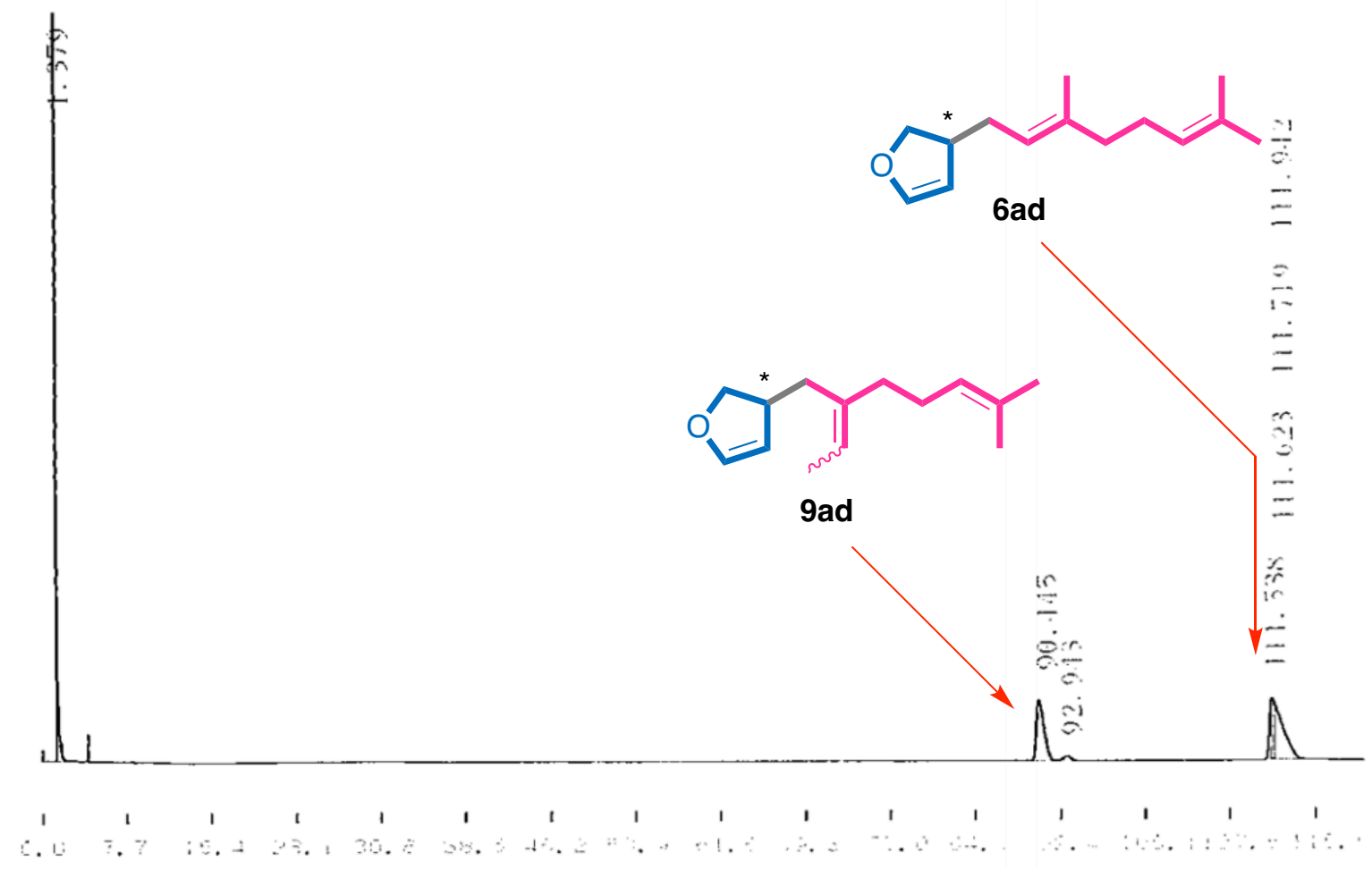

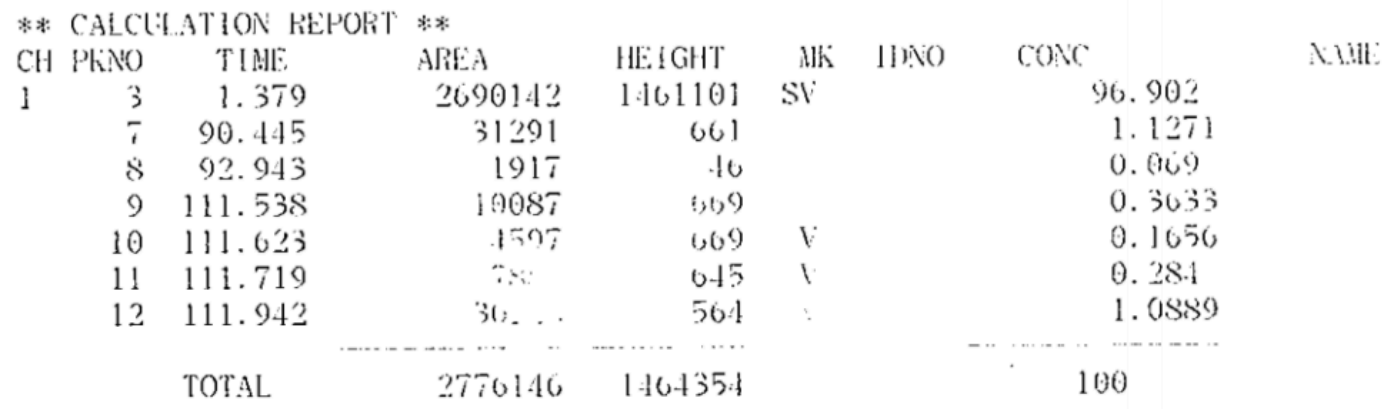

ent-9ad: $\mathrm{RT}=90.445,92.943 \mathrm{~min}$.

ee $=(31291-1917) /(31291+1917)=88 \%$ ee

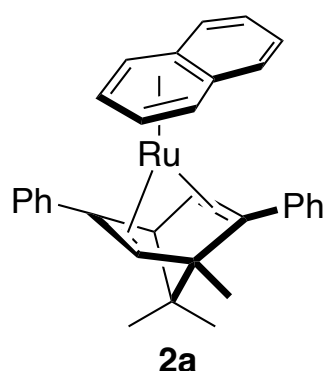

Figure S41-1. CSP-GC Chromatogram of ent-6ad and ent-9ad produced by the catalysis using $2 \mathbf{a}$ at r.t. Conditions: Column: Rt- $\beta$ DEXsm (0.25 mmf x $30 \mathrm{~m}$ ), oven temp: $100{ }^{\circ} \mathrm{C}$, linear velosity: $45 \mathrm{~cm} \mathrm{~s}^{-1}$. ent-6ad could not be separated under these conditions. 

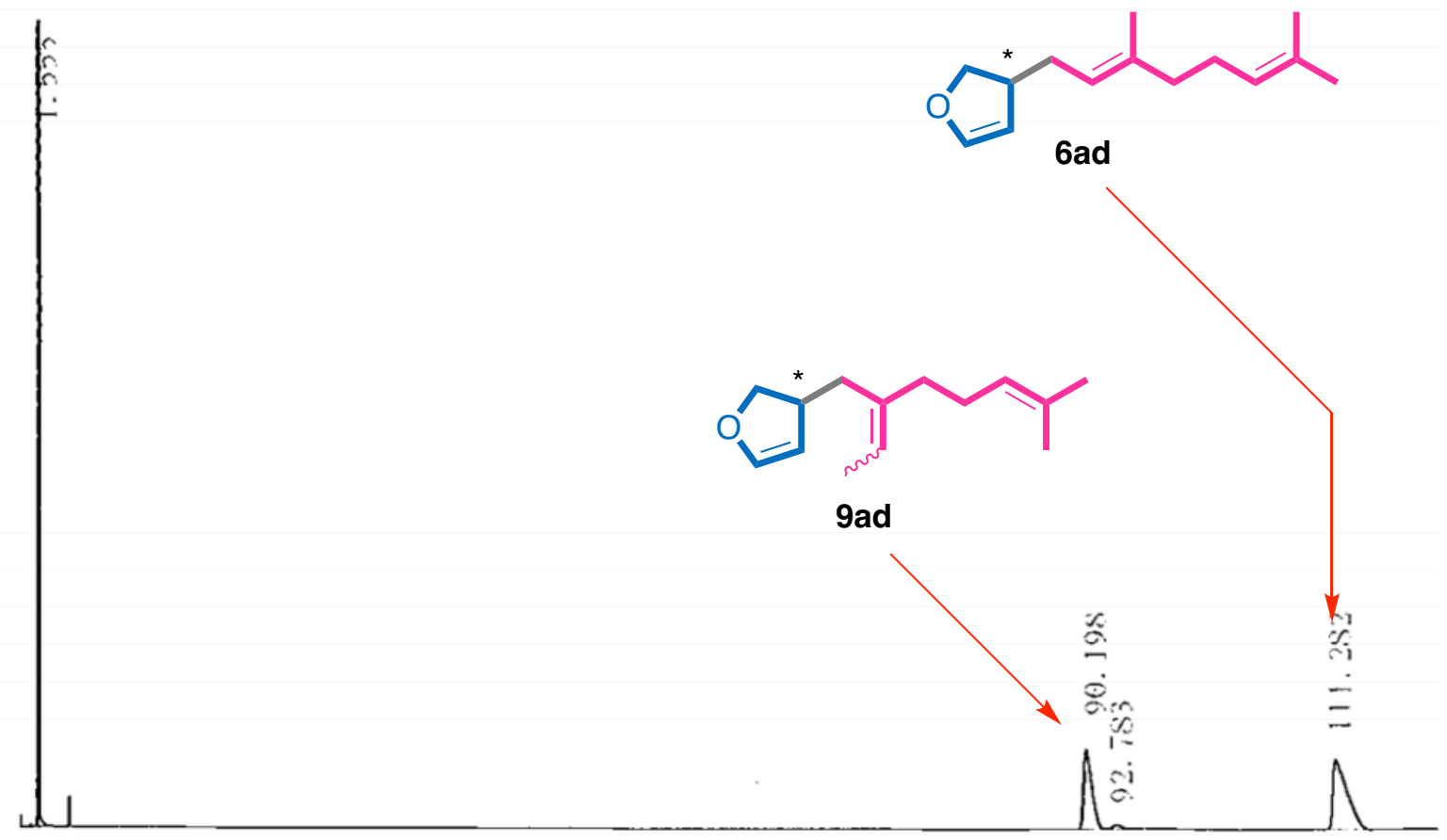

$\begin{array}{cccc}1 & 1 & 1 & 1 \\ 0.6 & 7.7 & 15.2 & =3 .\end{array}$

\$: CALCLILATION PEPOR' * *

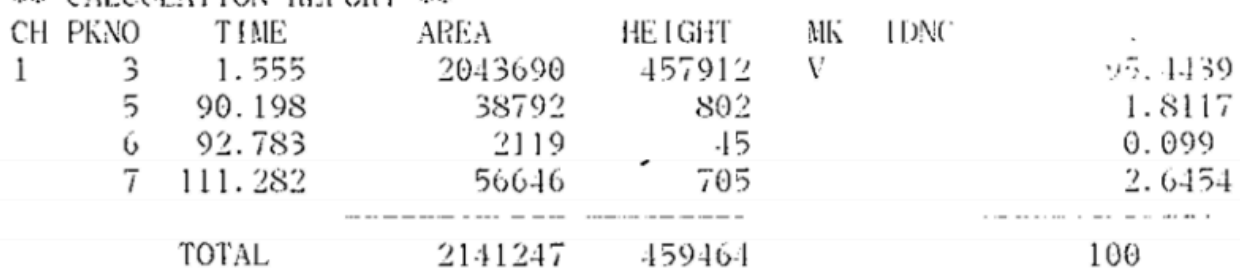

ent-9ad: $\mathrm{RT}=90.198,92.783 \mathrm{~min}$.

ee $=(38792-2119) /(38792+2119)=90 \%$ ee

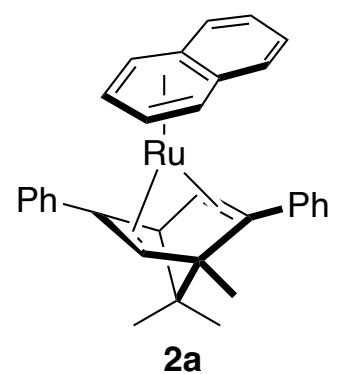

Figure S41-2. CSP-GC Chromatogram of ent-6ad and ent-9ad produced by the catalysis using $2 \mathbf{a}$ at r.t.

Conditions: Column: Rt- $\beta$ DEXsm (0.25 mmf x $30 \mathrm{~m})$, oven temp: $100^{\circ} \mathrm{C}$, linear velosity: $45 \mathrm{~cm} \mathrm{~s}^{-1}$. ent-6ad could not be separated under these conditions. 
m V

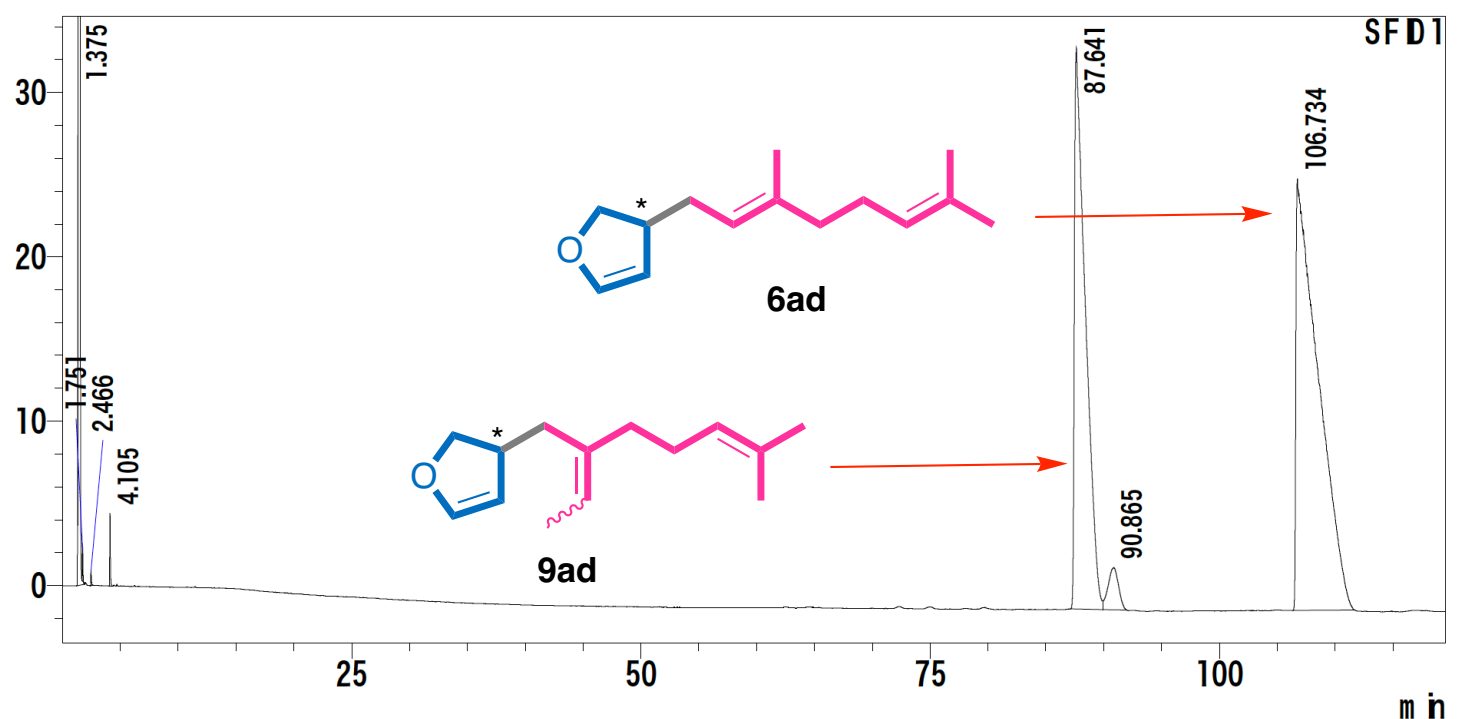

\begin{tabular}{|r|r|r|r|r|}
\hline Peak \# & $\begin{array}{l}\text { Retention } \\
\text { Time }\end{array}$ & \multicolumn{1}{l|}{ Area } & Hight & Concentration \\
\hline 1 & 87.641 & 2231860 & 34132 & 2.071 \\
\hline 2 & 90.865 & 161008 & 2566 & 0.149 \\
\hline 3 & 106.734 & 3487420 & 26227 & 3.235 \\
\hline
\end{tabular}

ent-9ad: RT $=987.641,90.865 \mathrm{~min}$.

ee $=(161008-2231860) /(161008+2231860)=86 \%$ ee

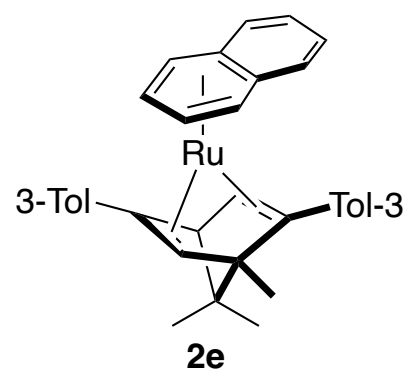

Figure S41-3. CSP-GC Chromatogram of ent-6ad and ent-9ad produced by the catalysis using $2 \mathbf{e}$ at r.t. Conditions: Column: Rt- $\beta$ DEXsm (0.25 mmf x $30 \mathrm{~m})$, oven temp: $100^{\circ} \mathrm{C}$, linear velosity: $45 \mathrm{~cm} \mathrm{~s}^{-1}$. ent-6ad could not be separated under these conditions. 
$\mathrm{mV}$

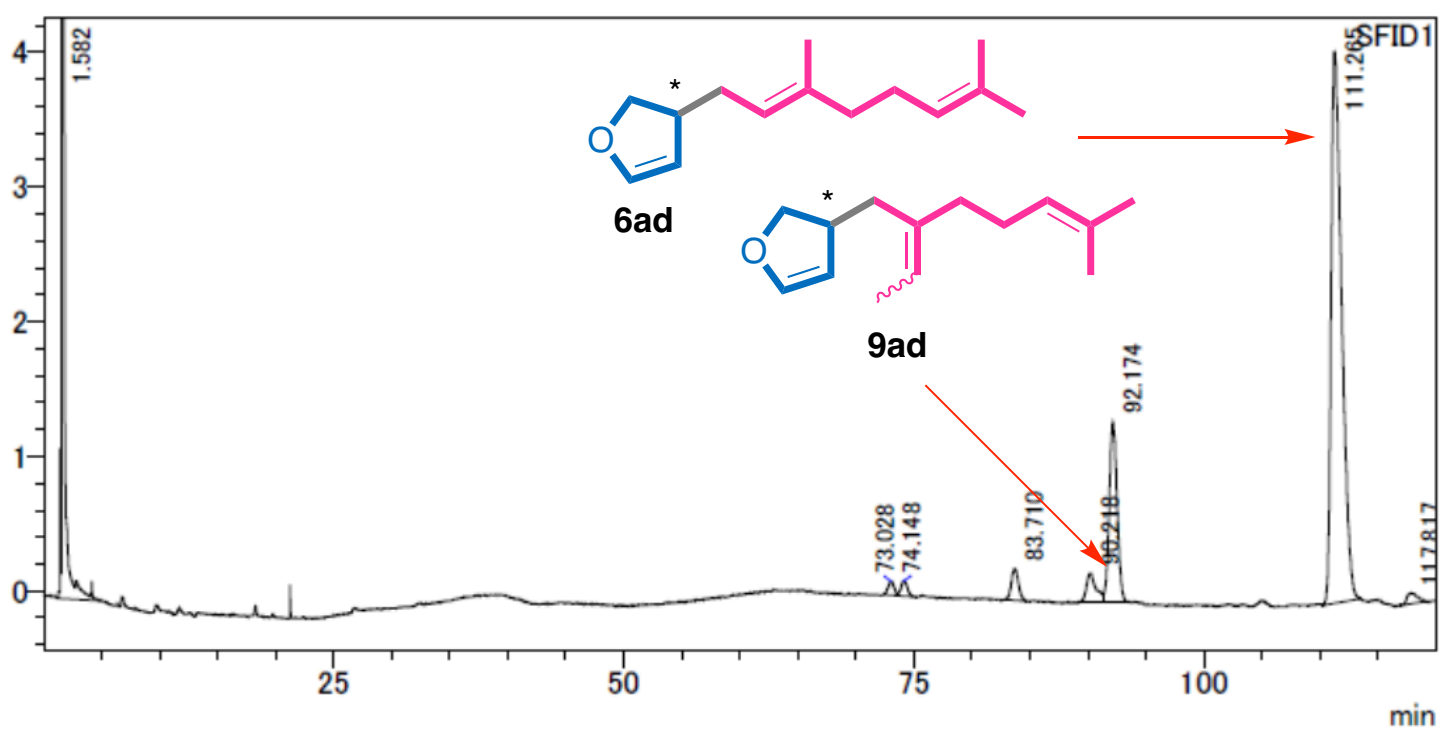

\begin{tabular}{|c|c|c|c|c|}
\hline Peak \# & $\begin{array}{l}\text { Retention } \\
\text { Time }\end{array}$ & Area & Hight & Concentration \\
\hline 1 & 90.218 & 11860 & 213 & 0.037 \\
\hline 2 & 92.174 & 63402 & 1333 & 0.196 \\
\hline 3 & 111.265 & 258513 & 4093 & 0.801 \\
\hline
\end{tabular}

ent-9ad: $\mathrm{RT}=90.218,92.174 \mathrm{~min}$.

ee $=(63402-11860) /(63402+11860)=68 \%$ ee

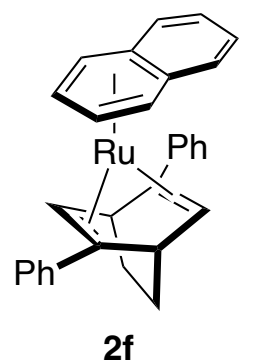

Figure S41-4. CSP-GC Chromatogram of ent-6ad and ent-9ad produced by the catalysis using $\mathbf{2 f}$ at r.t. Conditions: Column: Rt- $\beta$ DEXsm (0.25 mmf x $30 \mathrm{~m}$ ), oven temp: $100{ }^{\circ} \mathrm{C}$, linear velosity: $45 \mathrm{~cm} \mathrm{~s}^{-1}$. ent-6ad could not be separated under these conditions. 


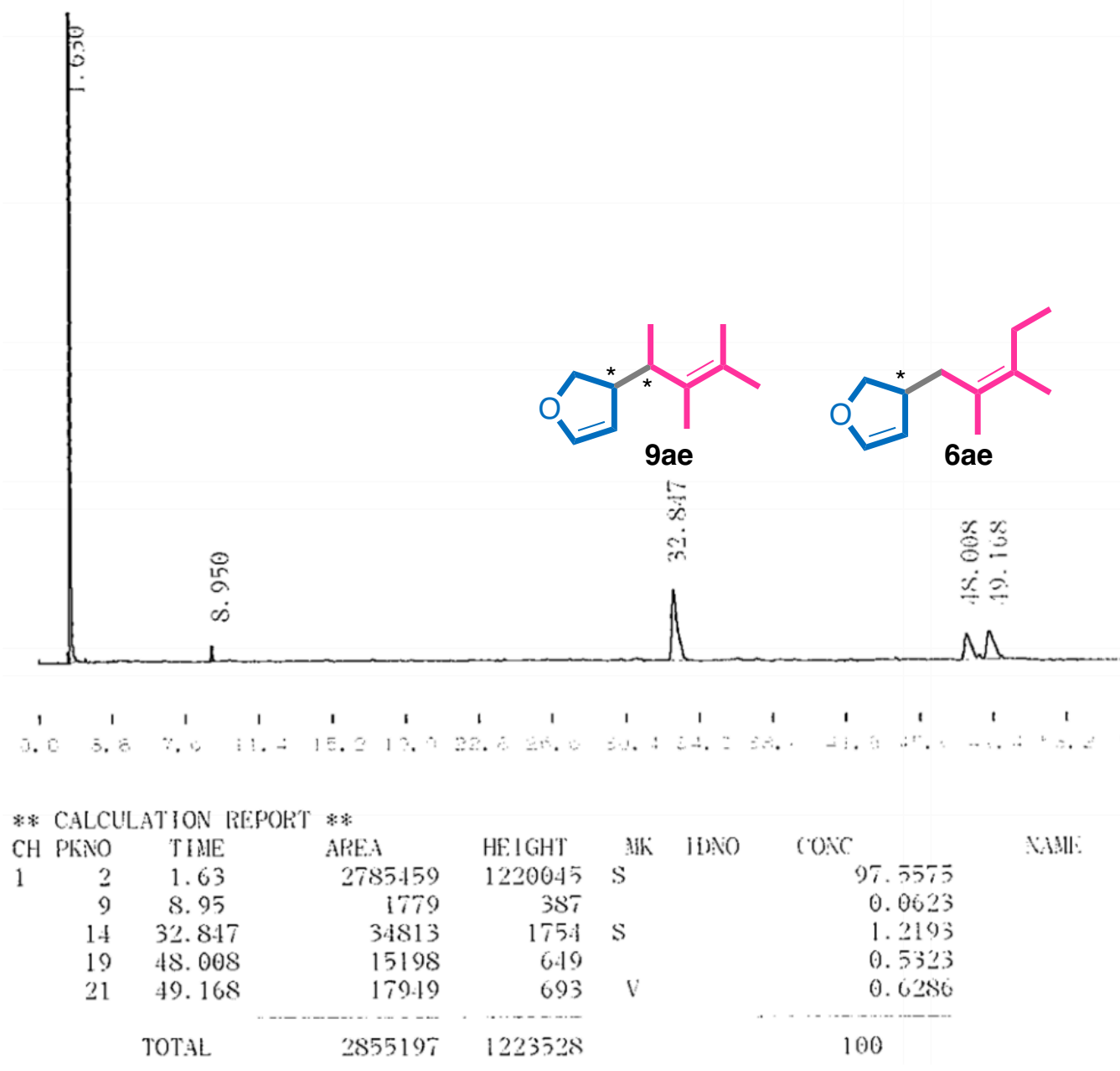

ent-6ae: $\mathrm{RT}=48.008,49.168 \mathrm{~min}$.

ee $=(17949-15198) /(17949+15198)=8 \%$ ee

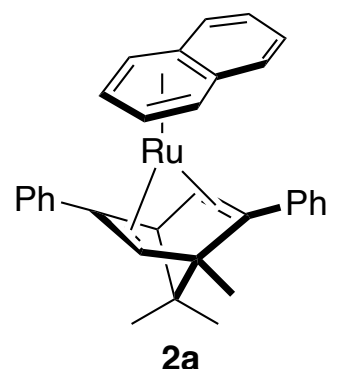

Figure S42-1. CSP-GC Chromatogram of ent-6ae and ent-9ae produced by the catalysis using $2 \mathbf{a}$ at r.t. Conditions: Column: Rt- $\beta$ DEXsm (0.25 mmf x $30 \mathrm{~m}$ ), oven temp: $80^{\circ} \mathrm{C}$, linear velosity: $40 \mathrm{~cm} \mathrm{~s}^{-1}$. Ent-6ae could not be separated under these conditions. 


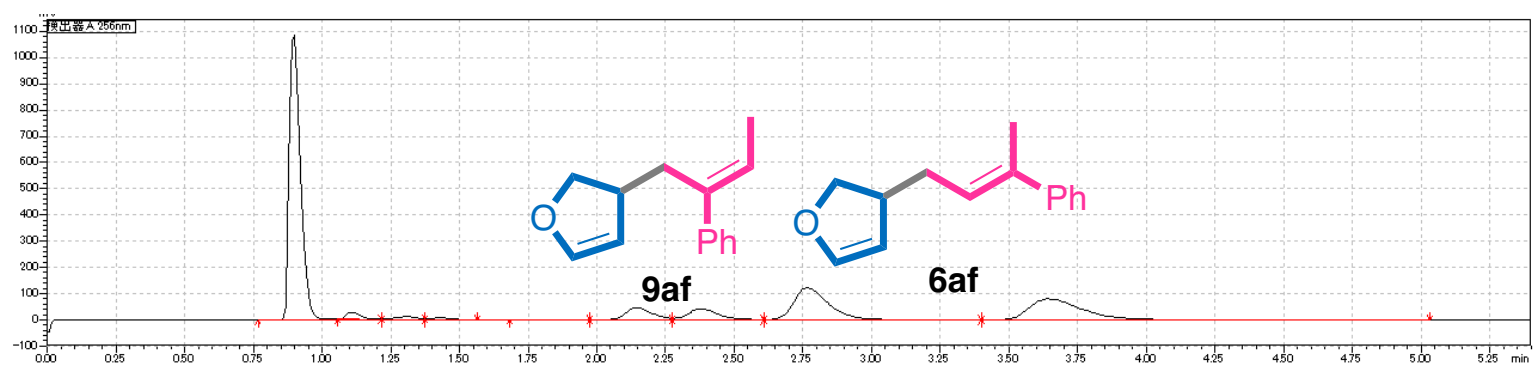

\begin{tabular}{|r|r|r|r|r|}
\hline Peak \# & \multicolumn{2}{l|}{$\begin{array}{l}\text { Retention } \\
\text { Time }\end{array}$} & \multicolumn{1}{l|}{ Hight } & Concentration \\
\hline 1 & 2.146 & 297588 & 46780 & 0 \\
\hline 2 & 2.378 & 300103 & 41047 & 0 \\
\hline 3 & 2.766 & 1065036 & 122085 & 0 \\
\hline 4 & 3.644 & 1055339 & 79708 & 0 \\
\hline
\end{tabular}

rac-9af: $\mathrm{RT}=2.146,2.378 \mathrm{~min}$

rac-6af: $\mathrm{RT}=2.766,3.644 \mathrm{~min}$

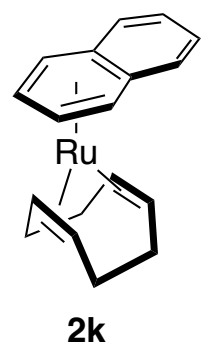

Figure S43-1. CSP-HPLC Chromatogram of rac-6af and rac-9af produced by the catalysis using $\mathbf{2 k}$ at r.t. Conditions: Column: CHIRALPAK IC (4.6 mmf x $100 \mathrm{~mm}$ ), oven temp: r.t., flow rate: $4.50 \mathrm{~mL} \mathrm{~min}{ }^{-1}$. Moving phase: hexane/isopropyl alcohol=99.9/0.1, Detector:UV@244 nm. 


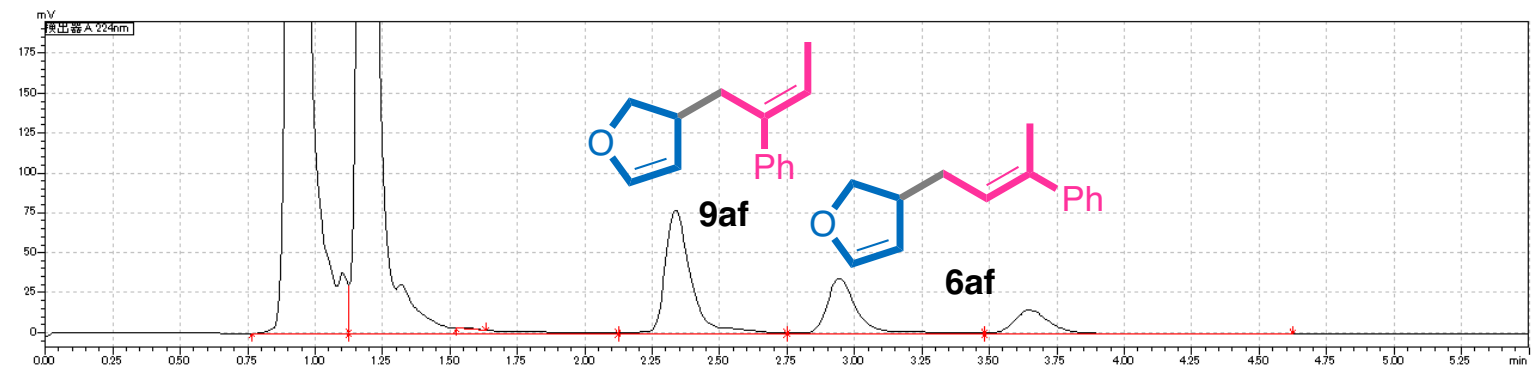

\begin{tabular}{|r|r|r|r|r|}
\hline Peak \# & \multicolumn{2}{l|}{$\begin{array}{l}\text { Retention } \\
\text { Time }\end{array}$} & \multicolumn{1}{l|}{ Area } & Concentration \\
\hline 1 & 2.336 & 478006 & 77330 & 0 \\
\hline 2 & 2.551 & 21234 & 2882 & 0 \\
\hline 3 & 2.943 & 275167 & 34434 & 0 \\
\hline 4 & 3.649 & 135922 & 14672 & 0 \\
\hline
\end{tabular}

ent-9af: $\mathrm{RT}=2.336,2.551 \mathrm{~min}$

ee $=(478006-21234) /(478006+21234)=91 \%$ ee

ent-6af: $\mathrm{RT}=2.943,3.649 \mathrm{~min}$

ee $=(275167-135922) /(27516+135922)=34 \%$ ee

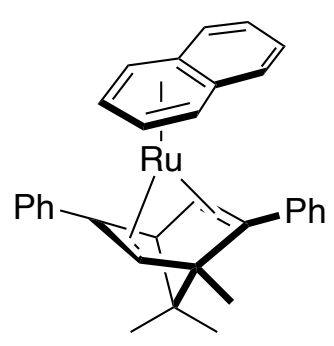

$2 a$

Figure S44-1. CSP-HPLC Chromatogram of ent-6af and ent-9af produced by the catalysis using $2 \mathbf{a}$ at 30 ${ }^{\circ} \mathrm{C}$. Conditions: Column: CHIRALPAK IC (4.6 mmf x $100 \mathrm{~mm}$ ), oven temp: r.t., flow rate: $4.50 \mathrm{~mL} \mathrm{~min}{ }^{-1}$. Moving phase: hexane/isopropyl alcohol =99.9/0.1, Detector: UV@244 nm. 


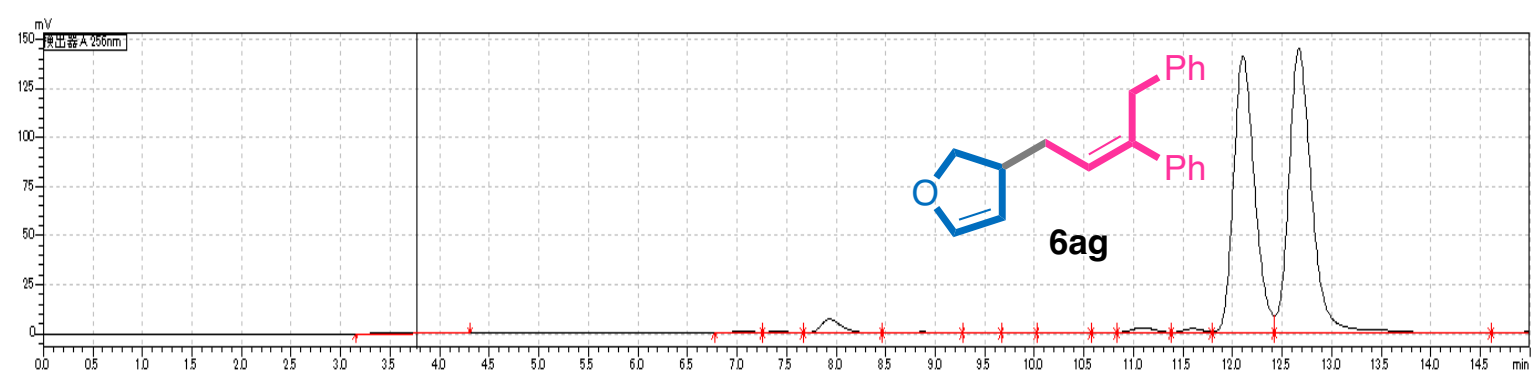

\begin{tabular}{|r|r|r|l|r|}
\hline Peak \# & \multicolumn{2}{l|}{$\begin{array}{l}\text { Retention } \\
\text { Time }\end{array}$} & Area & Hight \\
\hline 1 & 12.104 & 2081633 & & 0 \\
\hline 2 & 12.668 & 2324998 & & 0 \\
\hline
\end{tabular}

rac-6af: $\mathrm{RT}=12.104,12.668 \mathrm{~min}$

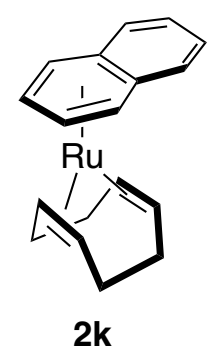

Figure S45-1. CSP-HPLC Chromatogram of rac-6ag produced by the catalysis using $\mathbf{2} \mathbf{k}$ at $30^{\circ} \mathrm{C}$.

Conditions: Column: CHIRALPAK IC (4.6 mmf x $100 \mathrm{~mm}$ ), oven temp: r.t., flow rate: $1.05 \mathrm{~mL} \mathrm{~min}^{-1}$. Moving phase: hexane/isopropyl alcohol = 99.5/0.5, Detector: UV@256 nm. 


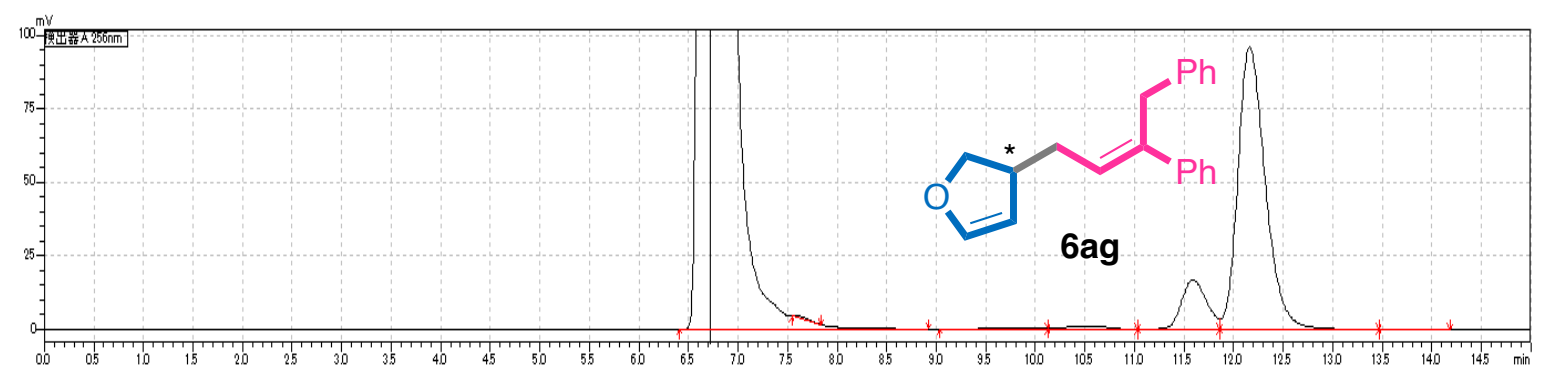

\begin{tabular}{|r|r|r|l|r|}
\hline Peak \# & \multicolumn{1}{l|}{$\begin{array}{l}\text { Retention } \\
\text { Time }\end{array}$} & Hight & Concentration \\
\hline 1 & 11.589 & 291457 & & 0 \\
\hline 2 & 12.161 & 1837919 & & 0 \\
\hline
\end{tabular}

ent-6ag: $\mathrm{RT}=11.589,12.161 \mathrm{~min}$

ee $=(1837919-291457) /(1837919+291457)=73 \%$ ee

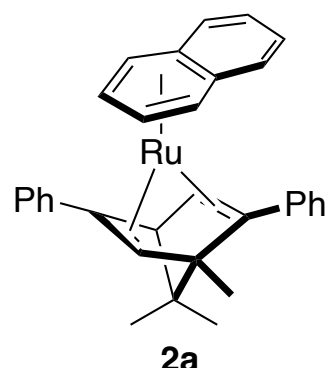

2a

Figure S46-1. CSP-HPLC Chromatogram of ent-6ag produced by the catalysis using $2 \mathbf{a}$ at r.t..

Conditions: Column: CHIRALPAK IC (4.6 mmf x $100 \mathrm{~mm}$ ), oven temp: r.t., flow rate: $1.05 \mathrm{~mL} \mathrm{~min}{ }^{-1}$. Moving phase: hexane/isopropyl alcohol =99.5/0.5, Detector: UV@256 nm. 


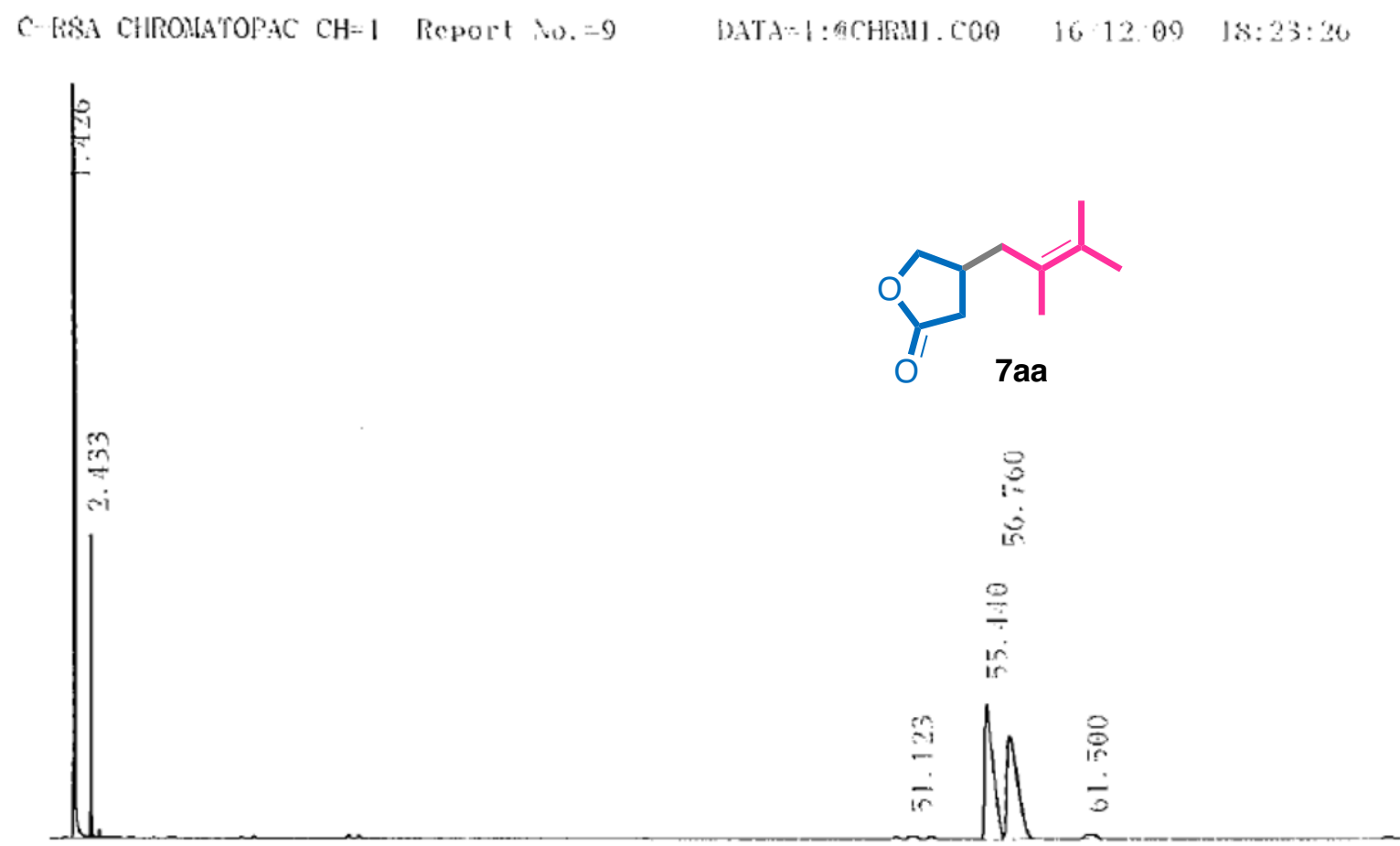

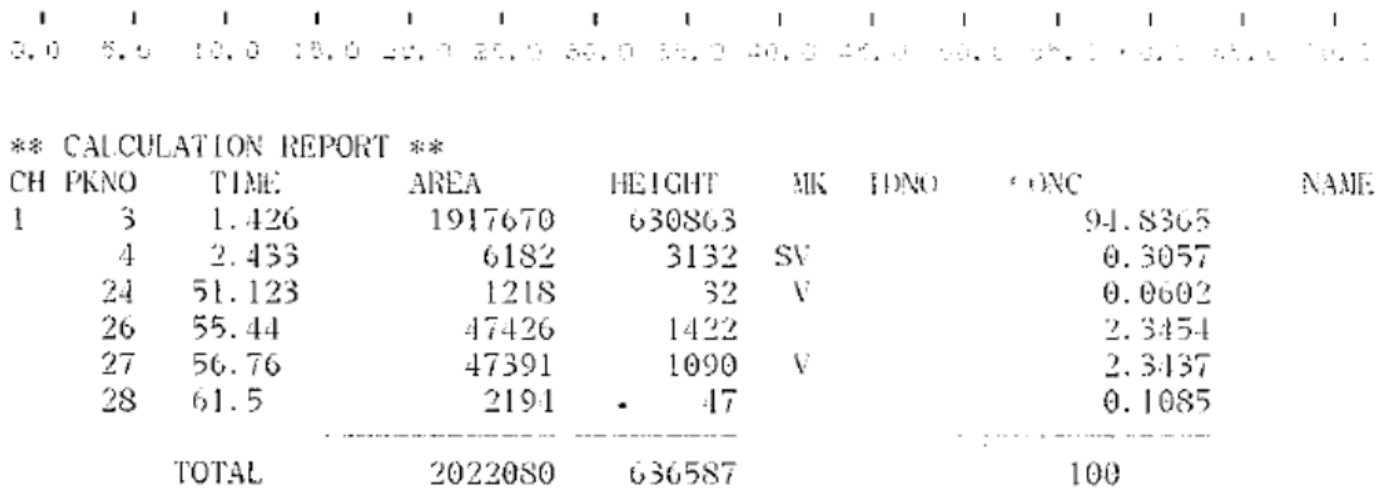

rac-7aa: $\mathrm{RT}=55.44,56.76 \mathrm{~min}$.

Figure S47-1. CSP-GC Chromatogram of rac-7aa. Conditions: Column: Rt- $\beta$ DEXsm (0.25 mmf x $30 \mathrm{~m})$, oven temp: $125^{\circ} \mathrm{C}$, linear velosity: $45 \mathrm{~cm} \mathrm{~s}^{-1}$. 

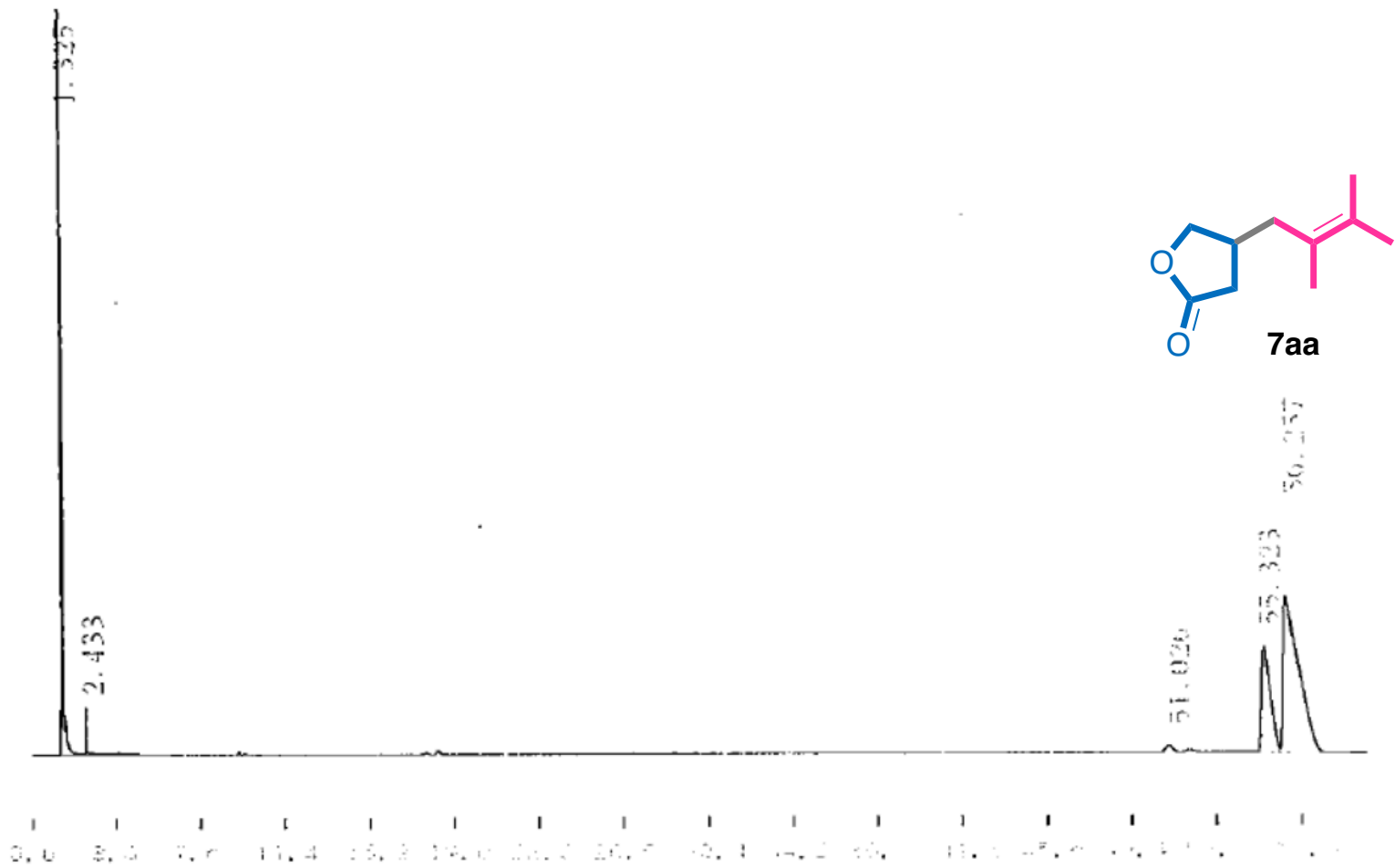

\&\% CALCLLAT!ON HELORT *

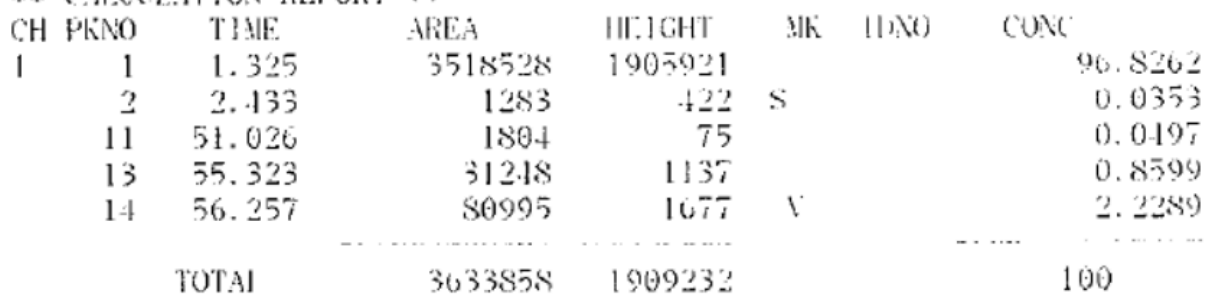

ent-7aa: RT = 55.323, 56.257min

ee $=(80995-31248) /(80995+31248)=44 \%$ ee

Figure S48-1. CSP-GC Chromatogram of ent-7aa. Conditions: Column: Rt- $\beta$ DEXsm (0.25 mmf x $30 \mathrm{~m})$, oven temp: $125^{\circ} \mathrm{C}$, linear velosity: $45 \mathrm{~cm} \mathrm{~s}^{-1}$. 
$\mathrm{mV}$

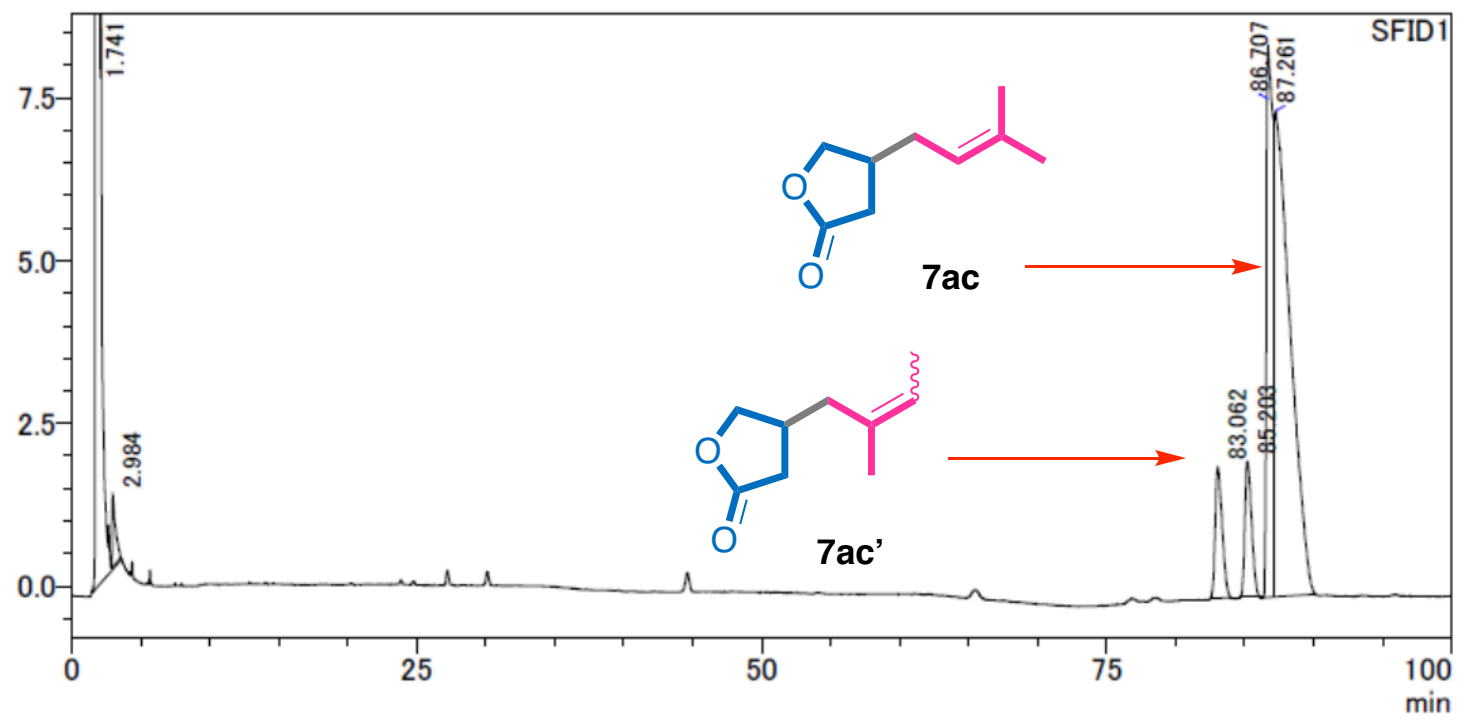

\begin{tabular}{|c|c|c|c|c|}
\hline Peak \# & $\begin{array}{l}\text { Retention } \\
\text { Time }\end{array}$ & Area & Hight & Concentration \\
\hline 1 & 83.062 & 70598 & 2018 & 0.029 \\
\hline 2 & 85.203 & 70944 & 2082 & 0.029 \\
\hline 3 & 86.707 & 266514 & 8480 & 0.108 \\
\hline 4 & 87.261 & 606557 & 7457 & 0.246 \\
\hline
\end{tabular}

rac-7ac': $\mathrm{RT}=83.062,85.203 \mathrm{~min}$.

Figure S49-1. CSP-GC Chromatogram of rac-7ac'. Conditions: Column: Rt- $\beta$ DEXsm $(0.25 \mathrm{mmf} \times 30 \mathrm{~m})$, oven temp: $110^{\circ} \mathrm{C}$, linear velosity: $40 \mathrm{~cm} \mathrm{~s}^{-1}$. Rac-7ac could not be separated under these conditions. 
$\mathrm{mV}$

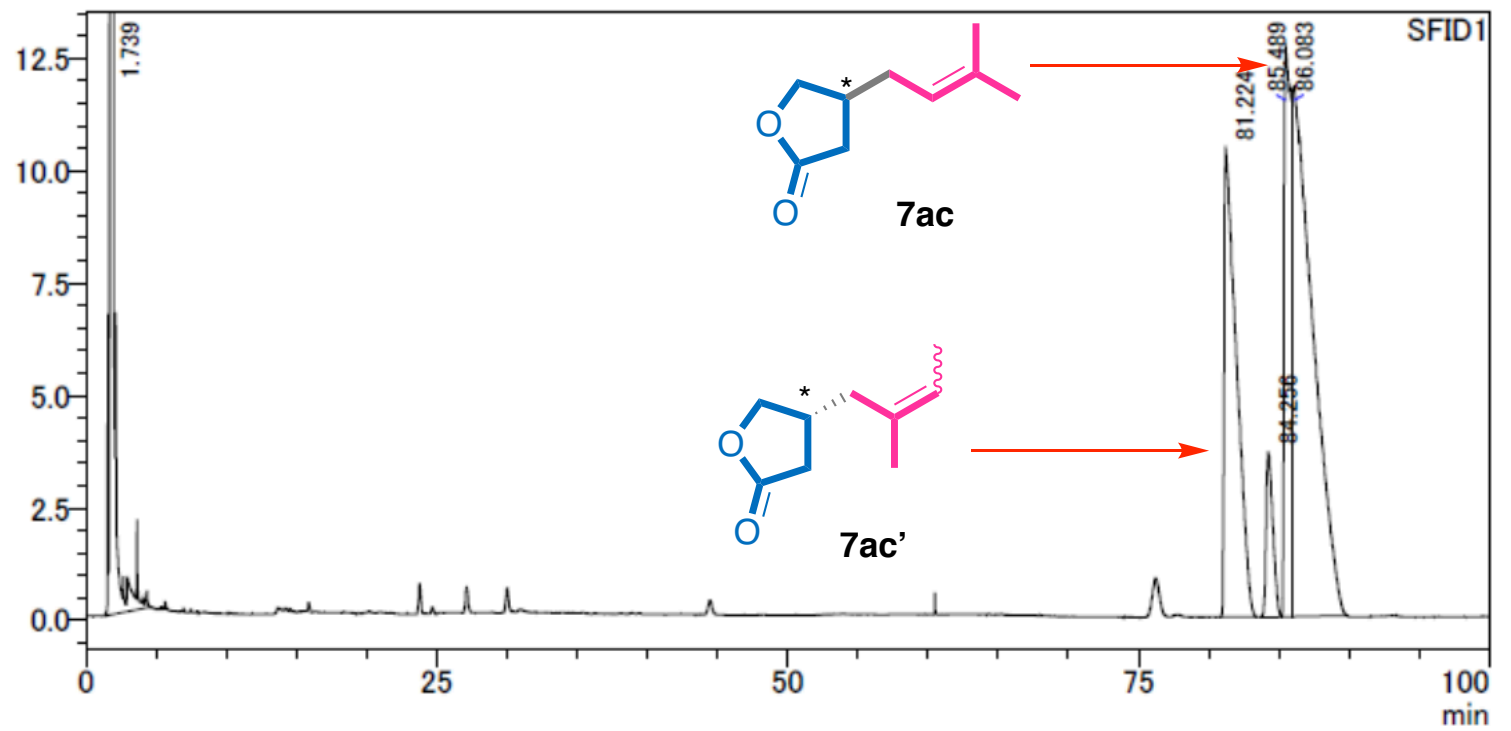

\begin{tabular}{|r|r|r|r|r|}
\hline Peak \# & \multicolumn{1}{l|}{$\begin{array}{l}\text { Retention } \\
\text { Time }\end{array}$} & \multicolumn{1}{l|}{ Area } & Concentration \\
\hline 1 & 81.224 & 632414 & 10434 & 0.227 \\
\hline 2 & 84.256 & 123572 & 3658 & 0.044 \\
\hline 3 & 85.489 & 395169 & 12714 & 0.142 \\
\hline 4 & 86.083 & 1232907 & 11784 & 0.442 \\
\hline
\end{tabular}

ent-7ac': RT $=81.224,84.256 \mathrm{~min}$.

ee $=(632414-123572) /(632414+123572)=67 \%$ ee

Figure S50-1. CSP-GC Chromatogram of ent-7ac'. Conditions: Column: Rt- $\beta$ DEXsm (0.25 mmf x $30 \mathrm{~m})$, oven temp: $110^{\circ} \mathrm{C}$, linear velosity: $40 \mathrm{~cm} \mathrm{~s}^{-1}$. Ent-7ac could not be separated under these conditions. 


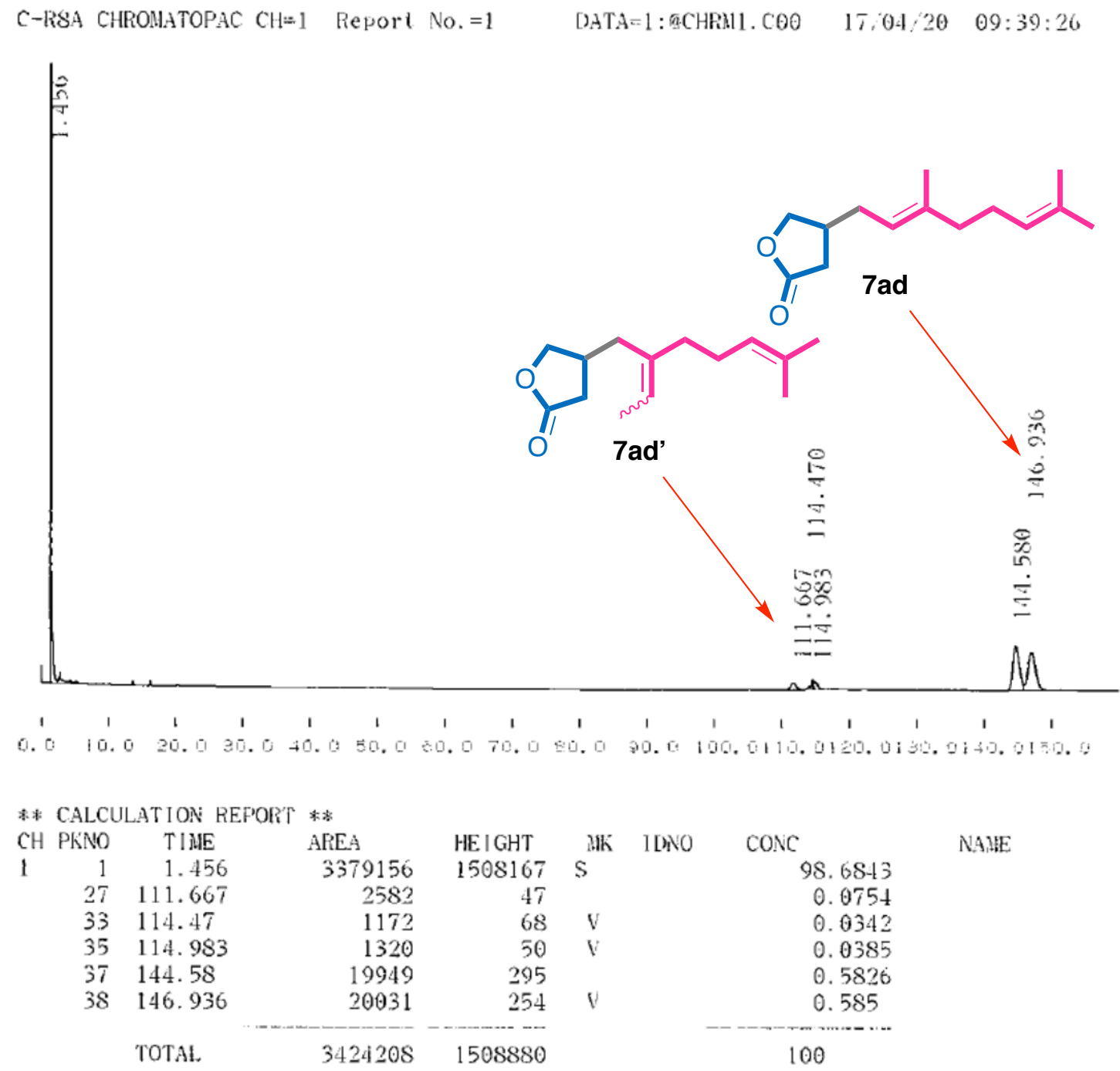

rac-7ad': RT $=111.667,114.470 \mathrm{~min}$.

rac-7ad': $\mathrm{RT}=144.580,146.936 \mathrm{~min}$.

Figure S51-1. CSP-GC Chromatogram of rac-7ad and rac-7ad'. Conditions: Column: Rt- $\gamma$ DEXsa $(0.25$ $\mathrm{mmf} \times 30 \mathrm{~m}$ ), oven temp: $160^{\circ} \mathrm{C}$, linear velosity: $40 \mathrm{~cm} \mathrm{~s}^{-1}$. 


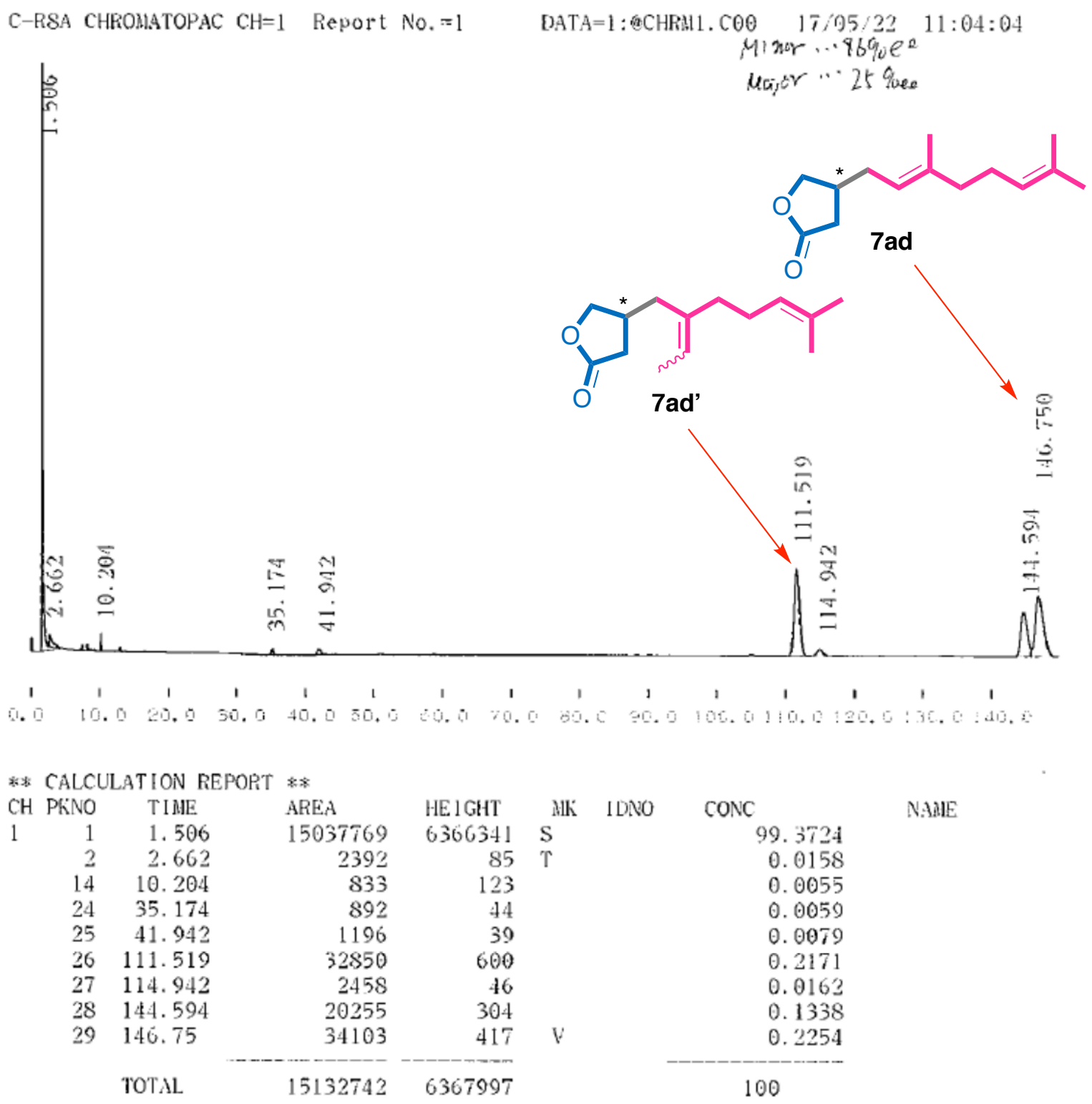

ent-7ad': RT = 111.519, $114.942 \mathrm{~min}$.

ee $=(32850-2458)(32850+2458)=86 \%$ ee

ent-7ad: $\mathrm{RT}=144.594146 .750 \mathrm{~min}$.

ee $=(34103-20255)(34103+20255)=25 \%$ ee

Figure S52-1. CSP-GC Chromatogram of ent-7ad and ent-7ad' derived from ent-6ad and ent-9ad catalyzed by 2a at r.t.. Conditions: Column: Rt- $\gamma$ DEXsa $(0.25 \mathrm{mmf}$ x $30 \mathrm{~m})$, oven temp: $160^{\circ} \mathrm{C}$, linear velosity: $40 \mathrm{~cm} \mathrm{~s}^{-1}$. 


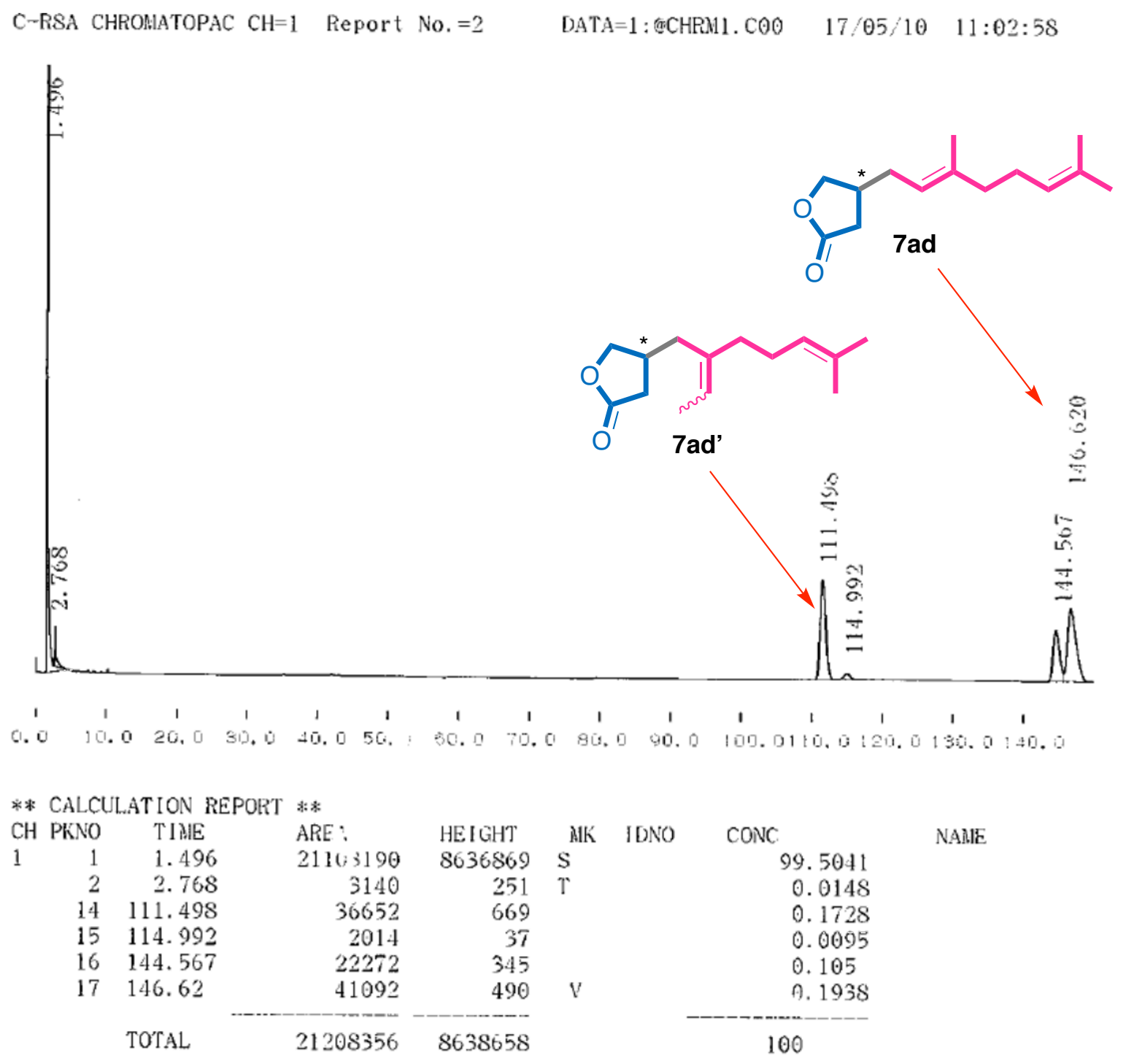

ent-7ad': RT = 111.498, $114.992 \mathrm{~min}$.

ee $=(36652-2014)(36652+2014)=90 \%$ ee

ent-7ad: $\mathrm{RT}=144.576146 .620 \mathrm{~min}$.

ee $=(41092-22272)(41092+22272)=30 \%$ ee

Figure S52-2. CSP-GC Chromatogram of ent-7ad and ent-7ad' derived from ent-6ad and ent-9ad catalyzed by $2 \mathrm{a}$ at $0{ }^{\circ} \mathrm{C}$. Conditions: Column: Rt- $\gamma \mathrm{DEXsa}(0.25 \mathrm{mmf} \times 30 \mathrm{~m})$, oven temp: $160{ }^{\circ} \mathrm{C}$, linear velosity: $40 \mathrm{~cm} \mathrm{~s}^{-1}$. 


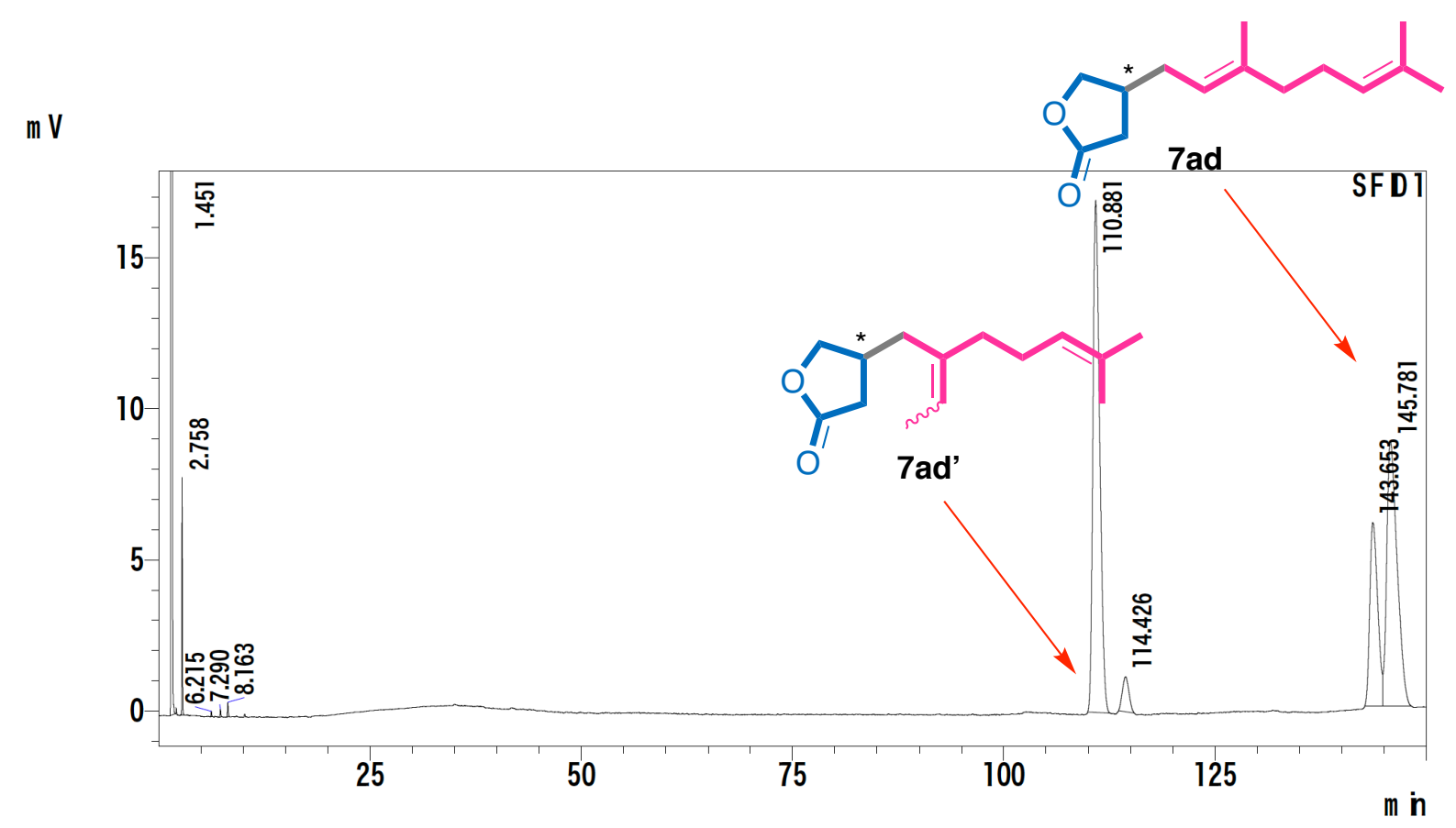

\begin{tabular}{|r|r|r|r|r|}
\hline Peak \# & \multicolumn{1}{l|}{$\begin{array}{l}\text { Retention } \\
\text { Time }\end{array}$} & \multicolumn{1}{l|}{ Hrea } & Concentration \\
\hline 1 & 110.881 & 941711 & 16956 & 0.894 \\
\hline 2 & 114.426 & 62292 & 1162 & 0.059 \\
\hline 3 & 143.653 & 393219 & 6071 & 0.373 \\
\hline 4 & 145.781 & 747348 & 8735 & 0.71 \\
\hline
\end{tabular}

ent-7ad': RT = 110.881, 114.426 min.

$\mathrm{ee}=(941711-62292)(941711+62292)=87 \%$ ee

ent-7ad: $\mathrm{RT}=143.653,145.781 \mathrm{~min}$.

$\mathrm{ee}=(747348-393219)(747348+393219)=31 \%$ ee

Figure S52-3. CSP-GC Chromatogram of ent-7ad and ent-7ad' derived from ent-6ad and ent-9ad catalyzed by $2 \mathrm{e}$ at r.t. Conditions: Column: Rt- $\gamma$ DEXsa $(0.25 \mathrm{mmf} \times 30 \mathrm{~m})$, oven temp: $160{ }^{\circ} \mathrm{C}$, linear velosity: $40 \mathrm{~cm} \mathrm{~s}^{-1}$. 


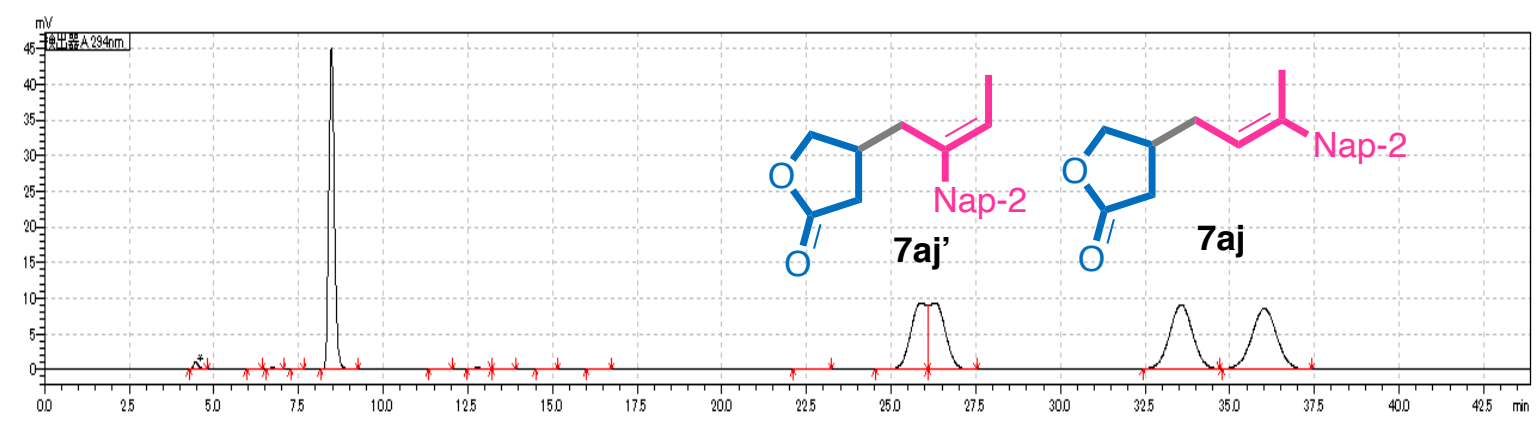

\begin{tabular}{|r|r|r|r|r|}
\hline Peak \# & \multicolumn{1}{|l|}{$\begin{array}{l}\text { Retention } \\
\text { Time }\end{array}$} & \multicolumn{1}{l|}{ Area } & Hight & Concentration \\
\hline 1 & 25.882 & 307725 & 9297 & 0 \\
\hline 2 & 26.287 & 313979 & 9315 & 0 \\
\hline 3 & 33.561 & 419107 & 8963 & 0 \\
\hline 4 & 36.014 & 434723 & 8488 & 0 \\
\hline
\end{tabular}

rac-7aj': $\mathrm{RT}=25.882,26.287 \mathrm{~min}$.

rac-7aj': RT $=33.561,36.014 \mathrm{~min}$.

Figure S53-1. CSP-HPLC Chromatogram of rac-7aj and rac-7aj' derived from rac-6aj and rac-9aj catalyzed by $\mathbf{2 k}$ at r.t. Conditions: Column: CHIRALPAK IC ( $4.6 \mathrm{mmf} \times 100 \mathrm{~mm}$ ), oven temp: r.t., flow rate: $0.800 \mathrm{~mL} \mathrm{~min}{ }^{-1}$. Moving phase: hexane/isopropyl alcohol=75.0/25.0, Detector: UV@294 nm. 


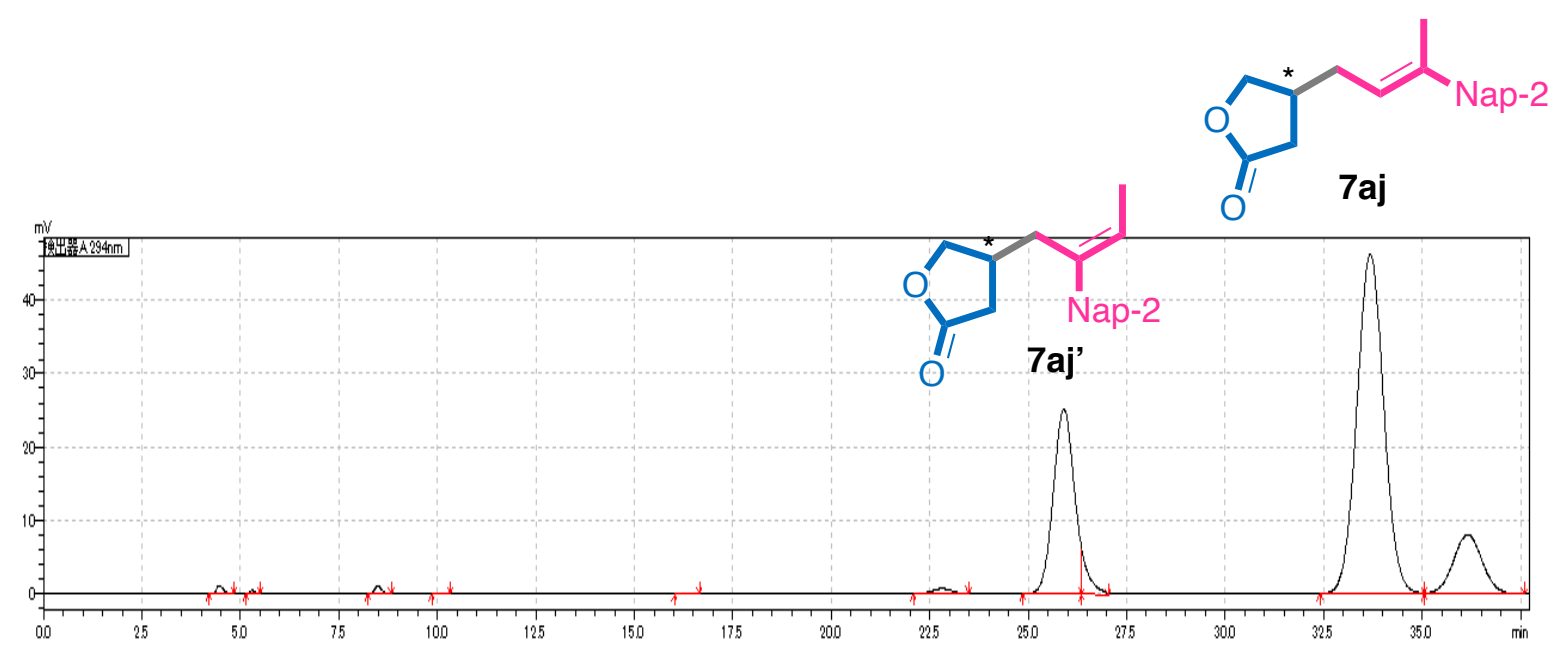

\begin{tabular}{|r|r|r|r|r|}
\hline Peak \# & $\begin{array}{l}\text { Retention } \\
\text { Time }\end{array}$ & \multicolumn{1}{l|}{ Area } & Hight \\
\hline 1 & 25.903 & 980595 & 25122 & 0 \\
\hline 2 & 33.685 & 2201096 & 46200 & 0 \\
\hline 3 & 36.158 & 413234 & 8062 & 0 \\
\hline
\end{tabular}

ent-7aj': RT = $25.903 \mathrm{~min}$.

The enantiomeric excess of ent-7aj' could not be calculated because of incomplete separation. ent-7aj: RT = 33.685, 36.158 min.

ee $=(2201096-413234)(2201096+413234)=68 \%$ ee

Figure S53-2. CSP-HPLC Chromatogram of ent-7aj and ent-7aj' derived from ent-6aj and ent-9aj catalyzed by $2 \mathrm{a}$ at $30^{\circ} \mathrm{C}$. Conditions: Column: CHIRALPAK IC ( $4.6 \mathrm{mmf}$ x $\left.100 \mathrm{~mm}\right)$, oven temp: r.t., flow rate: $0.800 \mathrm{~mL} \mathrm{~min}{ }^{-1}$. Moving phase: hexane/isopropyl alcohol=75.0/25.0, Detector: UV@294 nm. 


\section{DFT Calculations}

Cartesian coordinates of all molecules, intermediates, and transition states are uploaded in the xyz file.

\section{Complex 2a}

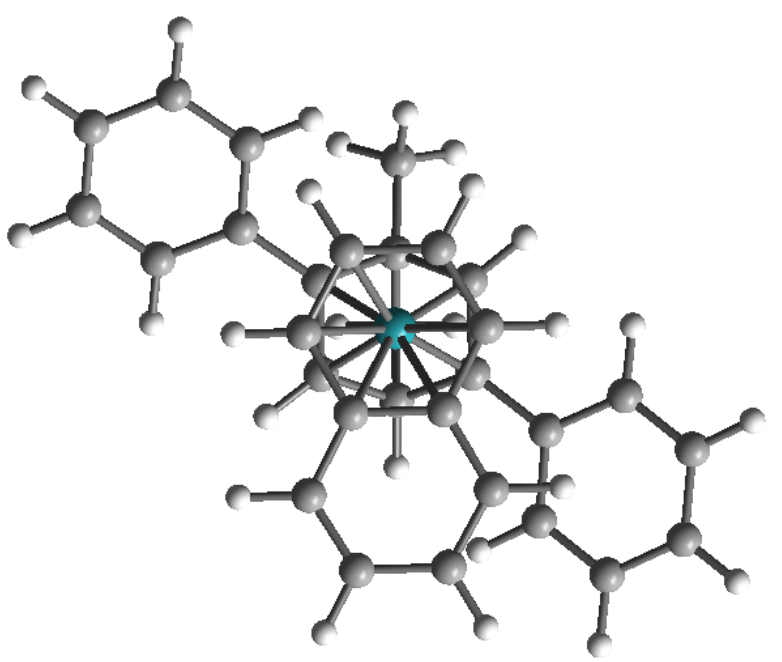

Path $(S)-6 a a$

Int A ((S)-6aa): $0.00 \mathrm{kcal} \mathrm{mol}^{-1}$

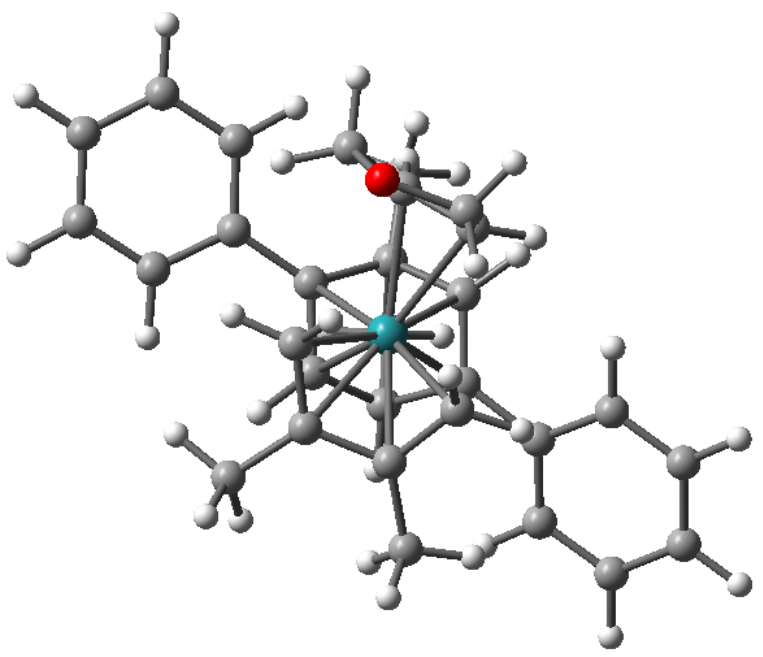


TS1 ((S)-6aa): $14.37 \mathrm{kcal} \mathrm{mol}^{-1}$

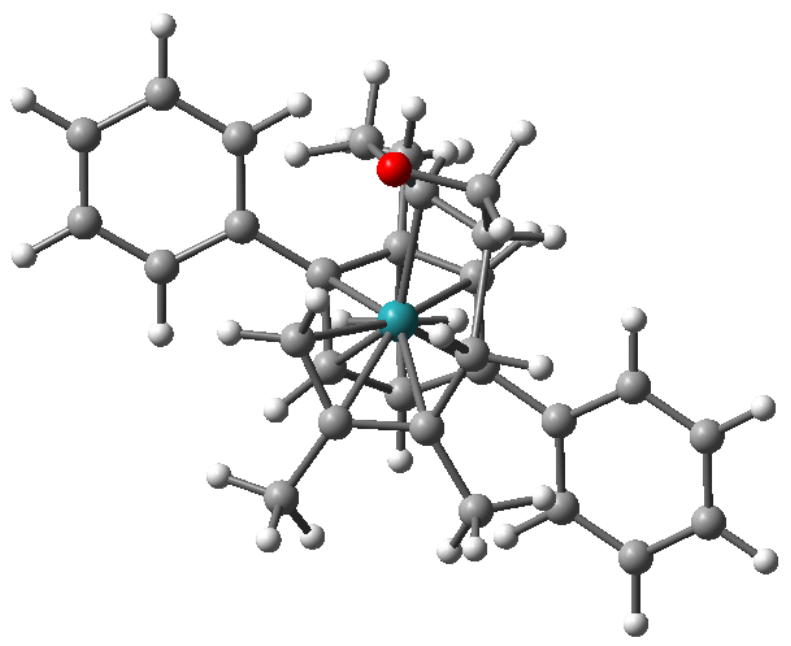

Int B ((S)-6aa): 5.79 kcal mol $^{-1}$

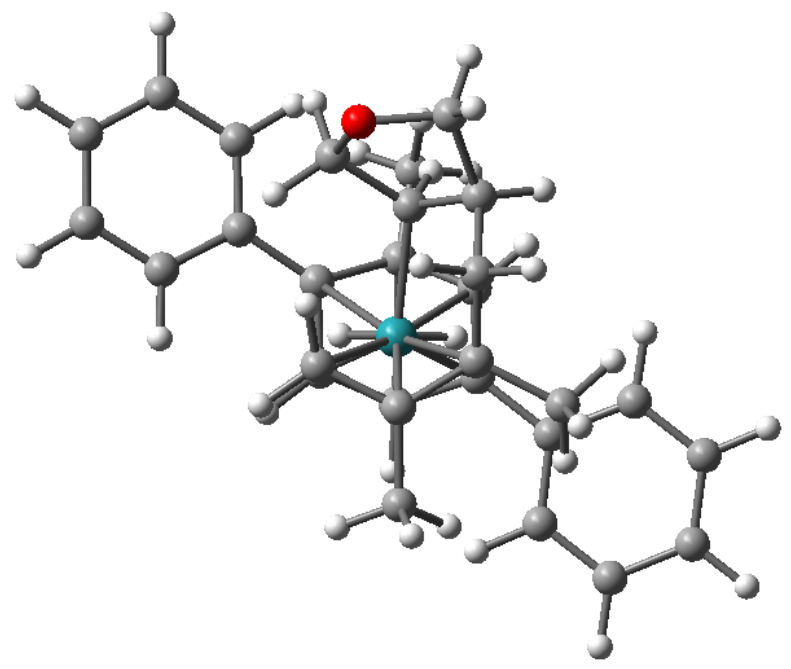

Int B' ((S)-6aa): 0.77 kcal mol'-1

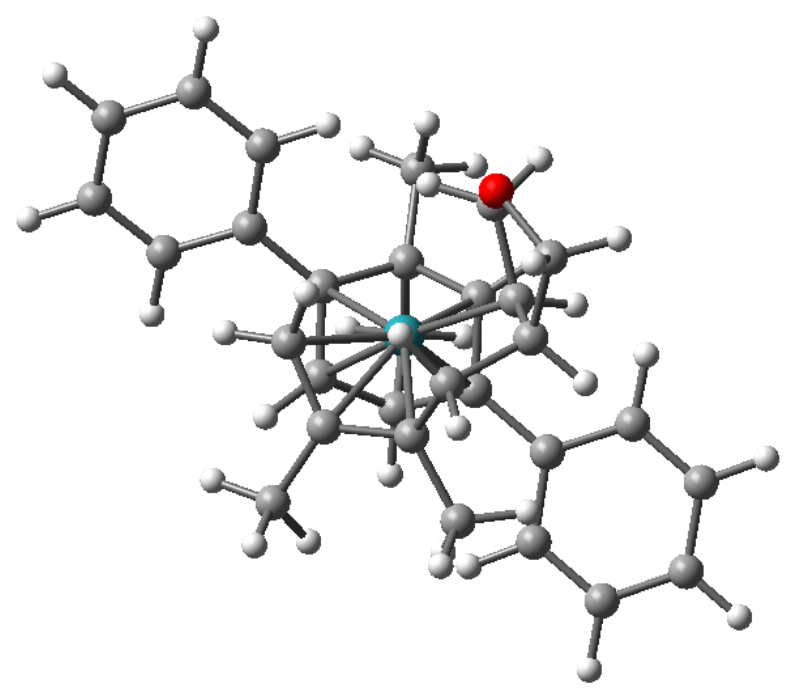

S- 96 
TS2 ((S)-6aa): $9.27 \mathrm{kcal} \mathrm{mol}^{-1}$

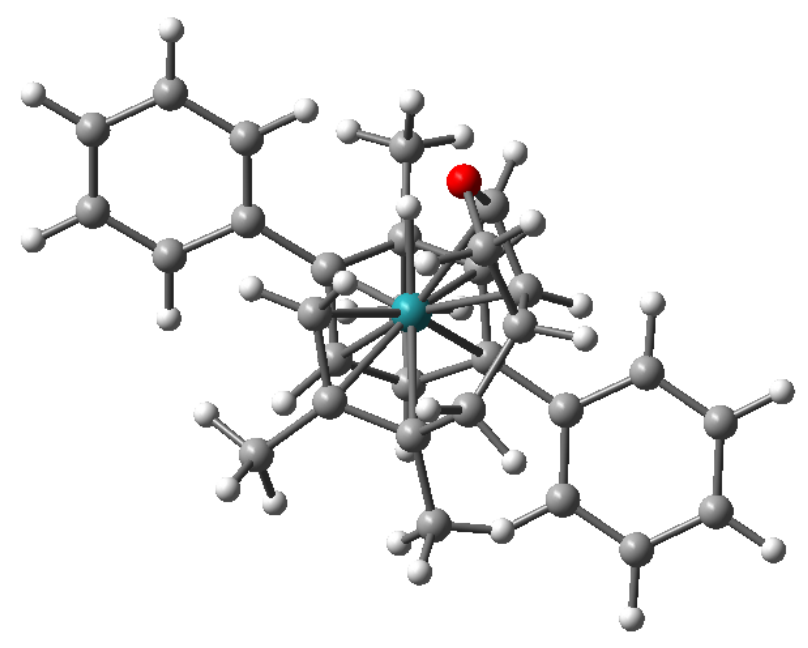

Int C ((S)-6aa): $4.57 \mathrm{kcal} \mathrm{mol}^{-1}$

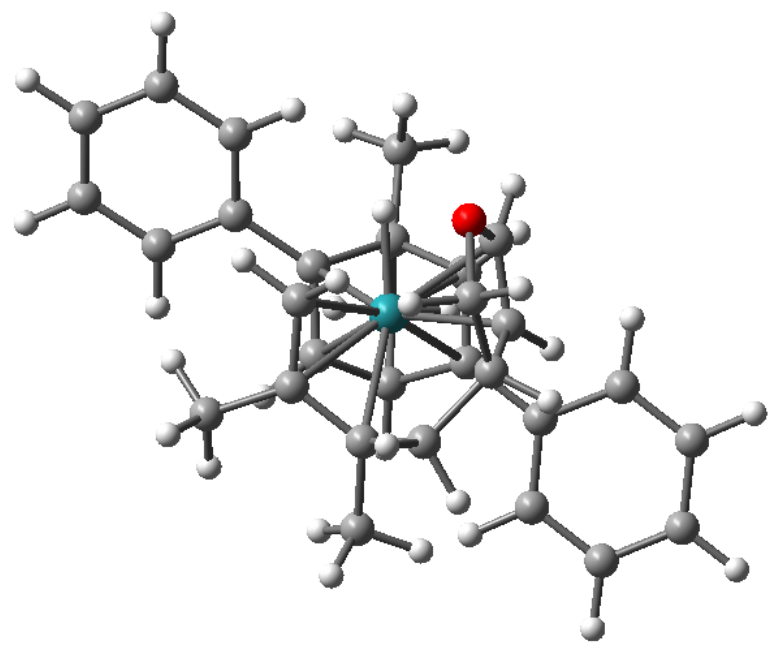

TS3 ((S)-6aa): $13.47 \mathrm{kcal} \mathrm{mol}^{-1}$

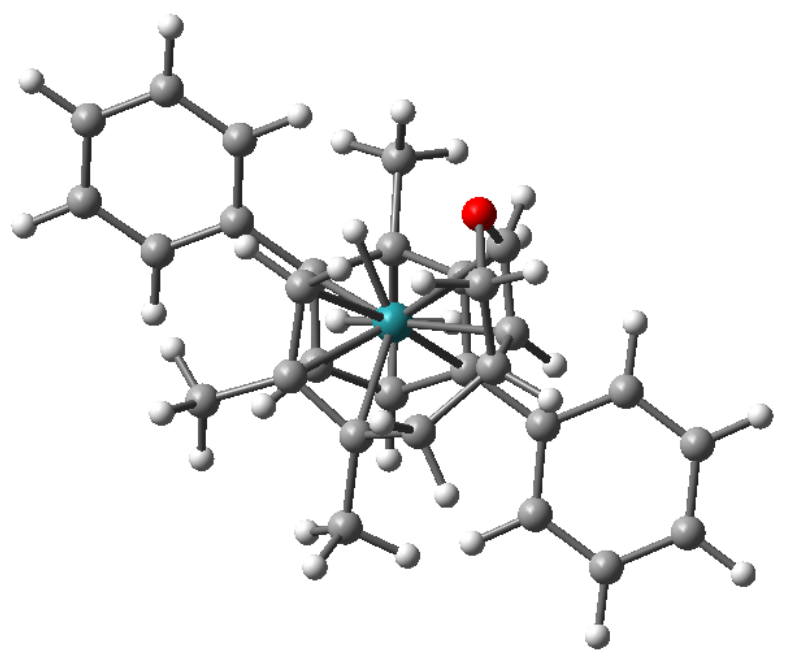

S- 97 
Int D ((S)-6aa): $-\mathbf{5 . 9 9} \mathrm{kcal} \mathrm{mol}^{-1}$

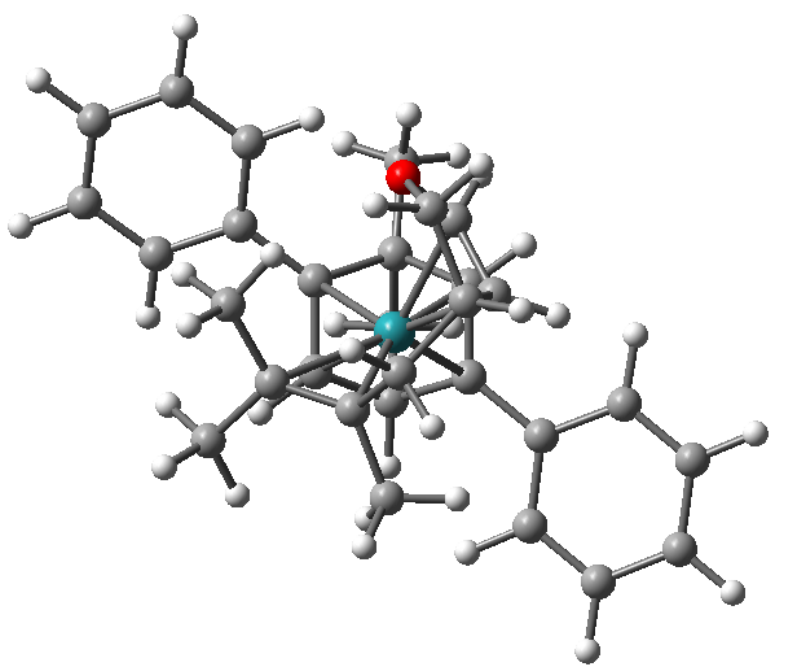

S- 98 
Path $(R)$-6aa

Int A ((R)-6aa): $0.53 \mathrm{kcal} \mathrm{mol}^{-1}$

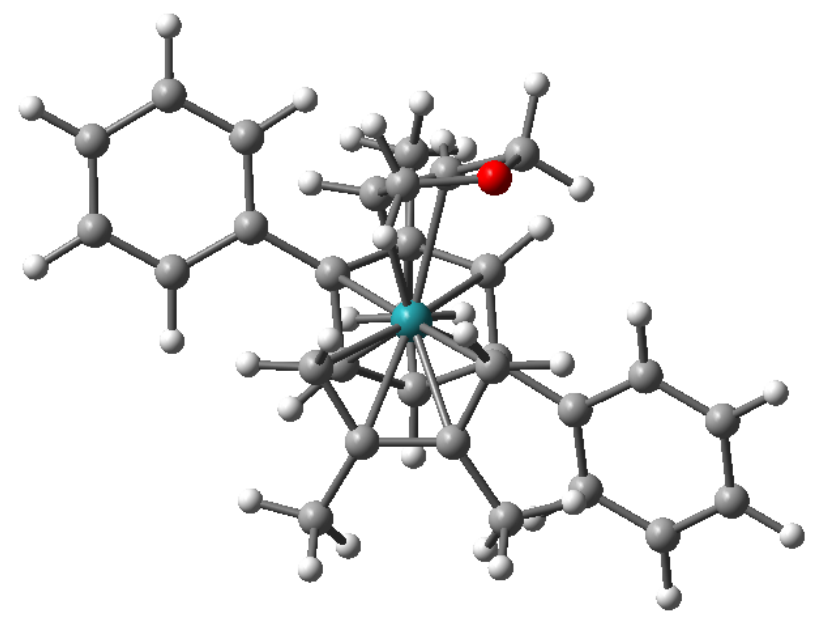

TS1 ((R)-6aa): $16.02 \mathrm{kcal} \mathrm{mol}^{-1}$

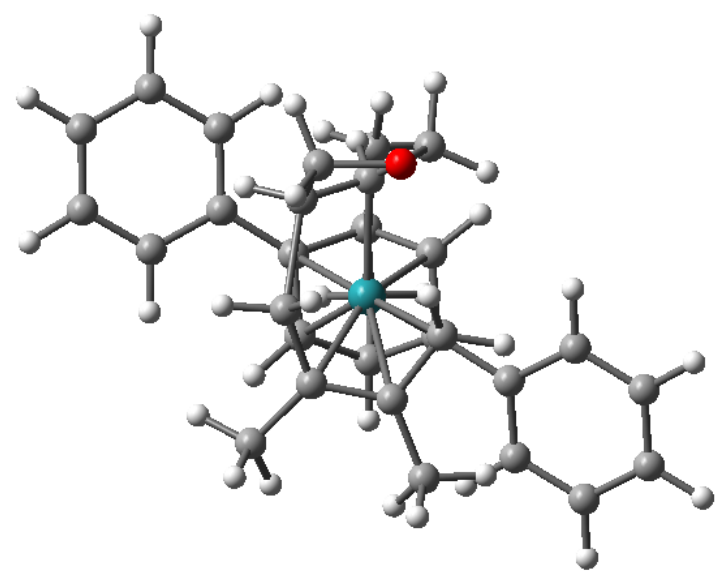

Int B ((R)-6aa): $2.13 \mathrm{kcal} \mathrm{mol}^{-1}$

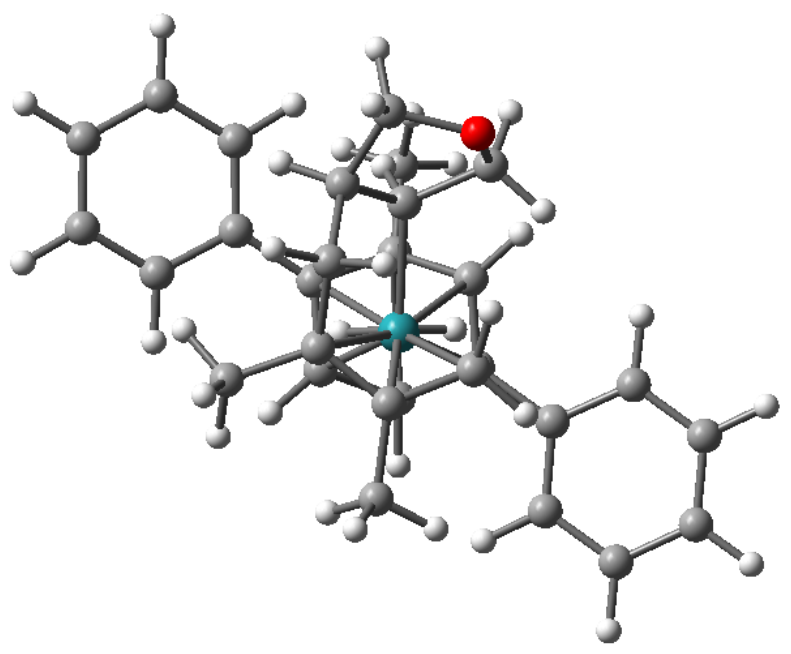

S- 99 
Int B' ((R)-6aa): $1.89 \mathrm{kcal} \mathrm{mol}^{-1}$

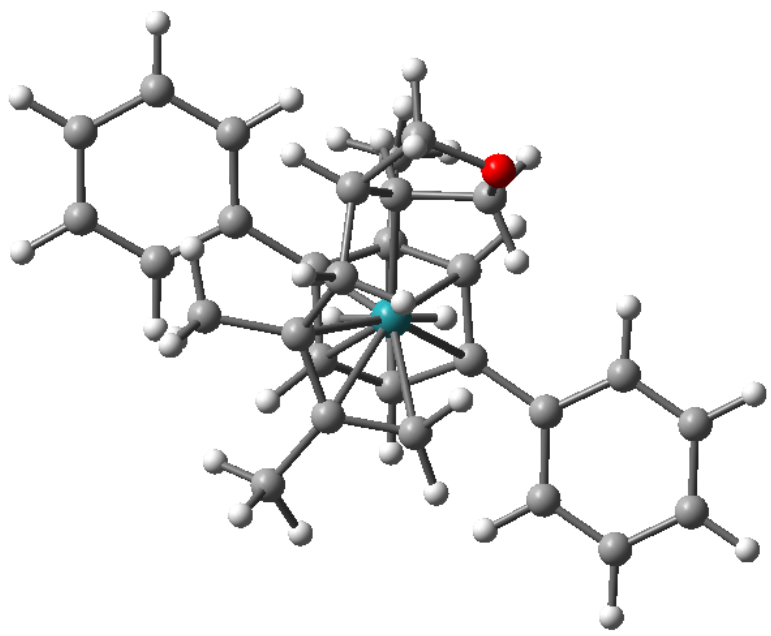

TS2 ((R)-6aa): $16.57 \mathrm{kcal} \mathrm{mol}^{-1}$

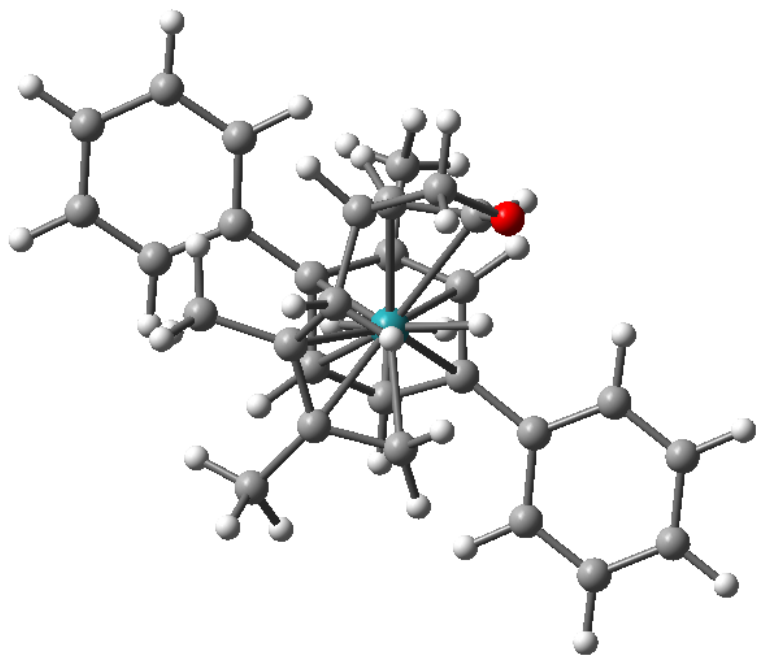

Int C (( $R)-6 a a): 10.15 \mathrm{kcal} \mathrm{mol}^{-1}$

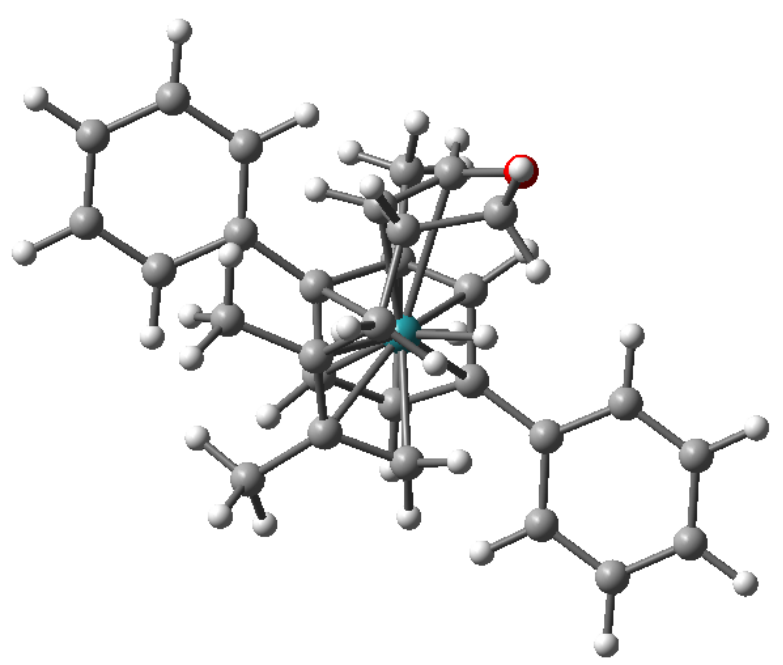

S- 100 
TS3 ((R)-6aa): $14.27 \mathrm{kcal} \mathrm{mol}^{-1}$

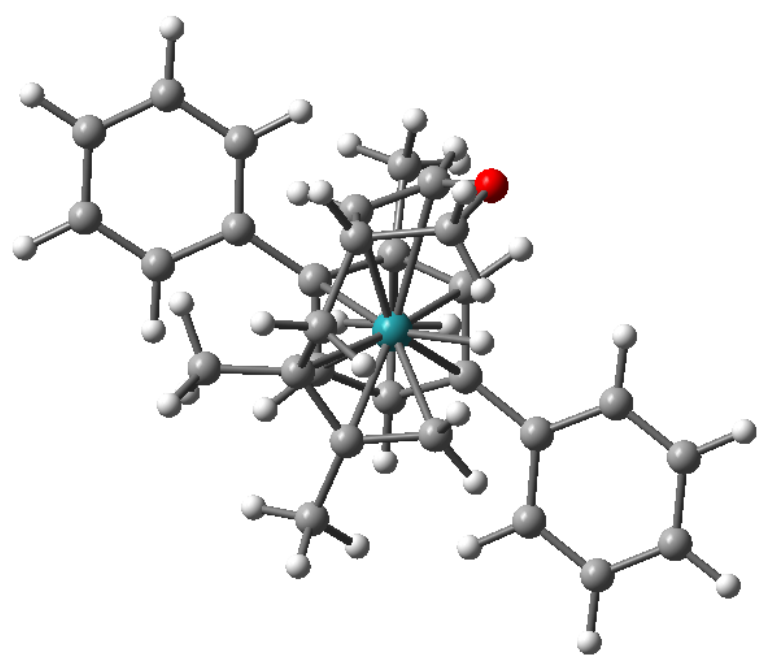

Int D (( $R)-6 \mathrm{aa}):-1.61 \mathrm{kcal}^{\mathrm{mol}} \mathbf{1}^{-1}$

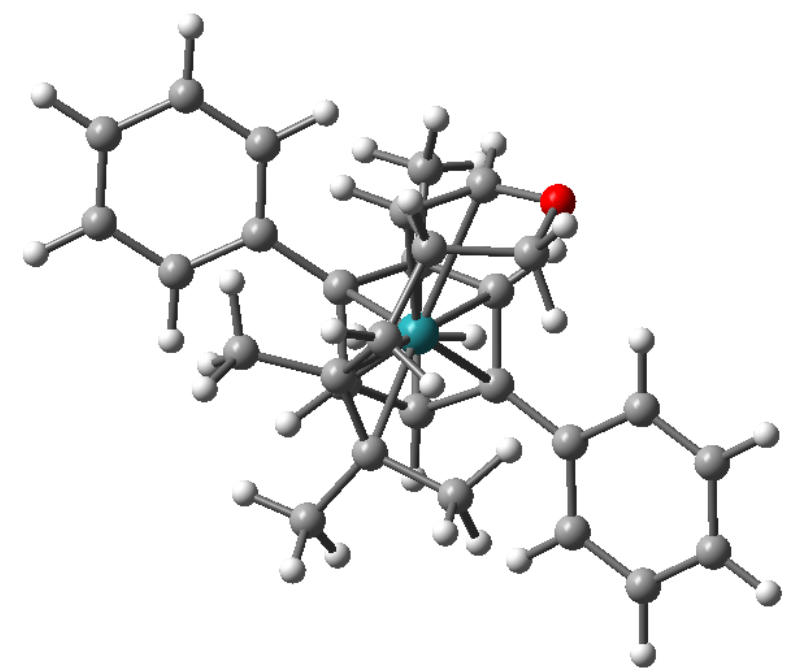

S- 101 
Path $(R)-9$ af

Int A ((R)-9af): $0.00 \mathrm{kcal}^{\mathrm{mol}^{-1}}$

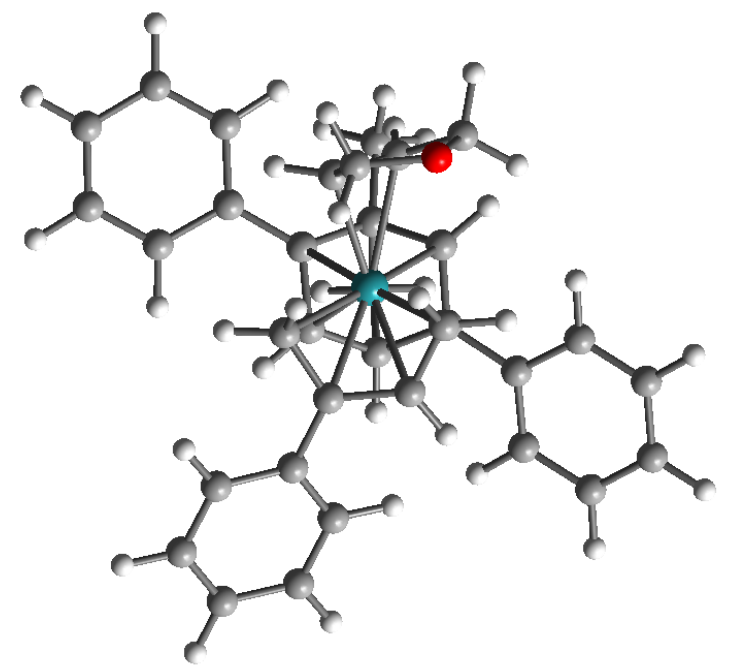

TS1 ((R)-9af): $16.23 \mathrm{kcal} \mathrm{mol}^{-1}$

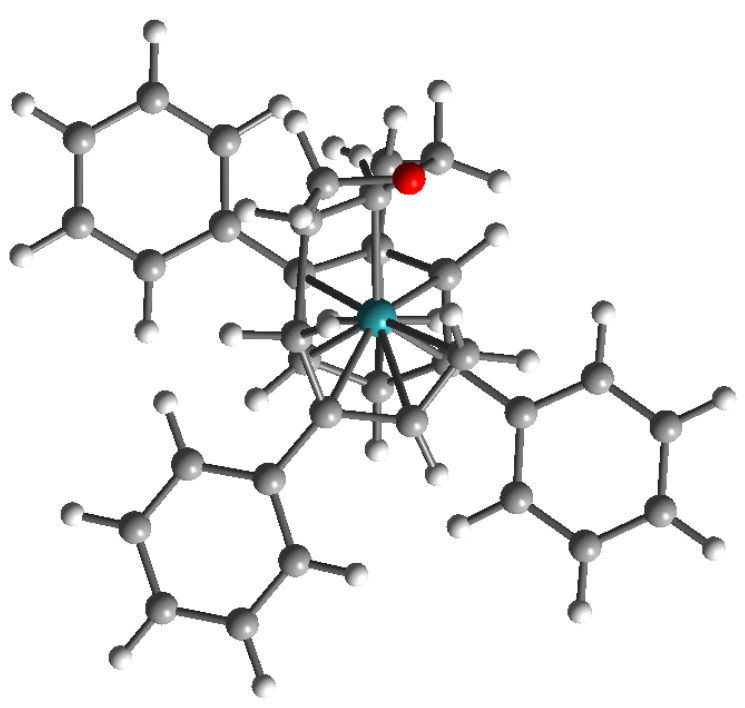

Int B ((R)-9af): $3.06 \mathrm{kcal} \mathrm{mol}^{-1}$

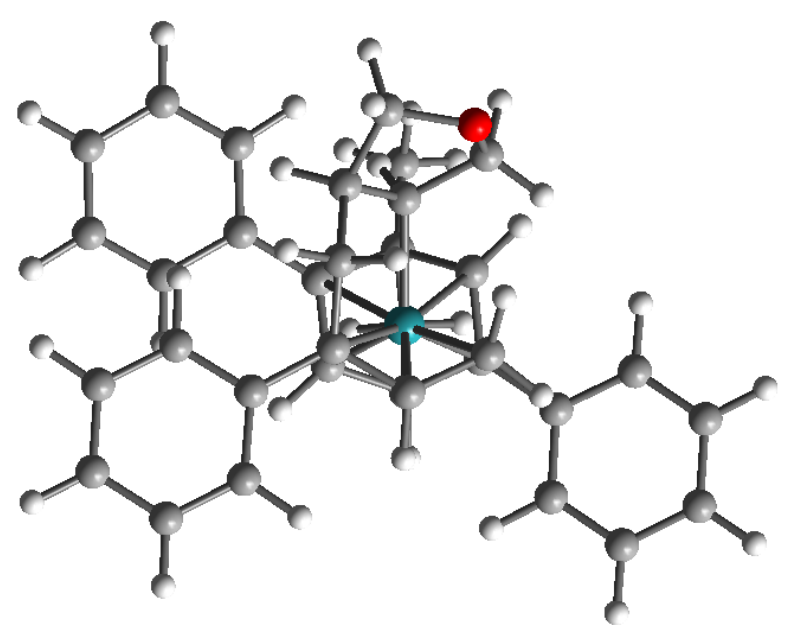

S- 102 
Int B' ((R)-9af): $3.56 \mathrm{kcal} \mathrm{mol}^{-1}$

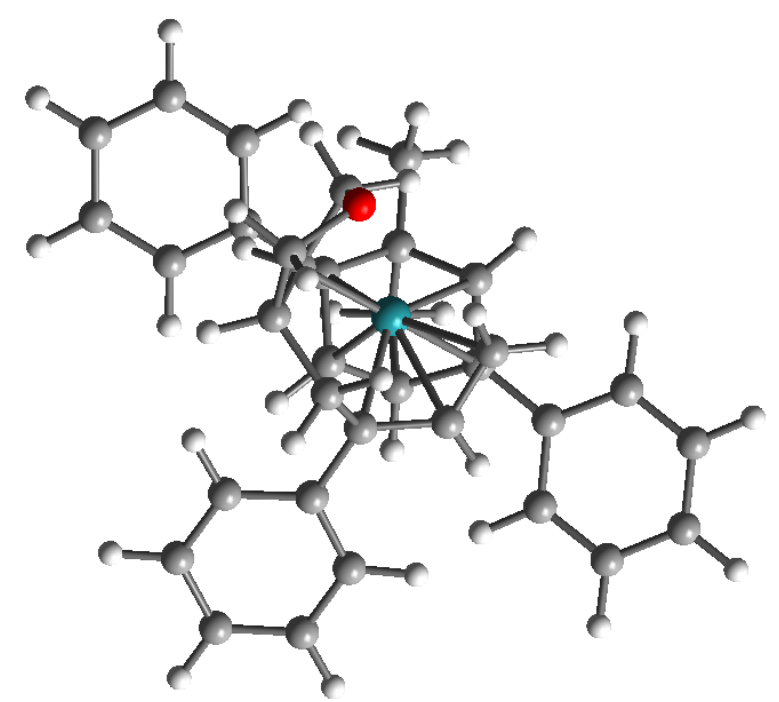

TS2 ((R)-9af): $9.61 \mathrm{kcal} \mathrm{mol}^{-1}$

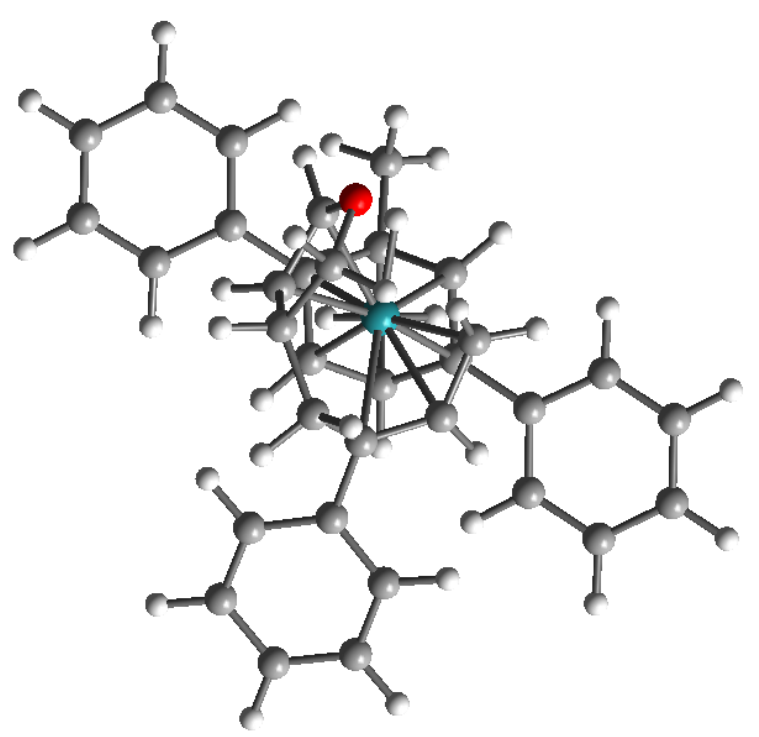

Int C ((R)-9af): $4.30 \mathrm{kcal} \mathrm{mol}^{-1}$

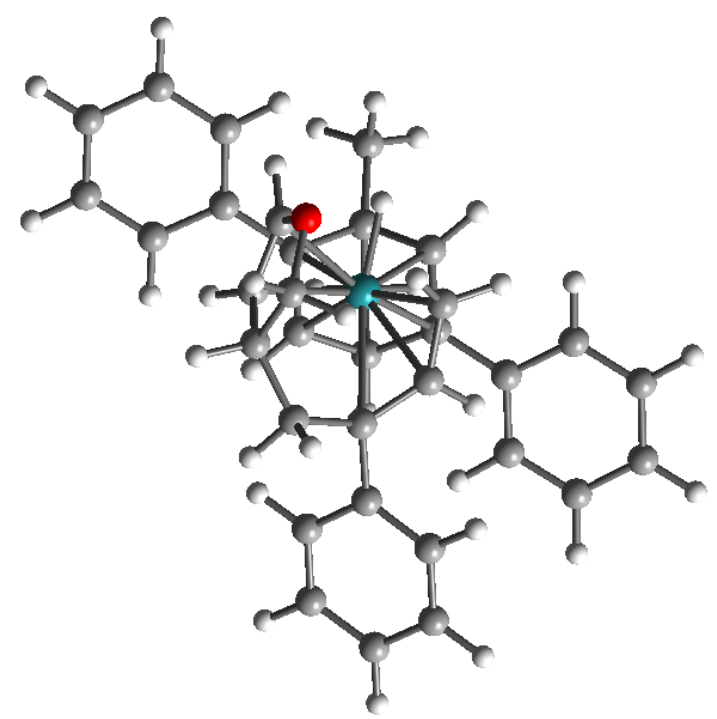

S- 103 
TS3 ((R)-9af): $14.17 \mathrm{kcal} \mathrm{mol}^{-1}$

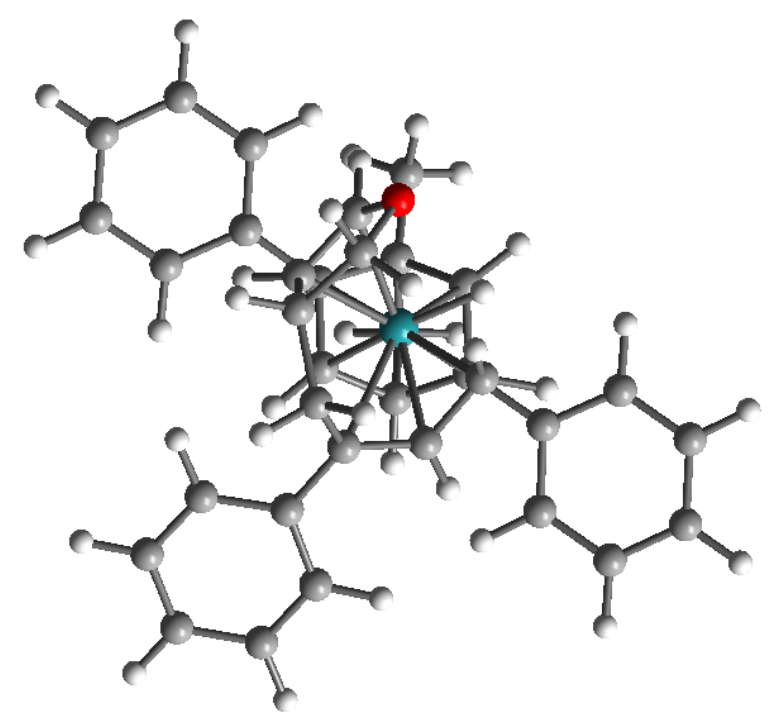

Int D ((R)-9af): -4.24 kcal mol ${ }^{-1}$

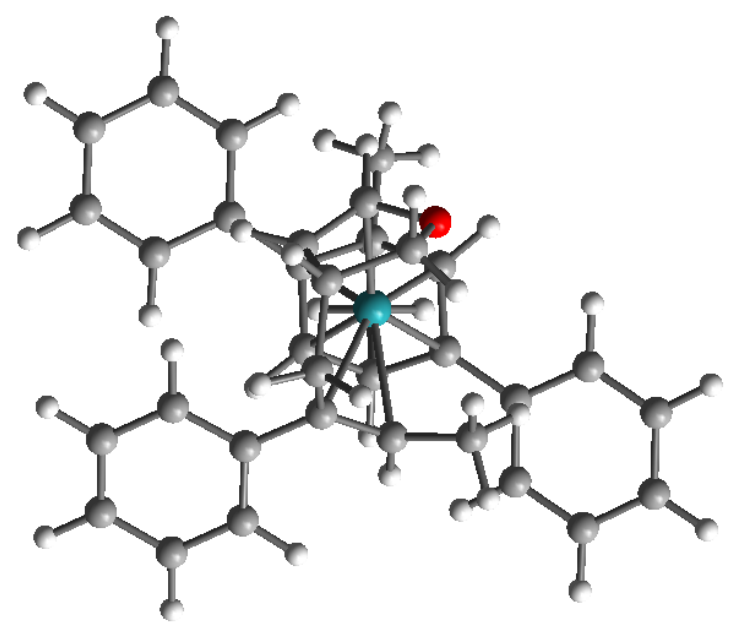




\section{Path $(S)$-9af}

Int A ((S)-9af): $2.50 \mathrm{kcal} \mathrm{mol}^{-1}$

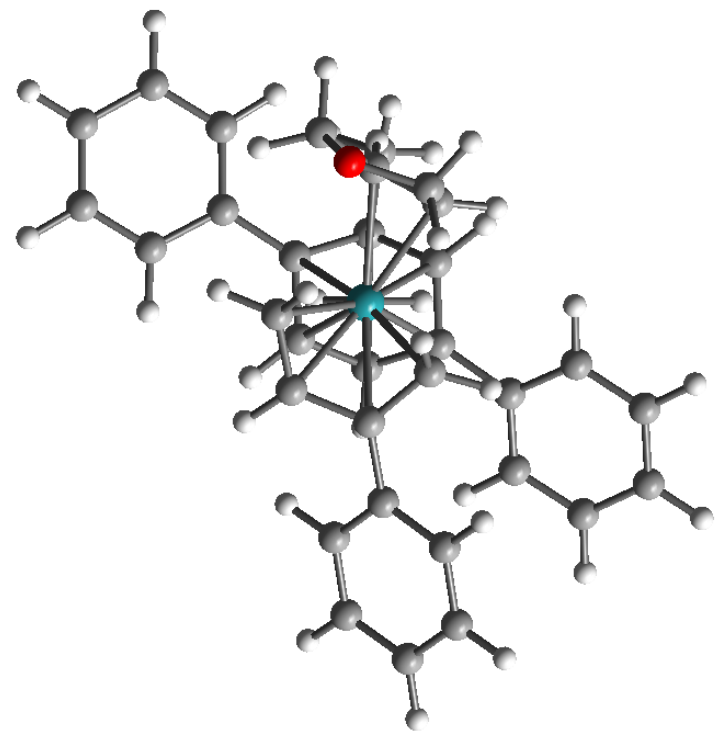

TS1 ((S)-9af): $19.38 \mathrm{kcal} \mathrm{mol}^{-1}$

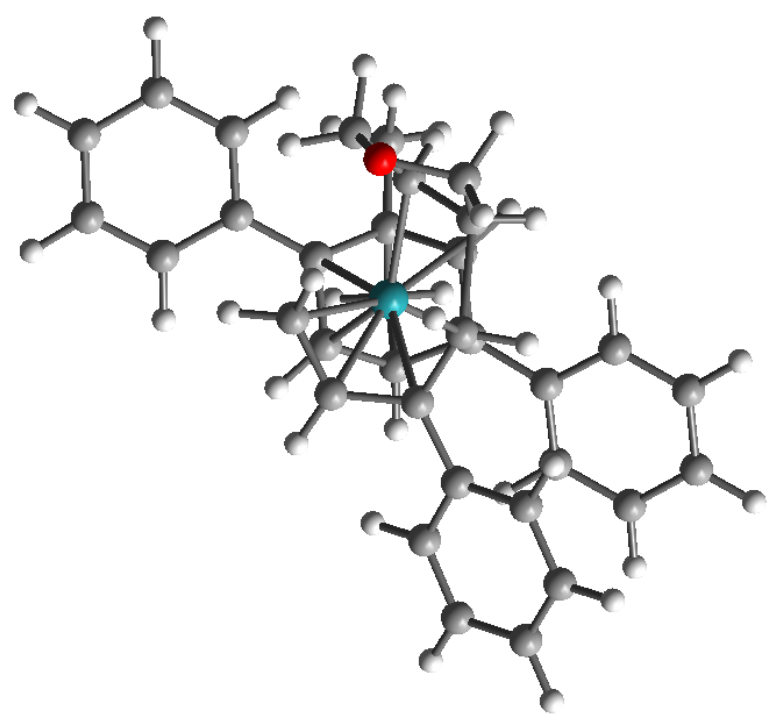

Int B ((S)-9af): $8.17 \mathrm{kcal} \mathrm{mol}^{-1}$

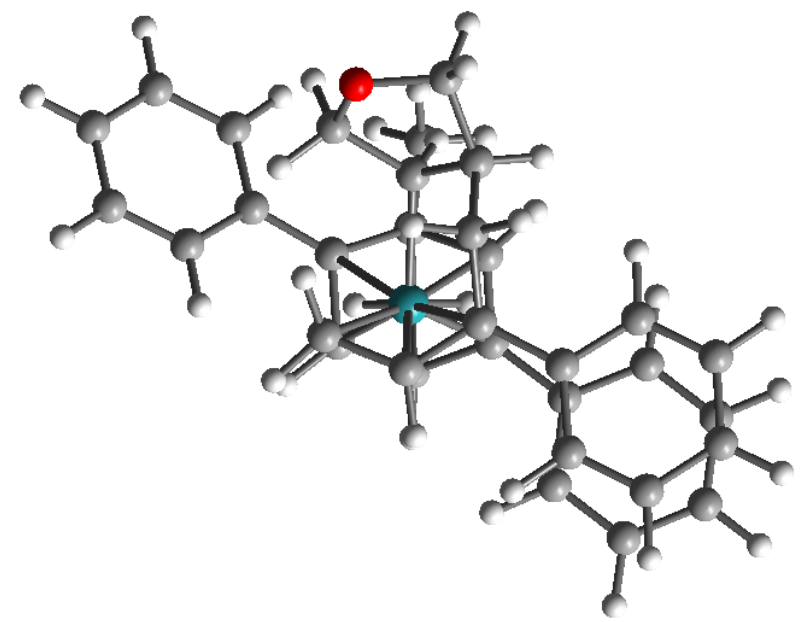

S- 105 
Int B' ((S)-9af): $4.27 \mathrm{kcal} \mathrm{mol}^{-1}$

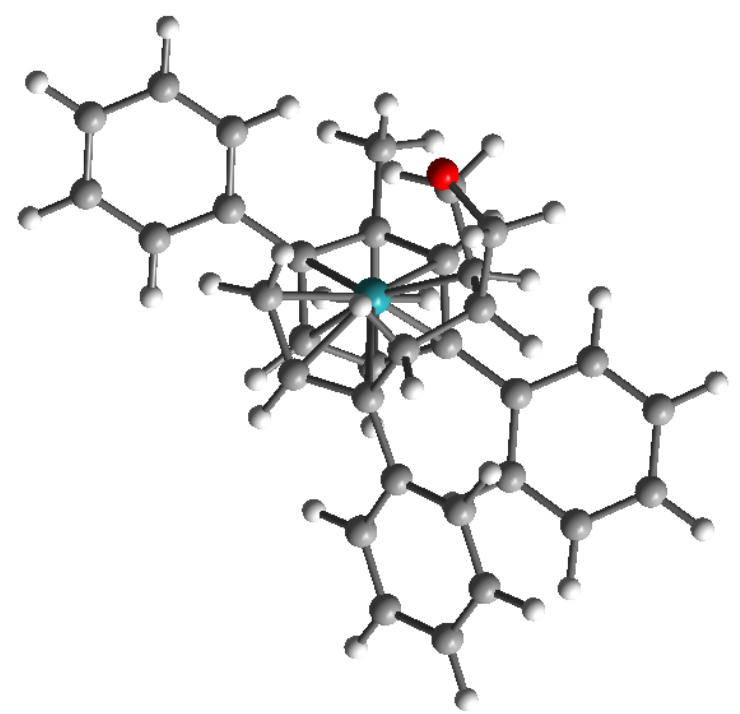

TS2 ((S)-9af): $9.13 \mathrm{kcal} \mathrm{mol}^{-1}$

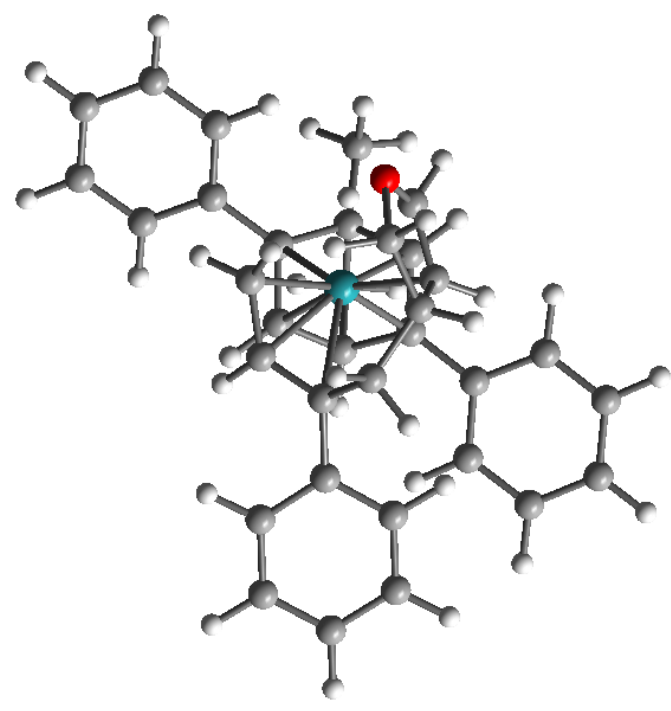

Int C ((S)-9af): $2.44 \mathrm{kcal} \mathrm{mol}^{-1}$

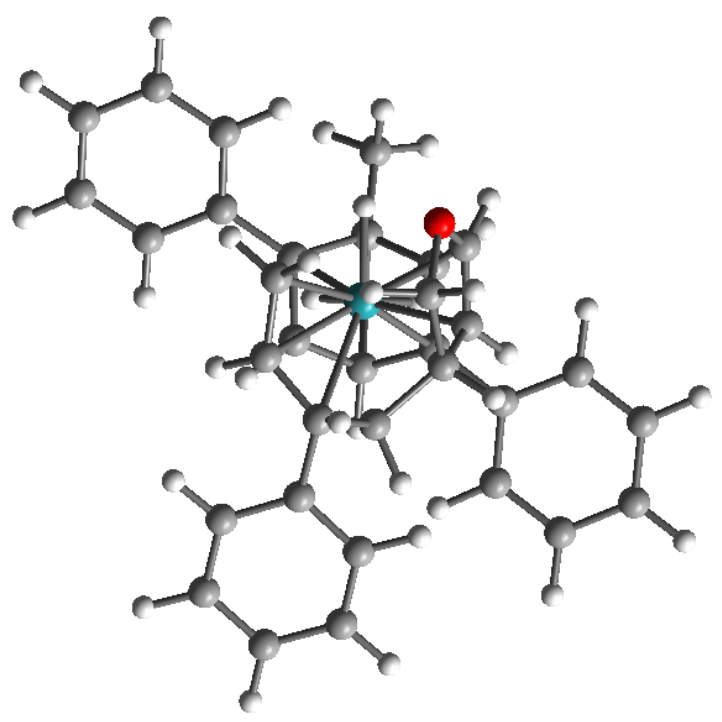

S- 106 
TS3 ((S)-9af): $9.61 \mathrm{kcal} \mathrm{mol}^{-1}$

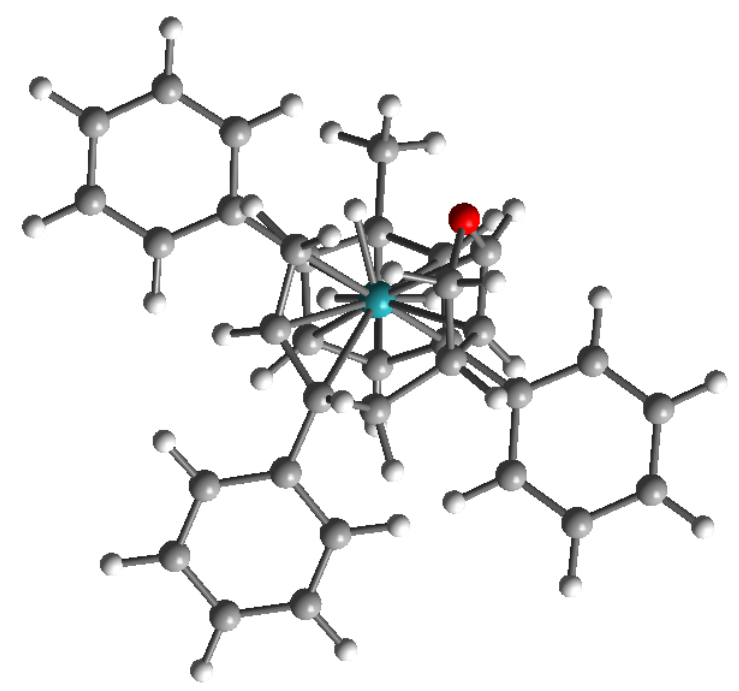

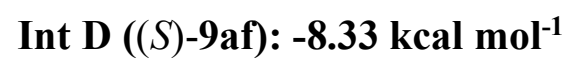

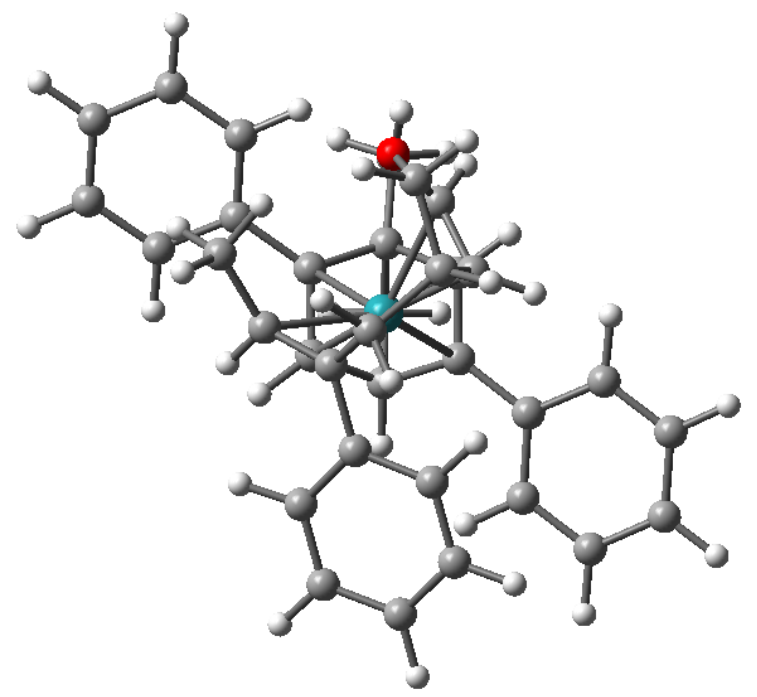


Path $(S)$-6af

Int A ((S)-6af): $1.11 \mathrm{kcal} \mathrm{mol}^{-1}$

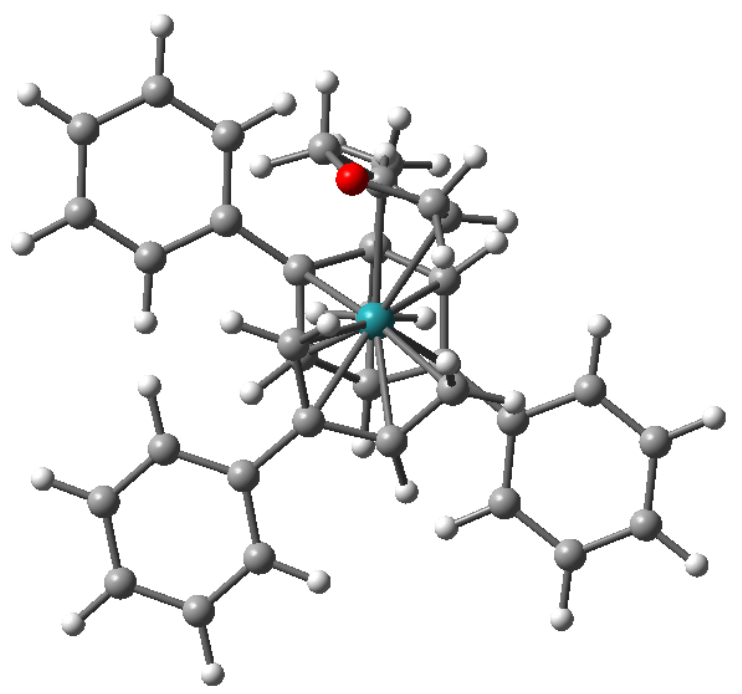

TS1 ((S)-6af): $14.87 \mathrm{kcal} \mathrm{mol}^{-1}$

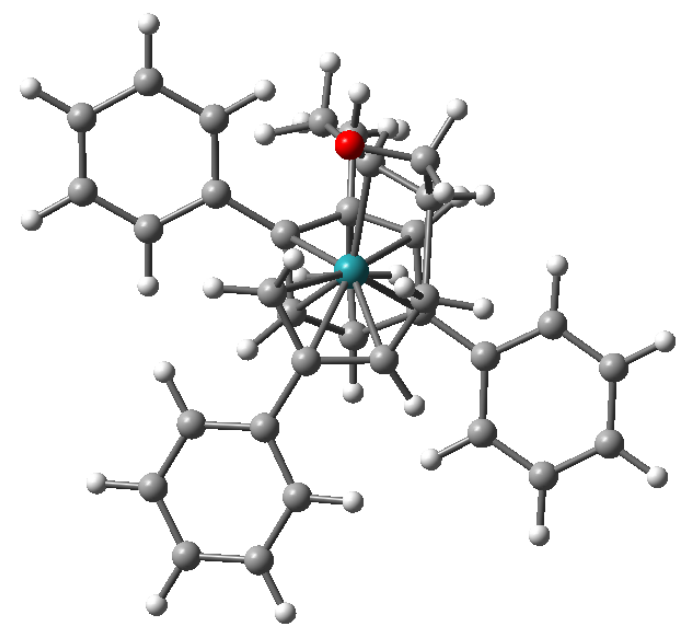

Int B ((S)-6af): $4.32 \mathrm{kcal} \mathrm{mol}^{-1}$

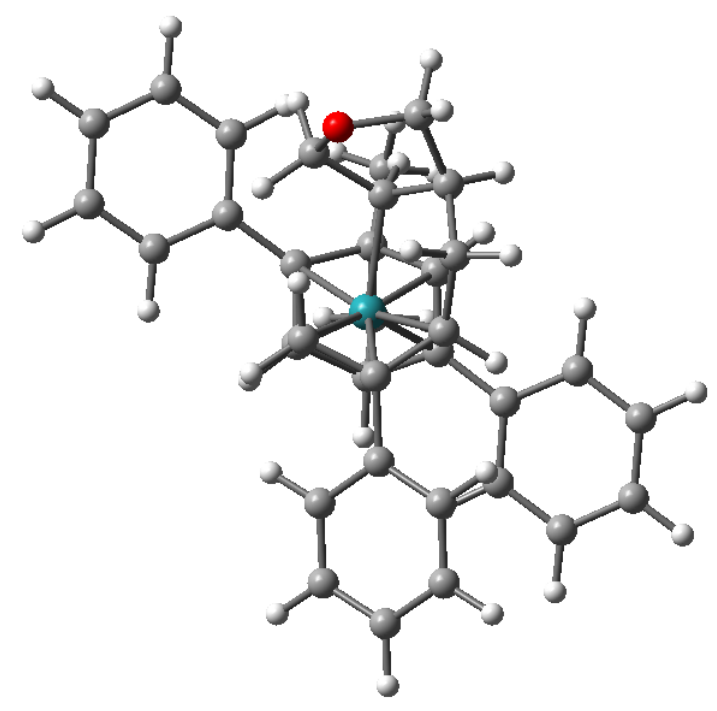

S- 108 
Int B' ((S)-6af): $-0.44 \mathrm{kcal} \mathrm{mol}^{-1}$

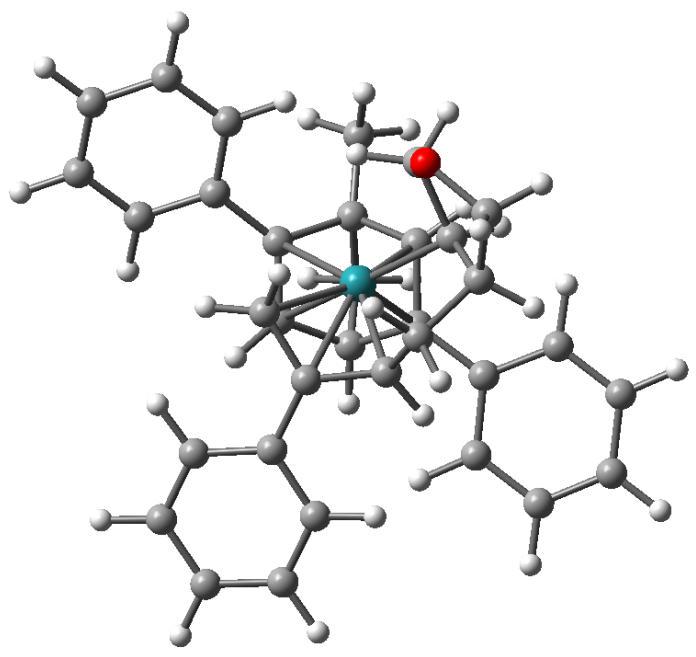

TS2 ((S)-6af): $9.38 \mathrm{kcal} \mathrm{mol}^{-1}$

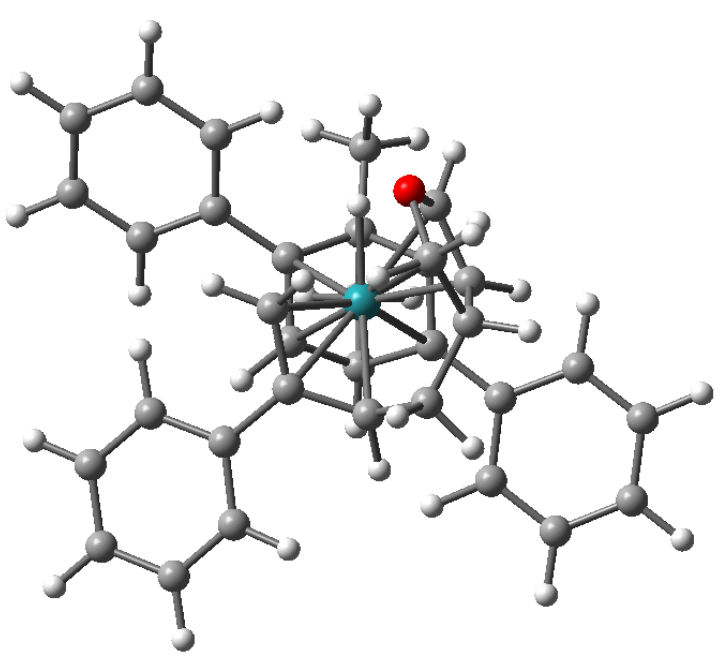

Int C ((S)-6af): $4.01 \mathrm{kcal} \mathrm{mol}^{-1}$

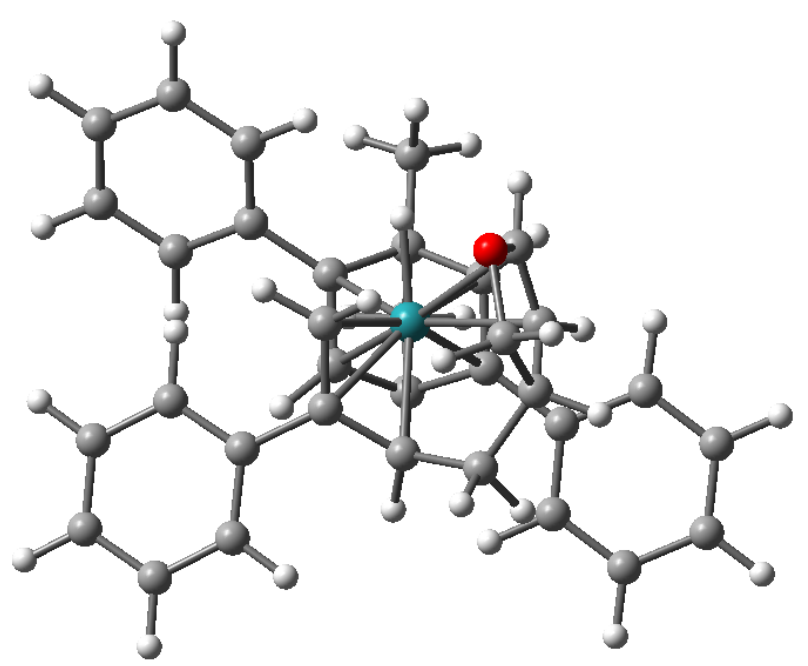

S- 109 
TS3 ((S)-6af): $14.17 \mathrm{kcal} \mathrm{mol}^{-1}$

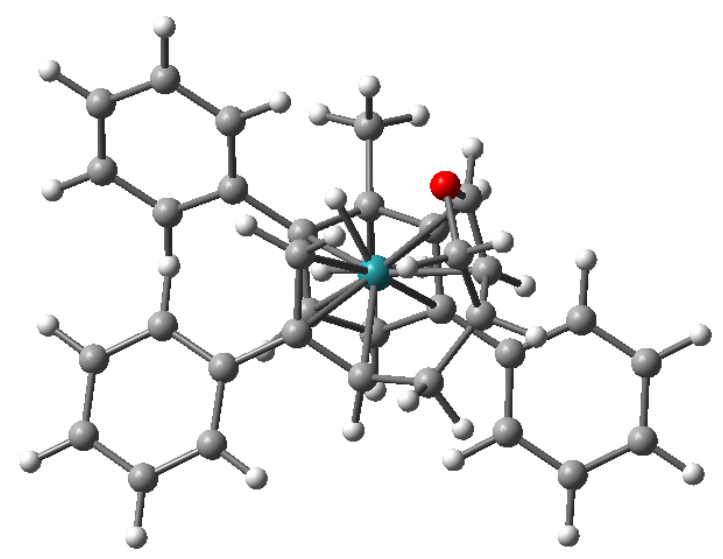

Int D ((S)-6af): -7.96 kcal mol ${ }^{-1}$

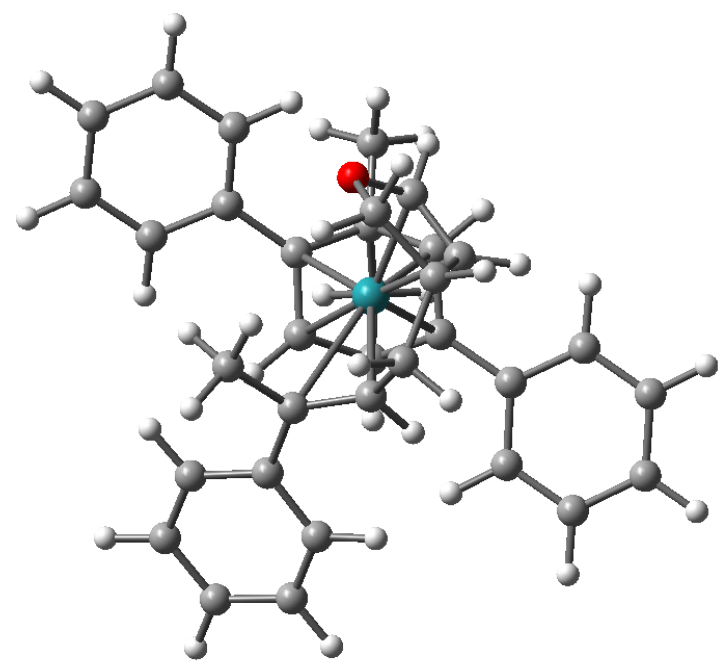


Path $(R)$-6af

Int A ((R)-6af): $4.48 \mathrm{kcal} \mathrm{mol}^{-1}$

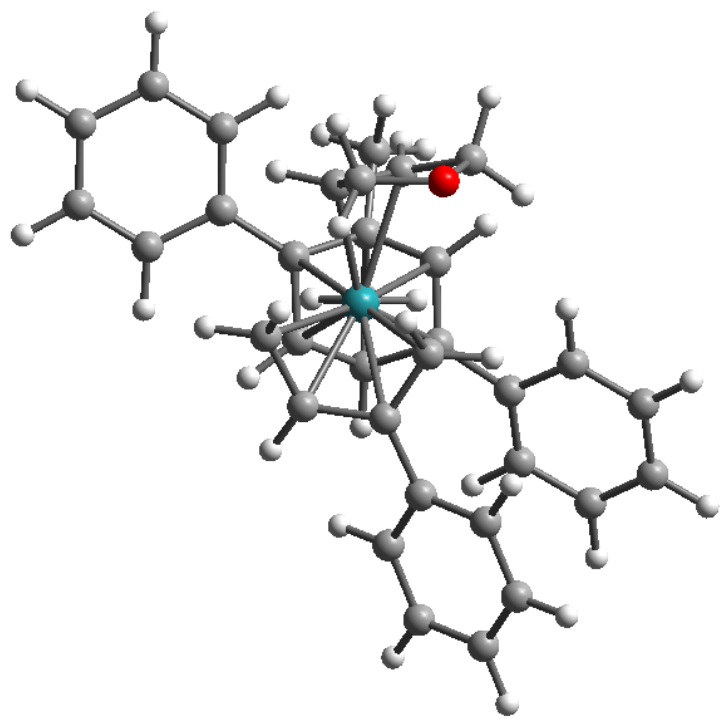

TS1 ((R)-6af): $18.95 \mathrm{kcal} \mathrm{mol}^{-1}$

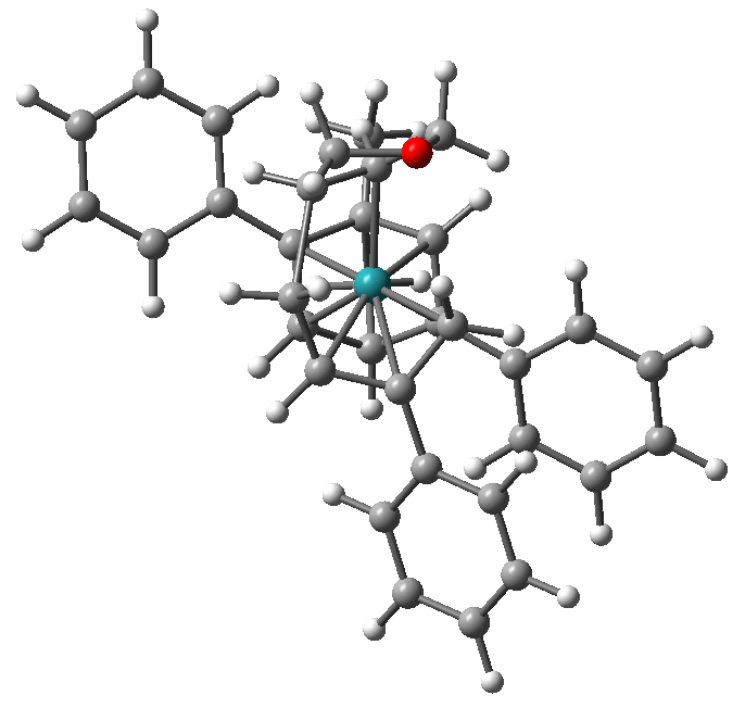

Int B ((R)-6af): $3.23 \mathrm{kcal} \mathrm{mol}^{-1}$

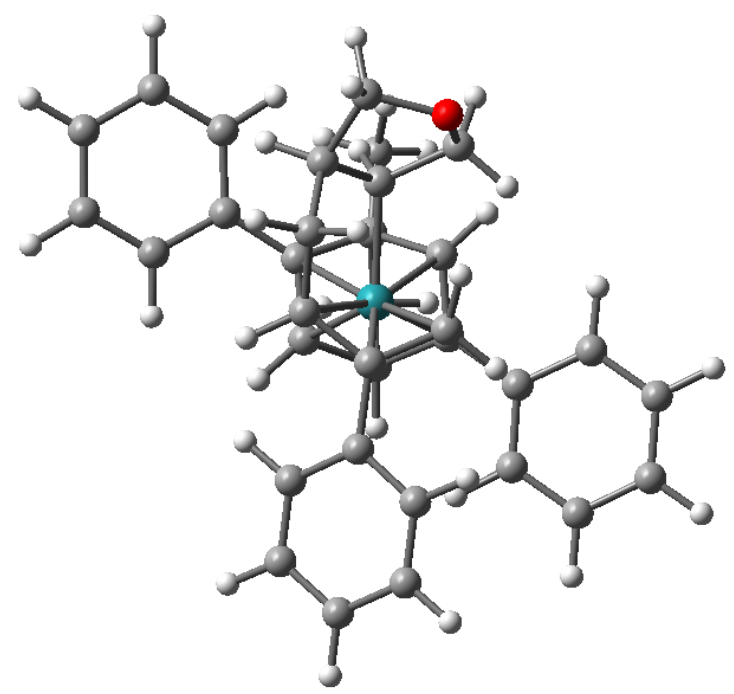

S- 111 
Int B' ((R)-6af): $0.32 \mathrm{kcal} \mathrm{mol}^{-1}$

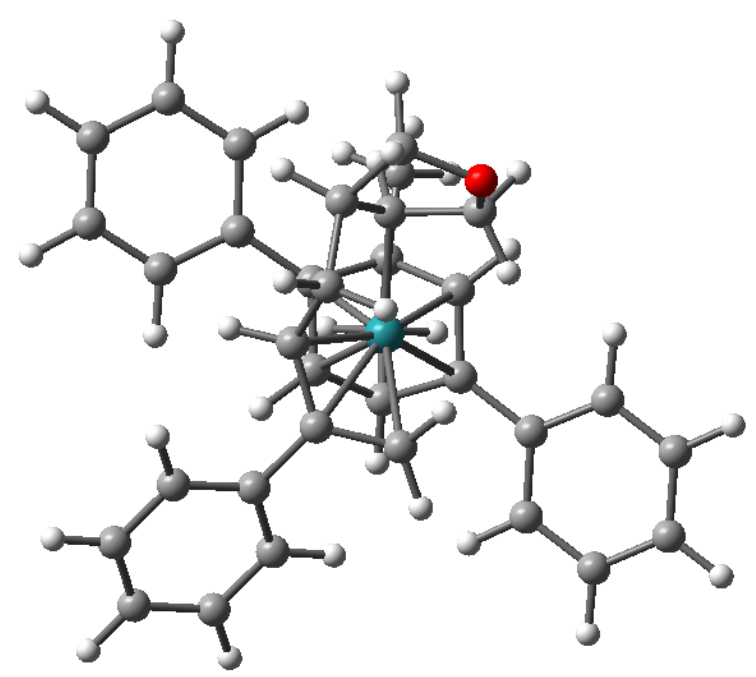

TS2 ((R)-6af): $14.82 \mathrm{kcal} \mathrm{mol}^{-1}$

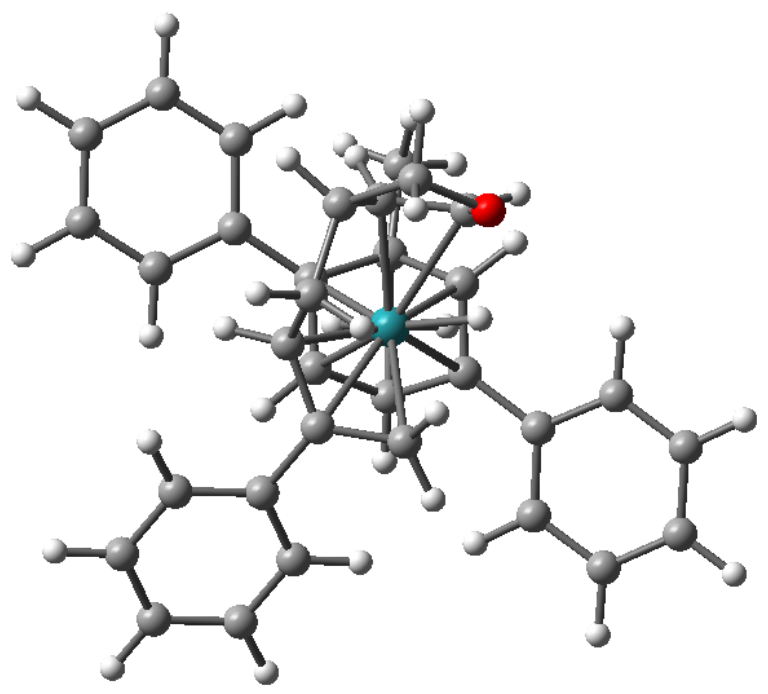

Int C ((R)-6af): $10.81 \mathrm{kcal} \mathrm{mol}^{-1}$

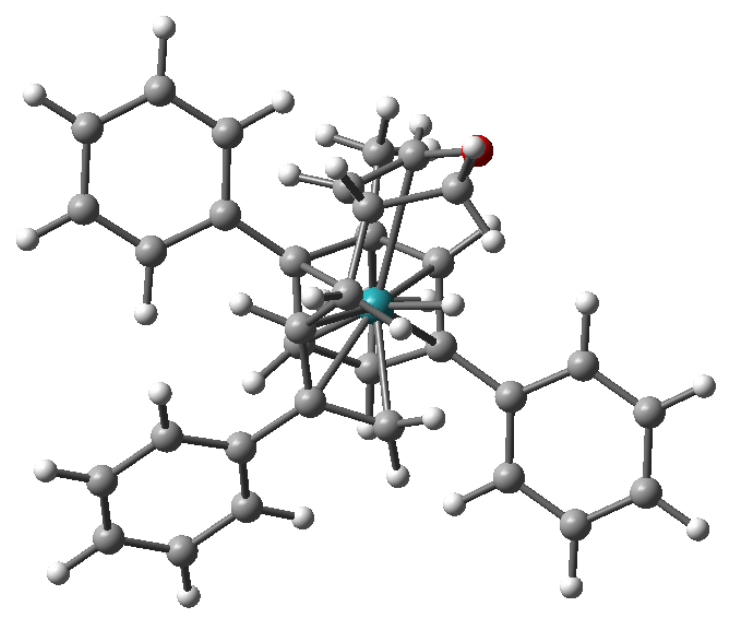

S- 112 
TS3 ((R)-6af): $12.44 \mathrm{kcal} \mathrm{mol}^{-1}$

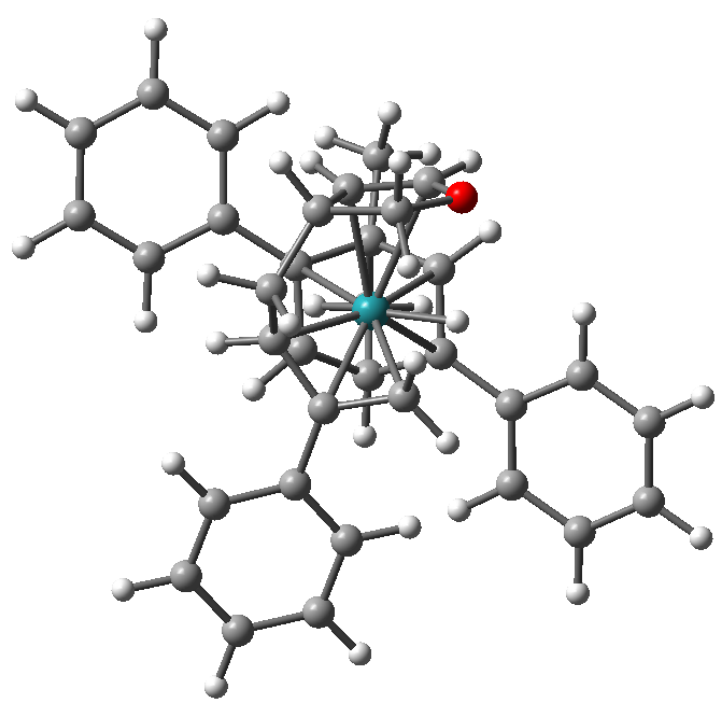

Int D ((R)-6af): -4.04 kcal mol-1

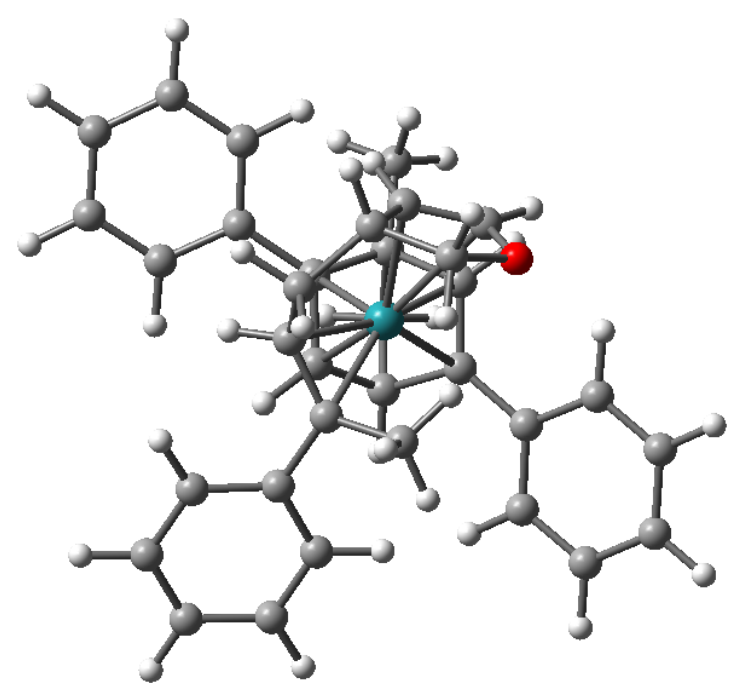

